\title{
Floating Breakwater \\ Theoretical study of a dynamic wave attenuating system
}

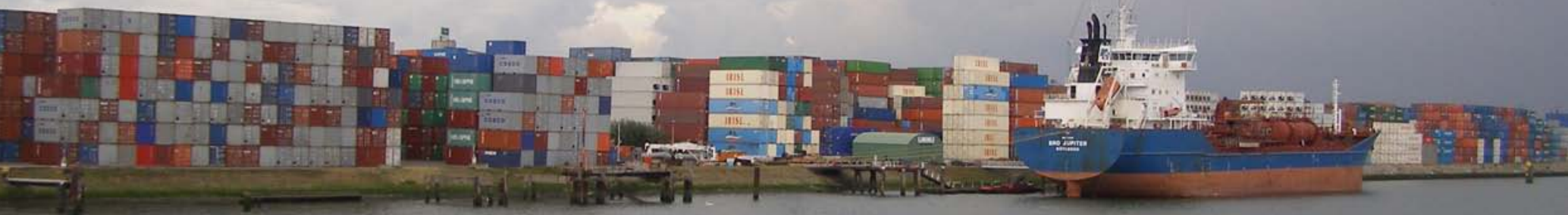

M.W.Fousert

December $15^{\text {th }} 2006$ 



\section{COLOPHON}

\section{Floating Breakwater}

A Theoretical study of a dynamic wave attenuating system

Final report of the master thesis

Delft, 2006

Delft University of Technology

Faculty of Civil Engineering and Geosciences

Section of Hydraulic Engineering

\section{COMMITTEE MASTER THESIS}

Prof.drs.ir. J.K.Vrijling Chairman Committee, TU Delft section of hydraulic engineering

ir. W.F.Molenaar

Daily supervisor

TU Delft section of hydraulic engineering

ir. J.L.F. van Kessel

TU Delft Maritime engineering

\section{GRADUATE STUDENT}

M.W.Fousert B.Sc.

Menno ter Braaklaan 203

2624 TE Delft

Tel. 06-41513755

Student number: 1091611

\section{DELFT UNIVERSITY OF TECHNOLOGY}

Section of hydraulic engineering

PO box 5048

2600 GA Delft, the Netherlands

Front cover: Rotterdam Eemhaven container terminal with an artist impression of a floating breakwater. 


\section{$A B S T R A C T$}

Ever since progressive engineers came up with the idea of creating floating structures in the sea, many studies and models tests were performed in order to develop the optimal floating breakwater.

Although the advantages of a floating structure seem to be rather obvious, the use of these structures is limited, mainly due to the complex hydrodynamic behaviour, the reliability and the costs of these structures.

International container shipping is one of the most dynamic economic sectors of the past years. Higher efficiency of the loading and unloading processes and the increase in size of container vessels contributed to the development of the Floating Transhipment Container Terminal (FTCT). The mutual motions of vessel and FTCT may not exceed a certain maximum to guarantee optimal harbour efficiency. A transportable, reusable floating breakwater is necessary to increase the efficiency of a FTCT.

The objective of this thesis was to analyse the hydrodynamic behaviour of the floating breakwater and to improve the performance of the floating breakwater. A model, the so-called Rectangular Floating Breakwater Design Model (ReFBreak-Model), had to be developed that served as a general design tool to determine the dimensions of the floating breakwater.

First of all, the calculation parameters, requirements and boundary conditions had to be determined. The harbour efficiency puts demands on the performance of the floating breakwater and is an important factor to determine whether the floating breakwater concept is successful or not. The hydrodynamic coefficients for rectangular floating bodies, measured by Vugts (1970) are used in the calculations and were extrapolated to a wider wave frequency range. The floating breakwater itself has a rectangular shape with the possibility of placing a rigid screen underneath it.

Two-dimensional calculations were performed to determine the influence of several structural variables on the hydrodynamic behaviour of the floating breakwater. The calculations were performed in regular, sinus-shaped beam-on waves in order to be able to determine the floating breakwater behaviour in different wave periods.

The hydrodynamic behaviour of floating breakwaters is influenced by many factors. However, only a limited number could be analyzed in the underlying thesis work. Therefore, a rectangular floating body with varying width, draft and mass has been assumed as the basic shape of the floating breakwater. Other structural variables are the mooring line damping- and stiffness and the possibility of placing a rigid screen underneath the floating structure.

Hydrodynamic behaviour as calculated by the ReFBreak-model has been checked with the three dimensional potential-theory computer model DELFRAC to determine the reliability of the calculations. Wave transmission to the harbour side of the floating breakwater has been calculated for several floating breakwater layouts.

The theoretical study proves that a floating breakwater is able to attenuate waves when the structural layout is optimal.

- $\quad$ The floating breakwater is able to attenuate regular beam waves with periods up to 17 seconds.

- $\quad$ This kind of floating breakwater must be able to adapt its structural appearance on the prevalent wave periods.

- The draft-width relation is the most important factor that determines the performance of the floating breakwater.

Since the results of this model are based on pure theoretical calculations, further research is necessary to investigate the influence of irregular, oblique (Ocean) waves on the performance of the adaptable floating breakwater. 


\section{PREFACE}

This research has been performed as a final thesis of the M.sc. program at the faculty of Civil Engineering and Geosciences, Delft University of Technology. The research is performed under the authority of the section of hydraulic engineering and was carried out from January 2006 until November 2006.

The results are presented in this report and will be a basis for further research on this topic. They can be used as an initial design tool to determine the dimensions and the important factors in the development of full-scale floating breakwaters.

\section{ACKNOWLEDGEMENTS}

I would like to thank the following people for their contributions to this thesis:

First of all my appreciation and thanks go to the people of my graduation committee, J.K.Vrijling, W.Molenaar and J.van Kessel for their advise and help during the whole research.

I'm very grateful to my supervisor at Maritime Engineering, Jan van Kessel for his patient and useful help during the calculation process.

Finally, I would like to thank all friends and family for their support and interest throughout the years. 


\section{TABLE OF CONTENTS}

COLOPHON

ABSTRACT

PREF A CE

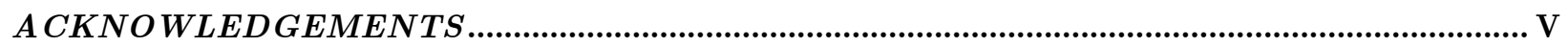

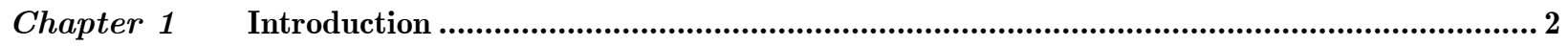

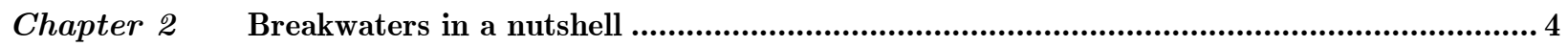

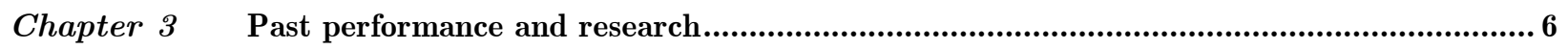

$3.1 \quad$ Why floating breakwaters? …

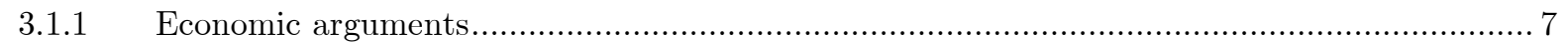

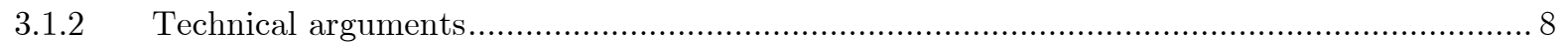

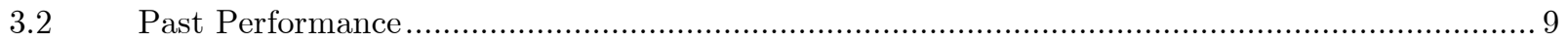

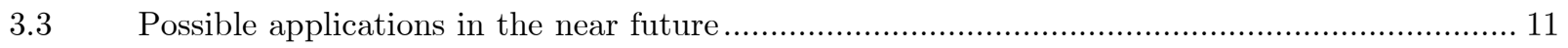

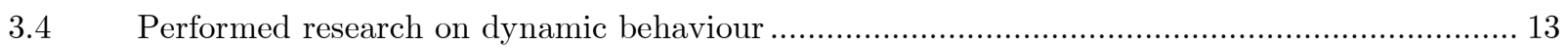

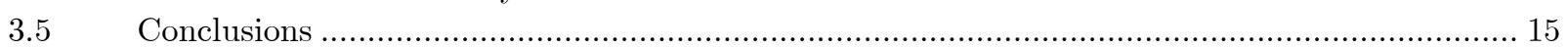

Chapter $4 \quad$ Problem definition \& Objectives.......................................................................................... 16

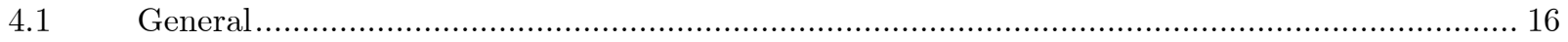

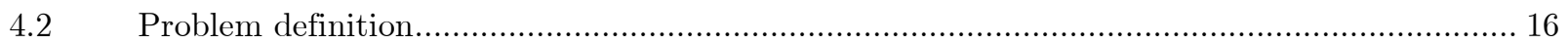

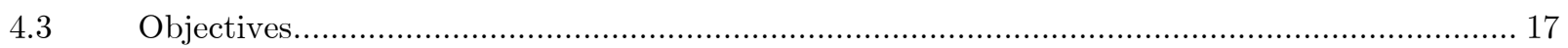

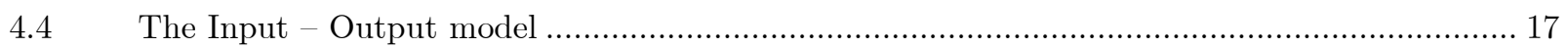

Chapter $5 \quad$ Boundary conditions \& Requirements ............................................................................ 20

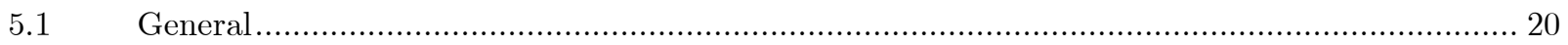

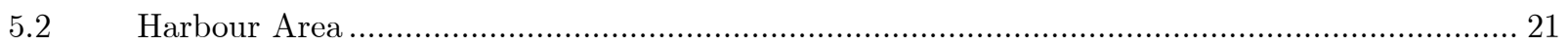

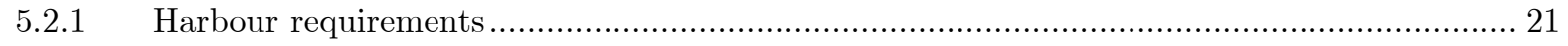

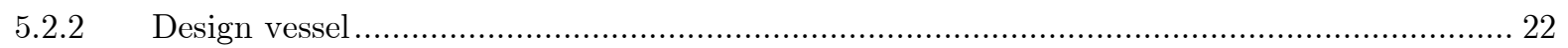

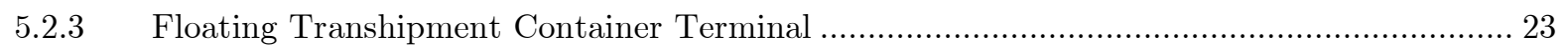

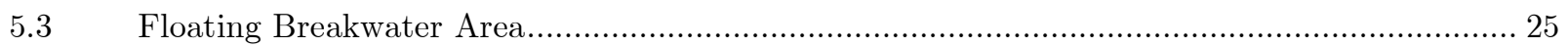

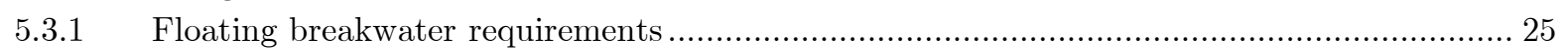

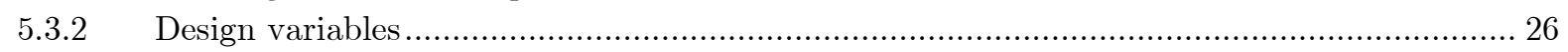

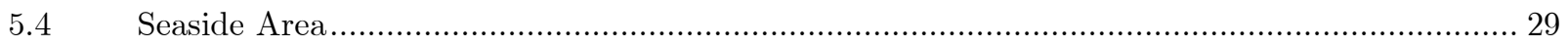

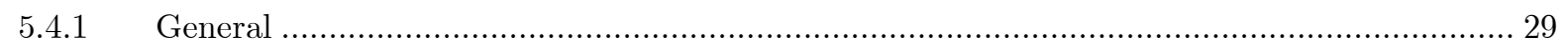

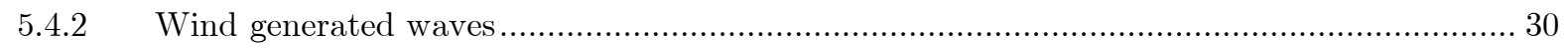

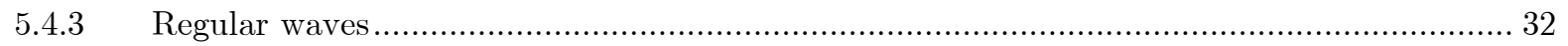

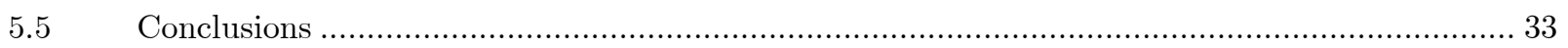

Chapter $6 \quad$ Theoretical Rectangular Floating Breakwater Model ReFBreak ..................................... 34

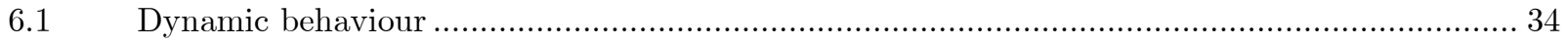

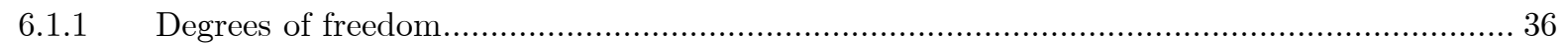

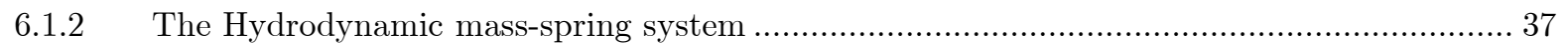

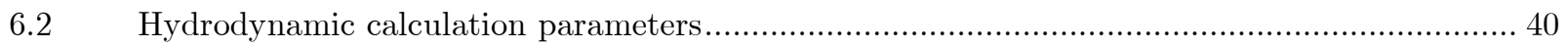

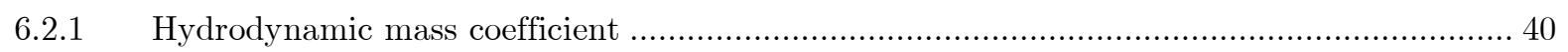

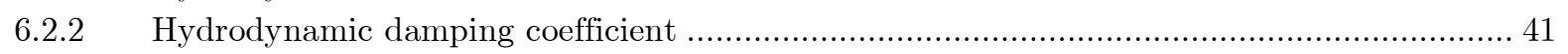

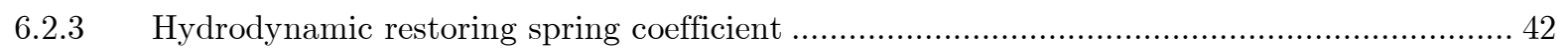

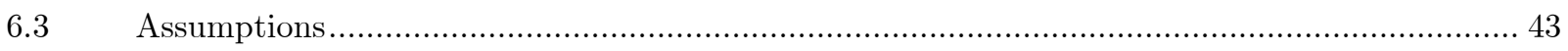




\begin{tabular}{|c|c|}
\hline & Floating Breakwater \\
\hline 6.4 & ㄱ. \\
\hline 6.4 .1 & (1) \\
\hline 6.4 .2 & Heave.... \\
\hline 6.4 .3 & 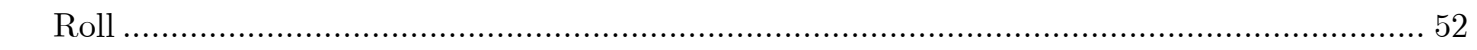 \\
\hline 6.4 .4 & 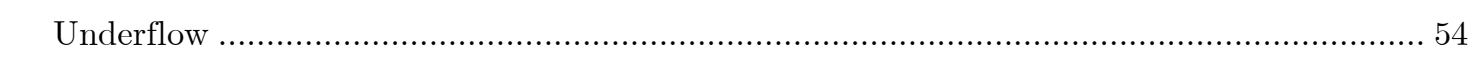 \\
\hline 6.4 .5 & 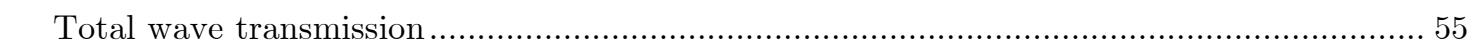 \\
\hline 6.5 & 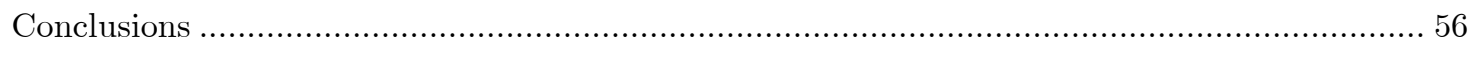 \\
\hline
\end{tabular}

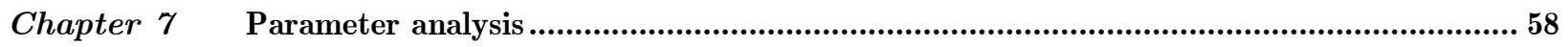

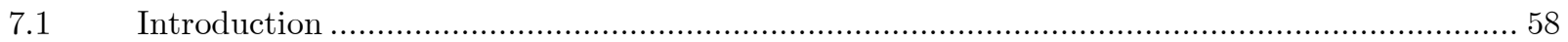

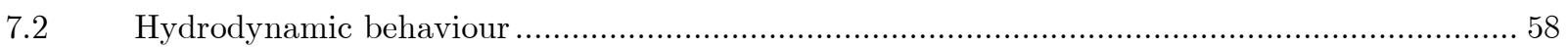

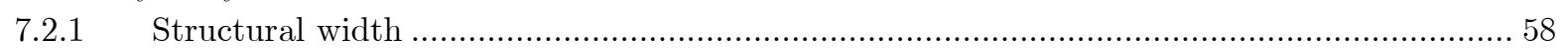

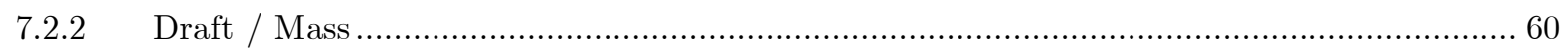

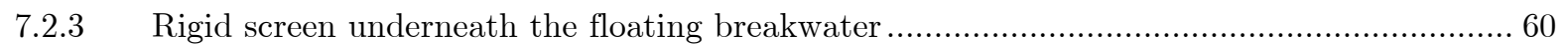

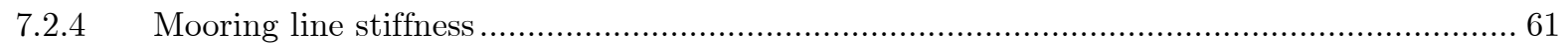

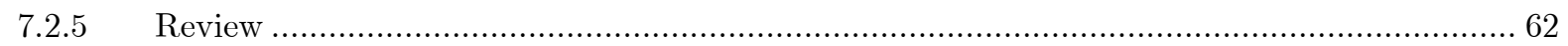

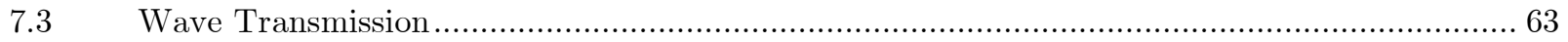

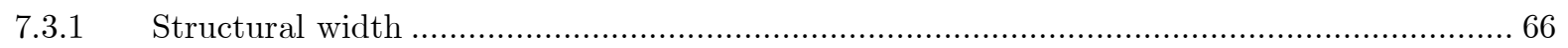

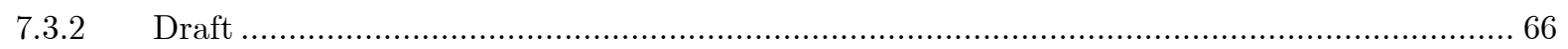

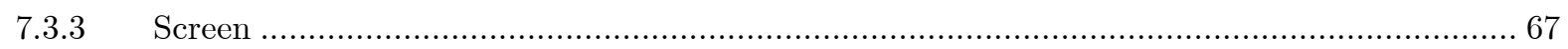

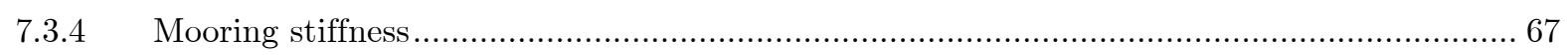

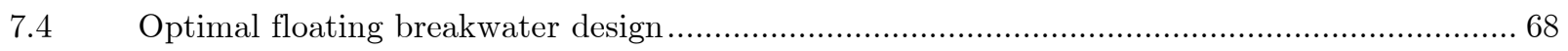

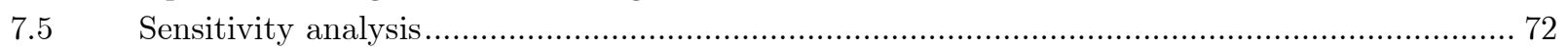

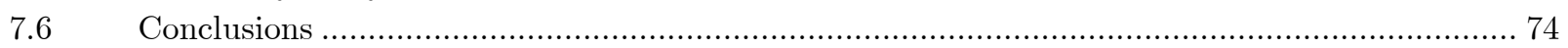

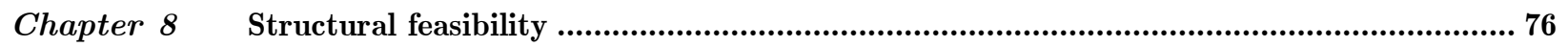

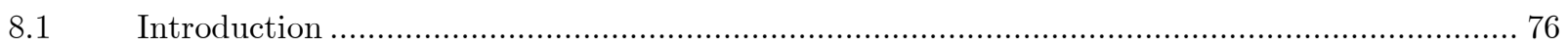

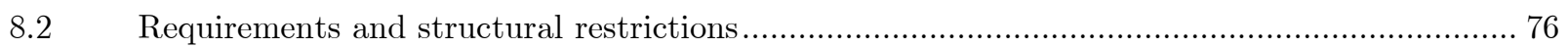

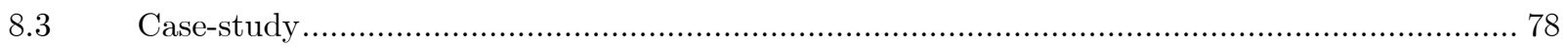

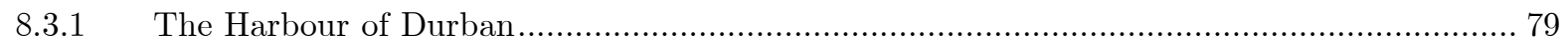

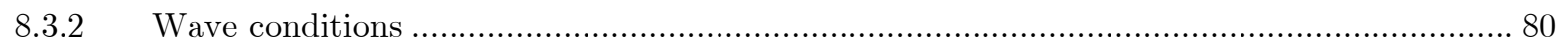

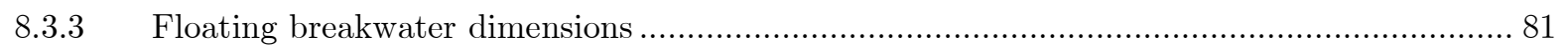

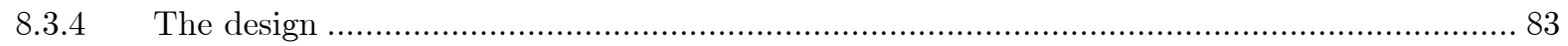

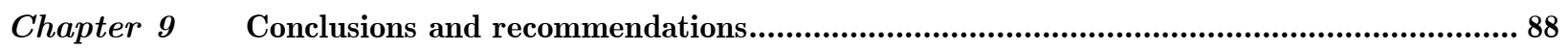

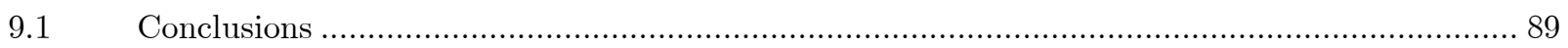

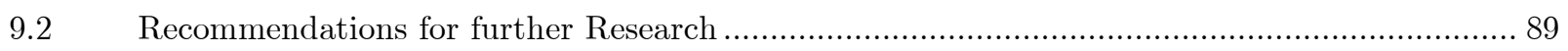




\section{LIST OF FIGURES}

Figure 2-1: Several breakwater structures. …

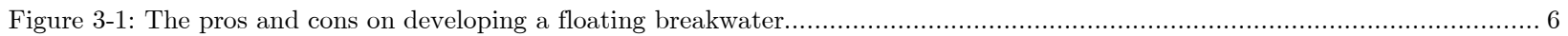

Figure 3-2: Comparison of construction cost / $\mathrm{m} 1$ depending on the water depth. ........................................................... 7

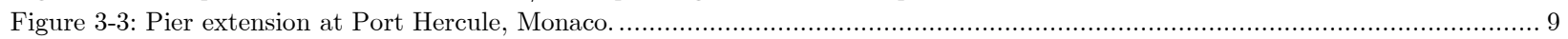

Figure 3-4: Maximum ship size by year of construction (until October 2006)

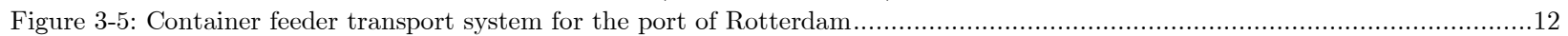

Figure 3-6: Performance of several types of structures. …

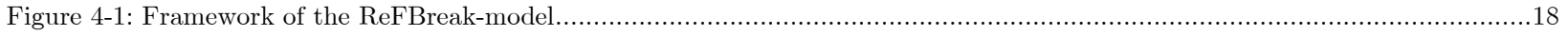

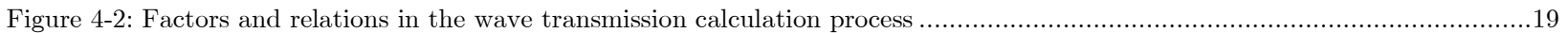

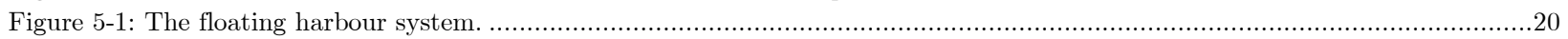

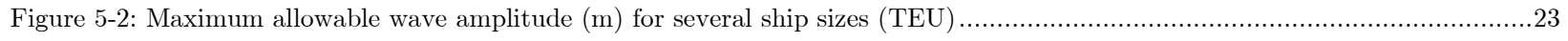

Figure 5-3: FTCT cross-section (from Ali (2005))

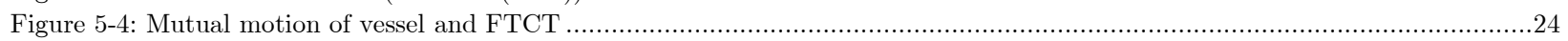

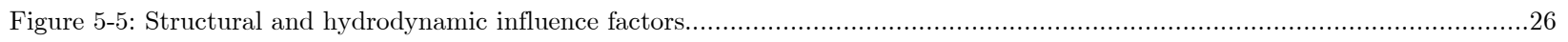

Figure 5-6: Tidal influence on the mooring line influence.

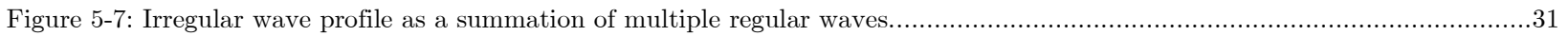

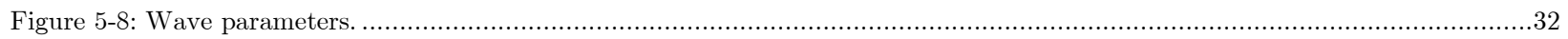

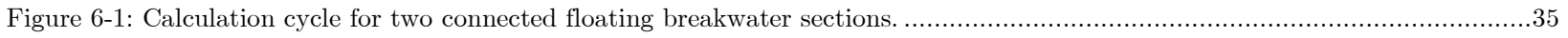

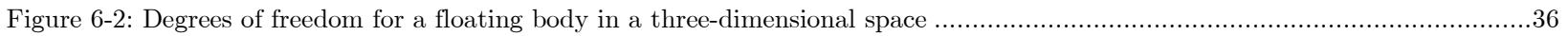

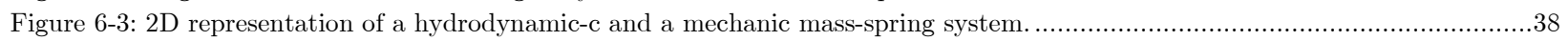

Figure 6-4: Influence of the damping, the mass and the spring stiffness on the dynamic behaviour of a mass-spring system. ............39

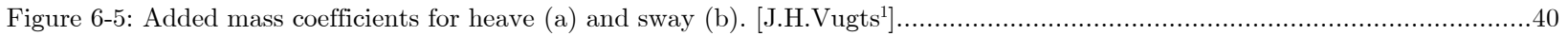

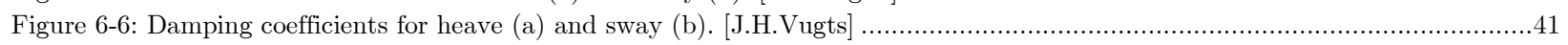

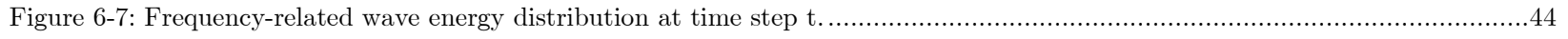

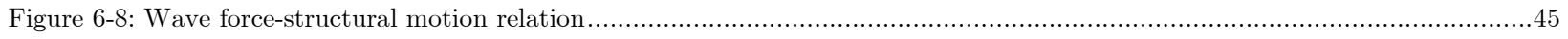

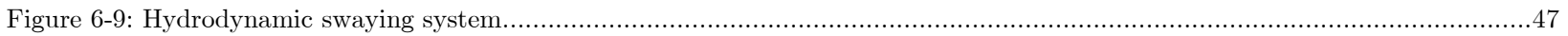

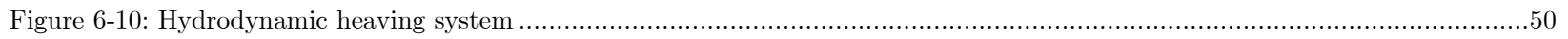

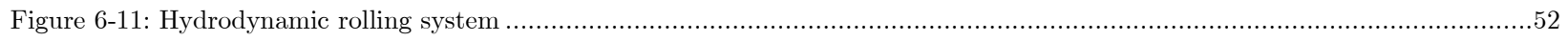

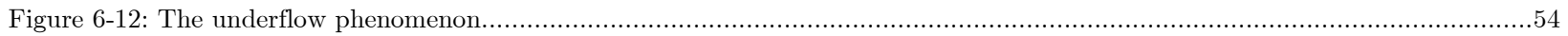

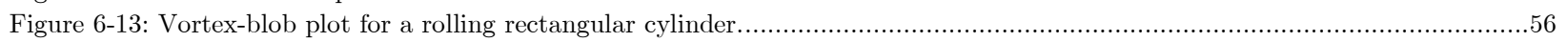

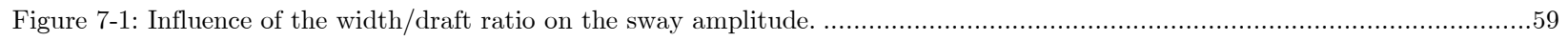

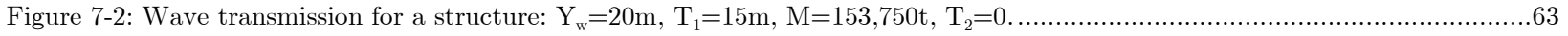

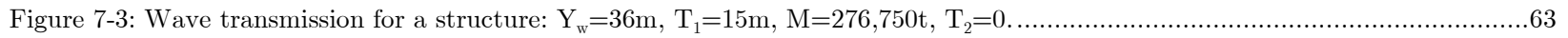

Figure 7-4: Three dimensional over view of the transmitted wave amplitude ratio (RAO) with a varying wave period and draft. ....64

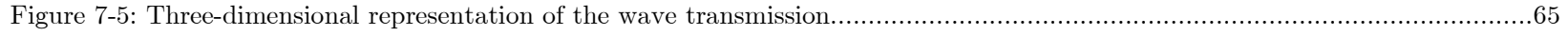

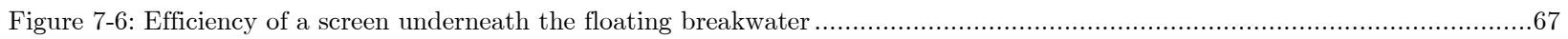

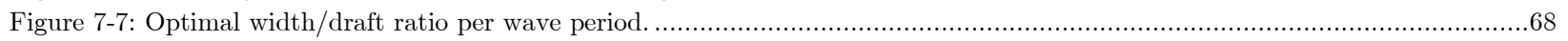

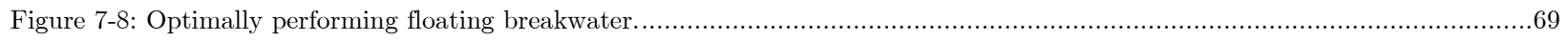

Figure 7-9: Floating breakwater performance (according to the dashed line in figure $7-8$ ) .................................................

Figure 7-10: Maximum allowed incoming wave heights for a 6000 TEU container vessel............................................................70

Figure 7-11: Heave reaction of the FTCT-feeder system and wave transmission of the floating breakwater. ................................71

Figure 7-12: Deviation of the transmission coefficient when a standard deviation of $10 \%$ is applied for the heave hydrodynamic added mass. ..........72

Figure 7-13: Deviation of the transmission coefficient when a standard deviation of $10 \%$ is applied for the heave hydrodynamic damping. ..............73

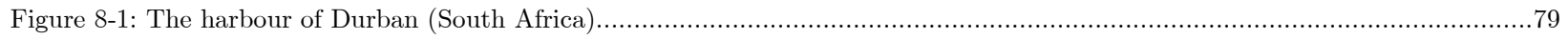

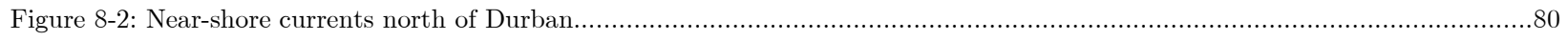

Figure 8-3: Significant wave height and probability density function per wave period in the Durban region. …….......................81

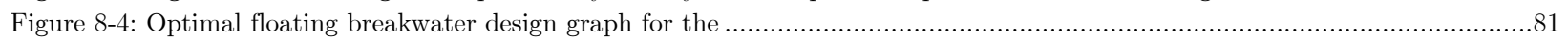

Figure 8-5: Significant wave height and maximum allowed wave height for a 6000 TEU container vessel. ................................82

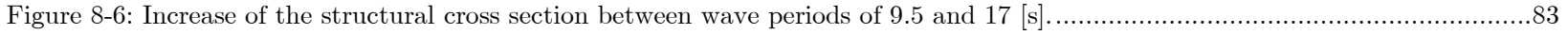

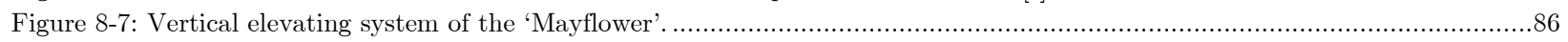

\section{LIST OF TABLES}

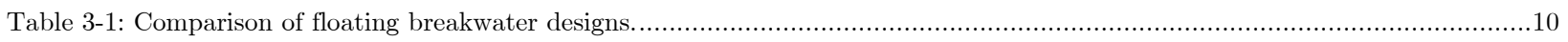

Table 5-1: Recommended ship motions during harbour operations. Motions refer to peak-peak values (except for sway: zero-peak). 21

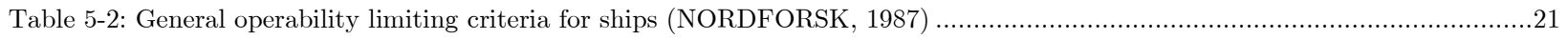

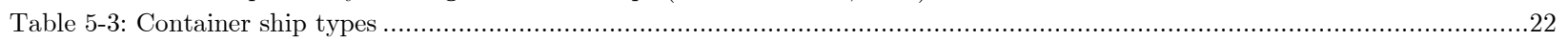

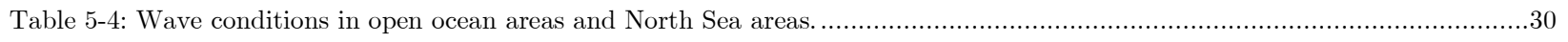

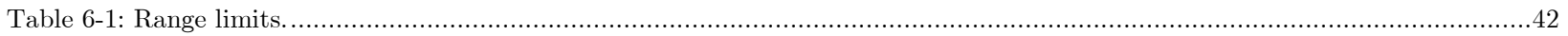

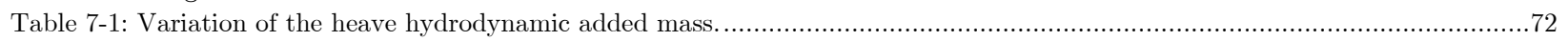

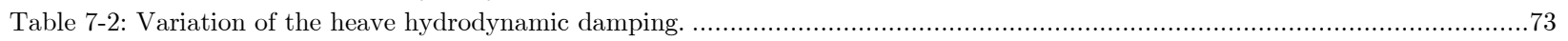

Table 8-1: Optimal Floating Breakwater lay-out and performance for several wave periods................................................82 


\section{USED SYMBOLS}

\section{Roman}

\begin{tabular}{|c|c|c|c|c|}
\hline$a_{n n}$ & : & Hydrodynamic added mass in direction (nn) & $\left(\mathrm{kN} \cdot \mathrm{s}^{2} / \mathrm{m}\right)$ & $(\mathrm{t})$ \\
\hline$a_{44}$ & : & Hydrodynamic added moment of inertia for roll & $\left(\mathrm{kN} \cdot \mathrm{m} . \mathrm{s}^{2}\right)$ & $\left(\mathrm{t} . \mathrm{m}^{2}\right)$ \\
\hline$b_{n n}$ & $:$ & Hydrodynamic damping direction (nn) & $(\mathrm{kNs} / \mathrm{m})$ & $(\mathrm{t} / \mathrm{s})$ \\
\hline$b_{44}$ & : & Hydrodynamic damping for roll & (kN.m.s) & $\left(\mathrm{t} . \mathrm{m}^{2} / \mathrm{s}\right)$ \\
\hline$c_{n n}$ & : & Hydrodynamic spring stiffness in direction $(\mathrm{nn})$ & $(\mathrm{kN} / \mathrm{m})$ & $\left(\mathrm{t} / \mathrm{s}^{2}\right)$ \\
\hline$d_{m}$ & : & Damping of the mooring system & $(\mathrm{kNs} / \mathrm{m})$ & $(t / s)$ \\
\hline$g$ & : & Gravitational acceleration & & $\left(\mathrm{m} / \mathrm{s}^{2}\right)$ \\
\hline$k$ & : & Wave number & & $(\mathrm{rad} / \mathrm{m})$ \\
\hline$k_{m}$ & : & Spring stiffness of the mooring system & $(\mathrm{kN} / \mathrm{m})$ & $\left(\mathrm{t} / \mathrm{s}^{2}\right)$ \\
\hline$m$ & : & Structural mass of the floating breakwater & $\left(\mathrm{kN} . \mathrm{s}^{2} / \mathrm{m}\right)$ & $(\mathrm{t})$ \\
\hline$y_{b}$ & : & Horizontal lever arm relative to the rotation point & & $(\mathrm{m})$ \\
\hline$y$ & : & Displacement in the $y$-direction & & $(\mathrm{m})$ \\
\hline$\dot{y}$ & : & Velocity in the $y$-direction & & $(\mathrm{m} / \mathrm{s})$ \\
\hline$\ddot{y}$ & : & Acceleration in the $y$-direction & & $\left(\mathrm{m} / \mathrm{s}^{2}\right)$ \\
\hline$z_{b}$ & : & Vertical lever arm relative to the rotation point & & $(\mathrm{m})$ \\
\hline$z$ & : & Displacement in the z-direction & & $(\mathrm{m})$ \\
\hline$\dot{z}$ & : & Velocity in the z-direction & & $(\mathrm{m} / \mathrm{s})$ \\
\hline$\ddot{z}$ & : & Acceleration in the z-direction & & $\left(\mathrm{m} / \mathrm{s}^{2}\right)$ \\
\hline$A_{s}$ & : & Submerged area of the floating breakwater section in the xy-plane & & $\left(\mathrm{m}^{2}\right)$ \\
\hline$A_{w}$ & : & Submerged volume of the floating breakwater section. & & $\left(\mathrm{m}^{3}\right)$ \\
\hline$C_{3}$ & : & Vertical wave pressure distribution coefficient & & $(-)$ \\
\hline$F_{v}$ & : & Resultant of the vertical dynamic wave force & & $(\mathrm{kN})$ \\
\hline$F_{h}$ & : & Resultant of the horizontal dynamic wave force & & $(\mathrm{kN})$ \\
\hline$H$ & : & Wave height & & $(\mathrm{m})$ \\
\hline$I_{x x}$ & : & Mass moment of inertia about $\mathrm{G}$ & & $\left(\mathrm{m}^{4}\right)$ \\
\hline$K$ & : & Moment around $\mathrm{O}$. & & $(\mathrm{kNm})$ \\
\hline$L_{w}$ & : & Length of the floating breakwater element & & $(\mathrm{m})$ \\
\hline$O$ & : & Rotation origin on the waterline & & $(-)$ \\
\hline$G$ & : & Centre of gravity of the floating breakwater section & & $(-)$ \\
\hline$\overline{G M}$ & : & Transverse metacentric height & & $(\mathrm{m})$ \\
\hline$\overline{B M}$ & : & Distance between centre of buoyancy and metacentre & & $(\mathrm{m})$ \\
\hline$\overline{B G}$ & : & Distance between the centres of gravity and buoyancy & & $(\mathrm{m})$ \\
\hline$\overline{O G}$ & : & Distance of centre of gravity below the waterline & & $(\mathrm{m})$ \\
\hline$T$ & : & Wave period & & (s) \\
\hline$T_{1}$ & : & Draft of the floating section of the breakwater. & & $(\mathrm{m})$ \\
\hline$T_{2}$ & : & Draft of the screen underneath the floating section of the breakwater & & $(\mathrm{m})$ \\
\hline$T_{s}$ & : & Draft of the representative vessel & & $(\mathrm{m})$ \\
\hline$Y_{w}$ & : & Width of the floating section of the floating breakwater & & $(\mathrm{m})$ \\
\hline
\end{tabular}




\section{Greek}

$\begin{array}{ll}\nabla & : \\ \delta \pi & :\end{array}$

$\varepsilon_{n \zeta} \quad:$

$\mathcal{E}_{\text {tot } ; \max }:$

$\phi \quad$ :

$\dot{\phi} \quad$ :

$\ddot{\phi} \quad$ :

$\zeta_{a} \quad:$

$\zeta_{T} \quad:$

$\zeta_{w n}^{*} \quad:$

$\dot{\zeta}_{w n}^{*} \quad:$

$\ddot{\zeta}_{w n}^{*} \quad:$

$\omega_{n}:$

$\lambda \quad:$

$\rho \quad:$
Volume of water displacement of the body in an equilibrium state

The additional phase shift to determine the maximum total transmitted wave amplitude

Motion-related phase angle

Phase shift where the maximum amplitude of the transmitted wave occurs.

Rotation in the $\phi$-direction

Velocity in the $\phi$-direction

Acceleration in the $\phi$-direction

Incoming wave amplitude at the sea side of the floating breakwater

Transmitted wave amplitude to the harbour side of the floating breakwater.

Reduced wave elevation in the n-direction.

Reduced velocity of the water particles in the n-direction.

Reduced acceleration of the water particles in the n-direction.

Directional natural frequency

Wave length

Water density $\left(\mathrm{m}^{3}\right)$

(rad)

(rad)

$(\mathrm{rad})$

$(\mathrm{rad})$

$(\mathrm{rad} / \mathrm{s})$

$\left(\mathrm{rad} / \mathrm{s}^{2}\right)$

(m)

(m)

(m)

$(\mathrm{m} / \mathrm{s})$

$\left(\mathrm{m} / \mathrm{s}^{2}\right)$

$(\mathrm{rad} / \mathrm{s})$

(m)

$\left(\mathrm{kN} / \mathrm{m}^{3}\right)$ 


\section{Chapter 1}

\section{Introduction}

Developing large floating structures for all kind of purposes has become more interesting in the past decade since the demand fur such structures increased significantly. The focus of this report will be on the container shipping business. A Floating Transhipment Container Terminal (FTCT) is an interesting structure in this business from a practical as well as an economical point of view.

- $\quad$ Due to the size of the modern container vessels it is important to create harbours deep and wide enough able to serve these kinds of vessels.

- $\quad$ The number of harbour calls can be reduced when container terminals can be placed at strategic locations.

From previous studies it appeared that the efficiency of floating harbours is affected by wave attack. A floating breakwater is necessary to increase the efficiency rate and to create a safe haven for vessels when the weather conditions become bad.

Although many studies were performed to determine the performance of floating breakwaters with various designs, no floating breakwater has been developed yet that meets the necessary requirements. The breakwaters that have been applied in real time situations were all designed to attenuate waves at a specific location with moderate wave conditions. For the purpose of the FTCT, a breakwater is necessary which is able to attenuate waves of a wide wave spectrum at open ocean areas.

A floating harbour has a complex dynamic behaviour. In contrast to a normal harbour, where only ship motions occur, a floating harbour will be completely influenced by the wave conditions. Lots of structural and hydraulic factors influence the hydrodynamic behaviour of the different elements in a floating harbour. Determining the relations and the influence of these factors on the wave attenuating capacity of the floating breakwater is the main objective of this study.

With this knowledge a model can be developed that can be used as a design tool to determine the magnitude of the floating breakwater elements in an early stage of the design process. The model can answer the question whether or not floating breakwaters are suitable for a wide application. So, apart from the engineering problem to assess the importance of the structural variables, it has to be determined whether the floating breakwater can be a feasible solution from a theoretical point of view.

It is therefore important to structuralize the problem to be a base for later, more detailed assessment and model testing. 
The structure of the report is as follows:

Chapter 2: A short overview of the most important breakwater types and their differences with a floating breakwater is discussed in chapter 2 .

Chapter 3: $\quad$ Chapter 3 will give an introduction on the past performance of floating breakwaters and possible applications of these structures in the near future. Furthermore, the main conclusions of previous studies on this topic are summarized in this section.

Chapter 4: The problem definition and the objectives of this thesis are presented in chapter 4 .

Chapter 5: $\quad$ The boundary conditions and requirements will be discussed in chapter 5 .

Chapter 6: $\quad$ In chapter 6 the Rectangular Floating Breakwater Model (ReFBreak) will be presented. Assumptions, calculation parameters and the structure of the model will be presented in this chapter.

Chapter 7: $\quad$ Evaluation of the calculation results and a parameter analysis will be discussed in chapter 7.

Chapter 8: A case study is presented in chapter 8 in order to create an insight in how the ReFBreak-model should be used in real design applications.

Chapter 9: Finally, in chapter 9, conclusions will be drawn and recommendations on further research will be given. 


\section{Chapter 2}

\section{Breakwaters in a nutshell}

Since time immemorial, harbours played a deciding role in the extent of prosperity for entire populations. In the early history, naturally sheltered locations (like bays and estuaries) were used as a haven for ships. Soon these sheltered locations, where little wave attack was encountered, became the centres of trade. When the economical importance of harbours increased further more, these harbours became the centres of society as well. Nowadays, space has become very scarce in coastal zones and around harbour areas in particular. However, technological developments made it possible to extend the harbours into the ocean. Often, artificial breakwaters are used to create the sheltered area where harbour activities take place.

The primary function of a breakwater is to attenuate waves to an acceptable level or eliminate their effects altogether. It creates a sheltered region in order to prevent damage to shorelines, harbours, and other natural or man-made structures. Although there are several types of breakwater structures, one can roughly distinguish three main types of breakwaters, which are:

\section{Conventional (mound) type of breakwaters}

Mound types of breakwaters are actually no more than large heaps of loose elements, such as gravel and quarry stone or concrete blocks.

\section{Monolithic type of breakwaters}

Monolithic types of breakwaters have a cross section designed in such a way that the structure acts as one solid block. In practice, one may think of a caisson, a block wall, or a masonry structure. Generally this kind of structure is used when space is scarce and local water depths are relatively large.

\section{Composite type of breakwaters}

A composite type of breakwater is a combination of the conventional and monolithic type of breakwater. When water depths get larger, this kind of structures is often preferred from an economical point of view.

Although the designs of the breakwaters (fig.2-1) differ form one another, a lot of similarities can be distinguished. They are all built to block the incoming waves and to dissipate or reflect the wave energy. They are all fixed structures, designed for a specific location. Bottom-founded structures are limited to a certain maximum water depth since these structures are impossible in deep water environments from a technical as well as an economical point of view.

From a military, a humanitarian, a technical and an economical point of view, a new type of breakwater is needed to overcome the restrictions that are associated with fixed breakwaters. This new type of breakwater has to be rapidly installed, transportable, (re-)usable at several locations with different wave conditions and applicable in deep water areas. Several types of unconventional breakwaters have been developed in the past in order to meet these demands, including the floating breakwater. 
Although a lot of (theoretical and practical) research has been done on a wide variety of floating breakwater concepts, the appliance of floating breakwaters in real situations is very limited. The complex contribution of the dynamic response to the total wave transmission is the main reason for this. This dynamic response makes a floating breakwater only suitable for a small frequency range. Figure 2-1 shows the phenomena that contribute to the two-dimensional wave transmission for several types of breakwaters.

A floating breakwater that performs well in a wide frequency range has not been developed yet. This report will concentrate on several aspects in order to design a floating breakwater that is capable of attenuating waves in a frequency range, wide enough to satisfy the harbour demands. A numerical model will be developed to prove the contribution of the most basic design elements such as draft, width, weight and mooring line stiffness to the performance of the floating breakwater. This numerical model will be used as a design tool to develop a solution that will meet the requirements.
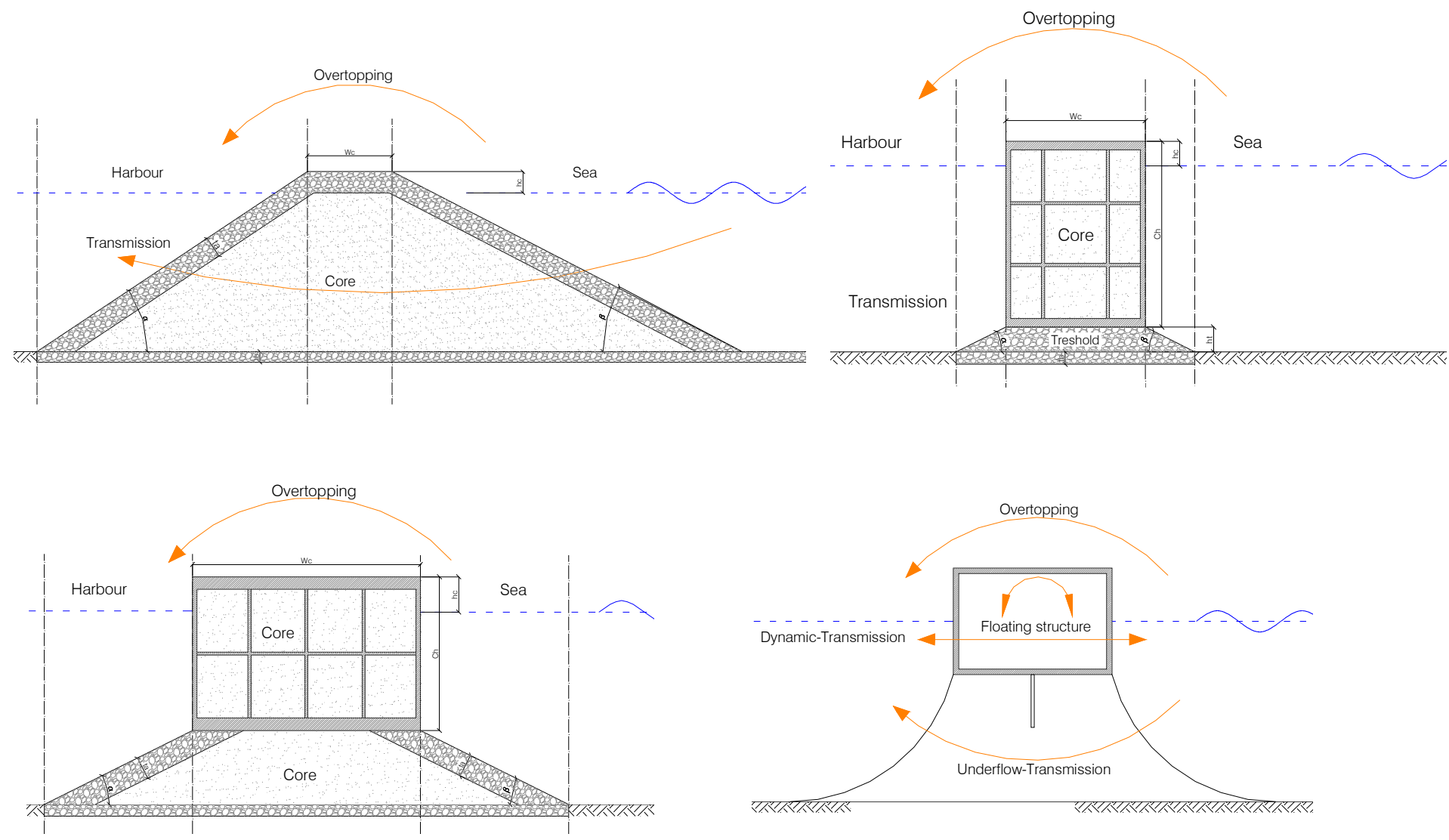

Figure 2-1: Several breakwater structures.

From left to right, top to bottom: Rubble mound breakwater, Caisson breakwater, Composite breakwater, Floating breakwater. 


\section{Chapter 3}

\section{Past performance and research}

Ever since progressive engineers came up with the idea of creating floating structures into the sea, around 100 years ago, many studies and model tests were performed to develop a floating breakwater. Although the first engineers used the trial-and-error approach to test their creations (Mr.Thuillard-Froideville in 1884 to protect the harbour of Le Havre), research on this topic professionalized soon after the Second World War.

A lot of designs were laboratory tested and checked with numerical calculations. In this section, a short review will be given of the floating breakwaters that have been built and the possibilities for future floating breakwaters. Past model testing will be discussed in this section in order to describe the problems that have been encountered in the past.

\subsection{Why floating breakwaters?}

The main reasons to apply unconventional types of breakwaters, and floating breakwaters in particular, are the technical and economic restrictions related to the monolithic and conventional types of breakwaters. Besides these restrictions, there are other arguments that are encouraging the development of floating breakwaters, as illustrated in figure $3-1$.

Although the advantages of a floating breakwater seem to be rather obvious, a couple of disadvantages can be distinguished as well. Performance, costs and human confidence in a floating breakwater are the primary counter arguments.

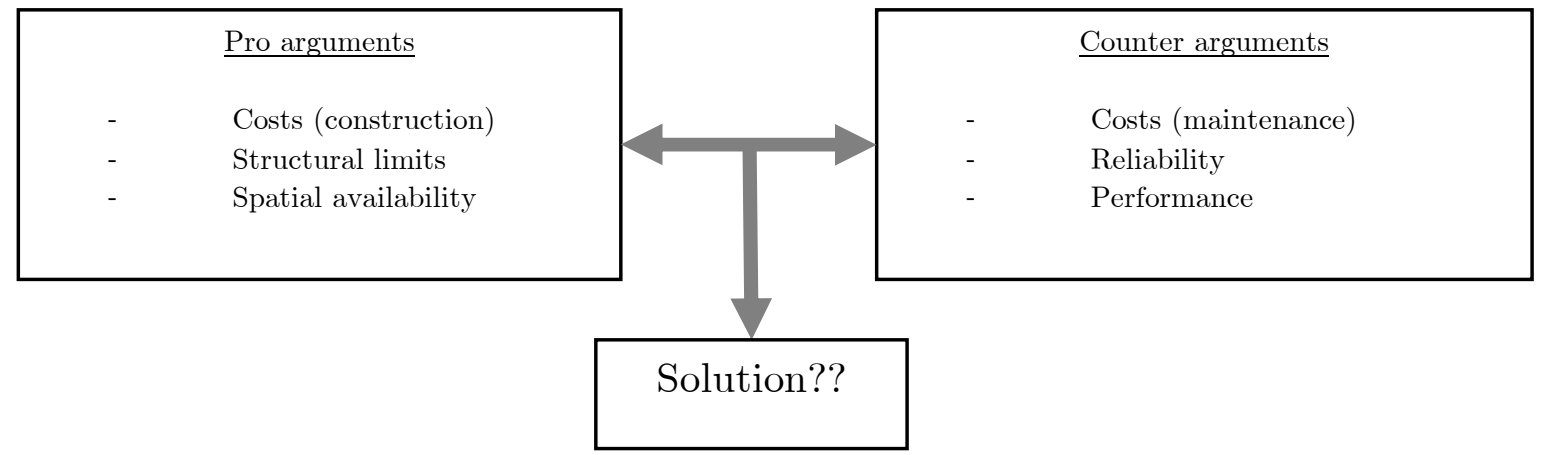

Figure 3-1: The pros and cons on developing a floating breakwater. 


\subsubsection{Economic arguments}

The advantages and disadvantages of the use of floating breakwaters have a common origin: economics. While maintenance ${ }^{1}$ costs are mentioned in figure 3-1 as a disadvantage of a floating breakwater, the independence of realisation cost related to the water depth is a big advantage in favour of the floating breakwater.

To illustrate this assumption, a construction cost calculation was made to determine the optimal breakwater construction for several water depths. This primary study only involves the construction costs.

The construction costs of the conventional, the caisson and the composite breakwater are based on the studies done by $[\text { Schepers }]^{2}$ and [Lenting $]^{3}$.

Simplified cross sections are used to find a first approximation of the optimal structure for several water depths. The simplified cross sections that were used in this cost analysis were shown in figure 2-1.

Figure 3-2 shows the relation between construction costs and water depth. In previous reports $\left[\mathrm{d}^{\prime} \text { Angremond }\right]^{4}$ it was already stated that the conventional breakwater, from an economic point of view, will only be preferable until a water depth of around $8 \mathrm{~m}$. In depths ranging from $8 \mathrm{~m}$ to $20 \mathrm{~m}$, a caisson breakwater will be the best solution. And after that, up to a depth of $30 \mathrm{~m}$, the composite type of breakwater is preferable. Figure 3-2 shows almost the same results. At this stage, the cost of the floating breakwater is unknown. Since the construction costs of a floating breakwater will hardly increase with increasing depths, the line in the figure will be an almost horizontal line.

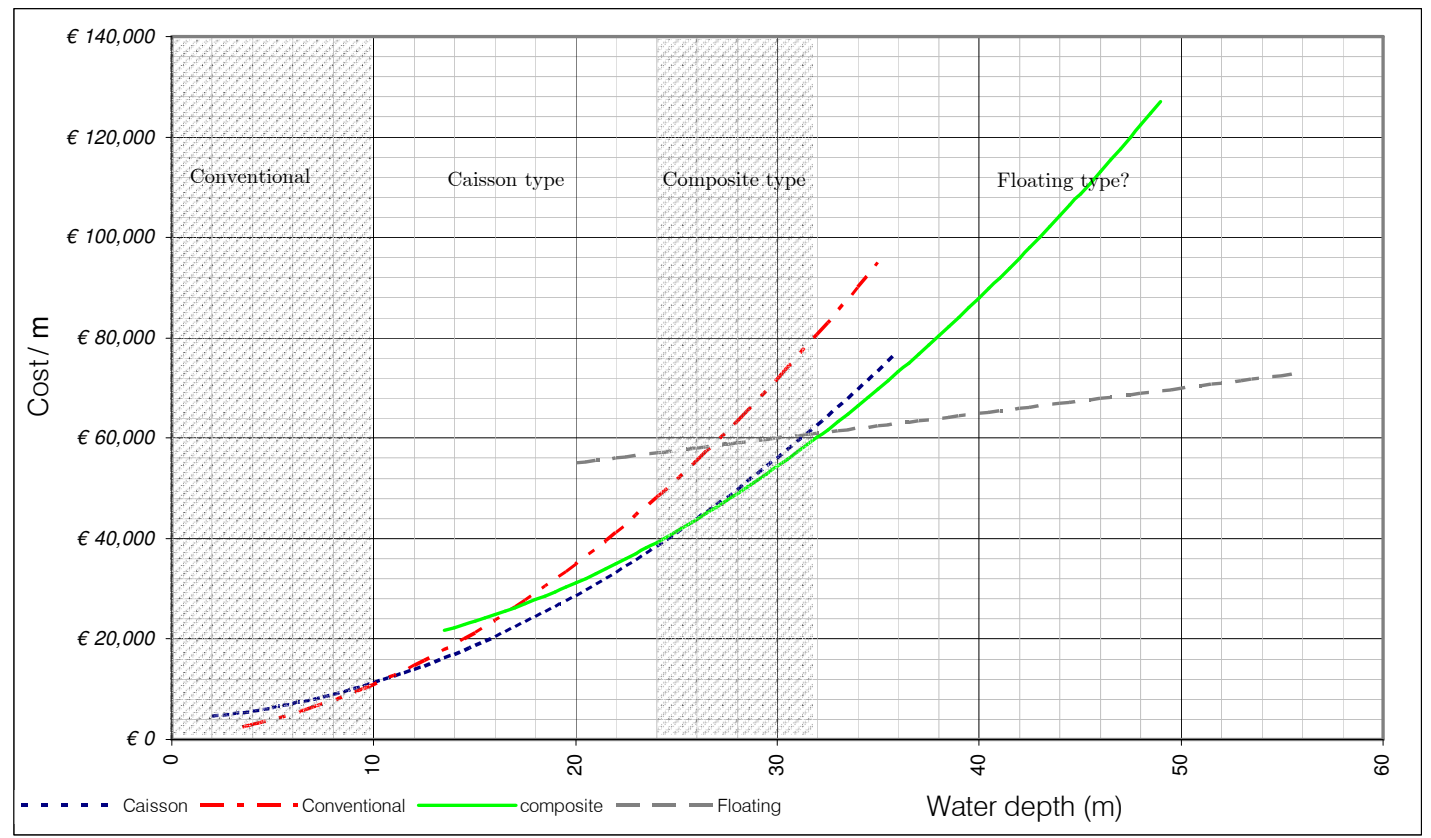

Figure 3-2: Comparison of construction cost /m1 depending on the water depth.

For real situations, specific site conditions may alter the results drastically. Construction costs depend on the rate of downtime due to wave climate and tidal height conditions. Construction costs are largely depending on the available weather window determined by wave and tidal conditions.. Moreover, the feasibility of a caisson solution depends largely on the stability of the foundation and, in particular, the sensitivity of the subsoil to liquefaction.

${ }^{1}$ Tsinker, G. (1995). 'Marine Structures Engineering: Specialized Applications', Chapman and Hall, New York.

${ }^{2}$ Schepers, M.R. (1998). Msc thesis 'Kosten beschouwing van conventionele golfbrekers met betonelementen', Delft University of Technology.

${ }^{3}$ Lenting, F.V. (2003). Msc thesis 'Een alternatief voor een verticale wand golfbreker', Delft University of Technology.

${ }^{4}$ d'Angremond, K. and Tutuarima, W.H. (1998). Coastal Engineering 1998, Volume 2, Conference proceedings. 


\subsubsection{Technical arguments}

\section{Construction}

Costs are not the only reason why conventional breakwaters are not preferable when water gets too deep. Local soil conditions and structural stability do also influence the limits of design. A huge structure will result in tremendous pressures on the subsoil as well as stability problems when the slopes become too steep. A floating breakwater can be more feasible in poor soil conditions than a heavy fixed breakwater since the subsoil pressure is virtually non-existent, however, the floating breakwater does have to be anchored to the sea bottom.

Floating breakwaters can be easily moved and rearranged due to their transportability, reusability and flexibility in design. Due to this quality, a floating harbour can be adapted and rearranged easily when needed.

\section{Performance}

Floating breakwaters can effectively attenuate wave heights of a limited frequency range. They are less effective for relative longer waves than fixed structures are. The structure will show a simultaneous movement with the water surface at a certain frequency range. Of course this depends on a range of factors, like the weight of the structure and the mooring line stiffness, etc.

Floating breakwaters have minimal interference with water circulation, sediment transport and fish migration.

Floating breakwaters can be easily moved and rearranged due to their transportability, reusability and flexibility in design.

The way a floating breakwater fails has to be taken into consideration. A damaged, bottom-founded structure will show a certain 'failure warning' and will not suddenly be destroyed. Floating breakwaters however are susceptible to a sudden structural failure during catastrophic storms ${ }^{5}$.

A floating breakwater can become a threat for the harbour facilities if the mooring fails.

${ }^{5}$ Tsinker, G. (1995). 'Marine Structures Engineering: Specialized Applications', Chapman and Hall, New York. 


\subsection{Past Performance}

The development of floating, transportable breakwaters got a real boost when the necessity arose to land men and materials during the Normandy invasion of Wold War II. Two types of breakwaters were used for that purpose. The first types were concrete barges, transported from Great Britain. These barges were positioned just off-shore and were sunken down in order to create a bottom-founded breakwater. The second types of breakwaters were floating structures with a cruciform cross section. These 'Bombardon' floating breakwaters were steel structures arranged in lines along the Normandy coast.

The 'Bombardon' floating breakwaters served their purpose during the invasion but failed after 9 days during a storm which created stresses eight times higher than what they were designed for.

After this experience, the faith in the reliability of the floating breakwater was gone for many years. In the 1950's, the US navy saw the potential of these structures to protect small craft and marine structures against open-ocean waves. A manageable, transportable, reusable floating breakwater was investigated that would provide a sheltered environment during several military or humanitarian operations. Serious development of this type of floating breakwater lasted until the 1980's, when several rapidly installed floating breakwaters (RIBS) were tested at full scale. These breakwaters, developed for military purposes were designed to attenuate wave heights in a certain part of the wave spectrum to an acceptable level.

Besides the military-orientated floating breakwaters, some commercial breakwaters have been developed as well. Small-structured floating breakwaters, designed to protect small scale marinas against short crested waves, are already in wide use. These kinds of structures are used all around the world in relatively moderate wave conditions. Although these structures are quite successful, the appliance of large-scale floating breakwaters is not yet that common.

One of the few interesting examples, designed to defend a large harbour, is the pier extension of Port Hercule in Monaco. In 2002 'La digue semi-flottante' was installed as a pier extension in Monaco, in approximately $55 \mathrm{~m}$ of deep water. An enormous caisson, 352 meters long, with a main body $28 \mathrm{~m}$ wide, a total depth of 19 $\mathrm{m}$ and a draft of $16 \mathrm{~m}$ was installed. It is multifunctional and, as a permanent structure, it has to withstand design storm conditions during its expected lifetime of 100 years.

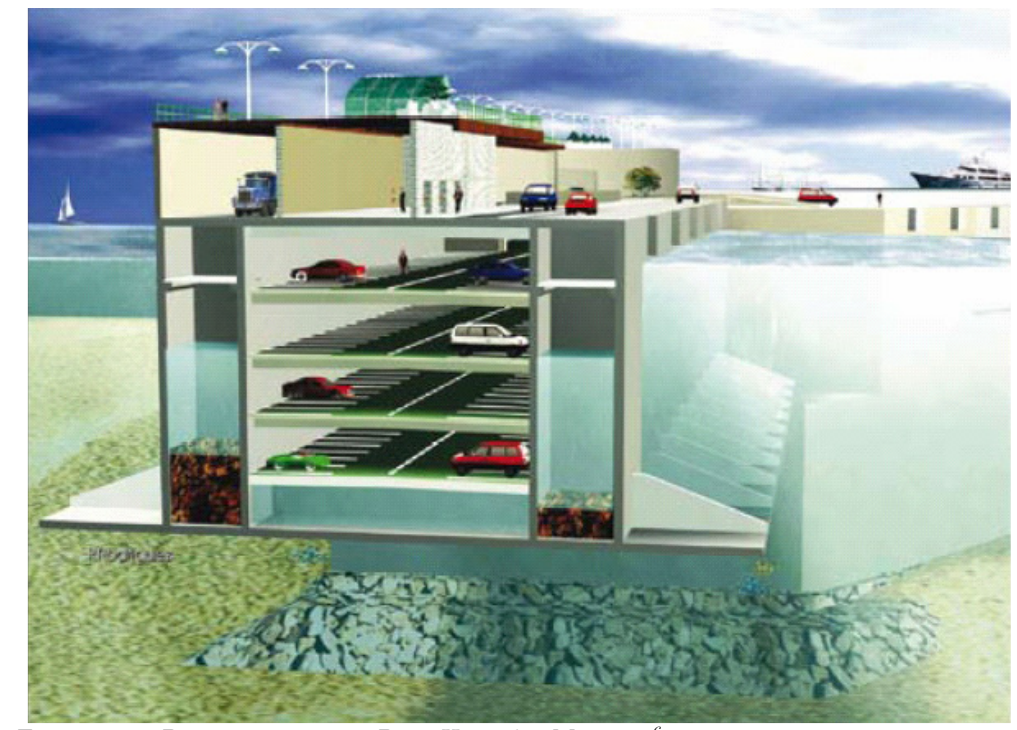

Figure 3-3: Pier extension at Port Hercule, Monaco. $^{6}$

\footnotetext{
${ }^{6}$ Picture from BBR technologies, 'BBR News special edition 2006'.
} 
Floating breakwaters, as they have been applied in real situations, can be split into three categories:

- $\quad$ Light-weight floating breakwaters which are easy to reuse and to transport. Service times vary from several hours to a couple of days. Example: Rapidly Installed Breakwater System (RIBS) as it is used by the US Navy to load and unload troops into small landing vessels.

- $\quad$ Light-weight floating breakwaters with a semi-permanent character. Service time can take up to 30 years. This type of floating breakwater is very common in small-scale marinas. Example: U-block, which is used to defend several marinas in Greece. The U-block floating breakwater consists of concrete caissons, filled with polystyrene, that are connected to one another by cables. This type of floating breakwater can be transported very easily if wave conditions exceed the design conditions or when it has to be reused at another location.

- $\quad$ Heavy-weight floating breakwaters with a permanent character. Service time can take up to 100 years. This type of floating breakwater is only applied if water depth or soil conditions do not allow a fixed breakwater and the wave conditions are moderate. Example: Monaco semi-floating breakwater. The structural dimensions of this pier extension are of such a level that the structure can cope with the Mediterranean wave spectrum very easily. However, the structure becomes less transportable and reusable due to these structural dimensions.

An overview of the main characteristics of the structures, as they were mentioned above, is given in table 3-1.

\begin{tabular}{|c|c|c|c|}
\hline & \multicolumn{3}{|c|}{ Concept } \\
\hline & Monaco Breakwater & U-block & $R I B S$ \\
\hline Costs & -- & + & ++ \\
\hline Flexibility & -- & + & - \\
\hline Construction time & - & + & ++ \\
\hline Spectrum width & ++ & - & -- \\
\hline Durability & + & + & - \\
\hline Transportability & $+/-$ & $+/-$ & ++ \\
\hline Reusability & - & + & ++ \\
\hline Designer & Doris engineering, Paris & FDN Civil engineering, TU Delft & $\begin{array}{l}\text { U.S. Army Engineer Research } \\
\text { and Development Center }\end{array}$ \\
\hline Materials & Reinforced concrete & $\begin{array}{l}\text { Reinforced concrete with a core of } \\
\text { polystyrene }\end{array}$ & $\begin{array}{l}\text { Steel frames with flexible } \\
\text { membranes }\end{array}$ \\
\hline Design life & 100 years & 30 years & Temporary \\
\hline Length & $352 \mathrm{~m}$ & $25 \mathrm{~m}$ (each element) & $77 \mathrm{~m}$ (each leg) \\
\hline Width & $28 \mathrm{~m}$ & $4.50 \mathrm{~m}$ & $2.40 \mathrm{~m}$ \\
\hline Draft & $16 \mathrm{~m}$ & $7.30 \mathrm{~m}$ & $4 \mathrm{~m}$ \\
\hline Weight & 454 tons $/ \mathrm{m}$ & 5.40 tons $/ \mathrm{m}$ & \\
\hline Station keeping & Ball-and-socket joint \& Cables & Chain / cable & 3-points, chain \\
\hline Design wave height & & $2 \mathrm{~m}$ & 0.50 to $1.50 \mathrm{~m}$ \\
\hline Application areas & Cruise ship terminal & Small-scale marinas & Military landing operations \\
\hline
\end{tabular}

Table 3-1: Comparison of floating breakwater designs.

None of the floating breakwater systems, as they have been applied in the past have the qualities of both being applicable in a wide wave spectrum and to be reusable at another location than they were originally designed for. 


\subsection{Possible applications in the near future}

In the previous section it became clear that there is a wide range of sources with a maritime origin that emphasize the need to develop a floating breakwater. During this research, the focus will be on the defence of the so-called Floating Transhipment Container Terminals (FTCT) against wave attack.

International container shipping is one of the most dynamic economic sectors of the past few years. Between 1990 and 2005 the container trade at the world's ports expanded by less than $10 \%$ p.a. on average ${ }^{7}$. The expected annual growth of the international container shipping will be around $9 \%$ up to 2015 .

The reason for this growth is twofold. On the demand side, the increasing division of welfare in the world gives a rise in importance of goods, eminently suited to transport by container. Higher efficiency of the loading and unloading processes and the increase in size of container vessels contributed to the growth at the supply side.

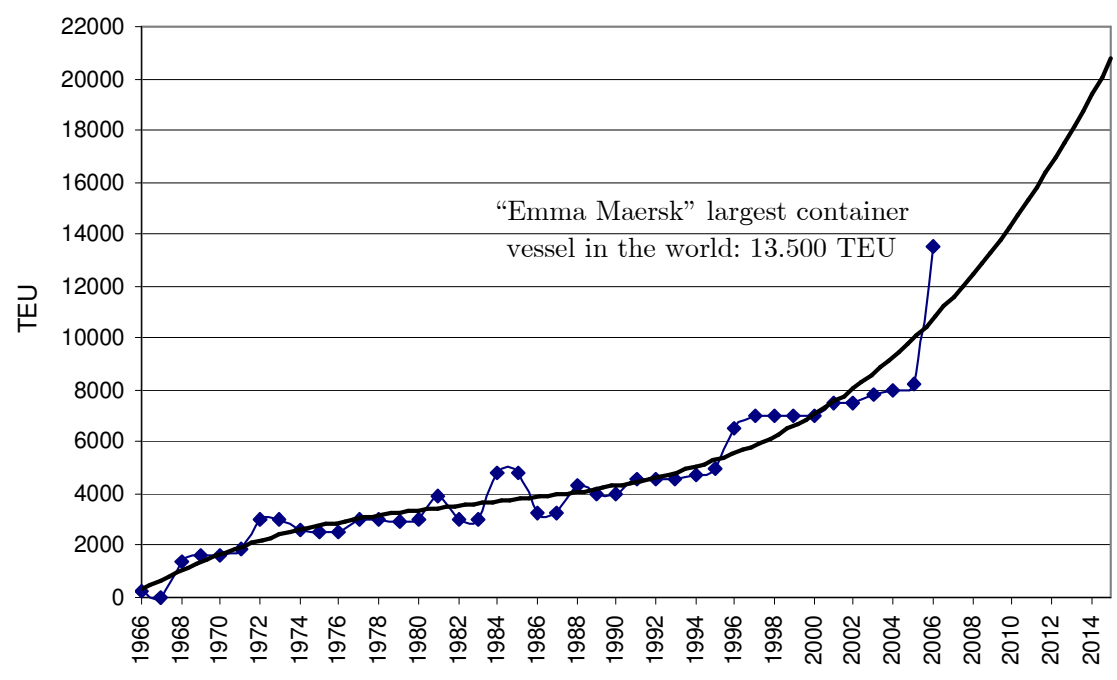

Figure 3-4: Maximum ship size by year of construction (until October 2006).

The increase of container handling in the world makes the development of container terminals necessary. Existing harbours already increased their container handling capacity or transferred their general cargo terminals into container terminals.

The introduction of the mega vessel as well as the increasing efficiency of shipping lines demands for deep water container terminals. An increase of efficiency is gained when container vessels are able to reduce their number of port calls. This is achieved with the 'terminal-feeder' system. Mega (deep-sea) container vessels travel between the deep water container terminals where they are offloaded and loaded. Smaller feeder vessels transport the containers to their final destinations.

Feeder vessels are container ships with an average capacity of carrying 300-500 $\mathrm{TEU}^{8}$. Feeders collect containers from different ports and transport them to central (deep-water) container terminals where they are loaded to bigger vessels. On the way back, the feeders are loaded with other containers that have to be transported to a certain port. Throughout the years so-called feeder lines were created on which ship-owners are transporting containers over a predefined route on a regular basis.

\footnotetext{
${ }^{7}$ Heymann, E. (2006). 'Container shipping. Overcapacity inevitable despite increasing demand', Deutsche Bank Research

${ }^{8}$ The abbreviation TEU stands for Twenty feet Equivalent Unit. 1 TEU is a $20 \mathrm{ft}$ container.
} 
An example of such a terminal-feeder system, and the feeder routes involved with it, is shown in figure 3-5. In this case, the terminal is the port of Rotterdam. From Rotterdam, feeder vessels transport the containers to ports all over Europe.

The necessity of deep water container terminals, combined with the technical and economical limitations as they were mentioned in the previous section, gave rise to the development of the Floating Transshipment Container Terminal (FTCT). A FTCT is a floating structure where container vessels can load and un-load their containers. Besides this transfer function, the FTCT must be able to store large numbers of containers, waiting for their transshipment.

Handling containers in a harbour is not possible when ship motions get too large. In a floating harbour, motions will cause even more problems, since both ship and floating harbour will react on the waves. To guarantee optimal harbour efficiency, the mutual motions of vessel and harbour may not exceed a certain maximum.

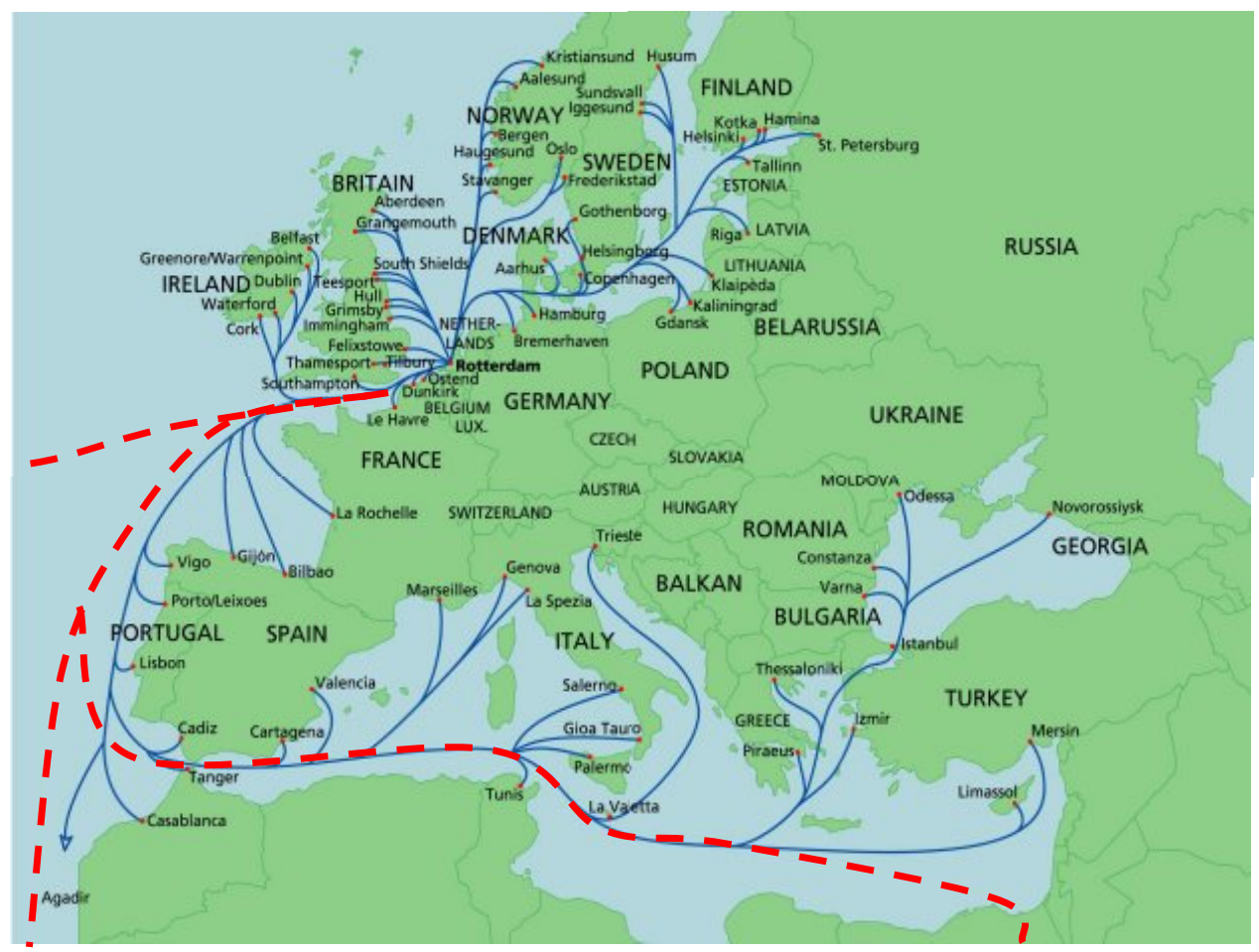

Figure 3-5: Container feeder transport system for the port of Rotterdam ${ }^{9}$

\footnotetext{
${ }^{9}$ European Container Terminals ECT, www.ect.nl.
} 


\subsection{Performed research on dynamic behaviour}

A lot of research has been done on the hydrodynamic behaviour of floating breakwaters. The main focus of all these studies has always been to obtain transmission coefficients that are as small as possible. The transmission coefficient is the ratio between the wave height at the leeward (harbour) side of the floating breakwater relative to the wave height of the incident wave.

In order to obtain satisfactory results, many designs were model-tested.

Although the tested models do vary in design, the common research topics can be split into the influence of the structural design and the structural dynamics on the wave attenuating capacity of the structure. The major aspects of the structural design that have been tested:

$\begin{array}{ll}\text { - } & \text { Shape } \\ \text { - } & \text { Width of the floating section of the structure } \\ \text { - } & \text { Draft of the structure } \\ \text { - } & \text { Permeability of the structure } \\ \text { - } & \text { Station keeping }\end{array}$

All these structural factors influence the (hydro)dynamic behaviour of the floating breakwater. In some of the model tests, the vertical, horizontal and rotational oscillations were treated separately in order to understand the individual influence of the design parameters on the different motions.

Interesting results of the studies that were performed are given in appendix A.

In their reports, Tolba ${ }^{10}$ and Silander ${ }^{11}$ proved that a heave motion floating breakwater will perform better when it is not affected in its vertical degree of freedom. Sway motion on the other hand has a negative contribution on the attenuating capacity of the floating breakwater.

Silander proved that a free-motion floating breakwater performs better at a certain frequency range when the structural width is optimal (fig.3-6). In this figure, optimal floating breakwater performance (cross-hatched area) is gained when a free motion floating breakwater is considered with a certain width $\left(\mathrm{K}_{\mathrm{t}}=\right.$ wave transmission coefficient). This conclusion results in the idea that the influence of the mooring system must be kept as small as possible.

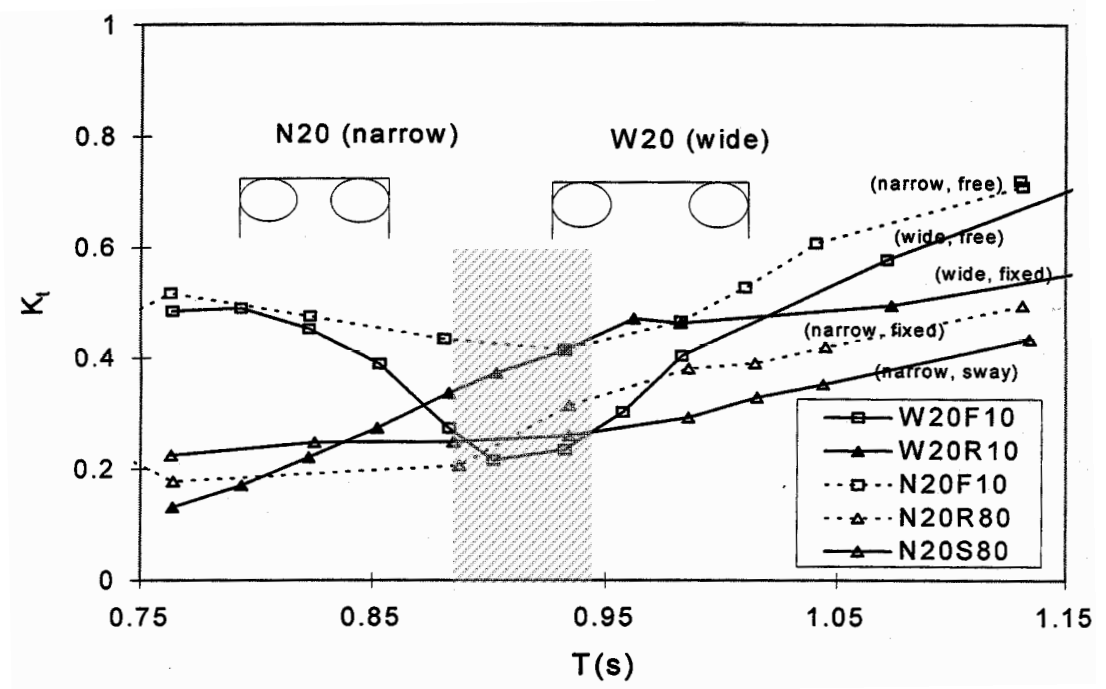

Figure 3-6: Performance of several types of structures.

\footnotetext{
${ }^{10}$ Tolba, E.R.A.S. (1998). 'Behaviour of floating breakwaters under wave action', PhD thesis, Suez Canal University of Egypt

${ }^{11}$ Silander, J., (1999), 'Floating breakwater and environment', Helsinki University of Technology.
} 
The draft and the mass of the structure are related to one another when the structural width is kept constant. Increasing the draft with a screen has a positive effect on the wave transmission as Tolba concludes in his report.

In all studies that were performed in the past, the same conclusions are conducted on the behaviour and the performance of the floating breakwater.

Williams and McDougal ${ }^{12}$ conducted a two-dimensional analysis of a floating breakwater with a rectangular cross-section. Since they assumed beam waves and an infinitive structural length, the motions of the floating breakwater were described to be two-dimensional in the xz-plane. The mooring lines were schematized as linear springs. Besides the two-dimensional representation of the model, Williams and McDougal assumed regular, small amplitude waves with constant periods. When considering the irregular oblique nature of ocean waves, the assumptions done in this study can be invalid. However, the results give a good representation on the effectiveness of a floating breakwater in different wave conditions.

The most important conclusions of this study are:

- $\quad$ The breakwater is most effective near the surge natural frequency

- The performance of the structure is optimal when the diffracted and radiated waves are of the same magnitude, but with a phase difference of .

Similar results were obtained by Yamamoto and Yoshida ${ }^{13}$ as they conducted experimental tests for two kinds of floating breakwater types in order to validate theory-based calculations. The first type was a three-circle cylinder with a cross-section of three adjacent circles. The second type had a rectangular cross-section. As was the case in the study performed by Williams and McDougal, Yamamoto and Yoshida assumed linear spring stiffness for the mooring lines and beam incident waves.

The test results yielded a good agreement with the theoretical models. Yamamoto and Yoshida concluded that even zero transmission coefficients could be obtained at either very low or high frequencies. The best performance was gained for waves with frequencies near the natural frequency of the structure.

Evans and Linton ${ }^{14}$ performed a study on another kind of floating breakwater: the submerged floating breakwater. A theoretical model, based on the linear wave theory was used to determine the behaviour of a long, fully submerged cylinder in regular beam waves. Evans and Linton concluded that a zero transmission coefficient can be obtained at what they called the 'tuning-frequency' of the floating breakwater. With the tuning frequency they mean that the natural frequency of the floating breakwater can be tuned in such a way that the performance is optimal.

Besides floating breakwaters with cylindrical cross-sections, model tests and calculations were performed for so-called vertical wall breakwaters or wave screens. Vertical wall breakwaters reduce the wave energy by creating vortices due to their permeability. Screen draft and permeability are important factors for these kinds of structures.

Isaacson ${ }^{15}$ and Gardner ${ }^{16}$ performed model tests to determine the influence of the screen draft and the permeability on the total wave transmission. The experiments proved that screens with a porosity of 10 and

${ }^{12}$ Williams, A.N. and McDougal, W.G. (1996). 'A dynamic submerged Breakwater'. Journal of Waterway, Port, Coastal and Ocean Engineering. ASCE

${ }^{13}$ Yamamoto, T. and Yoshida, A. (1979). 'Large wave tank tests on taut-moored breakwaters'. Coastal structures '79, Vol.II, ASCE.

${ }^{14}$ Evans, D.V. and Linton, C.M. (1991). 'Submerged floating breakwaters'. Journal of Offshore Mechanics and Arctic Engineering.

${ }^{15}$ Isaacson, M., Premasiri, S. and Yang, G. (1998). 'Wave Interactions with Vertical Slotted Barrier'. ASCE Journal of Waterway, Port, Coastal and Ocean Engineering. 
$20 \%$ resulted in transmission coefficients of 0.60 and 0.65 respectively. Furthermore, they proved that increasing the screen draft results in less wave transmission.

Kriebel ${ }^{17}$ compared the measurements of Isaacson with three theoretical models for predicting wave transmission through a vertical wave barrier. The experimental data of Isaacson matched the calculations of Kriebel very well. Kriebel performed additional model testing. Once again it was proved that larger screen drafts contributed positive to the transmission coefficient.

\subsection{Conclusions}

Although a lot of theoretical and practical research has been done, no practical solution has been found for the general problem of creating a floating breakwater, able to attenuate waves of a wide frequency range. The floating breakwaters that have been built in real situations were designed to serve at specific locations with specific wave conditions.

This resulted in huge and expensive constructions, like the Monaco floating breakwater, or cheap and temporary structures like the RIBS floating breakwater.

From the model tests, as they are described in paragraph 3.4 and appendix A, it can be concluded that there seems to be an optimal floating breakwater design for every wave frequency. The structural appearance of the floating breakwater might change whenever the frequency of the incoming wave train changes in order to attenuate the wave optimal.

Striking is the fact that one common conclusion returns in every study on floating breakwaters:

"A floating breakwater is able to attenuate waves to an acceptable level in a limited wave frequency range."

Although it seems to be rather obvious, this conclusion is never accompanied with the remark that a floating breakwater, able to change its appearance is expected to perform better on a wider wave spectrum. Or what Evans and Linton described as 'tuning the natural frequency' of the floating breakwater system. A floating breakwater that is able to change its dynamic behaviour on the present wave conditions can be the solution for many problems, encountered by large scale floating harbour terminals. However, one has to keep in mind that in real ocean conditions multiple wave frequencies will be present at each time step.

\footnotetext{
${ }^{16}$ Gardner, J.D., Townend, I.H. and Fleming, C.A. (1986). 'The design of a slotted vertical screen breakwater'. ASCE $20^{\text {th }}$ Coastal Engineering Conference.

${ }^{17}$ Kriebel, D.L. (1992). 'Vertical wave barriers. Wave transmission and wave forces'. ASCE $23^{\text {rd }}$ International Conference on Coastal Engineering.
} 


\section{Chapter 4}

\section{Problem definition \& Objectives}

\subsection{General}

A floating breakwater is not a real breakwater.

In fact, this simple but on the other hand complex statement contains the whole floating breakwater problem. A conventional, fixed breakwater reflects and absorbs the wave energy in order to create a sheltered area behind it. A floating 'breakwater' on the other hand, reflects and generates wave energy in order to obtain the same results as the fixed breakwater.

In other words: the fixed breakwater tries to diminish the energy of the incoming wave, while the floating breakwater uses this same incoming wave to generate anti-waves.

The model tests, discussed in chapter 3, prove that a floating breakwater is able to attenuate waves successfully in a limited frequency range. Outside this frequency range, the system is not this effective anymore. The problems that have to be solved and the objectives of this research are discussed in this chapter. The methodology of the design process will be presented at the end of this chapter.

\subsection{Problem definition}

The development of transportable, floating harbours is important to increase the efficiency of the container transhipment business. Several floating transhipment container terminals at strategic places in the ocean will decrease the amount of miles a mega vessel has to travel to reach a fixed harbour deep enough to receive it. On the other hand, harbour efficiency is also measured by the amount of 'down-time' of the harbour. This is the time, in weeks per year, in which a harbour cannot be used due to extreme conditions. One can imagine that the down-time risk is much higher when open-ocean conditions are considered.

The main problem of this whole thesis is how to create an area where harbour activities can take place in deep, unprotected water conditions. 


\subsection{Objectives}

A floating structure has to be developed that is capable of attenuating the incoming waves to such a level that a floating harbour can become an efficient alternative.

The dynamic behaviour of floating structures in waves depends on a lot of factors. It is important to understand the effect of the hydrodynamic as well as the structural factors that are involved. With this knowledge, a model can be created to state the optimal structural dimensions at several wave conditions. The model can be used as a design tool to determine a theoretical-based design. The main objective of this master's thesis is therefore twofold:

- Determine the influence of the several structural elements on the dynamic behaviour of the floating breakwater and create a model that proves the influence of these elements.

- $\quad$ Create a constructional design, based on this model to prove that a floating breakwater is possible from a constructive point of view.

It is crucial to realise the importance of the several (hydro)dynamic calculation parameters. For further design purposes it is very important to give advice on how to deal with the factors that have been assumed.

\subsection{The Input - Output model}

For the following chapters, an input-output model is used to reach the objectives as they were discussed in the previous paragraph.

The input of the ReFBreak model will be discussed in chapter 5 . The input is split in three areas of interest which determine the requirements and the boundary conditions of the model. The input has to be bounded very carefully since the ReFBreak model will become too complicated to use when too many factors are involved. Requirements and assumptions are explained in the input phase too.

Based on the information of chapter 5, the ReFBreak will be presented in chapter 6

The calculations will be checked with the numerical three-dimensional calculation program DELFRAC. A sensitivity analysis will be performed in order to state the reliability of the calculations and the importance of the assumptions on the hydrodynamic parameters that were done in chapter 6 .

With the ReFBreak-model, optimal floating breakwater dimensions can be determined. The optimal floating breakwater dimensions from a theoretical point of view will be discussed in chapter 7 . The influence of the structural variables on the hydrodynamic behaviour as well as the attenuating capacity will be discussed in a parameter analysis.

On the next page, a frame work is presented with all the factor that are involved in the ReFBreak model. The relations of the parameters and requirements and their influence on the wave transmission is presented in figure $4-2$. 


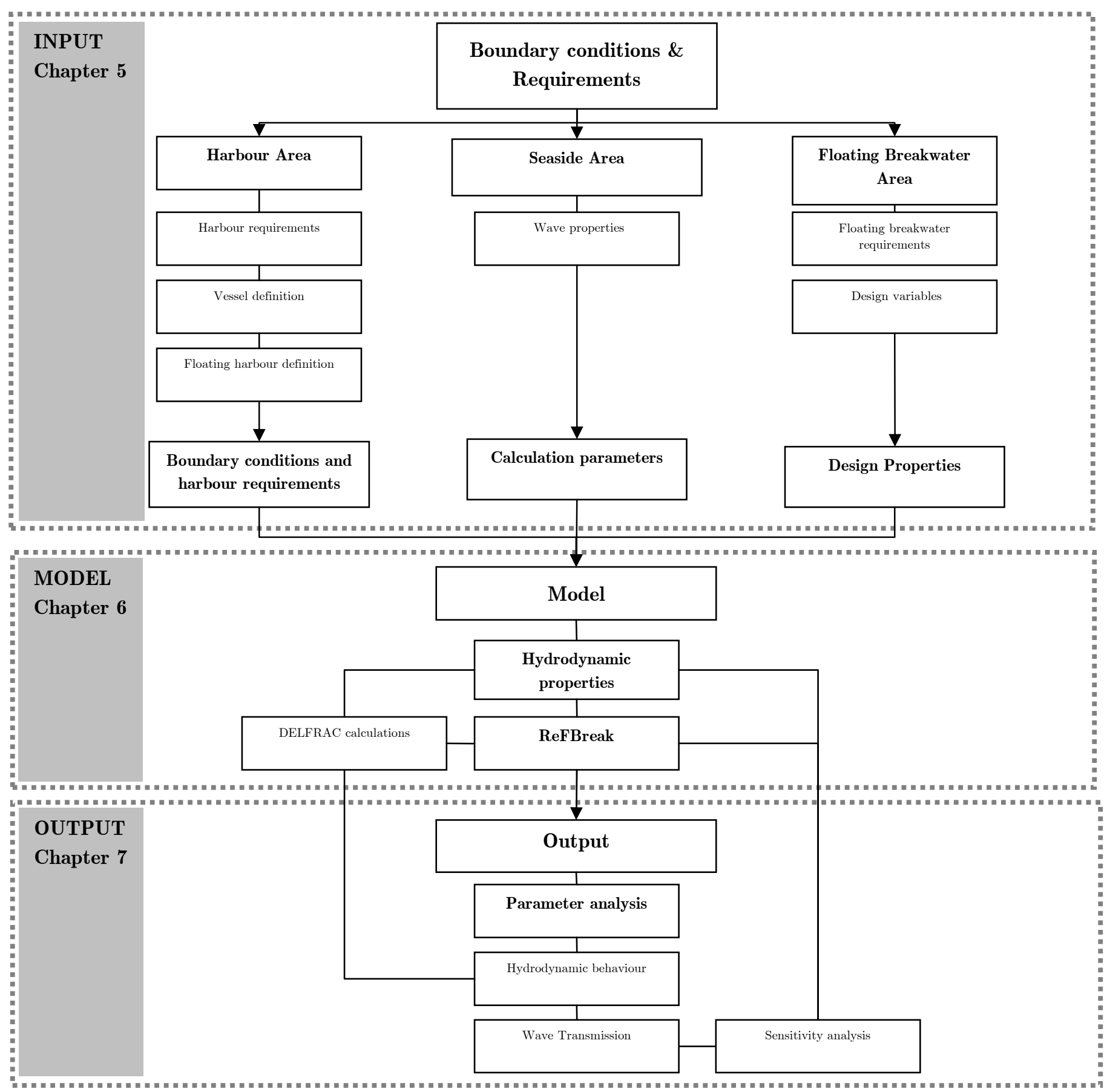

Figure 4-1: Framework of the ReFBreak-model 


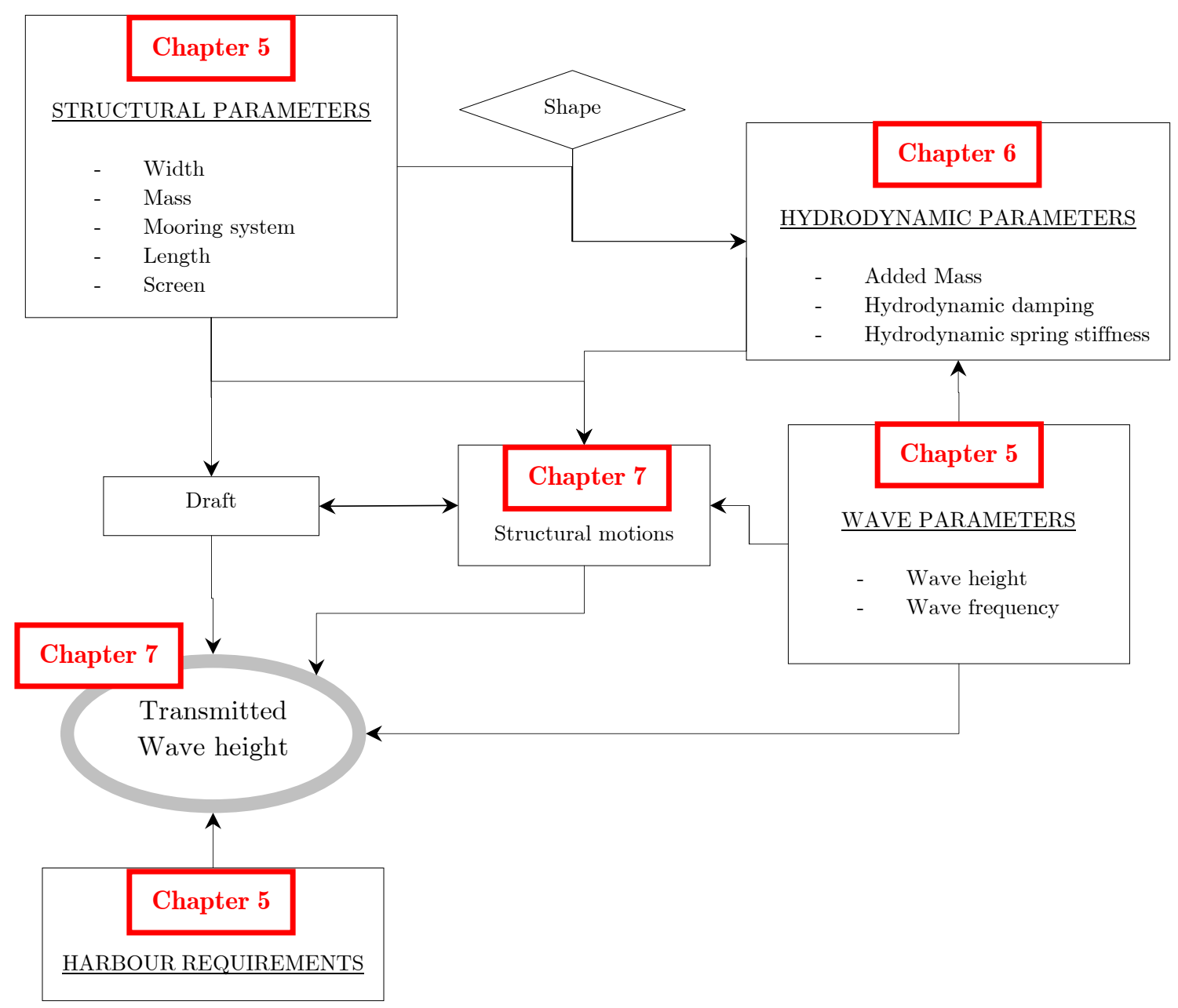

Figure 4-2: Factors and relations in the wave transmission calculation process 


\section{Chapter 5}

\section{Boundary conditions \& Requirements}

\subsection{General}

The boundary conditions, requirements and the structural assumptions are discussed in this chapter. Due to the complexity of the hydrodynamic floating harbour system, this chapter is sub divided into three major sections.

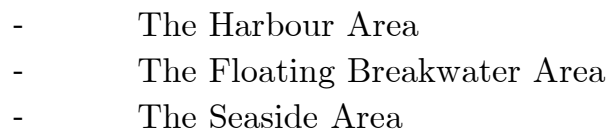

The harbour area will provide requirements on the operability of the FTCT. The mutual motions of FTCT and moored vessels are crucial from this perspective.

The seaside area on the other hand will provide the waves that have to be attenuated by the floating breakwater in order to satisfy the demands of the floating harbour. Type of waves and assumed wave conditions are discussed in paragraph 5.4.

The design properties of the floating breakwater itself will be discussed in paragraph 5.3. The floating breakwater has to meet certain requirements in order to be a solution for the container business, as discussed in chapter 3.3 .

Together with the hydrodynamic properties as they will be presented in chapter 6 , this chapter provides the input data necessary for the ReFBreak model.

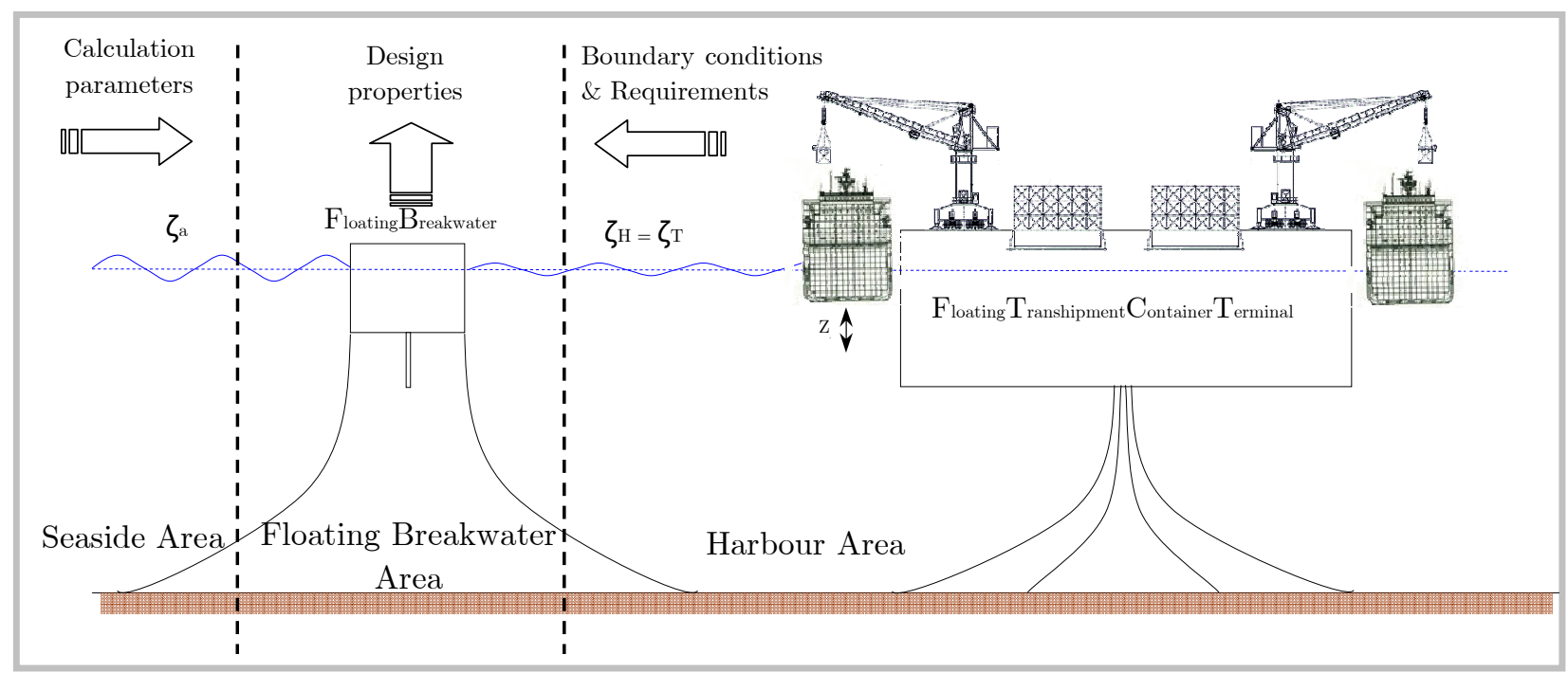

Figure 5-1: The floating harbour system. 


\subsection{Harbour Area}

\subsubsection{Harbour requirements}

A floating harbour can only become a success when a certain level of efficiency is guaranteed. A container harbour is expected to be operational during 50 weeks per year on average. Due to the complex dynamic behaviour of a floating harbour, such a level of efficiency will be hard to reach.

Nevertheless, the floating harbour is expected to be able to reach the same level of efficiency as a fixed harbour would in the same environmental conditions. The advantages of a floating harbour may not be diminished by a lower level of efficiency. It must be a valuable addition for the existing shipping business.

In this research, the focus will be on the container transhipment business since (un)loading of container vessels requires minimal motion amplitudes. The efficiency of the harbour deceases rapidly when the ship motions during harbour operations increase. This is shown in the table below.

\begin{tabular}{l|lcccccc}
\hline Ship type & Cargo handling equipment & $\begin{array}{c}\text { Surge } \\
(\mathrm{m})\end{array}$ & $\begin{array}{c}\text { Sway } \\
(\mathrm{m})\end{array}$ & $\begin{array}{c}\text { Heave } \\
(\mathrm{m})\end{array}$ & $\begin{array}{c}\text { Yaw } \\
\left({ }^{\circ}\right)\end{array}$ & $\begin{array}{c}\text { Pitch } \\
\left({ }^{\circ}\right)\end{array}$ & $\begin{array}{c}\text { Roll } \\
\left({ }^{\circ}\right)\end{array}$ \\
\hline Container vessels & $100 \%$ efficiency & 1.00 & 0.60 & 0.80 & 1 & 1 & 3 \\
& $50 \%$ efficiency & 2.00 & 1.20 & 1.20 & 1.5 & 2 \\
\hline
\end{tabular}

Table 5-1: Recommended ship motions during harbour operations. Motions refer to peak-peak values (except for sway: zero-peak) ${ }^{1}$

Since the smaller feeder vessels have their own unloading equipment, they do allow larger motions in practice. Mega vessels on the other are always depending on the unloading facilities of the harbour and are therefore more vulnerable to decreasing efficiency due to motions.

The combination of motion amplitudes and variations in time (period) are the cause of accelerations and roll. These accelerations do harm the working conditions on board of the ships and the floating harbour. Accelerations determine the loads on cargo and the equipment and are the main reason for seasickness. The limiting criteria of the operability for ships are defined by the Nordic co-operative project 'sea keeping performance of ships' (NORDFORSK, 1987). From [Faltinsen, 1995]²:

\begin{tabular}{l|l}
\hline & Merchant ships \\
\hline Vertical acceleration & $0.275 \mathrm{~g}$ \\
\hline Lateral acceleration & $0.120 \mathrm{~g}$ \\
\hline Roll (RMS-value) & 6.0 \\
\hline Table 5-2: General operability limiting criteria for ships (NORDFORSK, 1987)
\end{tabular}

${ }^{1}$ PIANC working group no.24. (1995). 'Criteria for movements of moored ships in harbours, A practical guide'.

${ }^{2}$ Faltinsen, O.M. (1995) 'Sea loads on ships and offshore structures'. Cambridge University Press. 


\subsubsection{Design vessel}

The container handling business increased with an incredible rate the past 30 years. This is mainly due to the increase of efficiency and the introduction of mega vessels. The need for deepwater container terminals to allow further growth of the business was already clarified in chapter 2.4. Floating Transhipment Container Terminals (FTCT) are visited by large mega vessels as well as by the smaller feeder vessels. Every type of ship that visits the FTCT demands a different maximum wave condition in which it can be served with $100 \%$ efficiency.

Examples of ship types that can be expected to be served by the FTCT are listed in the table below.

\begin{tabular}{|l|l|c|c|c|c|c|c|c|c|}
\hline Ship size & TEU & $\mathbf{4 0 0}$ & $\mathbf{6 0 0}$ & $\mathbf{8 0 0}$ & $\mathbf{1 , 0 0 0}$ & $\mathbf{1 , 2 0 0}$ & $\mathbf{1 , 5 0 0}$ & $\mathbf{2 , 0 0 0}$ & $\mathbf{2 , 5 0 0}$ \\
\hline Container ship class & & Small & Small & Small & Small & Feeder & Feeder & Feeder & Feeder \\
\hline Deadweight (design) & $\mathrm{dwt}$ & 5,700 & 8,400 & 11,000 & 13,500 & 16,000 & 20,000 & 26,000 & 31,000 \\
\hline Length overall & $\mathrm{m}$ & 105 & 122 & 136 & 150 & 155 & 175 & 185 & 200 \\
\hline Breadth & $\mathrm{m}$ & 17.2 & 19.2 & 21.4 & 22.7 & 25.0 & 28.0 & 30.0 & 30.0 \\
\hline Design draught & $\mathrm{m}$ & 6.5 & 7.6 & 8.2 & 8.7 & 9.2 & 10.0 & 11.5 & 11.5 \\
\hline
\end{tabular}

\begin{tabular}{|l|c|c|c|c|c|c|c|c|c|}
\hline Ship size & TEU & $\mathbf{3 , 0 0 0}$ & $\mathbf{4 , 0 0 0}$ & $\mathbf{4 , 5 0 0}$ & $\mathbf{5 , 0 0 0}$ & $\mathbf{6 , 0 0 0}$ & $\mathbf{8 , 0 0 0}$ & $\mathbf{1 2 , 0 0 0}$ & $\mathbf{1 8 , 0 0 0}$ \\
\hline Container ship class & & Panamax & Panamax & Panamax & Post-Panamax & Post-Panamax & Post-Panamax & Suezmax & Post-Suezmax \\
\hline Deadweight (design) & $\mathrm{dwt}$ & 37,000 & 48,000 & 54,000 & 59,000 & 70,000 & 93,000 & 137,000 & 200,000 \\
\hline Length overall & $\mathrm{m}$ & 220 & 280 & 293 & 280 & 305 & 355 & 400 & 470 \\
\hline Breadth & $\mathrm{m}$ & 32.2 & 32.2 & 32.2 & 40.0 & 43.0 & 43.0 & 52.5 & 60.0 \\
\hline Design draught & $\mathrm{m}$ & 12.0 & 11.8 & 12.2 & 12.5 & 12.5 & 13.6 & 14.6 & 15.7 \\
\hline
\end{tabular}

Table 5-3: Container ship types

Small feeder container vessels are applied for short sea container transportation. The larger feeder vessels until 2,500 TEU (Twenty feet Equivalent Unit) are normally used to feed the mega container vessels. They are also applied in areas where the demand for large container vessels is too low.

Until 1988, the hull dimensions of the largest container ships, the so-called Panamax-size vessels, were limited by the length and breadth of the lock chambers of the Panama Canal. The Panama Canal allows vessels with a maximum beam of $32.3 \mathrm{~m}$ and a maximum length of $294.1 \mathrm{~m}$ to pass.

In 1988, a ship with a beam of more than $32.3 \mathrm{~m}$ was built. This ship was not able to pass the Panama Canal any more and is therefore called the Post-Panamax-size vessel.

Ultra Large Container Ships (ULCS), carrying as much as 16,000 TEU, may be expected in the near future. This ship type (Suez max.), with a beam of 50-57 $\mathrm{m}$ and a maximum draft of $16.4-14.4 \mathrm{~m}$ is the maximum ship size that can pass the Suez Canal.

Recent investigations ${ }^{3}$ indicate that in approximately 10 years, Ultra Large Container Vessels with a TEU of 18,000 , a beam of $60 \mathrm{~m}$ and a draft of $21 \mathrm{~m}$ can be expected. These vessels can be classified as Post-Suezmax, or Malacca-max container vessels.

\footnotetext{
${ }^{3}$ Wijnolst, N., Scholtens, M., Waals, F. (1999). 'Malacca-max: the ultimate container carrier'. Delft University Press
} 


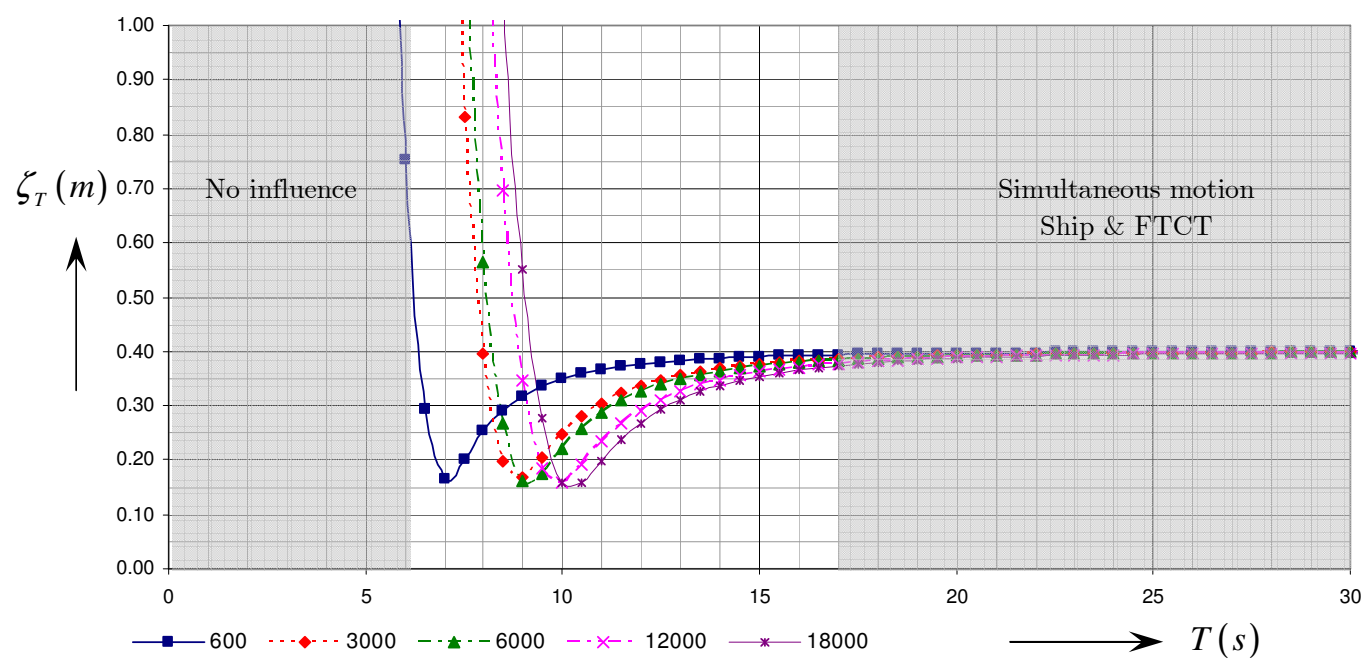

Figure 5-2: Maximum allowable wave amplitude ( $m$ ) for several ship sizes (TEU)

Every vessel in table 5-3 will react differently on the incoming waves. This is due to the different cross sectional layouts and weights. Figure 5-2 shows the maximum allowable wave amplitude per wave period inside the harbour for several ship sizes. It is clear that the smallest ship (600 TEU) is the governing vessel for wave periods smaller than $8.5 \mathrm{~s}$. Waves longer than 17 seconds will cause a simultaneous reaction of ship and FTCT. Waves with periods between 6.5 and 11 seconds are the ones that cause most damage to the harbour efficiency. The wave frequencies in this range are close to the natural frequencies of the vessels. The wave amplitude should not exceed 0.15 metres in this range. The graphs that were shown in figure 5-2 are obtained with equation 6.40. The equation is explained in Appendix B.

\subsubsection{Floating Transhipment Container Terminal}

A floating transshipment container terminal (FTCT) is a pontoon-shaped structure where container transshipment in offshore conditions takes place. A FTCT has the same facilities and capabilities as a modern container harbour:

- $\quad$ Loading/unloading of containers;

- $\quad$ Intern transport of containers;

- $\quad$ Storage of containers

- $\quad$ Processing vessels

A research on this topic was performed by $\mathrm{Ali}^{4}$ in order to find the optimal FTCT design. In his report, Ali concluded that the pontoon should have a rectangular layout with a length of $1190 \mathrm{~m}$, a width of $240 \mathrm{~m}$ and a draft of $10.30 \mathrm{~m}$. In order to maintain a beam-on position, relative to the waves during operating conditions, it was decided that the FTCT should have a DP-thrusters turret mooring system. This mooring system creates the possibility of rotating the FTCT in such a way that the working efficiency is not affected for wave amplitudes up to three meters.

${ }^{4}$ Ali, A. (2005). Msc thesis 'Floating Transshipment Container Terminal', Delft University of Technology. 


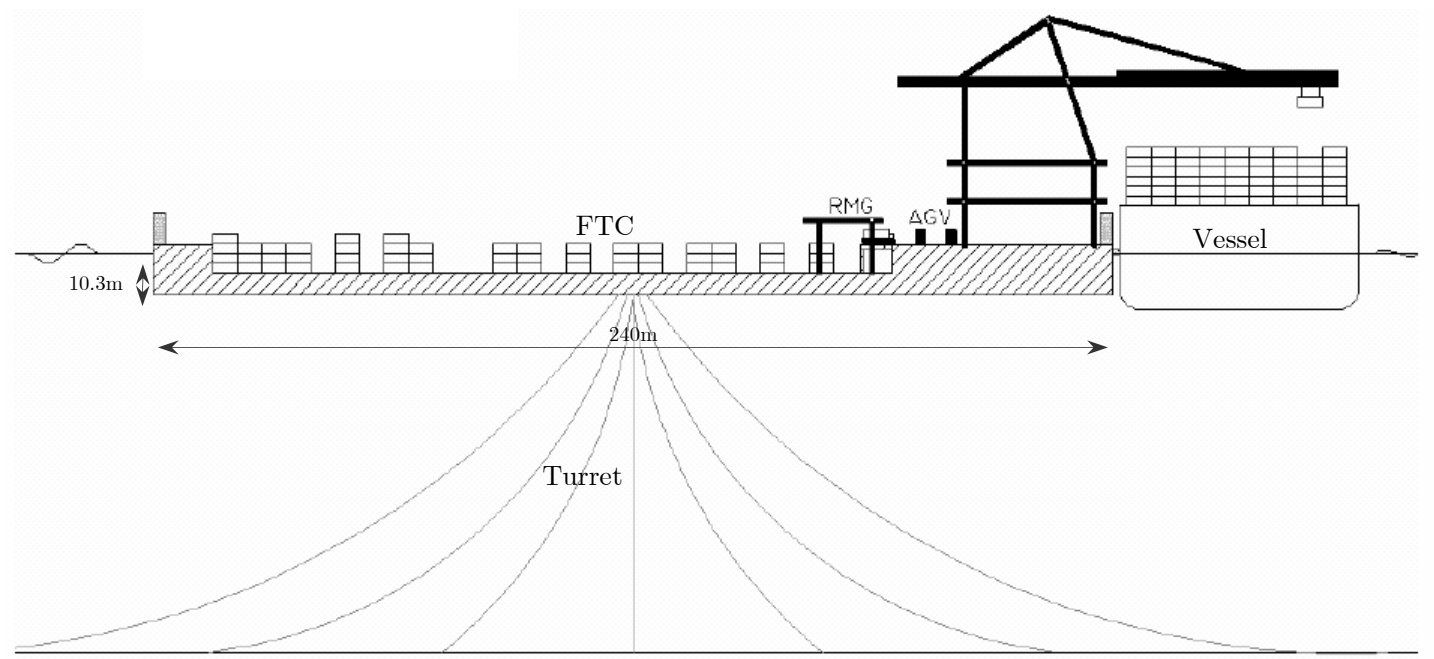

Figure 5-3: FTCT cross-section (from Ali $\left.(2005)^{5}\right)$.

Due to the mooring system, a situation where only beam waves occur is assumed for this research. The influence of the mooring system itself on the vertical dynamic behaviour of the FTCT is assumed to be negligible.

For the further research, the FTCT has a constant draft of $10.3 \mathrm{~m}$ and a width of $240 \mathrm{~m}$. Activities on the FTCT are assumed not to have any influence on the dynamic behaviour.

Few motions are encountered when the FTCT is forced by waves with relative short periods. The moored ship on the other hand will show large amplitude of motion when the same waves conditions are considered. Long wave periods will move the FTCT and the ship simultaneously. This is the reason why long waves will not affect the working efficiency of the FTCT. Figure 5-4 shows the effect of the wave periods on the mutual dynamic behaviour of the FTCT-vessel system.
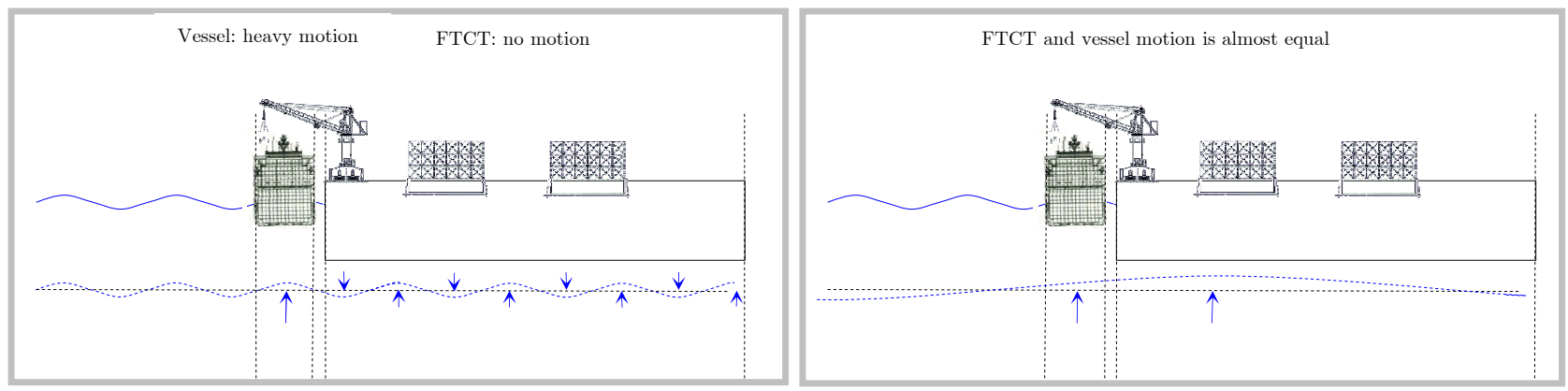

Figure 5-4: Mutual motion of vessel and FTCT

${ }^{5}$ Ali, A. (2005). Msc thesis 'Floating Transshipment Container Terminal', Delft University of Technology. 


\subsection{Floating Breakwater Area}

\subsubsection{Floating breakwater requirements}

The floating harbour will lose its efficiency when the wave conditions inside the harbour area reach a maximum. The maximum motions, allowed for both the vessel and the floating harbour, were discussed in paragraph 5.2. In open-sea conditions, the waves that do cause these problems, will often be to large and will affect the harbour efficiency for most of the time. A structure, especially designed to attenuate the harmful waves will be indispensable in this situation.

As was shown in figure 5-2, it is not necessary for the floating breakwater to be able to attenuate waves of the whole wave period range. Waves with periods that are between 6.5 and 11 seconds will cause most of the damage to harbour activities and should therefore be diminished.

Model tests proved the fact that floating breakwaters are able to attenuate waves in a limited period range (chapter 3). This limited performance is the main negative characteristic affecting the development of floating breakwaters in the past. Floating breakwaters can only be applied in areas with relative moderate wave conditions. The floating breakwaters that have been built were designed for specific locations, where specific wave conditions were expected.

A new kind of floating breakwater should discern itself by being applicable in a wide wave spectrum. With this characteristic, it will be possible to create floating harbours in open-sea conditions and to reuse them at several locations in the world.

From an economic and a technical point of view, the floating breakwater should be maintainable and transportable. Since the floating harbour is a dynamic system, it has to be possible to rearrange the position of the floating breakwater.

Poor soil conditions are often an argument to apply a floating structure instead of a fixed one. Because of this, the mooring of the floating breakwater must put minimum demands on the local soil conditions.

Summarized, the main requirements that a floating breakwater has to fulfil are:

1. Attenuate waves of the critical period range

2. Applicable on several locations in several conditions

3. Manageable, transportable

4. Reusable

5. Durable

The focus of the next chapters will be on the first two requirements. The numerical calculations will be performed in order to meet these requirements. Chapter 8 will concentrate on how to deal with requirements 3 to 5 . 


\subsubsection{Design variables}

The dynamic behaviour of a floating breakwater is influenced by many factors. The influence factors that can be distinguished are shown in figure 5-5 and can roughly be divided into three parts:

The interference of the floating breakwater with the environment

- $\quad$ The interference of the floating breakwater with the mooring system

- $\quad$ The interference of the floating breakwater with the other breakwater segments

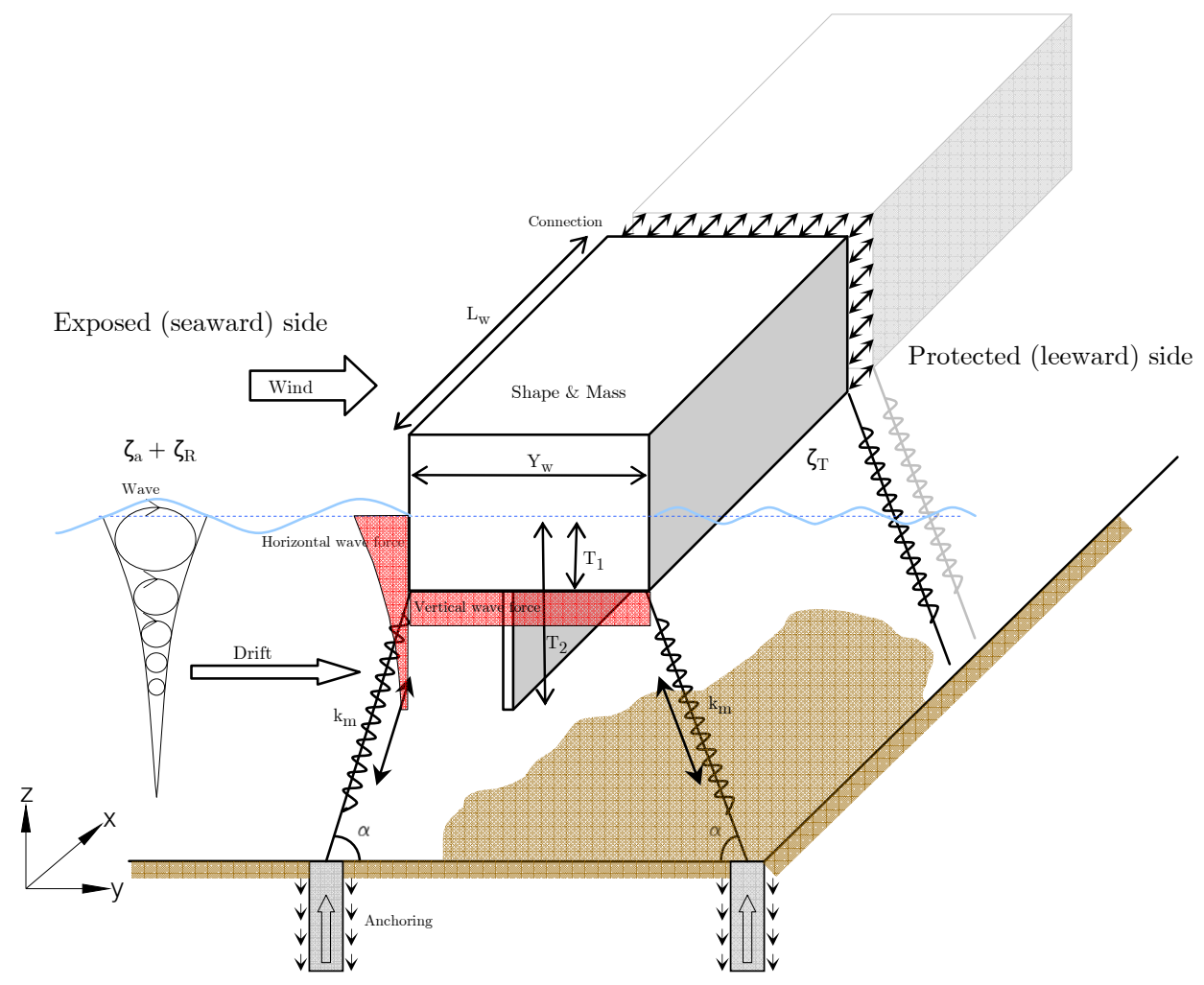

Figure 5-5: Structural and hydrodynamic influence factors.

The symbols, used in figure 5-5 are:

$\begin{array}{llll}k_{m} & : & \text { Spring stiffness of the mooring system } & (\mathrm{kg} / \mathrm{s}) \\ L_{w} & : & \text { Length of the floating breakwater element } & (\mathrm{m}) \\ T_{1} & : & \text { Draft of the floating section of the breakwater. } & (\mathrm{m}) \\ T_{2} & : & \text { Draft of the screen underneath the floating section of the breakwater } & (\mathrm{m}) \\ Y_{w} & : & \text { Width of the floating section of the floating breakwater } & (\mathrm{m}) \\ \zeta_{a} & : & \text { Incoming wave amplitude } & (\mathrm{m}) \\ \zeta_{R} & : & \text { Radiated / reflected wave amplitude to the sea side of the floating breakwater } & (\mathrm{m}) \\ \zeta_{T} & : & \text { Radiated / transmitted wave amplitude to the harbour side of the floating breakwater } & (\mathrm{m})\end{array}$

It is almost impossible to discuss all elements affecting the appearance or the performance of the floating breakwater. The design assumptions that have been made and the relevant structural elements that will be investigated are presented in this section. 


\section{The interference of the floating breakwater with the environment}

The local wave and wind conditions produce the forces that will put the floating breakwater into motion. Since the largest part of the floating breakwater will be submerged, the influence of the wave forces will be large. Wind forces will thus be neglected during the rest of this report.

The dynamic behaviour of a mass-spring system, like a floating body, is affected by the mass of the structure and the hydrodynamic parameters. The magnitude of the hydrodynamic parameters, as they will be discussed in the next chapter, depends on the frequency of motion and the structural dimensions. The shape and the dimensions do affect the structural behaviour and are the factors that determine the performance of the floating breakwater.

The scale-model test results, as they are discussed in appendix A, prove that the heave motion has a positive contribution on the performance of the floating breakwater. In addition, it can be stated that a structure that performs less sway and roll motions will radiate less wave energy. Because of these arguments, the shape of the floating breakwater is taken rectangular for the rest of this thesis. A design study of the breakwater shape is out of the scope of this research.

The dimensions and the mass of the rectangular structure however are important parameters that can be used to optimize the floating breakwater performance. The influences of the following structural dimensions are investigated during the numerical calculations:

$\begin{array}{lll}- & \text { Structural width (y-direction) } & \left(\mathrm{Y}_{\mathrm{w}}\right) \\ - & \text { Draft of the floating section (z-direction) } & \left(\mathrm{T}_{1}\right) \\ \text { - } & \text { Mass of the structure } & (\mathrm{m}) \\ \text { - } & \text { Draft of the screen underneath the structure } & \left(\mathrm{T}_{2}\right) \\ \text { - } & \text { Length of the structure (x-direction) } & \left(\mathrm{L}_{\mathrm{w}}\right)\end{array}$

\section{The interference of the floating breakwater with the mooring system}

The anchoring of the floating breakwater is necessary to keep the structure at the position where it is supposed to be. Besides the station keeping property, the mooring system is an important parameter that determines the dynamic behaviour of the mass-spring system.

The vertical motion can have a positive influence on the attenuating capacity of the floating breakwater. It is therefore important to realize that a stiff mooring system can affect this positive contribution in a negative way. Besides these dynamic restrictions, there are also the structural requirements 2 and 4 in section 5.3 .1 that prefer a mooring system, applicable at several locations regardless of the local soil conditions.

For the coming chapters, a catenary mooring system is used which has only limited influence on the short-term dynamic behaviour of the floating breakwater. The effect of tides on the short-term properties of the mooring lines, as it is shown in figure 5-6, is not considered in this research. The mooring lines are assumed to hold the structure at its position when long waves are considered.

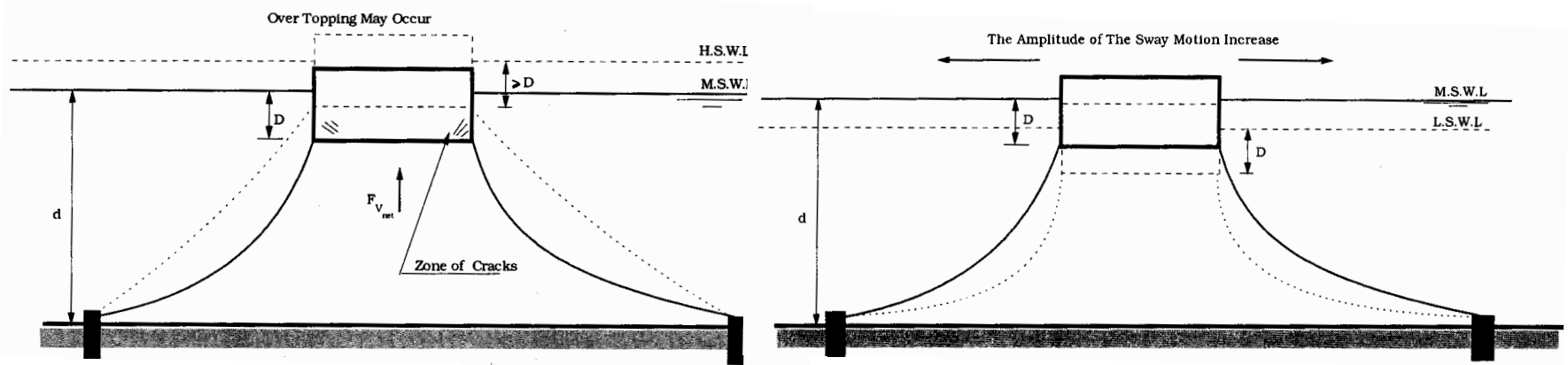

Figure 5-6: Tidal influence on the mooring line influence. ${ }^{6}$

${ }^{6}$ Tolba, E.R.A.S. (1998). 'Behaviour of floating breakwaters under wave action', PhD thesis, Suez Canal University of Egypt. 


\section{The mutual interference of the floating breakwater segments}

The total floating breakwater system consists of several mutually connected barges. Oblique and irregular incoming waves will move the individual floating breakwater sections differently. Due to the connections, the floating breakwater sections will influence each other in all degrees of freedom.

The mutual interaction of the floating breakwater sections is neglected in this report since beam-waves will be used in the numerical model. With this assumption, all the floating breakwater sections will move simultaneously and no mutual difference will occur. The connection between the floating breakwater segments is a study itself. It is hard to estimate the influence of a connection in this stage of the research. 


\section{$5.4 \quad$ Seaside Area}

\subsubsection{General}

To understand the forces and motions a floating breakwater will encounter, this chapter will create insight in the hydrodynamic interaction with the environment where it is located.

The floating breakwater will be applied in deep water where it should attenuate the present deep water waves. The main property of deep water- or short waves is the limited energy penetration into the depth. The wave energy of a deep water wave is not able to reach the bottom. In other words: the bottom will have no influence on the behaviour of the wave. When the water depth is more than $1 / 2$ times the wave length $(h / \lambda>1 / 2)$, a wave is called a deepwater wave.

In practice, waves can be discerned in four categories:

1) Waves generated by vessels or other water-moving objects

2) Waves generated by the interaction of the water surface with the wind

3) Waves generated by astronomical forces: tides

4) Waves generated by geomorphologic activities: Tsunamis

The main objective during this research is to attenuate waves of the second category. These waves do harm the harbour activities because of their period as well as their amplitudes.

Waves of the first category are insuperable since these waves are also created inside the harbour area. Little influence on harbour activities is created by waves of category three. Since these waves have very long periods, floating harbour as well as moored vessel are able to follow the slowly varying movements. However, to calculate the (static) forces on the mooring lines, these waves can not be neglected.

Waves of category four are neglected during this research since these waves rarely occur and the impact of these waves in deep water conditions is hardly noticeable.

In this chapter, the properties of regular deep water waves are discussed. 


\subsubsection{Wind generated waves}

The wind waves can be distinguished into two classes, related to their origination: sea waves and swell.

A sea is a train of waves driven by the local wind field. They are characterized by the relative short and steep crests, compared to their wave lengths. Sea waves have a rather irregular character. High crested and low crested waves are following each other in an unpredictable way. Also the directions of the waves can vary with tens of degrees.

Swell are waves that are created by the wind in an area at some distance from the considered area. The crests of these waves are flattened out and the wave profile is more regular than sea waves.

In normal circumstances a combination of the sea and swell waves exists at any given time. Therefore, the natural sea surface has to be modelled as an irregular wave pattern.

Sea in general has higher wave amplitudes and shorter wave periods than swell. In the offshore world, wave spectra are used to determine the incoming wave energy. Three methods to obtain a wavespectrum are used, which are:

- The Pierson-Moskowitz wave spectrum. The spectral density is obtained by linking the significant wave height and the wave period with a fixed relation.

- The Bretschneider wave spectrum. This is also known as the modified two-parameter Pierson-Moskowitz wave spectrum since the spectral density is a function of the significant wave height and the mean centroid wave period.

- $\quad$ The JONSWAP (Joint North Sea Wave Project) wave spectrum. The spectral density is based upon measurements that were carried out along a line extending over 100 miles into the North Sea.

The position of the considered location determines which wave spectrum has to be used. The Pierson-Moskowitz spectrum is used when fully developed seas are considered. When open ocean-conditions are to be expected, the Bretschneider wave spectrum has to be used. The JONSWAP spectrum is especially suitable for near shore locations. Since the floating harbour will not be too far away from the mainland, the JONSWAP spectrum is expected to give the most realistic representation.

The measurements that were performed to obtain the JONSWAP spectrum were also used to link the wave periods and amplitudes to the Beaufort scale. Table 5-4 shows this relation. From this table it is clear that waves in near-shore areas with periods longer than $10[\mathrm{~s}]$ are certainly always swell.

\begin{tabular}{|c|c|c|c|c|c|c|c|c|}
\hline \multicolumn{9}{|c|}{ Wave Spectrum Parameter Estimates } \\
\hline \multirow[t]{3}{*}{$\begin{array}{l}\text { Scale of } \\
\text { Beaufort }\end{array}$} & \multirow{3}{*}{$\begin{array}{c}\text { Wind speed } \\
\text { at } 19.5 \mathrm{~m} \\
\text { above sea } \\
(\mathrm{kn})\end{array}$} & \multicolumn{3}{|c|}{$\begin{array}{c}\text { Open Ocean Areas } \\
\text { (Bretschneider) }\end{array}$} & \multicolumn{4}{|c|}{$\begin{array}{l}\text { North Sea Areas } \\
\text { (JONSWAP) }\end{array}$} \\
\hline & & $\mathrm{H}_{1 / 3}$ & $\mathrm{~T}_{1}$ & $\mathrm{~T}_{2}$ & $\mathrm{H}_{1 / 3}$ & $\mathrm{~T}_{1}$ & $\mathrm{~T}_{2}$ & $\gamma$ \\
\hline & & $(\mathrm{m})$ & $(\mathrm{s})$ & $(\mathrm{s})$ & $(\mathrm{m})$ & $(\mathrm{s})$ & $(\mathrm{s})$ & $(-)$ \\
\hline 1 & 2.0 & 1.10 & 5.80 & 5.35 & 0.50 & 3.50 & 3.25 & 3.3 \\
\hline 2 & 5.0 & 1.20 & 5.90 & 5.45 & 0.65 & 3.80 & 3.55 & 3.3 \\
\hline 3 & 8.5 & 1.40 & 6.00 & 5.55 & 0.80 & 4.20 & 3.90 & 3.3 \\
\hline 4 & 13.5 & 1.70 & 6.10 & 5.60 & 1.10 & 4.60 & 4.30 & 3.3 \\
\hline 5 & 19.0 & 2.15 & 6.50 & 6.00 & 1.65 & 5.10 & 4.75 & 3.3 \\
\hline 6 & 24.5 & 2.90 & 7.20 & 6.65 & 2.50 & 5.70 & 5.30 & 3.3 \\
\hline 7 & 30.5 & 3.75 & 7.80 & 7.20 & 3.60 & 6.70 & 6.25 & 3.3 \\
\hline 8 & 37.0 & 4.90 & 8.40 & 7.75 & 4.85 & 7.90 & 7.35 & 3.3 \\
\hline 9 & 44.0 & 6.10 & 9.00 & 8.30 & 6.10 & 8.80 & 8.20 & 3.3 \\
\hline 10 & 51.5 & 7.45 & 9.60 & 8.80 & 7.45 & 9.50 & 8.85 & 3.3 \\
\hline 11 & 59.5 & 8.70 & 10.10 & 9.30 & 8.70 & 10.00 & 9.30 & 3.3 \\
\hline 12 & $>64.0$ & 10.25 & 10.50 & 9.65 & 10.25 & 10.50 & 9.80 & 3.3 \\
\hline
\end{tabular}

Table 5-4: Wave conditions in open ocean areas and North Sea areas".

${ }^{7}$ Journée, J.M.J. and Massie, W.W. 'Offshore Hydromechanics' $1^{\text {st }}$ edition, Delft University of Technology. 
The factors, used in table 5-4 represent:

$\mathrm{H}_{1 / 3} \quad: \quad$ Significant wave height

$\mathrm{T}_{1} \quad$ : $\quad$ Mean centroid wave period

$\mathrm{T}_{2} \quad: \quad$ Mean zero-crossing wave period

$\gamma \quad: \quad$ peakedness factor

The floating breakwater has to attenuate waves with an irregular character. Irregular waves are in fact a superposition of several regular waves with varying directions and phase shifts. This superposition is shown in figure 5-7. It is important to understand the properties of these regular waves in order to be able to model the ocean surface.

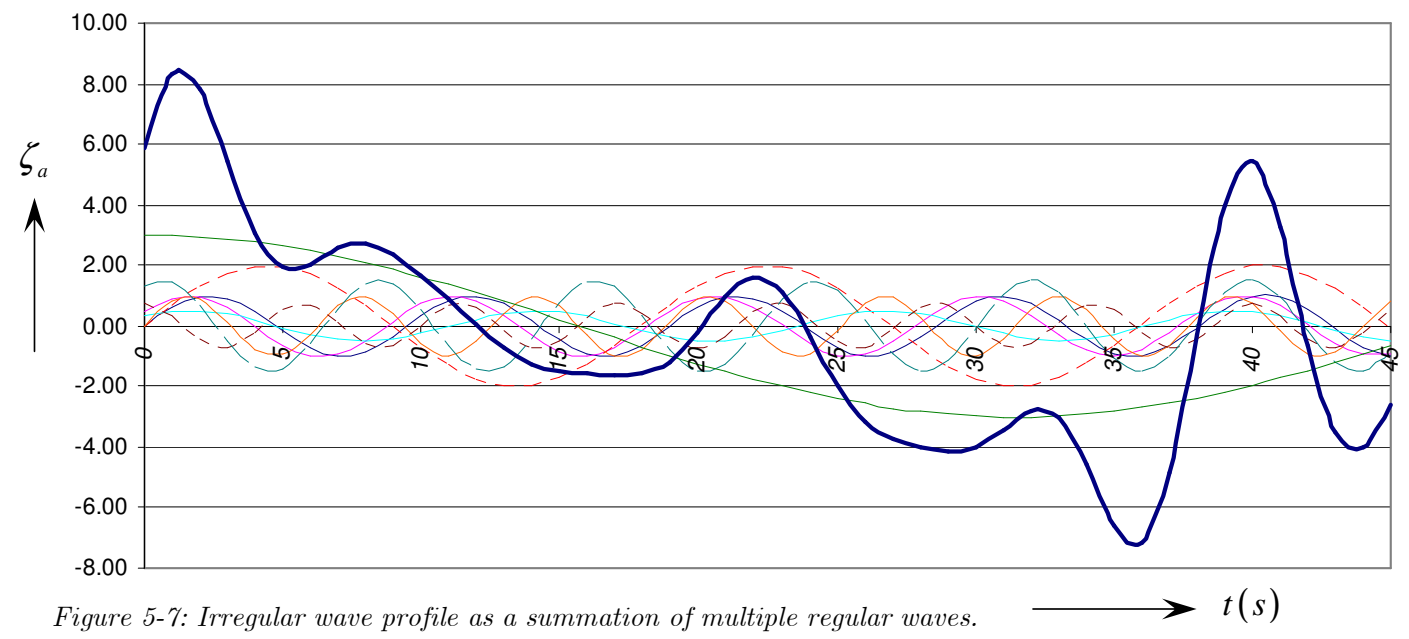

Despite the fact that waves in open-ocean conditions differ from regular waves, regular waves will be used in this theoretical research to state the performance of the floating breakwater. Reasons for this simplification are:

1) Regular waves do have one specific wave period $T[\mathrm{~s}]$. Irregular waves on the other hand can have more than one wave periods at the same time. To determine the optimal breakwater dimensions, it is essential to know the dynamic behaviour of the floating breakwater in a wide range of wave periods. This is only possible when the specific wave periods can be treated separately.

2) A prevailing wave period can often be distinguished in ocean-conditions. The performance of the floating breakwater will be optimal when the floating breakwater is able to attenuate the waves in a range around this peak-period. Knowledge about the performance of the floating breakwater at unique wave periods is a prerequisite to obtain an optimal breakwater design. 


\subsubsection{Regular waves}

A regular wave is a sinus-shaped wave with amplitude $\zeta_{a}[\mathrm{~m}]$ and a wave height $H=2 \zeta_{a}[\mathrm{~m}]$. The wave length is from now on called $\lambda[\mathrm{m}]$ which is the distance between two successive wave crests. The time needed to see one wave length pass in a certain fixed point is called the wave period $\mathrm{T}[\mathrm{s}]$.

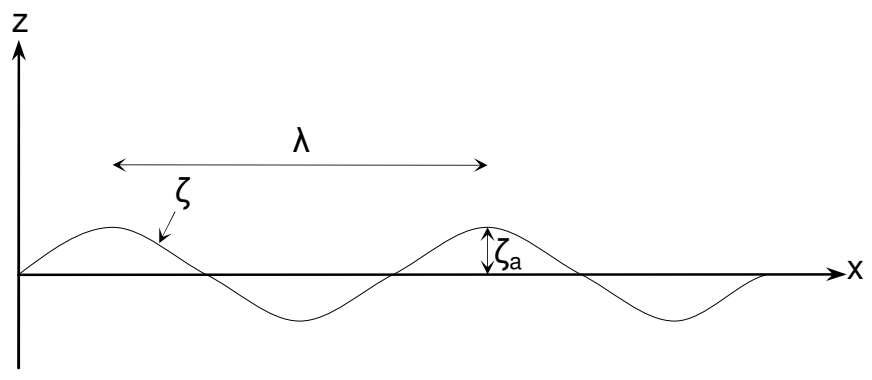

Figure 5-8: Wave parameters.

Since the regular wave pattern has a sinusoidal pattern, the wave length and period are converted to angles:

$\begin{array}{ll}\text { Wave number: } & k=\frac{2 \pi}{\lambda}[\mathrm{rad} / \mathrm{m}] \\ \text { Wave frequency: } & \omega=\frac{2 \pi}{T}[\mathrm{rad} / \mathrm{s}]\end{array}$

Where the wave length for deep water is given $\operatorname{as}^{8}$ :

$$
\lambda=\frac{g T^{2}}{2 \pi}
$$

In deep-water, the phase speed is denoted as:

$$
c=\frac{\lambda}{T}=\frac{\omega}{k}
$$

A combination of equations 6.3 and 6.4 yields the relation between the wave number and the wave frequency, the so-called dispersion relation:

$$
\omega^{2}=k g
$$

The surface elevation of the water in time as well as space can be described by the following equations:

Positive direction: $\quad \zeta=\zeta_{a} \cos (k x-\omega t)$

Negative direction: $\quad \zeta=\zeta_{a} \cos (k x+\omega t)$

The numerical calculations and the design study in this report are based on regular, sinus-shaped waves. This is done in order to be able to determine the optimal floating breakwater dimensions at different wave periods.

${ }^{8}$ Prof.dr.ir.J.A.Battjes, 'Short waves', Delft University of Technology. 


\subsection{Conclusions}

The floating breakwater, as it is considered in this report, has a rectangular shape with a screen underneath it. Structural dimensions, mass and the effect of a screen underneath the floating section are to be investigated with numerical calculations.

The influence of the mooring system on the dynamic behaviour of the floating breakwater is neglected in this report. The same holds for the dynamic influence of the individual floating breakwater sections in respect of each other.

The performance of the floating breakwater is directly related to the requirements defined by the harbour area. The floating breakwater must be able to increase the efficiency of the floating harbour. This efficiency is linked to the maximum allowed vertical motions of the vessels that are moored at the FTCT.

In this report regular, sinus-shaped waves will be considered to determine the hydrodynamic behaviour and the performance of the floating breakwater. With the potential theory, the kinematics and energy distribution in regular deep-water waves is discussed in Appendix B.II.

Since beam waves are considered for the rest of this report, only sway, heave and roll are the important degrees of freedom in which the floating breakwater is expected to move.

Although a floating body seems to be similar to a mechanical mass-spring system, this is not entirely true. The motions of the floating breakwater are affected by the hydrodynamic parameters like the added mass, damping and the hydrodynamic spring term. The magnitude of these parameters depends on the frequency of motion as well as the structural dimensions of the floating breakwater.

The dynamic behaviour of the floating breakwater can be changed by adjusting the (added)mass, the (hydrodynamic)damping or the (hydrodynamic)spring stiffness. The influences of the parameters and the mutual relations have to be investigated in chapter 7 of this report in order to state the optimal floating breakwater dimensions at a wide wave frequency spectrum. 


\section{Chapter 6}

\section{Theoretical Rectangular Floating Breakwater}

\section{Model ReFBreak}

In this chapter, a calculation model is presented that will serve as a design tool to state the optimal floating breakwater dimensions. The relations of the structural parameters, the hydrodynamic parameters and the wave parameters were shown in figure 4-2. The calculation model will show the effect of several constructional adaptations on the performance of the floating breakwater.

The calculation model as it is presented in this chapter will give pure theoretical solutions. As stated in chapter 5, regular beam waves are not representative for ocean conditions. The objective of the calculation model is to understand the behaviour of the floating breakwater when specific wave frequencies are considered.

A detailed description of the calculation model and the derivatives of the equations is given in Appendix B.

\subsection{Dynamic behaviour}

Considered that the floating breakwater will not produce any waves by itself due to structural movements when floating in still water, the only hydro mechanical reaction will occur after the structure is loaded with wave exciting forces and moments.

The wave exciting forces and motions will put the floating breakwater in a harmonic oscillation. The oscillating floating breakwater will produce waves, the so-called radiation waves. Since two types of waves can be distinguished, two types of wave loadings can be distinguished as well:

- $\quad$ Hydro mechanical forces and moments.

These forces and moments are induced by the harmonic oscillations of the rigid body, moving in the undisturbed surface of the fluid.

- $\quad$ Wave exciting forces and moments.

These forces and moments are produced by waves coming in on the floating body.

The hydro mechanical forces $(\vec{F})$ and moments $(\vec{M})$ can be split into four separate parts:

$\vec{F}=\overrightarrow{F_{r}}+\overrightarrow{F_{w}}+\overrightarrow{F_{d}}+\overrightarrow{F_{s}}$

$\vec{M}=\overrightarrow{M_{r}}+\overrightarrow{M_{w}}+\overrightarrow{M_{d}}+\overrightarrow{M_{s}}$

In which:

$\overrightarrow{F_{r}}, \overrightarrow{M_{r}} \quad$ : $\quad$ Waves radiated from the oscillating body in still water;

$\frac{r}{F_{w}}, M_{w} \quad: \quad$ Approaching waves on the fixed body;

$\overrightarrow{F_{d}}, \frac{M_{w}}{M_{d}} \quad: \quad$ Diffracted waves of the fixed body;

$\overrightarrow{F_{s}}, \overrightarrow{M_{s}} \quad$ : Hydrostatic buoyancy in still water. 
Figure 6-1 shows the calculation cycly including the influencing factors between the structures. As mentioned in the previous chapter, the interference between the floating breakwater sections will be neglected in this report.

Although this does not give the optimal representation of reality, it will be sufficient to provide insight in the effects of several constructional measures on the wave attenuation capacity of the floating breakwater.

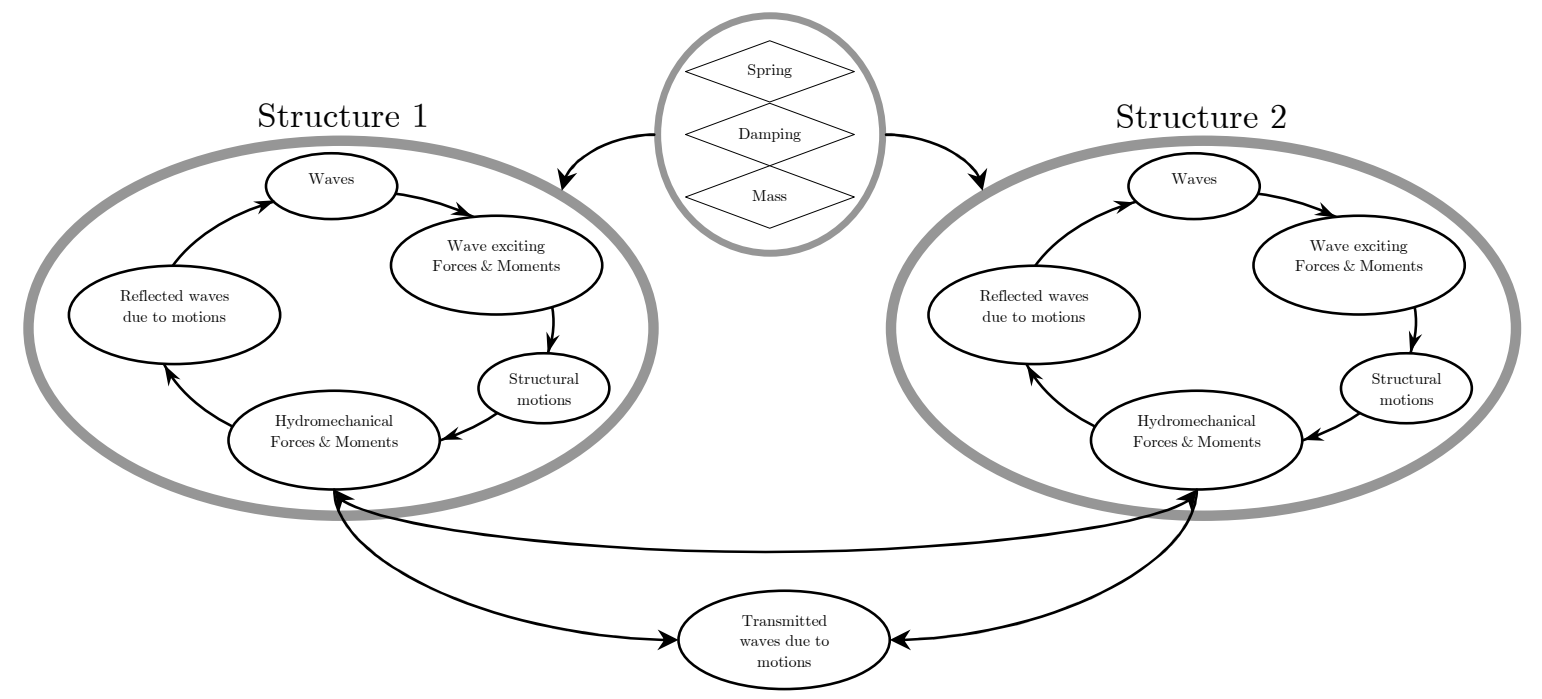

Figure 6-1: Calculation cycle for two connected floating breakwater sections. 


\subsubsection{Degrees of freedom}

For a floating body in a three-dimensional plane, 6 degrees of motions about the centre of gravity can be distinguished. These motions are shown in figure 6-2.

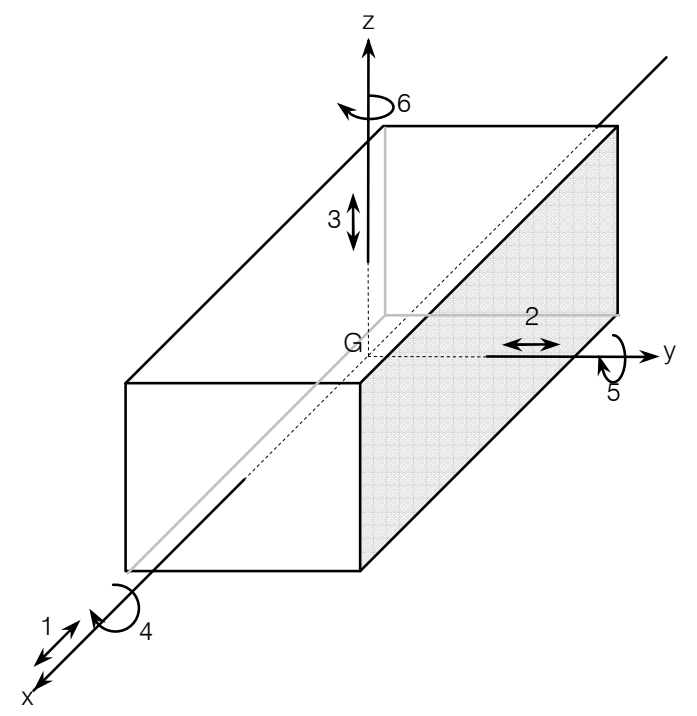

Figure 6-2: Degrees of freedom for a floating body in a three-dimensional space

Since the incoming waves put the structure into a harmonic oscillation, the equations of motion will represent a harmonic oscillation as well. The equations of motion for the 6 motions are:
1) Surge
2) Sway
$x=x_{a} \cos \left(\omega t+\varepsilon_{x \zeta}\right)$
3) Heave
$y=y_{a} \cos \left(\omega t+\varepsilon_{y \zeta}\right)$
4) Roll
$z=z_{a} \cos \left(\omega t+\varepsilon_{z \zeta}\right)$
$\phi=\phi_{a} \cos \left(\omega t+\varepsilon_{\phi \zeta}\right)$
5) Pitch
$\theta=\theta_{a} \cos \left(\omega t+\varepsilon_{\theta \zeta}\right)$
6) Yaw
$\psi=\psi_{a} \cos \left(\omega t+\varepsilon_{\psi \zeta}\right)$

$$
\}
$$

(6.1)

Where:

$\begin{array}{lll}\varepsilon_{n \zeta} & : & \text { Phase angle } \\ \omega=2 \pi / T & : & \text { Circular wave frequency }(\mathrm{rad} / \mathrm{s}) \\ n_{a} & : & \text { Motion amplitude }\end{array}$

The motions are not independent in respect to each other. The heave amplitude in a point on the two-dimensional yz-plane for example, is influenced by the magnitude of the pitch motion. This three-dimensional influence on the two-dimensional amplitudes exists, for the sway motions in the yz-plane too.

However, since beam waves are considered in this report, surge, pitch and yaw will not occur. The three-dimensional relation with the two-dimensional amplitudes is therefore omitted in this report. 


\subsubsection{The Hydrodynamic mass-spring system}

The floating breakwater will interact with the surrounding water. Extra conditions are necessary in order to be able to state the interference of the floating, oscillating body with the water particles. In Appendix B paragraph B.II.a, the four conditions concerning deep water boundary conditions were discussed. Three additional conditions can be added when an oscillating floating breakwater is involved.

5) Kinematic boundary condition on the oscillating body surface

6) Radiation condition

7) Symmetric or anti-symmetric conditions

Ad.5 The velocity of a water particle at a point at the surface of the body is equivalent to the velocity of this (watertight) body point itself.

$$
\frac{\delta \Phi}{\delta n}=v_{n}(x . y \cdot z ; t)=\sum_{j=1}^{6} v_{j} \cdot f_{j}(x, y, z)
$$

In which $\mathrm{f}$ is the direction cosine for the several degrees of freedom:
1) Surge : $\quad f_{1}=\cos (n, x)$
2) Sway : $\quad f_{2}=\cos (n, y)$
3) Heave : $f_{3}=\cos (n, z)$
4) Roll : $\quad f_{4}=y \cos (n, z)-z \cos (n, y)=y f_{3}-z f_{2}$
degrees of freedom in the $2 \mathrm{D}$ yz-plane
5) Pitch : $f_{5}=z \cos (n, x)-x \cos (n, z)=z f_{1}-x f_{3}$
6) Yaw : $\quad f_{6}=x \cos (n, y)-y \cos (n, x)=x f_{2}-y f_{1}$

The relation of the three rotational motions with the translational motions was clarified in the previous section. The direction cosines of the translational motions are used to obtain the direction cosines of the rotational motions.

Ad.6 The radiation condition states that as the distance, $\mathrm{R}$, from the oscillating body becomes large; the potential value tends to zero:

$\lim _{R \rightarrow \infty} \Phi=0$

Ad. 7 When the floating breakwater has a symmetric middle line, one can make use of this to simplify the potential equations:

$\begin{array}{lll}\text { Sway } & : & \Phi_{2}(-x, y)=-\Phi_{2}(+x, y) \\ \text { Heave }: & \Phi_{3}(-x, y)=+\Phi_{3}(+x, y) \\ \text { Roll }: & \Phi_{4}(-x, y)=-\Phi_{4}(+x, y)\end{array}$

Although a floating body seems to have the same dynamic characteristics as a mechanic mass-spring system, there is an important difference that affects the dynamic behaviour. The water, surrounding the oscillating floating breakwater will determine the total mass, the spring stiffness and the damping of the system. Since the magnitude of the so-called added mass and hydrodynamic damping parameters depend on the motion amplitude and frequency, these parameters are never constant. 


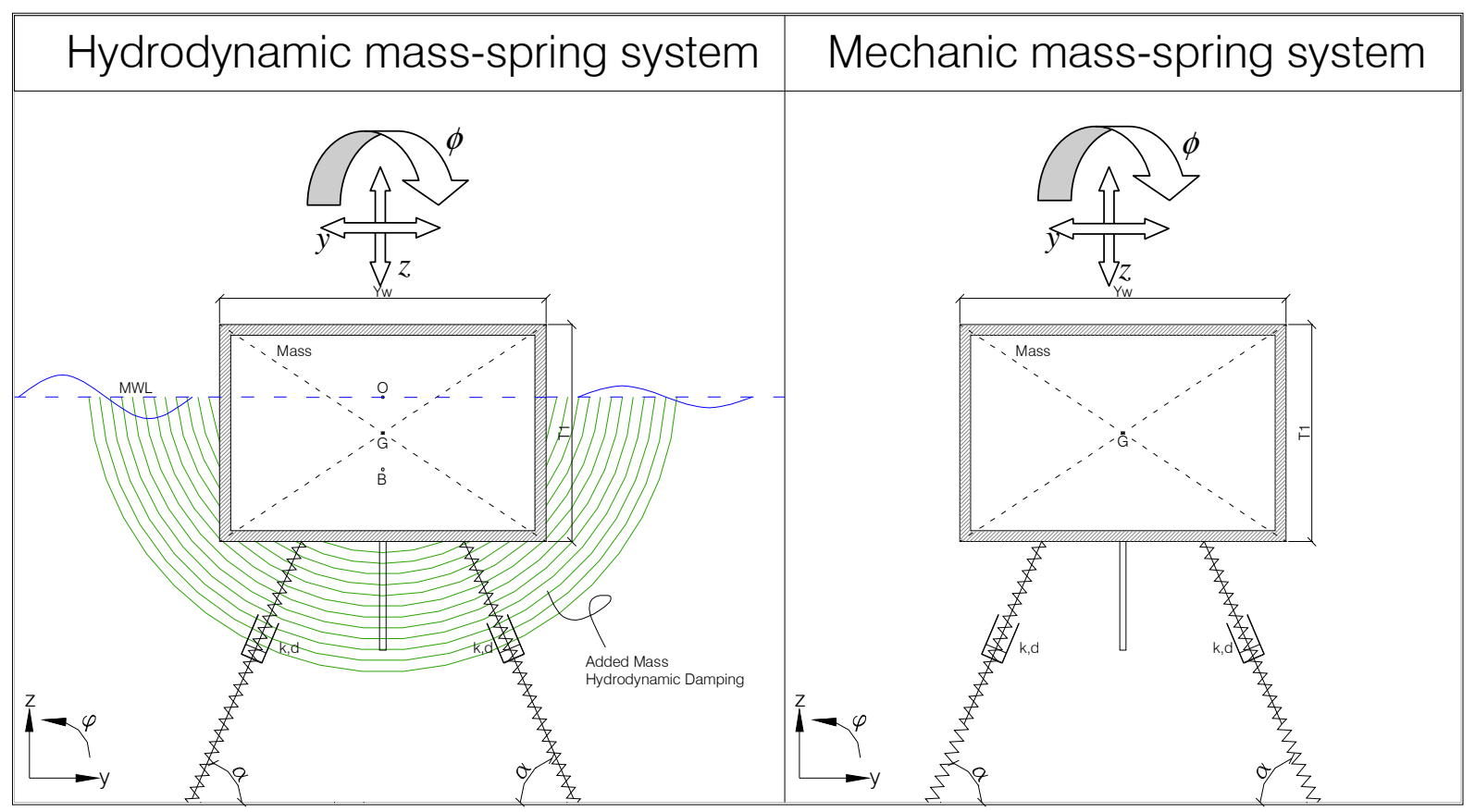

Figure 6-3: 2D representation of a hydrodynamic-c and a mechanic mass-spring system.

The heave natural frequency of a hydrodynamic system in a specific direction depends on the mass and the spring stiffness:

$\omega_{n}=\sqrt{\frac{\left(k+c_{n n}\right)}{\left(m+a_{n n}\right)}}$

Where:

$\begin{array}{llll}\omega_{n} & : & \text { Directional natural frequency } & (\mathrm{rad} / \mathrm{s}) \\ k_{m} & : & \text { Spring stiffness of the mooring line } & \left(\mathrm{kg} / \mathrm{s}^{2}\right) \\ m & : & \text { Structural mass } & (\mathrm{kg}) \\ c_{n n} & : & \text { Hydrodynamic spring stiffness } & \left(\mathrm{kg} / \mathrm{s}^{2}\right) \\ a_{n n} & : & \text { Hydrodynamic added mass } & (\mathrm{kg})\end{array}$

When the structure is under damped and the incoming wave frequency is close to the natural frequency of the system, resonance occurs. Figure 6-4 shows this phenomenon for a structure where the natural frequency is around $0.66[\mathrm{rad} / \mathrm{s}]$. Increasing the damping of the (hydro)dynamic system, results in a decrease of the resonance-peak. This phenomenon is visible in figure 6-4.

A shift of the resonance peak is achieved when the mass or the spring stiffness of the system is changed. This is due to the fact that a change of mass or spring stiffness results in a different natural frequency. When the mass of the system is increased, the resonance peak will shift to the low-frequency area of figure 6-4. Increasing the spring stiffness on the other hand will result in a shift of the resonance peak to the high-frequency area of figure 6-4. 


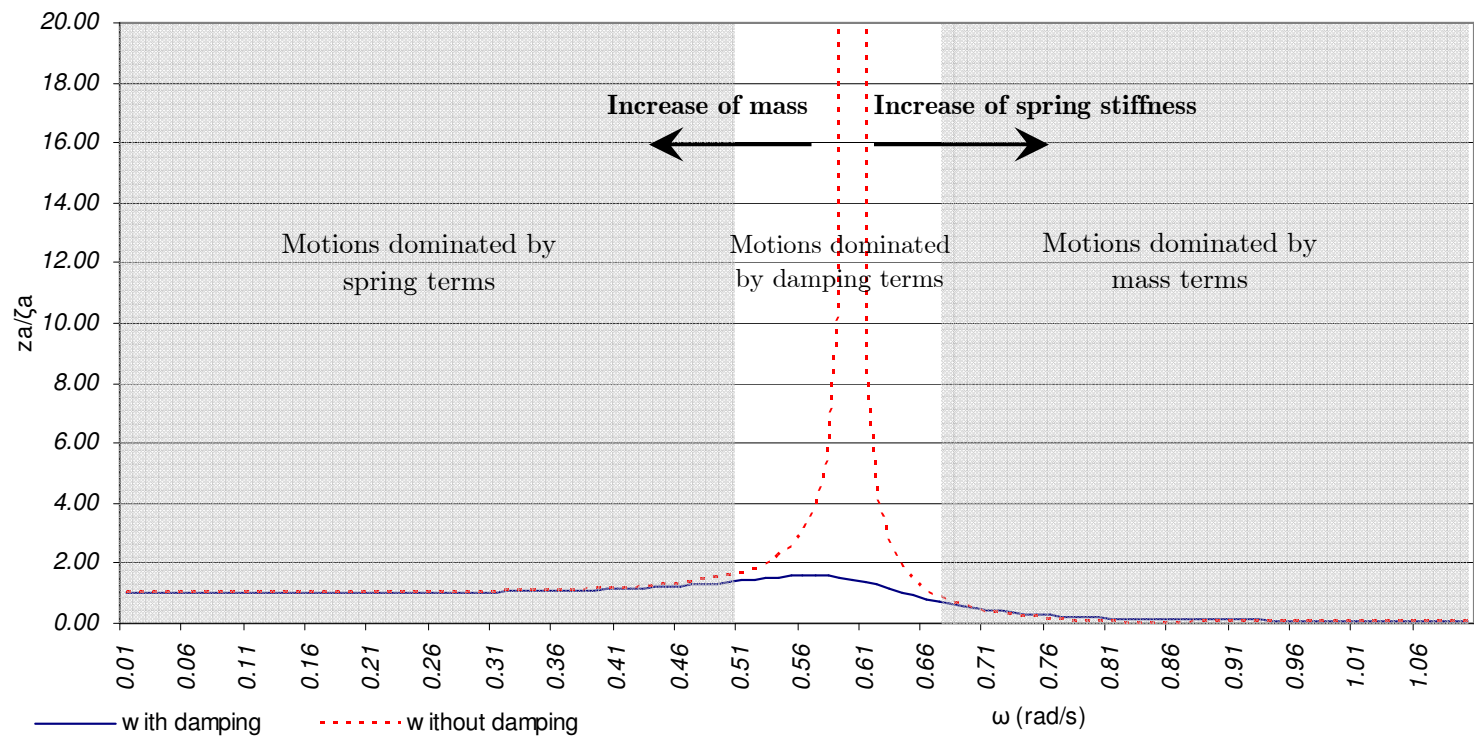

Figure 6-4: Influence of the damping, the mass and the spring stiffness on the dynamic behaviour of a mass-spring system. 


\subsection{Hydrodynamic calculation parameters}

In the previous paragraph the influence of the hydrodynamic parameters on the dynamic behaviour was already discussed. The magnitude of the hydrodynamic parameters does depend on the structural dimensions as well as the frequency of motion.

In this section, test results that were performed in the past are used to determine the magnitude of both hydrodynamic added mass and damping. The equations to calculate the hydrodynamic spring stiffness for the three two-dimensional motions are presented in section 6.2.3.

Appendix B.IV gives a detailed overview on how the hydrodynamic calculation parameters are obtained.

\subsubsection{Hydrodynamic mass coefficient}

The interaction of the oscillating (wetted) surface of the floating breakwater and the water particles accelerated by this oscillating surface, is called hydrodynamic mass or added mass. The hydromechanical reaction force, provided by this interaction, is direction-dependent. The hydromechanical reaction force does not dissipate energy and manifests itself as a standing wave near the surface of the floating breakwater.

As stated before, the hydrodynamic mass coefficient depends on several factors:

\begin{tabular}{|c|c|c|}
\hline & The dimensions of the floating breakwater: & $Y_{w} / L_{w} / T_{1} / T_{2}$ \\
\hline- & The wave frequency: & $\omega$ \\
\hline- & Water density: & $\rho$ \\
\hline
\end{tabular}

In his $\mathrm{PhD}$ thesis, J.H.Vugts ${ }^{1}$ did a lot of tests to state the relation between the factors as they are mentioned above and the hydrodynamic added mass. The added mass coefficients of heave $\left(\mathrm{a}_{\mathrm{zz}}\right)$ and sway $\left(\mathrm{a}_{\mathrm{yy}}\right)$ for several constructional lay-outs are reflected in the figures below.
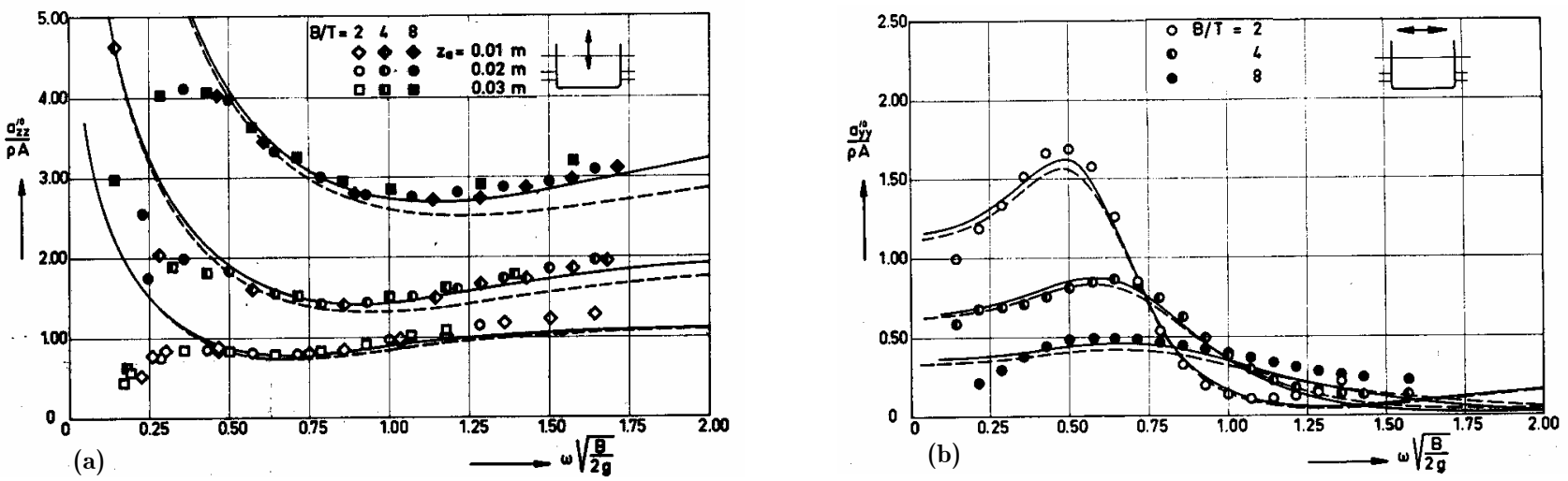

Figure 6-5: Added mass coefficients for heave (a) and sway (b). [J.H.Vugts $\left.{ }^{1}\right]$

Although Vugts performed tests to verify the magnitude of the roll added mass as well, they will not be used in this report. This is because of the fact that the tests were performed with fixed roll amplitudes. Since the roll amplitudes of the floating breakwater are not known on beforehand, the roll added mass moment of inertia is determined with the three-dimensional potential model DELFRAC.

The magnitudes of the sway added mass and the roll added mass moment of inertia will change when a screen is placed underneath the floating body. The effect of such a screen is estimated with the equation, prescribed by Newman ${ }^{2}$ for fully submerged bodies and checked with DELFRAC. This process is clarified in Appendix B.IV.

\footnotetext{
${ }^{1}$ Vugts, J.H. (1970). PhD thesis 'The hydrodynamic forces and ship motions in waves', Delft University of Technology.

${ }^{2}$ Newman J.N. (1977). 'Marine Hydrodynamics'. MIT Press, Cambridge, United Kingdom
} 


\subsubsection{Hydrodynamic damping coefficient}

The potential damping is related to the amount of energy the hydromechanical wave radiates from the oscillating floating breakwater at both sides of the structure. Since the waves withdraw energy from the oscillating floating breakwater, this is the reason why the motion will die out.

Since the magnitude of the hydrodynamic damping depends on the interaction of the oscillating (wetted) surface of the floating breakwater and the water particles, the same factors are involved as were mentioned in the previous section.

Besides the added mass, J.H.Vugts ${ }^{3}$ also performed tests to state the relation between the factors, as they are mentioned in the previous section, and the hydrodynamic damping. The hydrodynamic damping coefficients of heave $\left(b_{z z}\right)$ and sway $\left(b_{y y}\right)$ motions for several constructional lay-outs are reflected in figure 6-6.
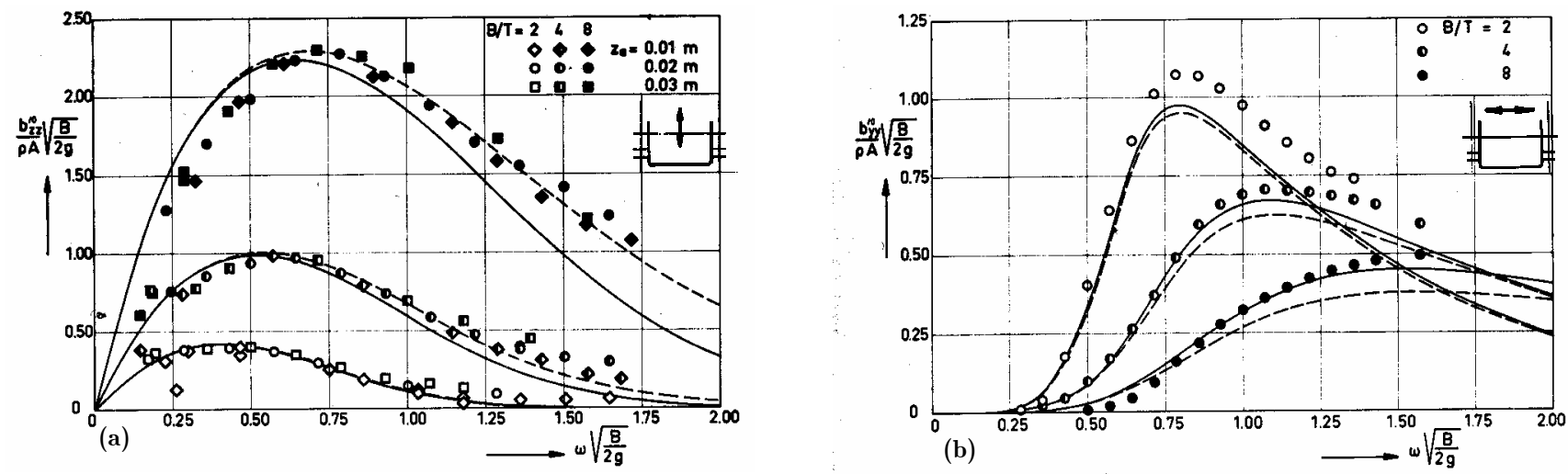

Figure 6-6: Damping coefficients for heave (a) and sway (b). [J.H.Vugts $\left.{ }^{3}\right]$

The roll damping coefficients are determined with DELFRAC for the same reasons as discussed in the previous section for the added mass moment of inertia.

A screen underneath the floating body of the breakwater will change the hydrodynamic damping coefficients of sway and roll. The influence of the screen is determined with DELFRAC. The process on how this is done is clarified in Appendix B.IV.

\footnotetext{
${ }^{3}$ Vugts, J.H. (1970). PhD thesis 'The hydrodynamic forces and ship motions in waves', Delft University of Technology.
} 
In order to use the Vugts data in a numerical model, the data are transferred into calculation polynomials. Since Vugts only gave data for rectangular cylinders with draft-width proportions of B/T=2, 4 and 8 , linear interpolation was performed to estimate the intermediate values. In this way, it is possible to perform more reliable calculations for a floating breakwater with a changing lay-out (width, draft, and mass). Graphs and approach formulas that have been used during the calculations are given in Appendix B.IV.

From the measurements as they were performed by Vugts, the added mass and damping coefficients are predictable in a defined width/draft and frequency range. This range, as it is stated for heave as well as sway is given in table $6-1$.

\begin{tabular}{l|ll}
\hline Motion & Dimension limit & Frequency limit \\
\hline Heave & $Y_{w} / T_{1} \leq 8$ & $0 \leq \omega \sqrt{\frac{Y_{w}}{2 g}} \leq 2$ \\
\hline Sway & $Y_{w} / T_{2} \geq 2$ & $0 \leq \omega \sqrt{\frac{Y_{w}}{2 g}} \leq 2$ \\
\hline Total & $2 \leq Y_{w} / T \leq 8$ & $0 \leq \omega \sqrt{\frac{Y_{w}}{2 g}} \leq 2$ \\
\hline
\end{tabular}

Table 6-1: Range limits.

\subsubsection{Hydrodynamic restoring spring coefficient}

The restoring force which pushes the floating breakwater back to its old position after an initial displacement is called the hydrodynamic spring coefficient. This force is produced by the shift in water pressure underneath the floating breakwater. According to this, hydrodynamic spring stiffness is only possible in motions with vertical components.

The hydrodynamic spring coefficient is a factor that depends on the following factors:

$\begin{array}{llll}- & : & \rho \\ \text { - } & \text { Submerged area of the floating breakwater section in the xy-plane } & : & A_{s} \\ \text { - } & \text { Submerged volume of the floating breakwater section } & & A_{w} \\ \text { - } & \text { Metacentric height } & : & \end{array}$

The hydrodynamic spring coefficients for heave, sway and roll become:

$$
\begin{aligned}
& c_{22}=0.00 \\
& c_{33}=\rho g A_{s} \\
& c_{44}=\rho g A_{w} \cdot \overline{G M}
\end{aligned}
$$




\subsection{Assumptions}

In the calculation model, a rectangular two-dimensional floating body will be considered. The floating body is able to move in the three degrees of freedom:

$\begin{array}{llll}\text { Horizontal } & : & \text { Sway } & (2) \\ \text { Vertical } & : & \text { Heave } & (3) \\ \text { Rotational } & : & \text { Roll } & (4)\end{array}$

Although these motions are all in the zy-plane, the calculation model can not be assumed to be two-dimensional. The dynamic behaviour of the system depends on the three-dimensional lay-out of the floating breakwater section.

Wave energy reduces exponentially in relation to the depth (eq.B-11 in Appendix B). This is why a wave with a high wave frequency will generate less vertical dynamic force underneath the floating breakwater compared to a low-frequency wave. The vertical wave energy in one wave period that the floating breakwater will encounter

is equal to:

$$
E_{Z}=e^{-k \cdot T_{1}} \cdot \frac{1}{2} \rho g \zeta_{a}^{2}
$$

The percentage of the total horizontal energy in a wave that the floating breakwater will encounter will be higher for a high-frequency wave. This higher percentage does not automatically result into a higher energy flux since the wave number (k) of high-frequency waves is larger. Figure 6-7 shows this effect.

The horizontal wave energy that the floating breakwater will encounter in one wave period is described as:

$$
E_{Y}=\left(1-e^{-k \cdot T_{2}}\right) \cdot \frac{1}{2} \rho g \zeta_{a}^{2}
$$

The part of the wave energy, present in the water underneath draft point $\mathrm{T}_{2}$, is assumed to radiate for $100 \%$ to the harbour side of the floating breakwater. The position of point $\mathrm{T}_{2}$ depends on the structural dimensions as well as the vertical motion amplitude $z_{a}$ of the floating breakwater. The amount of energy that transmits to the harbour side underneath point $\mathrm{T}_{2}$ is therefore described as:

$$
E_{T U}=\left(e^{-k \cdot\left(T_{2}-z_{a}\right)}\right) \cdot \frac{1}{2} \rho g \zeta_{a}^{2}
$$

The presence of a vertical screen underneath the structure is assumed not to have any influence on the vertical pressure built-up underneath the floating part of the floating breakwater. Although some influence might be expected, the gaps between the floating breakwater sections are assumed to be large enough to allow a $100 \%$ vertical pressure built-up. 


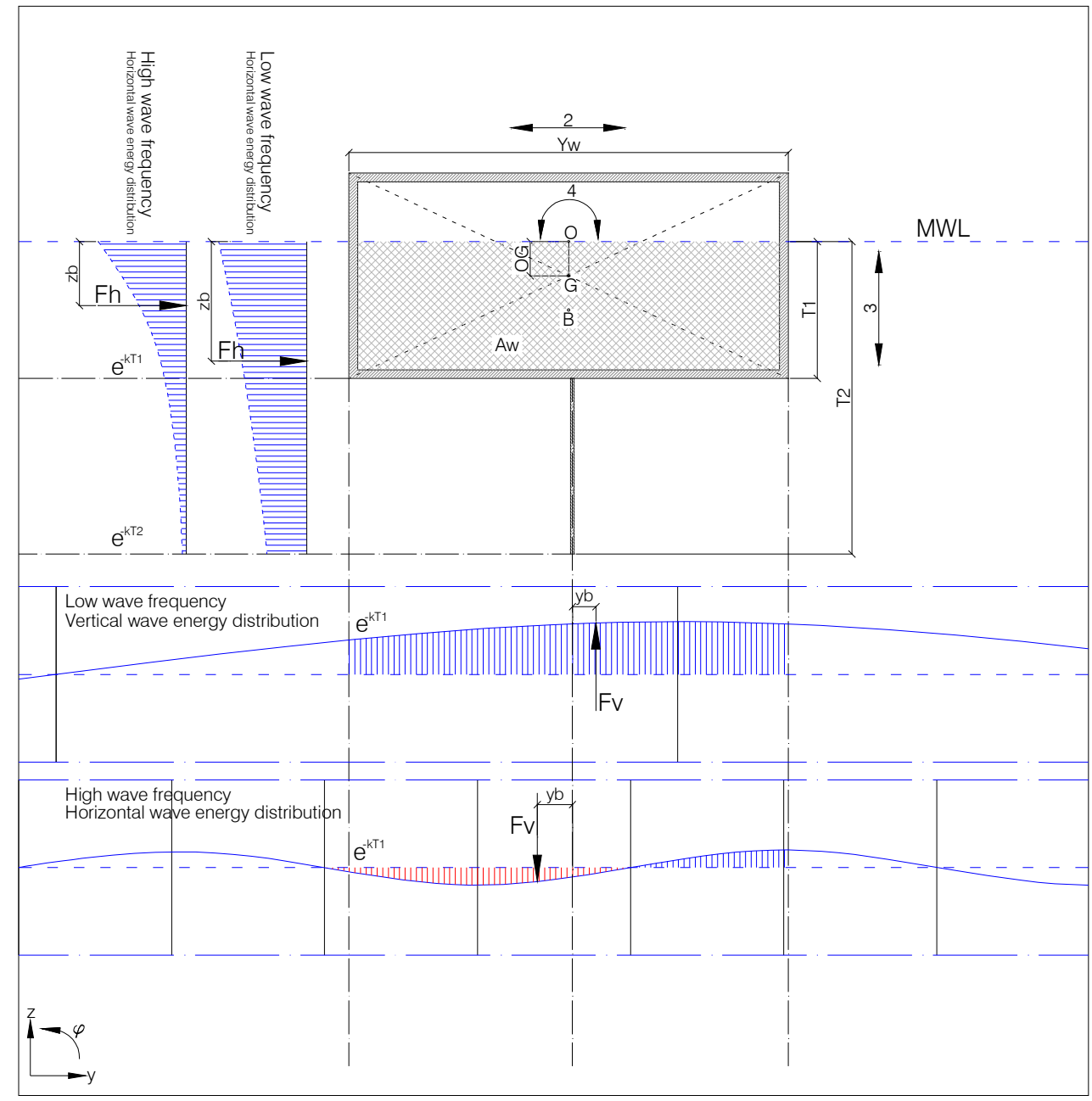

Figure 6-7: Frequency-related wave energy distribution at time step $t$.

The symbols used in figure 6-7 represent:

$A_{w} \quad: \quad$ Submerged volume of the floating breakwater section. $\quad\left[\mathrm{m}^{3}\right]$

$F_{v} \quad: \quad$ Resultant of the reduced vertical dynamic wave force

$[\mathrm{N}]$

$F_{h} \quad:$

Resultant of the reduced horizontal dynamic wave force

$[\mathrm{N}]$

Horizontal lever arm relative to the rotation point

$[\mathrm{m}]$

$\mathrm{y}_{\mathrm{b}} \quad:$

Vertical lever arm relative to the rotation point

$[\mathrm{m}]$

$\mathrm{O} \quad$ : $\quad$ Centre of gravity on the waterline

$[-]$

G : $\quad$ Centre of gravity of the body 


\subsection{Model setup}

The model calculations are performed in an Oyz coordinate system which is fixed in space. The y-axis in this coordinate system is parallel to the still water level. The z-axis is perpendicular positioned on the $y$-axis in vertical direction. The origin is the intersection of the $y$ and z-axis and is positioned on the centre of gravity of the floating breakwater.

During the calculations that will be performed, a clear distinction is made between the wave exciting forces and moments and the hydromechanical forces and moments. Wave exciting forces and moments are produced by the waves coming in on the floating breakwater. Hydromechanical forces and moments are produced by the motion of the floating breakwater itself.

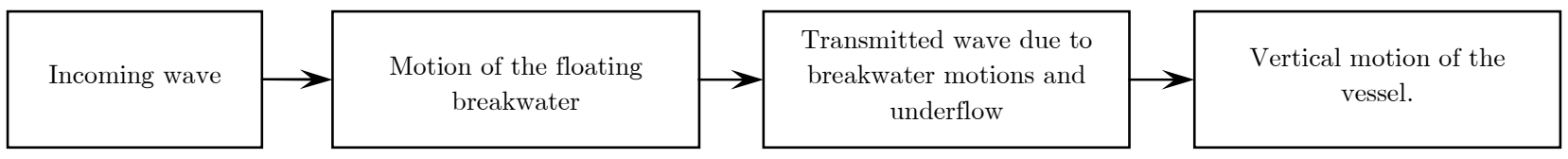

Figure 6-8: Wave force-structural motion relation

Figure 6-8 shows that there is a relation between these two forces and motions in the case of the floating breakwater. The incoming waves will put the structure into an oscillating motion. This motion itself will generate waves that will radiate from both sides of the structure.

In general, the motion of a two-dimensional linear system is described by a set of three coupled equations of motion. In a formal notation this set can be noted as:

\begin{tabular}{|c|c|c|}
\hline Sway & : & $\left(m+a_{22}\right) \ddot{y}+b_{22} \dot{y}+a_{24} \ddot{\phi}+b_{24} \dot{\phi}=F_{h}$ \\
\hline Heave & : & $\left(m+a_{33}\right) \ddot{z}+b_{33} \dot{z}+c_{33} z+a_{34} \ddot{\phi}+b_{34} \dot{\phi}+c_{34} \phi+a_{32} \ddot{y}+b_{32} \dot{y}=F_{v}$ \\
\hline Roll & & $\left(I_{x x}+a_{44}\right) \ddot{\phi}+b_{44} \dot{\phi}+c_{44} \phi+a_{42} \ddot{y}+b_{42} \dot{y}=K$ \\
\hline
\end{tabular}

Where

$m \quad: \quad$ Mass of the floating breakwater section

$I_{x x} \quad: \quad$ Mass moment of inertia about $\mathrm{G}$

$a_{i i} \quad: \quad$ Hydrodynamic mass in the i-mode of motion

$a_{i j} \quad: \quad$ Mass coupling coefficient in the i-equation of motion in the j-mode

$b_{i i} \quad: \quad$ Damping coefficient against motion in the i-mode of motion

$b_{i j} \quad: \quad$ Damping coupling coefficient in the i-equation of motion in the j-mode

$c_{i i} \quad: \quad$ Hydrostatic restoring coefficient against a displacement in the i-direction

$c_{i j} \quad: \quad$ Hydrostatic coupling coefficient in the i-equation by a displacement in the $\mathrm{j}$-direction

$K \quad$ : $\quad$ Moment around $\mathrm{O}$ when freely floating in waves or moment around $\mathrm{O}$ when forcedly oscillated in still water

In a matrix-notation, the coupled two-dimensional system can be described as:

$$
\left(\begin{array}{l}
F_{h} \\
F_{v} \\
K
\end{array}\right)=\left[\left(\begin{array}{ccc}
m & 0 & 0 \\
0 & m & 0 \\
0 & 0 & I
\end{array}\right)+\left(\begin{array}{lll}
a_{22} & a_{23} & a_{24} \\
a_{32} & a_{33} & a_{34} \\
a_{42} & a_{43} & a_{44}
\end{array}\right)\right] \cdot\left(\begin{array}{c}
\ddot{y} \\
\ddot{z} \\
\ddot{\phi}
\end{array}\right)+\left(\begin{array}{lll}
b_{22} & b_{23} & b_{24} \\
b_{32} & b_{33} & b_{34} \\
b_{42} & b_{43} & b_{44}
\end{array}\right) \cdot\left(\begin{array}{c}
\dot{y} \\
\dot{z} \\
\dot{\phi}
\end{array}\right)+\left(\begin{array}{lll}
c_{22} & c_{23} & c_{24} \\
c_{32} & c_{33} & c_{34} \\
c_{42} & c_{43} & c_{44}
\end{array}\right) \cdot\left(\begin{array}{c}
y \\
z \\
\phi
\end{array}\right)
$$


In equation 7.4 , the components $c_{22}, c_{32}, c_{42}$ are neglected since the horizontal displacement is not opposed by any restoring force. Lateral forces or moments can not be produced by the vertical motion of the floating breakwater. That is the reason why $a_{23}, b_{23}, c_{23}, a_{43}, b_{43}, c_{43}$ are left out of consideration as well.

In his report, $\mathrm{Vugts}^{4}$ proved that the $\phi$ - and y-components in the $\mathrm{F}_{\mathrm{v}}$-equation are small in respect to the z-components. Thus heaving becomes an uncoupled motion with one (vertical) degree of freedom.

In the following part of this research, heave, sway and roll are to be considered as uncoupled motions. The equations of motion for heave, sway and roll become:

Sway equation of motion:

$\left(\rho \nabla+a_{22}\right) \cdot \ddot{y}+\left(d_{m y}+b_{22}\right) \cdot \dot{y}+\left(k_{m y}\right) \cdot y=a_{22} \cdot \ddot{\zeta}_{w 2}^{*}+b_{22} \cdot \dot{\zeta}_{w 2}^{*}$

Heave equation of motion:

$\left(\rho \nabla+a_{33}\right) \cdot \ddot{z}+\left(d_{m z}+b_{33}\right) \cdot \dot{z}+\left(k_{m z}+c_{33}\right) \cdot z=a_{33} \cdot \ddot{\zeta}_{w 3}^{*}+b_{33} \cdot \dot{\zeta}_{w 3}^{*}+c_{33} \cdot \zeta_{w 3}^{*} \cdot C_{3}$

Roll equation of motion:

$\left(I_{x x}+a_{44}\right) \cdot \ddot{\phi}+\left(d_{m \phi}+b_{44}\right) \cdot \dot{\phi}+\left(k_{m \phi}+c_{44}\right) \cdot \phi=a_{44} \cdot \ddot{\zeta}_{w 4}^{*}+b_{44} \cdot \dot{\zeta}_{w 4}^{*}+c_{44} \cdot \zeta_{w 4}^{*}$

Where:

\section{Structural properties}

$\begin{array}{llll}y & : & \text { Displacement in the y-direction } & (\mathrm{m}) \\ \dot{y} & : & \text { Velocity in the y-direction } & (\mathrm{m} / \mathrm{s}) \\ \ddot{y} & : & \text { Acceleration in the y-direction } & \left(\mathrm{m} / \mathrm{s}^{2}\right) \\ z & : & \text { Displacement in the z-direction } & (\mathrm{m}) \\ \dot{z} & : & \text { Velocity in the z-direction } & (\mathrm{m} / \mathrm{s}) \\ \ddot{z} & : & \text { Acceleration in the z-direction } & \left(\mathrm{m} / \mathrm{s}^{2}\right) \\ \phi & : & \text { Rotation in the } \phi \text {-direction } & (\mathrm{rad}) \\ \dot{\phi} & : & \text { Velocity in the } \phi \text {-direction } & (\mathrm{rad} / \mathrm{s}) \\ \ddot{\phi} & : & \text { Acceleration in the } \phi \text {-direction } & \left(\mathrm{rad} / \mathrm{s}^{2}\right) \\ d_{m} & : & \text { Mooring damping in n-direction } & (\mathrm{Ns} / \mathrm{m}) \\ k_{m} & : & \text { Mooring spring stiffness in n-direction } & (\mathrm{N} / \mathrm{m}) \\ \nabla & : & \text { Volume of water displacement of the body in an equilibrium state } & \left(\mathrm{m}{ }^{3}\right) .\end{array}$

\section{Hydrodynamic properties}

$\begin{array}{llll}\zeta_{w n}^{*} & : & \text { Reduced wave elevation in the n-direction. } & (\mathrm{m}) \\ \dot{\zeta}_{w n}^{*} & : & \text { Reduced velocity of the water particles in the n-direction. } & (\mathrm{m} / \mathrm{s}) \\ \ddot{\zeta}_{w n}^{*} & : & \text { Reduced acceleration of the water particles in the n-direction. } & \left(\mathrm{m} / \mathrm{s}^{2}\right) \\ a_{n n} & : & \text { Hydrodynamic mass coefficient } & \left(\mathrm{Ns}{ }^{2} / \mathrm{m}\right) \\ b_{n n} & : & \text { Hydrodynamic damping coefficient } & (\mathrm{Ns} / \mathrm{m})\end{array}$

\footnotetext{
${ }^{4}$ Vugts, J.H. (1968). 'The hydrodynamic coefficients for swaying, heaving and rolling cylinders in a free surface', Netherlands ship research centre TNO.
} 


$\begin{array}{llll}c_{n n} & : & \text { Restoring spring coefficient } & (\mathrm{N} / \mathrm{m}) \\ \varepsilon_{n \zeta} & : & \text { Phase shift } & (\mathrm{deg} .) \\ C_{3} & : & \text { Vertical wave pressure distribution coefficient } & (-)\end{array}$

The wave parameters $\zeta_{w n}^{*}, \dot{\zeta}_{w n}^{*}$ and $\ddot{\zeta}_{w n}^{*}$ are called 'reduced' because of the development of the wave energy penetration into the depth (fig.6-7).

\subsubsection{Sway}

Sway is the horizontal motion that the structure will perform after it is loaded by the horizontal component of the incoming wave. Sway will generate a wave that is radiated to the harbour side as well as the sea side of the floating breakwater. Since the floating breakwater has a symmetric middle line, the simplified potential equation 6.4 can be applied. This means that the radiated wave at the ocean side of the floating breakwater has a phase difference of $\pi$ with the wave that is radiated to the leeward side.

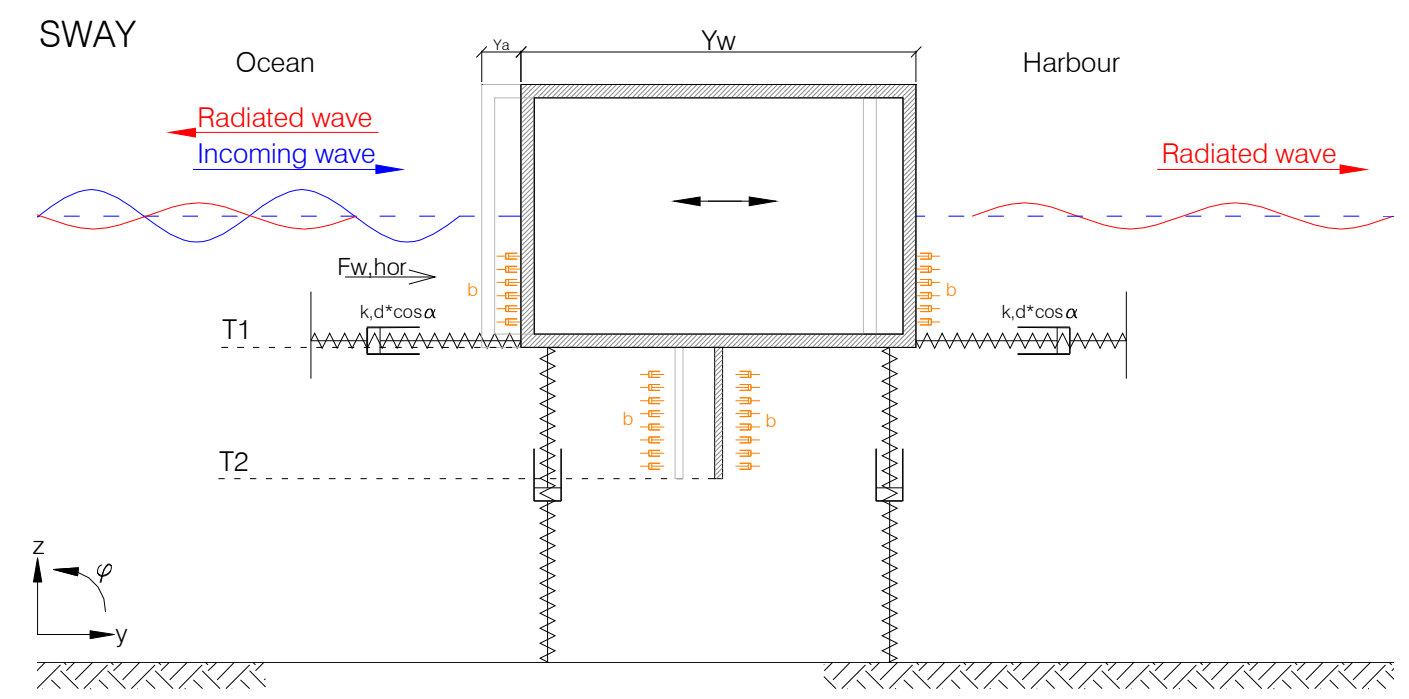

Figure 6-9: Hydrodynamic swaying system

The sway motion of the floating breakwater in the two-dimensional yz-plane is described with equation 7.5.

Equation 6.4 of the sway motion, based on the equation of motion deviates from the DELFRAC calculations as is shown in Appendix B. In very long waves the horizontal motion of a free-floating object, not influenced by wind, is expected to move with the same amplitude as the water particles do. In other words: when the floating object becomes very small compared to the wave length, the object will move with the same amplitude as the surrounding water particles.

However, in waves with smaller lengths, the floating breakwater is not able to maintain the same amplitude of motion as the water particles due to the mass of the structure (Newton's second law).

The horizontal wave force can be written as the sum of a so-called inertia force and a drag force.

$F_{w ; h}=\left\{a_{22} \cdot \ddot{\zeta}_{w 2}^{*}+b_{22} \cdot \dot{\zeta}_{w 2}^{*}\right\}+\left\{\rho \cdot A_{z} \cdot C_{w} \cdot\left|\dot{\zeta}_{w 2}^{*}-\dot{y}\right| \cdot\left(\dot{\zeta}_{w 2}^{*}-\dot{y}\right)\right\}^{5}$

\footnotetext{
${ }^{5}$ Grotmaack, R and Meylan, M.H. "Wave forcing of small floating bodies" Institute of Information and Mathematical Sciences, Massey Univ. Auckland, New Zealand.
} 
Where:
$A_{z} \quad:$
Surface area of the moving body
$C_{w} \quad:$
Drag coefficient

As can be concluded from equation 7.5, the drag force is not included in the calculation model. The motion, generated by the drag force is not included in this research due to the influence of the mooring system. As was stated in chapter 5.2.2, the mooring system does not influence the hydrodynamic behaviour on the short term, but it will hold the floating breakwater system on its position when long waves are considered.

The motion-depending calculation parameters in equation 6.11 are:

\begin{tabular}{|c|c|c|c|c|c|}
\hline$a_{22}$ & : & Sway added mass & $\left(\mathrm{Ns}^{2} / \mathrm{m}=\mathrm{kg}\right)$ & & \\
\hline$b_{22}$ & : & Sway hydrodynamic damping & $(\mathrm{Ns} / \mathrm{m}=\mathrm{kg} / \mathrm{s})$ & & \\
\hline$d_{m y}$ & : & Mooring damping in the horizontal direction. & $(\mathrm{Ns} / \mathrm{m}=\mathrm{kg} / \mathrm{s})$ & $=d_{m} \cdot \cos \alpha$ & \\
\hline$k_{m y}$ & $:$ & Mooring spring stiffness in the horizontal dir. & $\left(\mathrm{N} / \mathrm{m}=\mathrm{kg} / \mathrm{s}^{2}\right)$ & $=k_{m} \cdot \cos \alpha$ & \\
\hline$y$ & : & Displacement in the $\mathrm{y}$-direction & $(\mathrm{m})$ & $=-y_{a} \cos \left(\omega t+\varepsilon_{y \zeta}\right)$ & \\
\hline$\dot{y}$ & : & Velocity in the y-direction & $(\mathrm{m} / \mathrm{s})$ & $=y_{a} \omega \sin \left(\omega t+\varepsilon_{y \zeta}\right)$ & $(6.15)$ \\
\hline$\ddot{y}$ & : & Acceleration in the y-direction & $\left(\mathrm{m} / \mathrm{s}^{2}\right)$ & $=y_{a} \omega^{2} \cos \left(\omega t+\varepsilon_{y \zeta}\right)$ & \\
\hline$\zeta_{w 2}^{*}$ & : & Depth-related reduced wave elevation. & & $=-e^{-k T_{2}} \cdot \zeta_{a} \cos (\omega t)$ & \\
\hline$\dot{\zeta}_{w 2}^{*}$ & : & Depth-related velocity of the water particles. & & $=e^{-k T_{2}} \cdot \zeta_{a} \cdot \omega \sin (\omega t)$ & $(6.16)$ \\
\hline$\ddot{\zeta}_{w 2}^{*}$ & $:$ & Depth-related acceleration of the water particles. & & $=e^{-k T_{2}} \cdot \zeta_{a} \cdot \omega^{2} \cos (\omega t)$ & \\
\hline$\alpha$ & : & Mooring line angle & $\left({ }^{\circ}\right)$ & & \\
\hline
\end{tabular}

Equations 6.15 and 6.16 are clarified in Appendix B, chapter B.V. In chapter B.V.c of Appendix B, the equations of the horizontal wave force amplitude and the sway motion amplitude are derived.

The horizontal wave force amplitude is noted as:

$$
F_{a ; y}=\zeta_{a} \cdot\left(1-e^{-k T_{2}}\right) \sqrt{\left\{a_{22} \omega^{2}\right\}^{2}+\left\{b_{22} \omega\right\}^{2}}
$$

Due to this wave force, the floating breakwater is put into an oscillating motion with amplitude:

$$
y_{a}=\zeta_{a} \cdot\left(e^{-k T_{2}}\right) \sqrt{\frac{\left\{a_{22} \omega^{2}\right\}^{2}+\left\{b_{22} \omega\right\}^{2}}{\left\{-k+\left(m+a_{22}\right) \omega^{2}\right\}^{2}+\left\{\left(d+b_{22}\right) \omega\right\}^{2}}}
$$

An undisturbed swaying hydrodynamic system is over damped since the horizontal hydrodynamic spring stiffness is assumed to be zero. However, when the horizontal spring stiffness of the mooring system increases, it is possible that an under damped situation occurs. The natural frequency of a swaying hydrodynamic system becomes:

$$
\omega_{n ; y}=\sqrt{\frac{\left(k_{m y}\right)}{\left(m+a_{22}\right)}}
$$


A combination of equations 6.15 and 6.18 yields the total sway oscillation of the system in time:

$$
y(t)=-\zeta_{a} \cdot\left(e^{-k T_{2}}\right) \sqrt{\frac{\left\{a_{22} \omega^{2}\right\}^{2}+\left\{b_{22} \omega\right\}^{2}}{\left\{-k+\left(m+a_{22}\right) \omega^{2}\right\}^{2}+\left\{\left(d+b_{22}\right) \omega\right\}^{2}}} \cdot \cos \left(\omega t+\varepsilon_{y \zeta}\right)
$$

Where the phase shift of the sway motion is:

$$
\varepsilon_{y \zeta}=\arctan \left\{\frac{m b_{22} \omega^{3}}{\left(a_{22} \omega^{2}\right)\left\{-k+\left(m+a_{22}\right) \omega^{2}+\left\{\left(d+b_{22}\right) \omega\right\}^{2}\right\}}\right\} \quad \text { with: } 0 \leq \varepsilon_{y \zeta} \leq 2 \pi
$$

The sway motion of the floating breakwater generates waves. These generated waves subtract kinetic energy from the oscillating floating breakwater. In Appendix B it is proven that the hydrodynamic damping is the reason why the oscillating motion dies out. The energy balance of the horizontal damping component and the radiated wave results into the equation that determines the amplitude of the sway radiated wave.

$$
\zeta_{T ; \text { sway }}(t)=\sqrt{\frac{b_{22} \cdot \omega^{3} \cdot y_{a}^{2}}{\rho \cdot g^{2}}} \cdot \sin \left(\omega t+\varepsilon_{y \zeta}\right)
$$




\subsubsection{Heave}

Heave is the vertical motion that the structure will perform after it is loaded by the incoming wave. The wave generated by the heave motion of the floating breakwater radiates to the harbour side with a phase difference compared to the incoming wave. This phase difference depends on the structural dimensions, the wave frequency and the mooring stiffness. According to the simplified potential equation 6.4 , the radiated waves at both sides of the floating breakwater have no mutual phase difference.

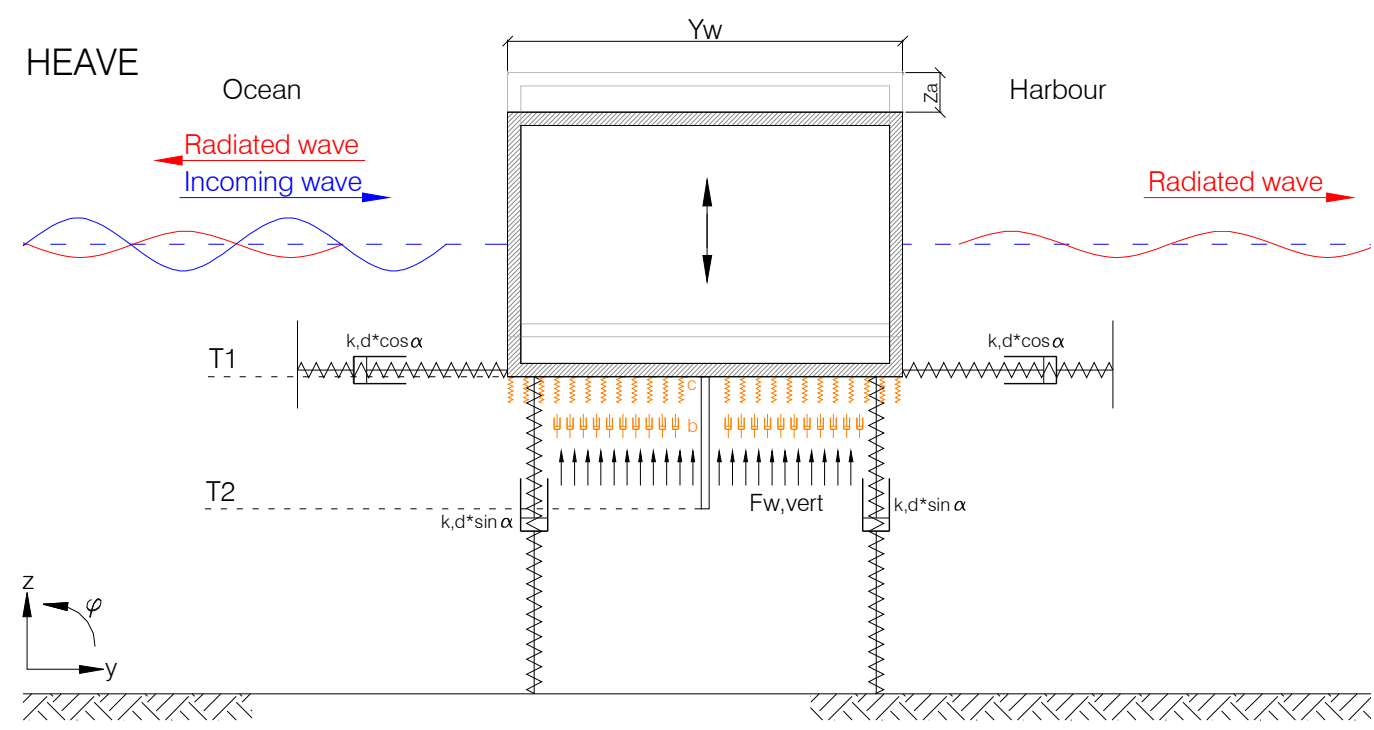

Figure 6-10: Hydrodynamic heaving system

The heave motion of the floating breakwater in the two-dimensional yz-plane is described by equation 6.12 . The motion-depending calculation parameters in this formula are:

\begin{tabular}{|c|c|c|c|c|c|}
\hline$a_{33}$ & $:$ & Heave added mass & $\left(\mathrm{Ns}^{2} / \mathrm{m}=\mathrm{kg}\right)$ & & \\
\hline$b_{33}$ & : & Hydrodynamic damping & $(\mathrm{Ns} / \mathrm{m}=\mathrm{kg} / \mathrm{s})$ & & \\
\hline$c_{33}$ & $:$ & Vertical restoring spring coefficient & $\left(\mathrm{N} / \mathrm{m}=\mathrm{kg} / \mathrm{s}^{2}\right)$ & $=\rho g A_{s}$ & \\
\hline$C_{3}$ & $:$ & Vertical wave pressure distribution coefficient & & $=1-\frac{k}{Y_{w}} \cdot e^{-k T_{1}}$ & \\
\hline$d_{m z}$ & : & Mooring damping in the vertical direction. & $(\mathrm{Ns} / \mathrm{m}=\mathrm{kg} / \mathrm{s})$ & $=d \cdot \sin \alpha$ & \\
\hline$k_{m z}$ & $:$ & Mooring spring stiffness in the vertical direction. & $\left(\mathrm{N} / \mathrm{m}=\mathrm{kg} / \mathrm{s}^{2}\right)$ & $=k \cdot \sin \alpha$ & \\
\hline$z$ & $:$ & Displacement in the z-direction & $(\mathrm{m})$ & $=z_{a} \sin \left(\omega t+\varepsilon_{z \zeta}\right)$ & \\
\hline$\dot{z}$ & : & Velocity in the z-direction & $(\mathrm{m} / \mathrm{s})$ & $=z_{a} \omega \cos \left(\omega t+\varepsilon_{z \zeta}\right)$ & $(6.23)$ \\
\hline$\ddot{z}$ & : & Acceleration in the z-direction & $\left(\mathrm{m} / \mathrm{s}^{2}\right)$ & $=-z_{a} \omega^{2} \sin \left(\omega t+\varepsilon_{z \zeta}\right)$ & \\
\hline$\zeta_{w 3}^{*}$ & : & Reduced wave elevation. & $(\mathrm{m})$ & $=e^{-k T_{1}} \cdot \zeta_{a} \sin (\omega t)$ & \\
\hline$\dot{\zeta}_{w 3}^{*}$ & : & Velocity of the water particles. & $(\mathrm{m} / \mathrm{s})$ & $=e^{-k T_{1}} \cdot \zeta_{a} \cdot \omega \cos (\omega t)$ & (6.24) \\
\hline$\ddot{\zeta}_{w 3}^{*}$ & : & Acceleration of the water particles. & $\left(\mathrm{m} / \mathrm{s}^{2}\right)$ & $=-e^{-k T_{1}} \cdot \zeta_{a} \cdot \omega^{2} \sin (\omega t)$ & \\
\hline
\end{tabular}

Equations 6.23 and 6.24 are clarified in Appendix B, chapter B.V. In chapter B.V.d of Appendix B, the equations of the vertical wave force amplitude and the heave motion amplitude are derived. 
The vertical wave force amplitude is noted as:

$$
F_{a}=\zeta_{a} \cdot e^{-k T_{1}} \sqrt{\left\{c_{33} \cdot C_{3}-a_{33} \omega^{2}\right\}^{2}+\left\{b_{33} \omega\right\}^{2}}
$$

Due to this wave force, the floating breakwater is put into a vertical oscillating motion with amplitude:

$$
z_{a}=\zeta_{a} \cdot e^{-k T_{1}} \sqrt{\frac{\left\{c_{33} \cdot C_{3}-a_{33} \omega^{2}\right\}^{2}+\left\{b_{33} \omega\right\}^{2}}{\left\{\left(k_{m z}+c_{33}\right)-\left(m+a_{33}\right) \omega^{2}\right\}^{2}+\left\{\left(d_{m z}+b_{33}\right) \omega\right\}^{2}}}
$$

As explained in chapter 6.1.2, the parameters that determine the natural frequency can be used to optimize the floating breakwater performance. The natural frequency of a heaving hydrodynamic system is given as:

$$
\omega_{n ; z}=\sqrt{\frac{\left(k_{m z}+c_{33}\right)}{\left(m+a_{33}\right)}}
$$

A combination of equations 6.23 and 6.26 yields the total heave oscillation of the system in time:

$$
z(t)=\zeta_{a} \cdot e^{-k T_{1}} \sqrt{\frac{\left\{c_{33} \cdot C_{3}-a_{33} \omega^{2}\right\}^{2}+\left\{b_{33} \omega\right\}^{2}}{\left\{\left(k_{m z}+c_{33}\right)-\left(m+a_{33}\right) \omega^{2}\right\}^{2}+\left\{\left(d_{m z}+b_{33}\right) \omega\right\}^{2}}} \cdot \sin \left(\omega t+\varepsilon_{z \zeta}\right)
$$

Where the phase shift of the heave motion is:

$$
\varepsilon_{z \zeta}=\arctan \left\{\frac{-m b_{33} \omega^{3}}{\left(c_{33} \cdot C_{3}-a_{33} \omega^{2}\right)\left\{\left(k_{m z}+c_{33}\right)-\left(m+a_{33}\right) \omega^{2}+\left\{\left(d_{m z}+b_{33}\right) \omega\right\}^{2}\right\}}\right\} \quad \text { with: } 0 \leq \varepsilon_{y \zeta} \leq 2 \pi
$$

The heave motion of the floating breakwater generates waves. The same theory as was explained for the sway motion system holds for the determination of the radiated wave amplitude generated by the heave motion. The transmitted wave amplitude generated by the vertical motion becomes:

$$
\zeta_{T ; \text { heave }}(t)=\sqrt{\frac{b_{33} \cdot \omega^{3} \cdot z_{a}^{2}}{\rho \cdot g^{2}}} \cos \left(\omega t+\varepsilon_{z \zeta}\right)
$$




\subsubsection{Roll}

Roll occurs when the resultant of the hydrodynamic forces is not in line with the centre of rotation. In other words: roll is the hydrodynamic reaction on the wave exciting moments. The horizontal and vertical wave exciting forces times the relevant lever arms produce these moments. The roll motion itself creates a standing wave on the ocean side of the floating breakwater with a phase difference compared to the incoming wave. This phase difference depends on the structural dimensions, the wave frequency and the mooring stiffness. The radiated wave at the leeward side is in most cases in phase with the incoming wave.

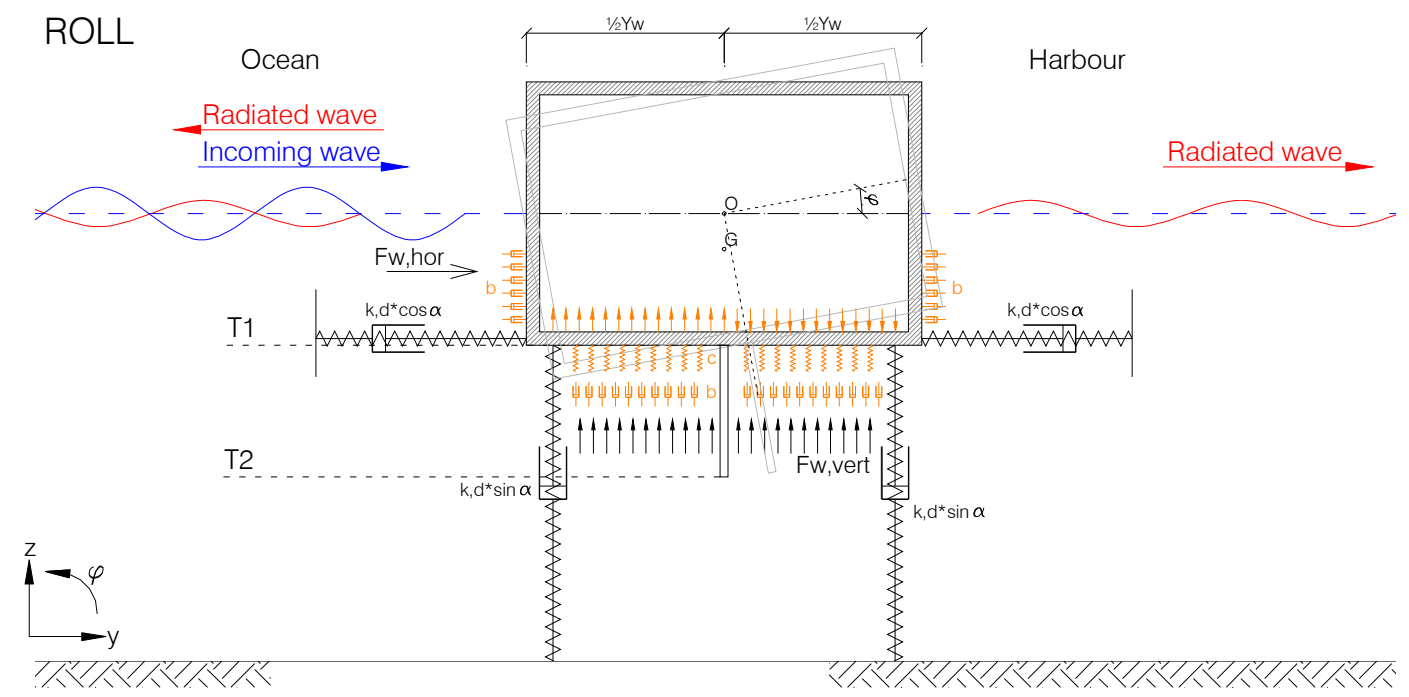

Figure 6-11: Hydrodynamic rolling system

Roll of the floating breakwater in the two-dimensional yz-plane is described with equation 7.13. With the properties of figure 6 - 11 , this equation has to be evolved to:

$\left(I+a_{44}\right) \cdot \ddot{\phi}+\left(1 / 2 Y_{w}^{2} \cdot d_{b z}+2 T_{1}^{2} \cdot d_{b y}+b_{44}\right) \cdot \dot{\phi}+\left(1 / 2 Y_{w}^{2} \cdot k_{c z}+2 T_{1}^{2} \cdot k_{c y}+c_{44}+\overline{O G} \cdot m g\right) \cdot \phi=F_{w ; h o r} \cdot z_{b}+F_{w ; v e r t} \cdot y_{b}$

The motion-depending calculation parameters in this formula are:

\begin{tabular}{|c|c|c|c|c|}
\hline$a_{44}$ & : & Added mass moment of inertia & $\left(\mathrm{Ns}^{2} / \mathrm{rad}\right)$ & \\
\hline$b_{44}$ & : & Hydraulic roll damping & $(\mathrm{Ns} / \mathrm{rad})$ & \\
\hline$c_{44}$ & : & Water spring stiffness & $(\mathrm{N} / \mathrm{rad})$ & $=\rho g Y_{w} \cdot \overline{G M}$ \\
\hline$\overline{G M}$ & $:$ & Transverse metacentric height & $(\mathrm{m})$ & $=\overline{B M}-\overline{B G}$ \\
\hline & & & & $\frac{1}{12} \cdot Y_{w}^{3}$ \\
\hline$B M$ & : & Distance between centre of buoyancy and metacentre & $(\mathrm{m})$ & $=\frac{12}{Y_{w} \cdot T_{1}}$ \\
\hline$B G$ & $:$ & Distance between the centres of gravity and buoyancy & $(\mathrm{m})$ & \\
\hline$O G$ & : & Distance of centre of gravity below the waterline & $(\mathrm{m})$ & \\
\hline$I_{x x}$ & : & Mass moment of inertia about $\mathrm{G}$ per $\mathrm{m}^{1}$ & & $=k_{x x}^{2} \cdot m=\left(0.35 \cdot Y_{w}\right)^{2} \cdot m$ \\
\hline$\phi$ & $:$ & Rotation about the $\mathrm{x}$-axis & $(\mathrm{rad})$ & $=-\phi_{a} \cos \left(\omega t+\varepsilon_{\phi \zeta}\right)$ \\
\hline$\dot{\phi}$ & : & Velocity of rotation about the $\mathrm{x}$-axis & $(\mathrm{rad} / \mathrm{s})$ & $=\phi_{a} \omega \cdot \sin \left(\omega t+\varepsilon_{\phi \zeta}\right)$ \\
\hline$\ddot{\phi}$ & $:$ & Acceleration of rotation about the $\mathrm{x}$-axis & $\left(\mathrm{rad} / \mathrm{s}^{2}\right)$ & $=\phi_{a} \omega^{2} \cdot \cos \left(\omega t+\varepsilon_{\phi \zeta}\right)$ \\
\hline$y_{b}$ & : & Horizontal lever arm, relative to the rotation origin. & $(\mathrm{m})$ & \\
\hline$z_{b}$ & : & Vertical lever arm, relative to the rotation origin. & $(\mathrm{m})$ & \\
\hline
\end{tabular}


Equation 6.31 is clarified in Appendix B, chapter B.V. In chapter B.V.f of Appendix B, the equations of the wave exciting moment amplitude and the roll motion amplitude are derived.

The amplitude of the wave exciting moment is denoted as:

$$
M_{a}=\zeta_{a} \cdot \sqrt{\left[e^{-k T_{2}} \cdot z_{b} \cdot\left\{a_{22} \omega^{2}\right\}+e^{-k T_{1}} \cdot y_{b} \cdot\left\{b_{33} \omega\right\}\right]^{2}+\left[e^{-k T_{2}} \cdot z_{b} \cdot\left\{b_{22} \omega\right\}-e^{-k T_{1}} \cdot y_{b} \cdot\left\{c_{33} \cdot C_{3}-a_{33} \omega^{2}\right\}\right]^{2}}
$$

Due to this moment, the floating breakwater is put into an oscillating roll motion with amplitude:

$$
\phi_{a}=\zeta_{a} \cdot \sqrt{\frac{\left\{\left(e^{-k T_{2}} \cdot z_{b} \cdot a_{22} \omega^{2}\right)+\left(e^{-k T_{1}} \cdot y_{b} \cdot b_{33} \omega\right)\right\}^{2}+\left\{\left(e^{-k T_{2}} \cdot z_{b} \cdot b_{22} \omega\right)-\left(y_{b} \cdot e^{-k T_{1}}\left(c_{33} \cdot C_{3}-a_{33} \omega^{2}\right)\right)\right\}^{2}}{\left\{\left(I+a_{44}\right) \omega^{2}-\left(1 / 2 Y_{w}^{2} \cdot k_{c z}+2 T_{1}^{2} \cdot k_{c y}+c_{44}+\overline{O G} \cdot m g\right)\right\}^{2}+\left\{\left(1 / 2 Y_{w}^{2} \cdot d_{b z}+2 T_{1}^{2} \cdot d_{b y}+b_{44}\right) \omega\right\}^{2}}}
$$

A floating body which is not influenced by external factors, is an under damped hydrodynamic system when the rotational motions are considered. The natural frequency of such a rotational, or rolling, system is described by:

$$
\omega_{n 4}=\sqrt{\left(\frac{\rho g \cdot A_{w} \cdot \overline{G M}_{T}}{m \cdot k_{44}^{2}+a_{44}}\right)}
$$

Where:

$k_{44} \approx 0.35 \cdot Y_{w}$

A combination of equations 6.31 and 6.33 yields the total roll oscillation of the system in time:

$$
\phi(t)=\zeta_{a} \cdot \sqrt{\frac{\left\{\left(e^{-k T_{2}} \cdot z_{b} \cdot a_{22} \omega^{2}\right)+\left(e^{-k T_{1}} \cdot y_{b} \cdot b_{33} \omega\right)\right\}^{2}+\left\{\left(e^{-k T_{2}} \cdot z_{b} \cdot b_{22} \omega\right)-\left(y_{b} \cdot e^{-k T_{1}}\left(c_{33} \cdot C_{3}-a_{33} \omega^{2}\right)\right)\right\}^{2}}{\left\{\left(I_{x x}+a_{44}\right) \omega^{2}-\left(1 / 2 Y_{w}^{2} \cdot k_{c z}+2 T_{1}^{2} \cdot k_{c y}+c_{44}+\overline{O G} \cdot m g\right)\right\}^{2}+\left\{\left(1 / 2 Y_{w}^{2} \cdot d_{b z}+2 T_{1}^{2} \cdot d_{b y}+b_{44}\right) \omega\right\}^{2}} \cdot \cos \left(\omega t+\varepsilon_{\phi \zeta}\right)}
$$

The derivative process to obtain the phase shift of the roll motion is clarified in Appendix B. The comprehensive equation of the roll phase shift is given in equation B-85 of this appendix.

The roll motion of the floating breakwater generates waves. The same theory as was explained for the sway motion system holds for the determination of the radiated wave amplitude generated by the roll motion. The transmitted wave amplitude generated by the rotational motion becomes:

$$
\zeta_{T ; \text { roll }}(t)=-\sqrt{\frac{b_{44} \cdot \omega^{3} \cdot \phi_{a}^{2}}{L_{w} \cdot \rho \cdot g^{2}}} \cdot \sin \left(\omega t+\varepsilon_{\phi \zeta}\right)
$$




\subsubsection{Underflow}

The part of the wave energy that is not influenced by the presence of the floating breakwater is from now on called the underflow. This phenomenon was already discussed in chapter 6.3 , where it was stated that $100 \%$ of the wave energy present underneath draft point $\mathrm{T}_{2}$ is assumed to radiate for $100 \%$ to the leeward side of the floating breakwater.

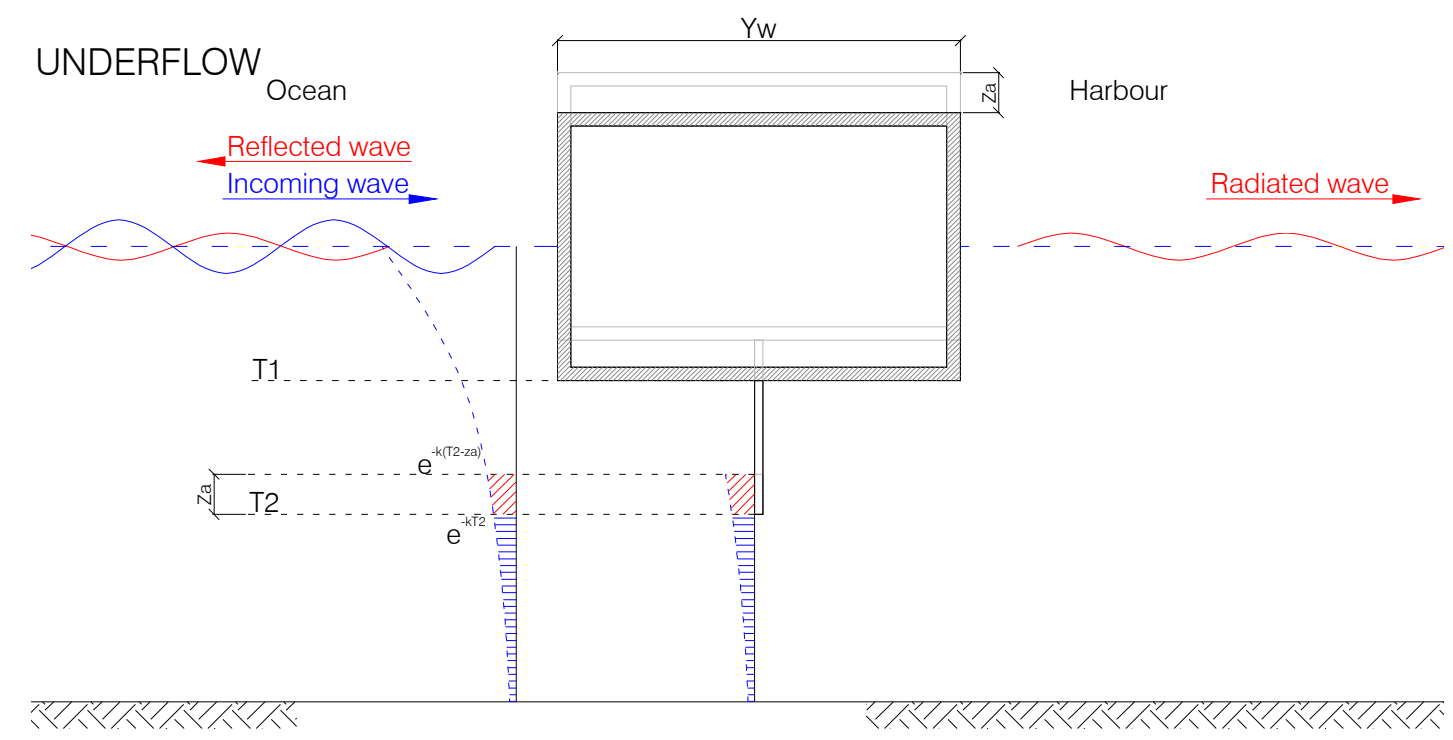

Figure 6-12: The underflow phenomenon.

With this assumption, the radiated wave amplitude can be calculated with the energy balance.

$E_{\text {underflow }}=E_{\text {Transmitted }}$

With this energy balance, the transmitted wave amplitude due to the underflow becomes:

$\zeta_{T ; U}=\sqrt{e^{-k\left(T_{2}-z_{a}\right)} \cdot \zeta_{a}^{2}}$ 


\subsubsection{Total wave transmission}

The total transmitted wave amplitude consists of a summation of all the amplitudes that are radiated by the motions and the underflow. This total transmitted wave amplitude has to be small enough to guarantee $100 \%$ harbour efficiency. In table 5-1 the maximum allowed motions in a harbour were defined. The relative heave amplitude between the FTCT and the moored vessel should be smaller than $0.40 \mathrm{~m}$.

In Appendix B, chapter B.VI the equation of the total transmitted wave amplitude is defined as:

$$
\zeta_{T}=\left|\zeta_{T ; \text { heave }}+\zeta_{T ; U}+\zeta_{T ; \text { sway }}+\zeta_{T ; \text { roll }}\right| \leq \zeta_{T ; s}
$$

Where:

$\zeta_{T ; s} \quad: \quad$ The maximum allowed wave amplitude at the leeward side of the floating breakwater.

The dynamic reaction of the vessel on this maximum allowed wave amplitude depends on the ship dimensions, as shown in figure 5-2. The allowed wave amplitude can be calculated with:

$$
\zeta_{T ; s}=\frac{z_{s}}{e^{-k T_{s}} \cdot \sqrt{\frac{\left\{c_{s}-a_{s} \omega^{2}\right\}^{2}+\left\{b_{s} \omega\right\}^{2}}{\left\{c_{s}-\left(m_{s}+a_{s}\right) \omega^{2}\right\}^{2}+\left\{b_{s} \omega\right\}^{2}}}}
$$

Where:

$\begin{array}{llll}a_{s} & : & \text { Heave added mass of the vessel } & \left(\mathrm{Ns}^{2} / \mathrm{m}\right) \\ b_{s} & : & \text { Hydraulic damping of the vessel } & (\mathrm{Ns} / \mathrm{m}) \\ c_{s} & : & \text { Vertical restoring spring stiffness } & (\mathrm{N} / \mathrm{m})=\rho g \cdot A_{s ; \text { vessel }} \\ z_{s} & : & \text { Maximum allowed heave amplitude } & (\mathrm{m})=0.40 \\ T_{s} & : & \text { Draft of the vessel } & (\mathrm{m})\end{array}$

A combination of equations $6.22,6.30,6.37,6.38$ and 6.40 yields the equation of the total transmitted wave amplitude.

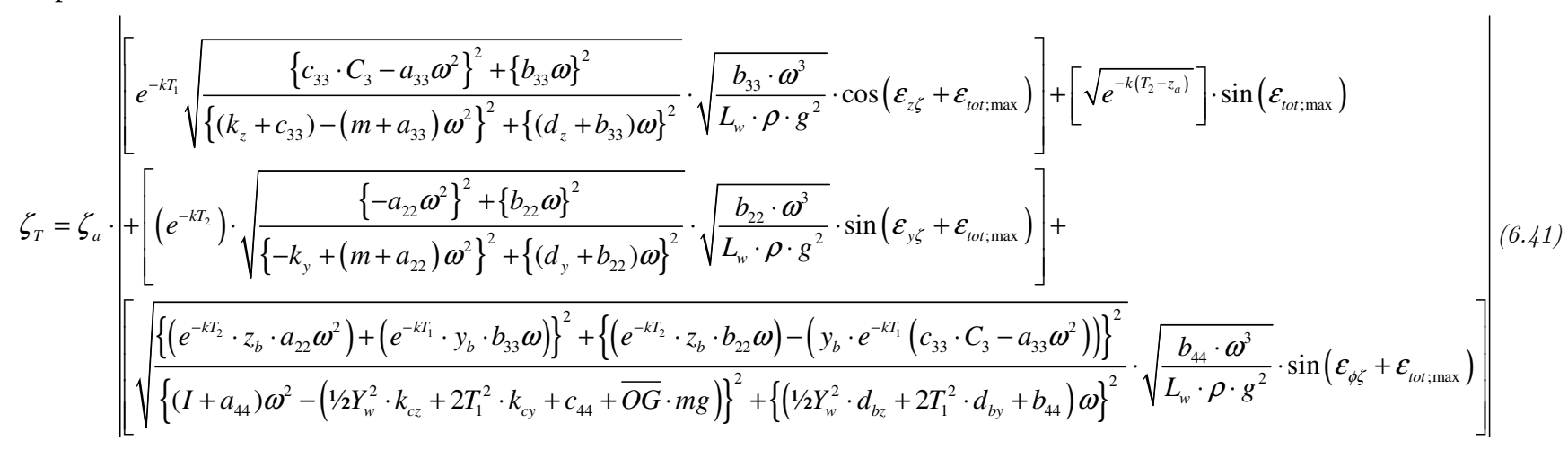

Where:

$\varepsilon_{\text {tot } ; \text { max }}:$ The point where the maximum total transmitted wave amplitude occurs according to appendix B.V.a

This transmitted wave amplitude has to be smaller than the allowed wave amplitude as it was described by equation 6.40 to guarantee $100 \%$ harbour efficiency. 


\subsection{Conclusions}

From equation 6.41 , it can be concluded that the factors that determine the dynamic reaction and thus the radiated wave amplitude are:

$\begin{array}{lll}Y_{w} & : & \text { The structural width } \\ L_{w} & : & \text { The structural length } \\ T_{1} & : & \text { The structural draft } \\ T_{2} & : & \text { The screen draft } \\ m & : & \text { The structural mass } \\ k_{m z} & : & \text { The mooring stiffness } \\ d_{m z} & : & \text { The mooring damping }\end{array}$

With these parameters, an optimal floating breakwater has to be designed in order to meet the requirement of equation 6.39 .

The model, as it is presented in this chapter does not include the energy losses in the zone around the structure. In reality, some of the incident wave energy will be dissipated due to the formation of vortices around the sharp edges of the structure. Another kind of energy loss is the destruction of the path of the wave particles due to the presence of the floating breakwater in the wave domain.

In the following chapter, the influence of the factors on the performance of the floating breakwater has to be determined.

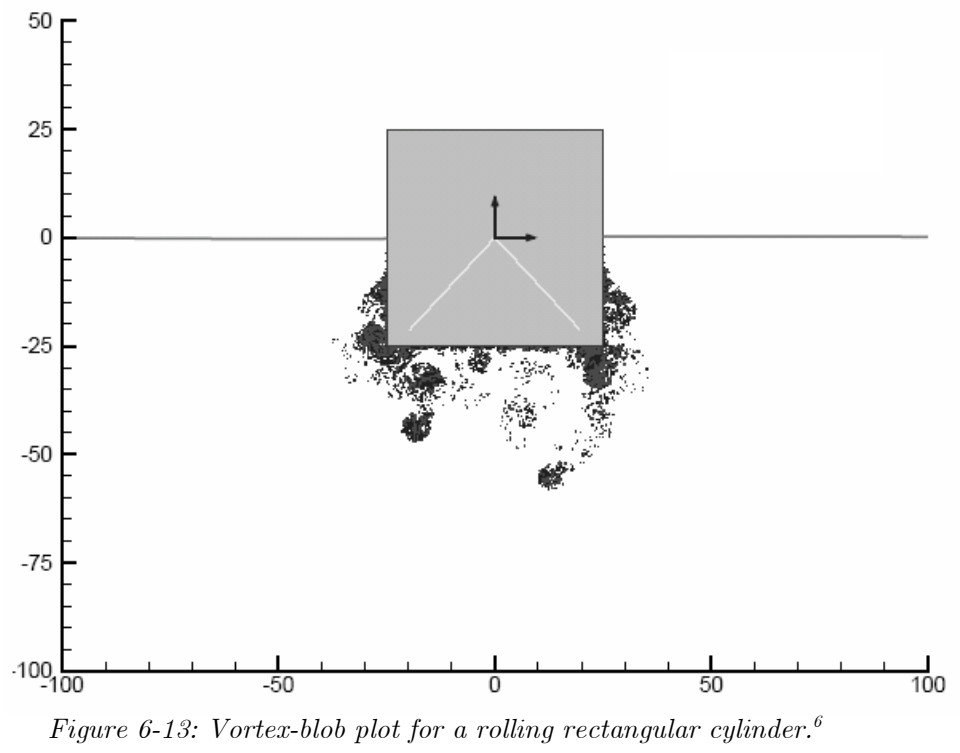

${ }^{6}$ Seah, R.K.M. \& Yeung, R.W. 'Sway and Roll hydrodynamics of cylindrical sections' University of California at Berkeley. 


\section{Chapter 7}

\section{Parameter analysis}

\subsection{Introduction}

The model as it was discussed in the previous chapter will be used to verify the influence of the structural variables on the wave attenuating capacity of the floating breakwater. The three-dimensional potential theory computer model DELFRAC is used to check the results of the dynamic behaviour.

The calculation model and the DELFRAC computations are clarified in Appendix B.

In chapter 7.2 the relations between the structural parameters and the hydrodynamic response is investigated. 26 structures with varying parameters are used for this purpose. These structures are listed in table C-1 of Appendix C. In section 7.3 of this chapter, the influence of the motions and the constructional parameters on the total wave transmission is determined. Since future model tests may alter the calculation parameters as they have been determined in chapter 6 , a sensitivity analysis is performed in section 7.5 to state the consequences when parameters are changed.

Finally, in section 7.4, a structural evaluation is performed where the most important structural parameters are discussed as a starting point of the design process.

The transmitted wave amplitudes and the amplitudes of motions are reflected as dimensionless parameters in this chapter, the so-called Response Amplitude Operator (RAO).

\subsection{Hydrodynamic behaviour}

The results of the calculations, performed for structures S1-S26 are shown in Appendix C.

\subsubsection{Structural width}

The influence of the structural width on the hydrodynamic behaviour depends on the draft and the weight of the structure. The larger the draft, the larger the influence of the horizontal wave force. Since the draft and the structural mass are inextricable connected to one another, two calculations were performed to verify the influence of the structural width. The first calculation is performed with a constant draft, a varying width and thus a varying mass. For the second calculation, the mass was set as a constant and the width and draft are variable.

When the structural width is increased while the draft is kept constant, the mass will increase too. Although the horizontal wave force on the structure will not change, the increase of the mass is the reason why the sway motion amplitude decreases (figure C-1).

A wide and heavy structure is hard to put into a vertical oscillation. Besides the mass another phenomenon affects the vertical amplitude of motion. A wide structure is not able to move along with the relative short-period waves. The wider the structure, the longer the wave period on which the structure will resonate. 
The resonance peak will disappear gradually when the structural width is increased due to the large hydrodynamic damping (figure C-3). The same phenomenon holds for the influence of the structural width on the rotational motion (figure $\mathrm{C}-5$ ).

For structures S6-S10, the mass is set as a constant while the structural width and thus the draft will vary. A remark has to be made on the reliability of the results of structures S6,S7,S9 and S10. As discussed in chapter 6.2 , Vugts did his measurements of the hydrodynamic coefficients in a width/draft $\left(\mathrm{Y}_{\mathrm{w}} / \mathrm{T}_{1}\right)$ range between 2 and 8. Since structures S6 and S7, but S9 and S10 in particular, are out of this range, the reliability of the results for structures S9 and S10 is doubtful.

From figure C-2 it appears that the increase of the width (S6 to S7), and thus a decrease of the draft, results in an increase of the sway motion amplitude. However, a further increase of the structural width (S7 to S8) will result in a decrease of the sway motion amplitude. This phenomenon is due to the equilibrium of the wave exciting forces and the hydromechanical forces and motions.

When de structural width is increased, the draft decreases. Since the horizontal wave exciting force has an exponential distribution into the depth, a small decrease of the draft has limited influence on the total wave exciting force compared to the loss of hydromechanical force. In other words: the amplitude of the motion increases when the decrease of the wave exciting forces is less than the decrease of the hydromechanical forces.

$\frac{d}{d z} F_{w ; y}>\frac{d}{d z} F_{h ; y} \quad \rightarrow$ increase of the sway amplitude

The opposite is true when the structural width is increased further more (S7 to S8):

$\frac{d}{d z} F_{w ; y}<\frac{d}{d z} F_{h ; y} \quad \rightarrow$ decrease of the sway amplitude

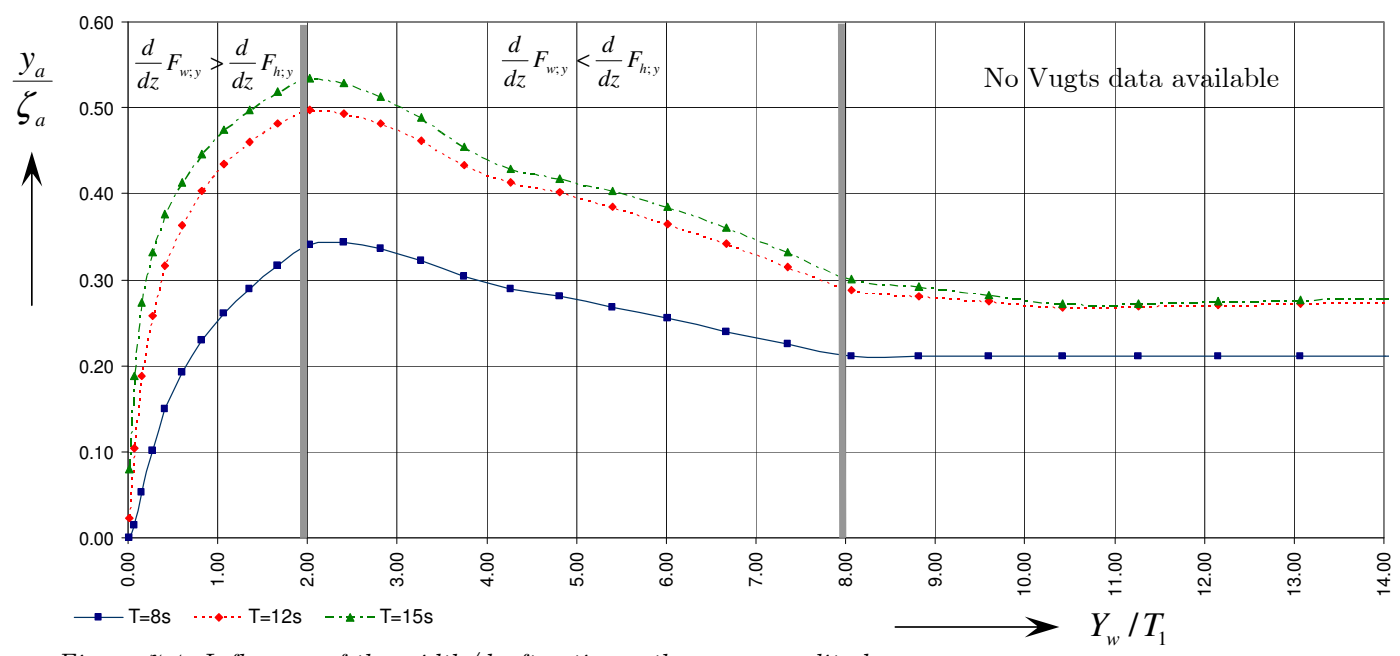

Figure 7-1: Influence of the width/draft ratio on the sway amplitude.

Since the wave energy is exponentially distributed over the depth, structure S6 needs a longer wave to put it into a vertical motion as structures S7-S10 do. The decrease of the resonance peak for structures with a large width, as is shown in figure $\mathrm{C}-4$, is due to the large hydrodynamic damping. The same phenomenon holds for the influence of the structural width on the rotational motion (figure C-6). 


\subsubsection{Draft / Mass}

Appendix C.II.b shows the influence of the draft on the hydrodynamic behaviour of the floating breakwater. The mass automatically changes for a free floating structure when the draft is changed and the width is kept constant.

The influence of the draft on the sway motion amplitude was already discussed in the previous section. The increase of the sway amplitude when the draft is increased and the decrease of the amplitude when the draft is increased further more is clarified with equations 7.1 and 7.2 .

A structure with a large draft will need a longer wave period to put it into a vertical oscillation (figure C-8). Structure S14 for example will hardly show any movement when a 10s wave period is considered. Structures with smaller drafts on the other hand will have their resonance peak in this same period.

Although this seems to be a strange development, the resonance peak grows when a heavier structure is considered (compare S3 with S14). This is due to the decrease of the hydrodynamic coefficients as they were presented in Appendix B. As long as the decrease of the hydrodynamic parameters is larger than the increase of the mass, the resonance peak will grow.

The same phenomenon holds for the roll behaviour. Due to an increasing draft, the momentum on the structure will grow faster than the resistance, resulting in an increasing resonance peak. When the draft is increased even more, this motion will become less due to the exponential wave force distribution.

The larger the draft, the smaller the part of the wave energy that transmits underneath the floating breakwater. Due to the exponential distribution of the wave energy, increasing the draft $(d T / d z)$ further more becomes less effective. An increase of the draft from 5 to 20 meters has more effect on the wave transmission than a further increase from 20 to 40 meters.

\subsubsection{Rigid screen underneath the floating breakwater}

A screen increases the structural draft of the structure and is assumed to be weightless. Due to the presence of a screen, the hydrodynamic coefficients of roll and sway will increase.

The effect of different screen lengths on the sway motion of the structure is shown in figure C-11 of appendix C. The effect of a screen is comparable to the increase of the floater-draft as discussed in the previous section. Since the structural mass will have no influence in this case, the effect of an increasing screen draft on the sway motion mainly depends on the wave period. Small wave periods will have no influence on the sway motion when the screen length is large enough (S18). However, longer waves will cause a larger sway motion amplitude due to the surplus of wave force according to equation 7.1.

The effect of a screen on the roll motion is shown in figure C-12. A screen results in an increase of the hydrodynamic coefficients and thus a decrease of the roll motion amplitude. When the hydrodynamic coefficients change, the dynamic behaviour of the floating breakwater changes as well, resulting into a shift of the resonance peak. The hydrodynamic coefficients are known for a structure with a screen length up to 20 meters, as discussed in Appendix B. Since the screen of structure S18 is out of this range, the result is divergent. Measurements on the hydrodynamic behaviour of floating bodies with screens underneath have to be performed in the future in order to verify the calculation results.

The influence of a screen on the underflow is the same as was discussed in section 7.2.1. A screen will be an effective measure when the increase of the sway transmitted amplitude is less compared to the decrease of the transmitted amplitude due to the underflow. 
As long as the increase of the sway transmitted amplitude due to the increased draft is less than the decrease of the underflow, a screen is recommendable. In other words:

$$
\frac{d \zeta_{T ; \text { sway }}}{d z}<\frac{d \zeta_{T ; \text { underflow }}}{d z} \rightarrow \text { screen recommended }
$$

\subsubsection{Mooring line stiffness}

An increase of the mooring line stiffness puts higher requirements on the local soil conditions.

Although an increase of the mooring line stiffness does not agree with requirement 2 of chapter 5.3 , the effect has to be verified in order to determine the value of the mooring system on the hydrodynamic behaviour of the floating breakwater.

An increase of the mooring line stiffness results in a decrease of the sway motion amplitude (figure C-14). The mooring line stiffness has a more interesting effect on the vertical motion. An initial increase results in a decrease of the heave motion amplitude. When the mooring stiffness becomes large enough, the natural frequency is changed in such a way that an under-damped hydrodynamic system is generated. Resonance on relative high frequency waves is the result of the change in dynamic behaviour. 


\subsubsection{Review}

Sway

$-$

The sway reaction depends on the width-draft-mass relation.

Increasing the mass and width while the draft is kept constant leads to the decrease of the sway motion amplitude.

- $\quad$ An increase of the draft results in an increase of the sway motion amplitude whenever there is a surplus in the wave exciting force relative to the hydromechanical forces. Increasing draft $T_{1}$ has more effect on the sway motion than an increase of the screen draft $\mathrm{T}_{2}$ has. This is due to the own mass that is involved when the draft of the floating part $\left(\mathrm{T}_{1}\right)$ is considered.

\section{Heave}

$-$

$-$

An increase of the structural mass results into a shift and a decrease of the resonance peak.

When the structural width is increased, while the draft is kept constant, the mass increases, resulting into a decrease of the heave motion amplitude. This decrease is due to the increasing mass as well as the increase of the hydrodynamic coefficients.

A wide structure with little draft will move along with the water particles when relative long waves are considered. However, no resonance peak will be visible since the hydrodynamic heave damping is very large for wide structures.

As long as the decrease of the hydrodynamic parameters is larger than the increase of the mass, the resonance peak will grow for structures with a constant width.

The structural draft increases when the mooring lines stiffness is increased. The heave motion decreases when the mooring stiffness gets larger. The resonance peak will shift to the area with higher wave frequencies when the mooring stiffness is increased (fig. 6-4).

Roll

- $\quad$ A wide structure is less vulnerable for roll motion compared to a narrow structure. This is due to the increase of the hydrodynamic parameters.

- $\quad$ Since the hydrodynamic moments are generated by the horizontal (sway) and the vertical (heave) hydrodynamic forces, the roll motion has the same characteristics as the sway motion when the draft is increased and the same characteristics as the heave motion when the structural width is considered.

- $\quad$ The resonance peak of the roll motion is very large. This holds for the calculation model as it is considered in this report as well as the calculation results of DELFRAC. A roll motion as big as $\phi_{a}=1.75[\mathrm{rad}]$ is a reason to doubt on the roll results when the natural frequency is considered. It is a well known phenomenon that DELFRAC roll amplitudes are always too high compared to reality.

\section{Underflow}

- $\quad$ The underflow decreases when the structural draft increases. 


\subsection{Wave Transmission}

The hydrodynamic behaviour of a floating breakwater and the underflow are determined by the dimensions of the structural parameters as has been clarified in the previous section. Although the floating breakwater efficiency is not assessed by the amplitudes of motion, they are an important parameter to control the wave transmission.

In short period waves, the transmitted wave amplitude is dominated by the contributions of underflow, sway and roll. When the wave frequency is near the vertical natural frequency of the floating breakwater, the contribution of heave to the total wave transmission becomes the dominating factor. Waves with a long period will penetrate to the harbour side of the floating breakwater due to the underflow. Between these three regions, where a dominating factor is present, 'boundary regions' are visible (cross-hatched areas) where the wave transmission is minimal. This is due to the fact that the waves, produced by the heave motion in these areas, have a mutual phase difference of $\pi$ in comparison to the waves radiated by other motions and the underflow.

With the structural parameters, as discussed in chapter 7.2, the position of the boundary regions can be changed. In figure 7-2 the boundary regions $\left(\zeta_{T} / \zeta_{a}<0.10\right)$ for a structure with a mass of 153,750 tons and a width of 20 meters are around 9 and 11 seconds. When the structural width is changed to 36 meters while the draft is kept constant, the regions shift to 10.2 and 13 seconds.

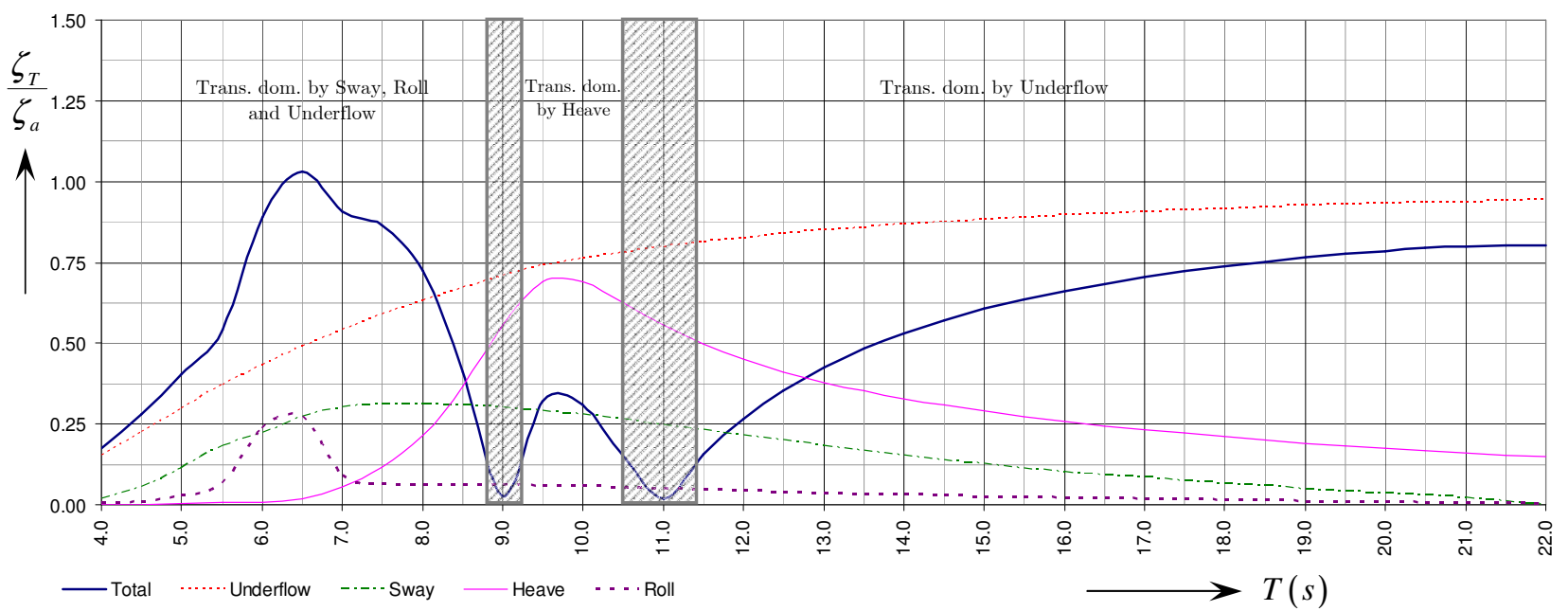

Figure 7-2: Wave transmission for a structure: $Y_{w}=20 \mathrm{~m}, T_{1}=15 \mathrm{~m}, M=153,750 t, T_{2}=0$.

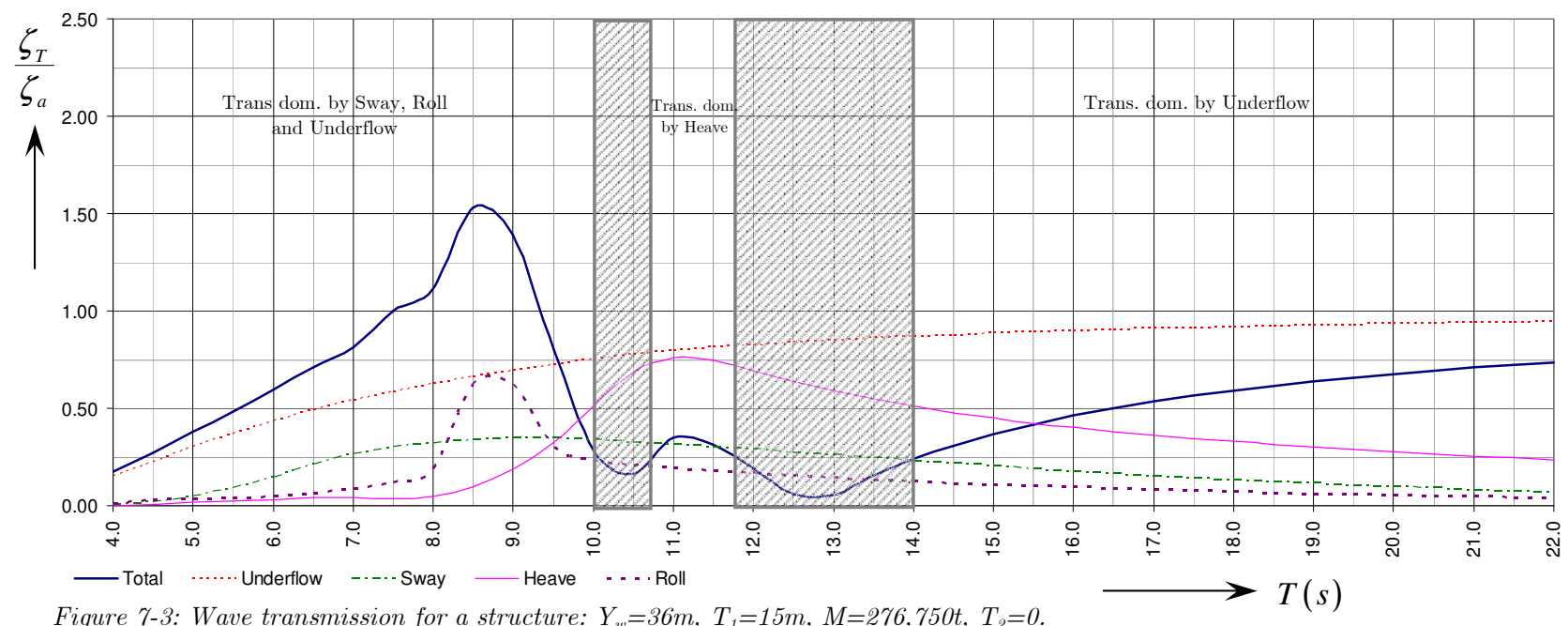


In their report, Yamamoto and Yoshida ${ }^{1}$ concluded that very low transmission occurred at very low or high frequencies with moderate results in between. This conclusion matches the results as they are presented in figures 7-2 and 7-3.

Every wave period requires an adaptation of the structural behaviour and thus the structural lay-out. To state the attenuating capacity of the floating breakwater, three-dimensional design graphs are developed. With these graphs it is possible to verify the influence of a structural change on the efficiency of the floating breakwater.

The three-dimensional design graph contains a wide variety of graphs as they were shown in figures 7-2 and 7-3. Figure 7-4 shows cross-sections of the design graph on $\mathrm{T}=8$ and 13 seconds and $\mathrm{T}_{1}=18$ and $32 \mathrm{~m}$ (no screen).

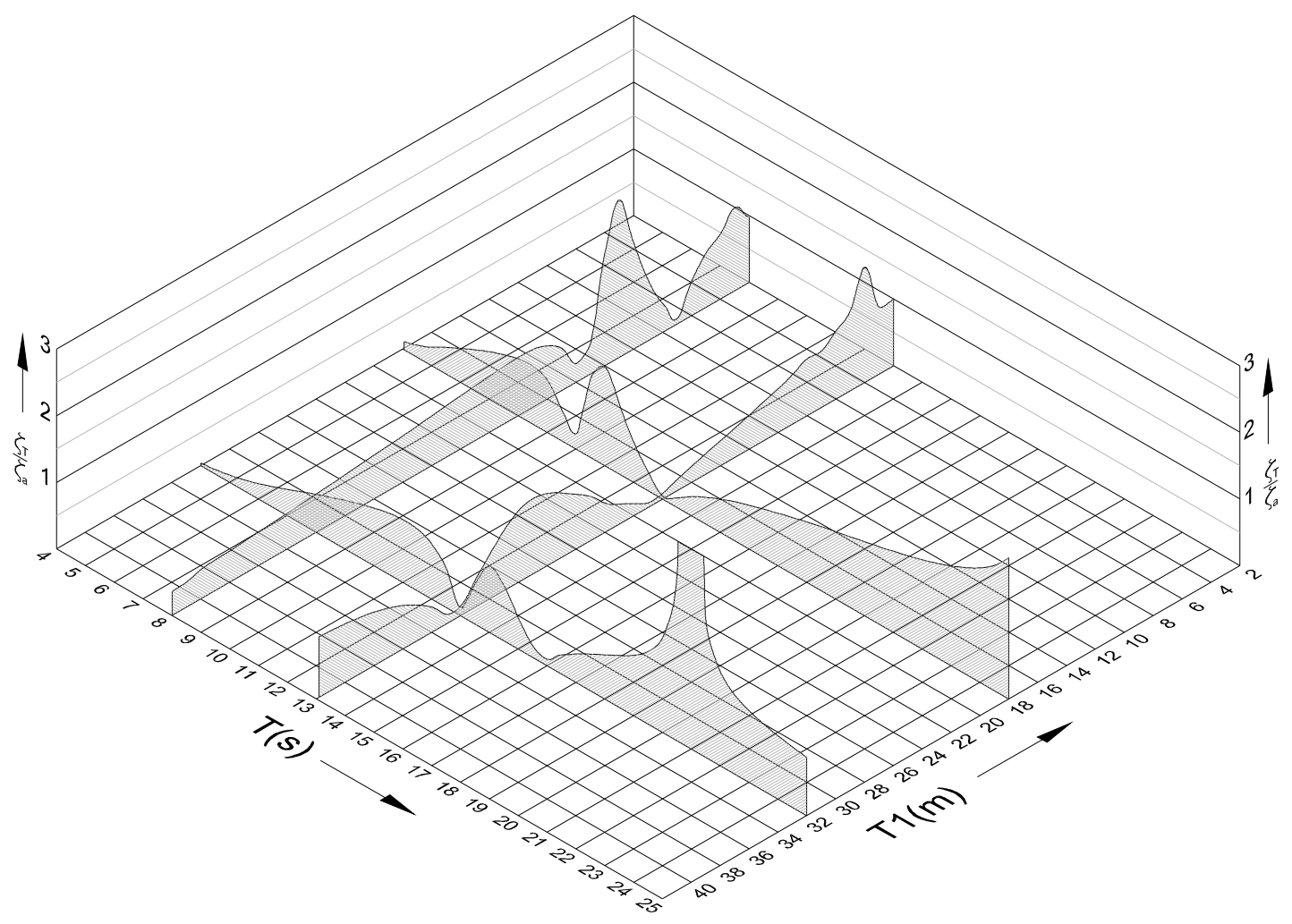

Figure 7-4: Three dimensional over view of the transmitted wave amplitude ratio (RAO) with a varying wave period and draft.

The optimal draft for several wave periods is easily determined with a three-dimensional graph as shown in figure 7-4. When graphs are available for every structural variable, the influence of these variables on the total wave transmission can be visualized.

Figure 7-5 gives a three-dimensional representation of the influence of a certain structural parameter on the attenuating capacity of the floating breakwater. The most interesting areas in this graph are the cross-hatched areas, where wave transmission is minimal (0-10\%). Areas with a larger wave transmission are visible as the red and yellow peaks in figure $7-5$.

The magnitude of the considered structural parameter should be variable in order to stay in the low-transmission area. The variable in figure 7-5 for example has to increase when the wave period increases.

Appendix C.III gives an overview of the period-related wave transmission when different structural parameters are varied. Some areas in these graphs are highlighted and denoted as 'No Vugts data available'.

${ }^{1}$ Yamamoto, T. and Yoshida, A. (1979). 'Large wave tank tests on taut-moored breakwaters'. Coastal structures '79, Vol.II, ASCE. 
In these areas, estimated hydrodynamic coefficients are used based on the Vugts measurements in the width/draft domain: $2 \leq Y_{w} / T_{1} \leq 8$.

Since heave is the dominant factor and the heave hydrodynamic coefficients decrease when the width/draft ratio decreases (appendices B.III.a.1 and B.III.b.1), the calculation results in the domain $0 \leq Y_{w} / T_{1}<2$ are expected to be more accurate than the non-bounded calculation results in the domain $10.4<Y_{w} / T_{1} \Rightarrow \infty$.

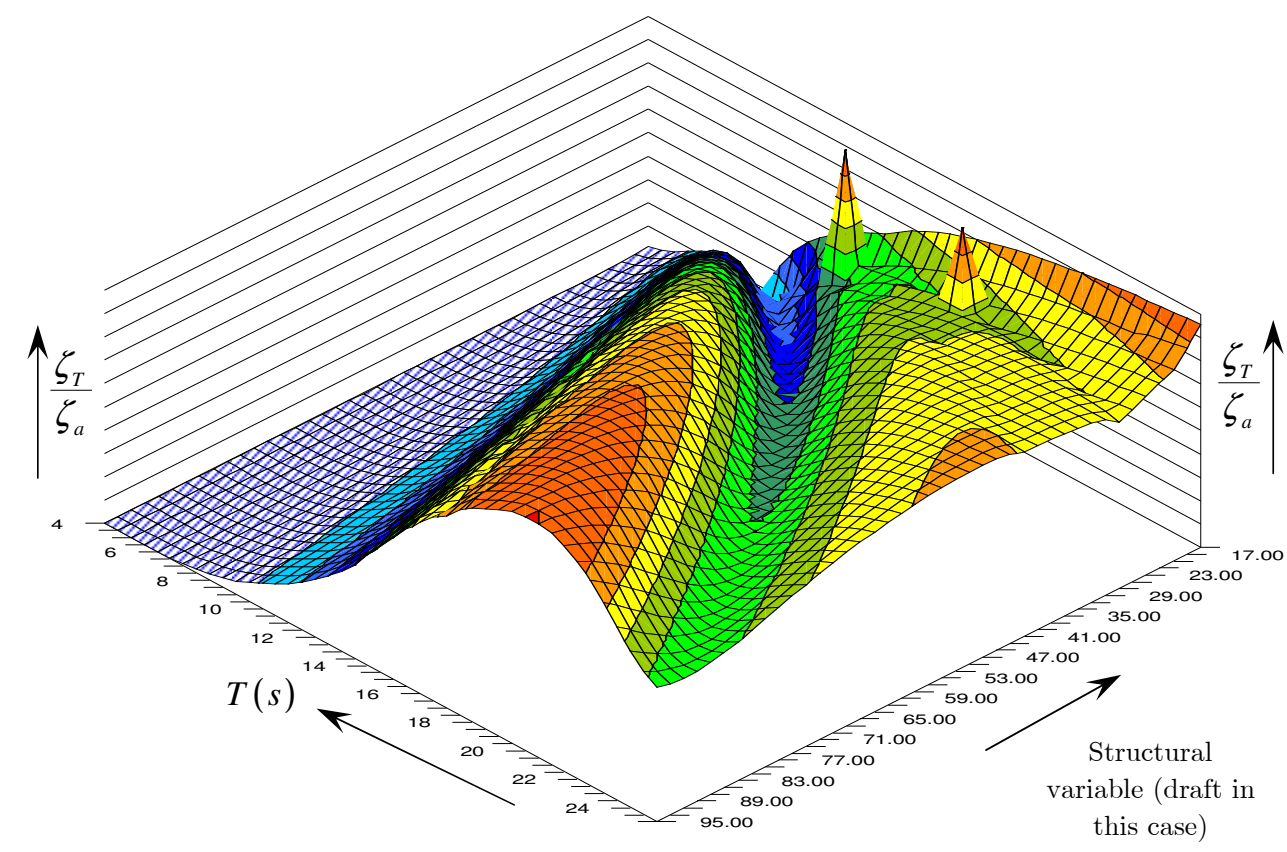

Figure 7-5: Three-dimensional representation of the wave transmission.

The dominance of certain motions on the wave transmission is not clearly visible in the graphs as they are presented in Appendix C.III. Figures that show the influence of the individual motions on the wave transmission are presented at the bottom of the pages. 


\subsubsection{Structural width}

The influence of the width on the wave transmission is visualized in figures C-16 and C-17 of appendix C.III. Best performance is gained when the phase shift of the heave motion is $-1 / 2 \pi$. The radiated wave by the heave motion will in this case have a total phase difference of $\pi$, compared to the wave generated by the other motions and the underflow. In the red areas, the heave motion radiated wave will be in phase with the waves, generated by the other motions and underflow. The performance of the floating breakwater in these red areas is poor since it will act as a wave generator in stead of a wave attenuator.

An increase of the width will move the blue areas, where a maximum wave reduction of $70 \%$ is gained, to the longer wave period range. A wide structure will perform better when longer waves are considered.

The waves generated by the roll motion interrupt the low-transmission area, as shown in figures C-16 and C-17. This is the reason why a minimum wave transmission of $70 \%$ can not be guaranteed for a floating breakwater that is able to extend its width.

From figure C-17 it appears that the calculations, performed in the domain $10.4<Y_{w} / T_{1} \Rightarrow \infty$ can not be trusted. This is due to the fact that the heave hydrodynamic coefficients are kept constant in the calculation model when the width/draft ratio exceeds the value of 10.4. The assumed added mass as well as the damping will be too low and the hydrodynamic behaviour will not be representative any more.

A small structural width and a large draft have a positive influence on the wave transmission coefficient, as visible in figure C-17. A wave reduction of $70 \%$ for waves with periods up to 14 seconds can be realized, as long as the structural draft is kept large enough.

\subsubsection{Draft}

The performance of a floating breakwater with a variable draft is less affected by the radiated waves of the roll motion than structures with a variable width. As is shown in figure C-18 of appendix C.III, structures with a limited draft in high frequency waves have a poor attenuating capacity. When the draft gets too small, the floating breakwater might even become a wave generator. This is the case when the phase of the heave motion is $+1 / 2 \pi$. Waves generated by the heave motion will be in phase with the waves generated by the other phenomena in this case.

Since the increase of the structural width has a positive contribution when high wave frequencies are considered and the increase of the draft becomes a positive factor when longer waves are involved, a combination of these two factors might be a good solution.

When the structural width and the draft are varied with a factor $Y_{w} / T_{1}=1$, the low-transmission area has a continuous course. The blue-coloured area is not interrupted by the waves, generated by the roll motion as was the case when concepts C. $Y_{w 1}$ and C.Y ${ }_{w 2}$ were considered.

An increase of the width/draft ratio results in a shift of the low-transmission area as shown by the dashed line in figure C-19. Although this seems to be a positive behaviour (the floating breakwater is able to be effective in a larger wave period range) the efficiency soon becomes affected by the waves that are generated by the roll motion. The low-transmission area will be interrupted again or will even disappear when width/draft ratios are near the value of 3 .

A decrease of the width/draft ration will shift the low-transmission area as is shown by the dash-dotted line in figure C-19. This shift is due to the reduced wave transmission by the underflow. The amplitude of the generated waves by all three motions will decease to. 


\subsubsection{Screen}

A screen underneath the floating breakwater has some influence when the wave periods are small. When the wave periods get larger, a screen will have a negative effect on the wave attenuating capacity of the floating breakwater. This phenomenon is shown in figure 7-6. The larger the screen draft, the larger the period range where it has a positive effect on the wave transmission. For example: when a screen with a length of $20 \mathrm{~m}$ is applied underneath the structure, it will have a positive effect when wave periods until 12 seconds are considered (fig 7-6). For waves with periods of more than 12 [s], the total wave transmission coefficient of sway and underflow is more than 1 . The screen will in this case behave as a sail and the positive effect of the screen has become a negative factor. Roll is not involved in figure 7-6 since this becomes an important factor when long wave periods are considered. The overall picture for screen lengths up to $40[\mathrm{~m}]$ is shown in figure C-20 of appendix C.III.

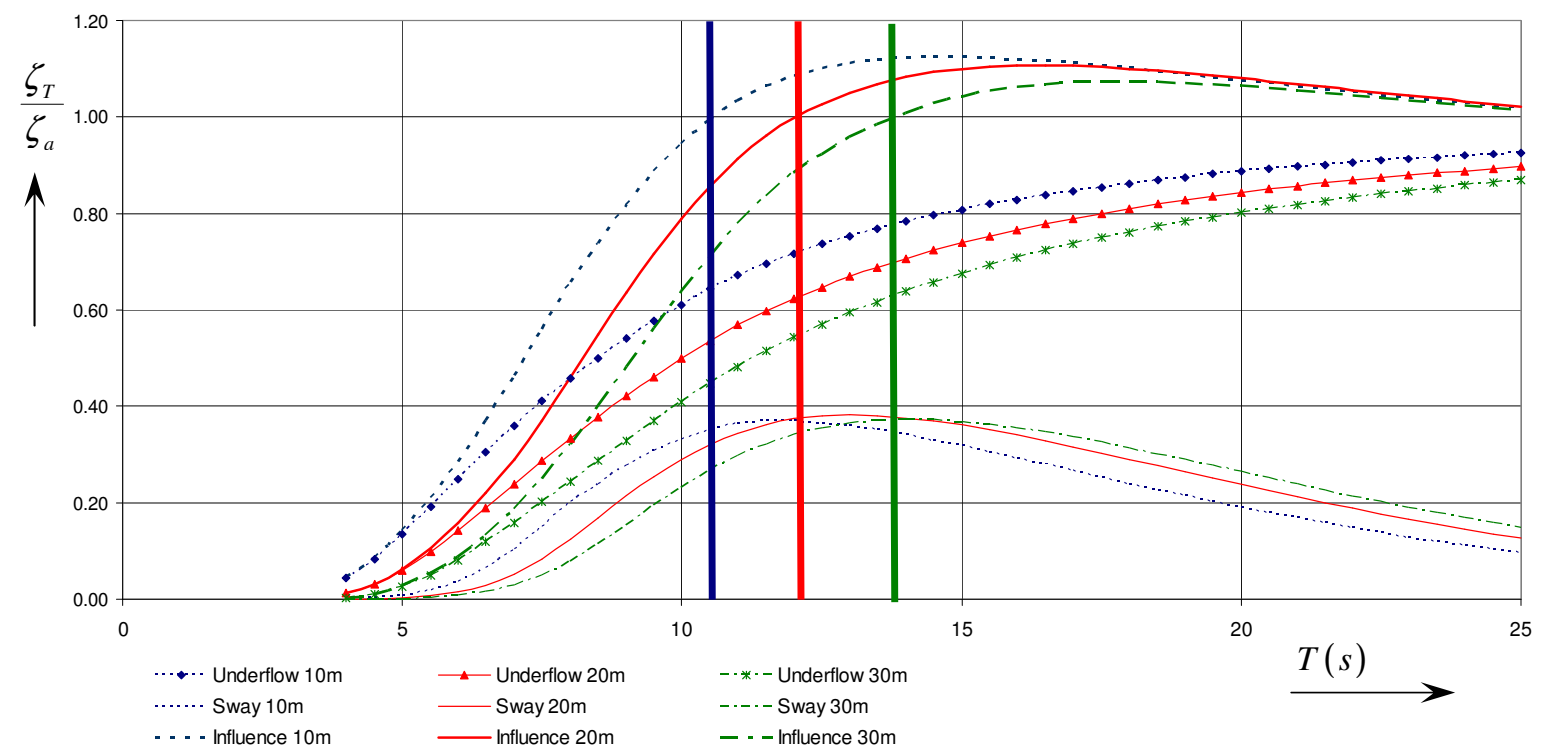

Figure 7-6: Efficiency of a screen underneath the floating breakwater

Due to the assumption that a screen underneath the floating breakwater does not affect the development of the vertical pressure component, the heave motion transmitted wave is not influenced by the screen. This assumption may result in deviations when model tests are conducted.

\subsubsection{Mooring stiffness}

An increase of the vertical mooring line stiffness results in a decrease of the heave motion and thus a decrease of the transmitted wave height, generated by this motion. This statement was already verified in paragraph 7.2.4. Figure C-21 confirms this statement.

All the wave generating factors change when the vertical mooring stiffness is changed. This is due to the fact that an increase of the vertical mooring stiffness results into an increase of the draft. 


\subsection{Optimal floating breakwater design}

The analysis of the influence of the several structural variables on the attenuating performance of the floating breakwater revealed the following:

- $\quad$ The width/draft ratio of floating breakwaters should be large enough when small wave periods are considered in order to maintain the phase shift of $-1 / 2 \pi$ for the heave motion generated wave.

- $\quad$ A decrease of the width/draft ratio is necessary when longer wave periods are considered. When the magnitude of this ratio is kept around 1, limited influence of the roll motion transmitted wave is observed.

- $\quad$ Increasing the draft of the structure with a rigid screen is only valuable when small wave periods are considered. For longer waves, the presence of a screen underneath the floating breakwater might even have a negative influence on the performance.

The optimal floating breakwater performance is gained when the structural appearance can adapt itself on the wave period as shown in figure $7-8$.

The optimal width/draft ratio per wave period that is obtained from figure 7-8 is shown in the figure below. Figure 7-7 does not say anything about the magnitude of the width or the draft, but it shows the importance of the width and the draft for different wave periods. The width for example has to be larger than the draft when small wave periods are considered.

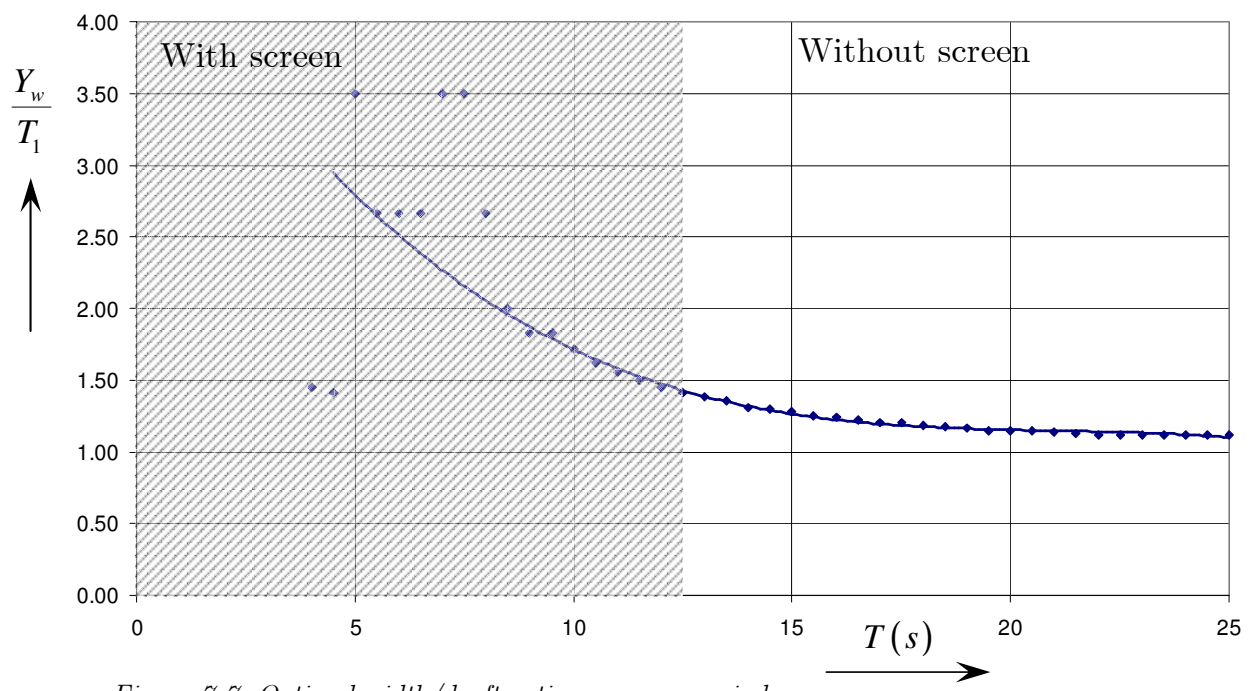

Figure 7-7: Optimal width/draft ratio per wave period.

A change of the width/draft ratio will have the same effects as discussed in section 7.3.2.

Although (in theory) the optimal floating breakwater design has to vary according to figure 7-8, this does not automatically result in optimal performance as well. A breakwater performs optimal when no wave transmission occurs. Since this is a impossible task for a floating breakwater, figure 7-8 reflects how the floating breakwater appearance should vary in order to reach the best possible performance.

For example: when waves with periods of 8 [s] are considered, the structural width should be 18 [m], the draft should be $8[\mathrm{~m}]$ and a screen with a length of $18.50[\mathrm{~m}]$ should be present underneath the structure. A structure with a width of $86[\mathrm{~m}]$ and a draft of $78[\mathrm{~m}]$ will also be effective, but offcourse it will be madness to apply such a structure. 
When waves with periods of $12[\mathrm{~s}]$ are considered, the structural dimensions have to be adapted in order to reach optimal performance. In this case, the width of the structure has to be $34[\mathrm{~m}]$, the draft $24[\mathrm{~m}]$ and a screen with a length of $14.50[\mathrm{~m}]$ will have a positive contribution on the performance.

\begin{tabular}{|c|c|c|c|}
\hline C.optimal & Symbol & Unit & Magnitude \\
\hline Length & $L_{w}$ & $\mathrm{~m}$ & 500 \\
\hline Width & $Y_{w}$ & $\mathrm{~m}$ & Variable \\
\hline Draft & $T_{1}$ & $\mathrm{~m}$ & Variable \\
\hline Screen draft & $T_{2}$ & $\mathrm{~m}$ & Variable \\
\hline Mass & $M$ & tons & Variable \\
\hline Mooring stiffness & $k_{m}$ & $t / s^{2}$ & 0 \\
\hline
\end{tabular}

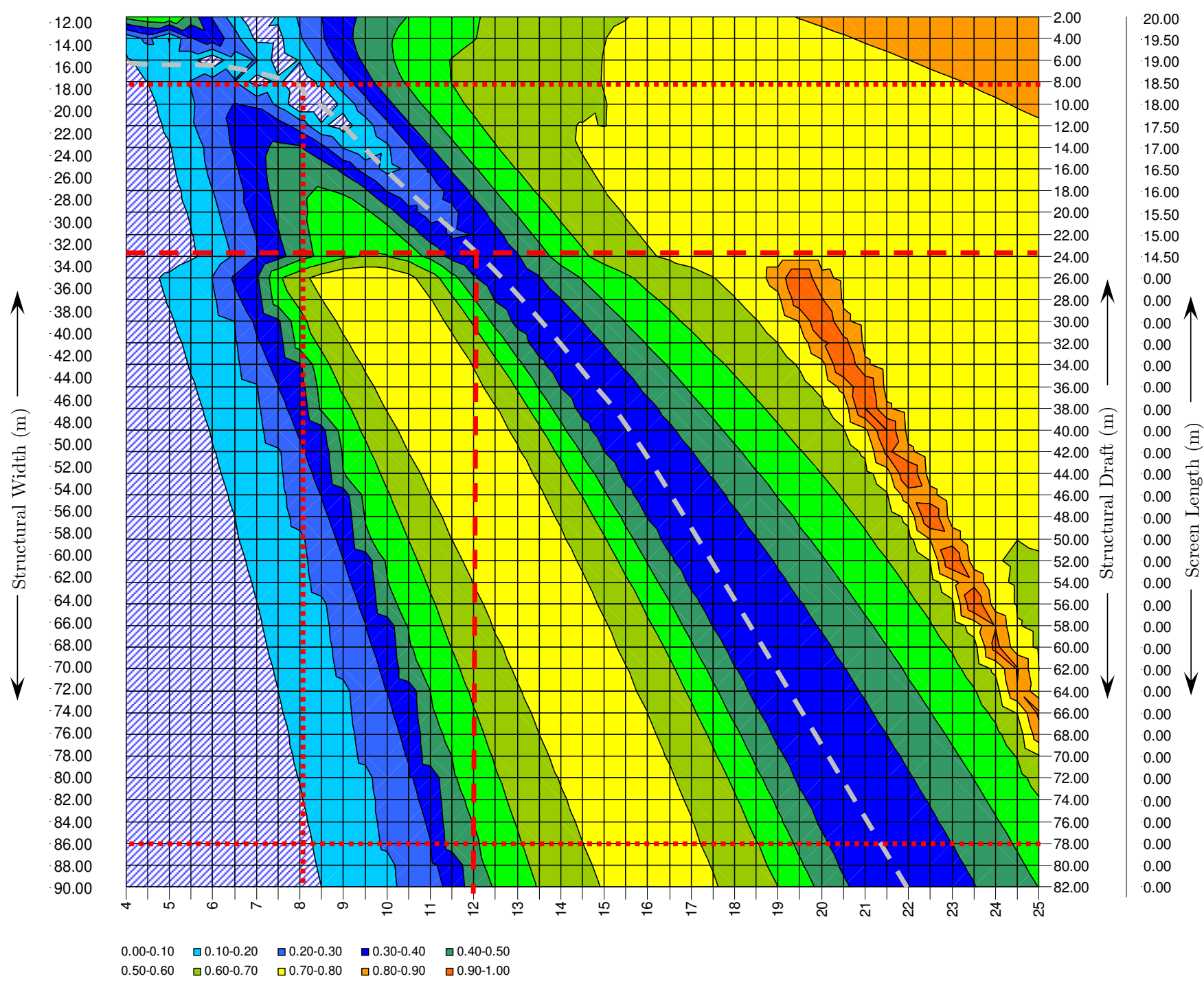

Figure 7-8: Optimally performing floating breakwater.

A floating breakwater is able to diminish the incoming wave amplitude for $95-100 \%$ when wave periods smaller than 9 seconds are considered. Waves with periods between 9 and 10.5 seconds are attenuated for 80-90\%. The floating breakwater is able to diminish the incoming wave amplitude for waves with periods between 10.5 and 13 seconds for $65-80 \%$. Waves with periods larger than 13 seconds can be diminished by $65-70 \%$. 


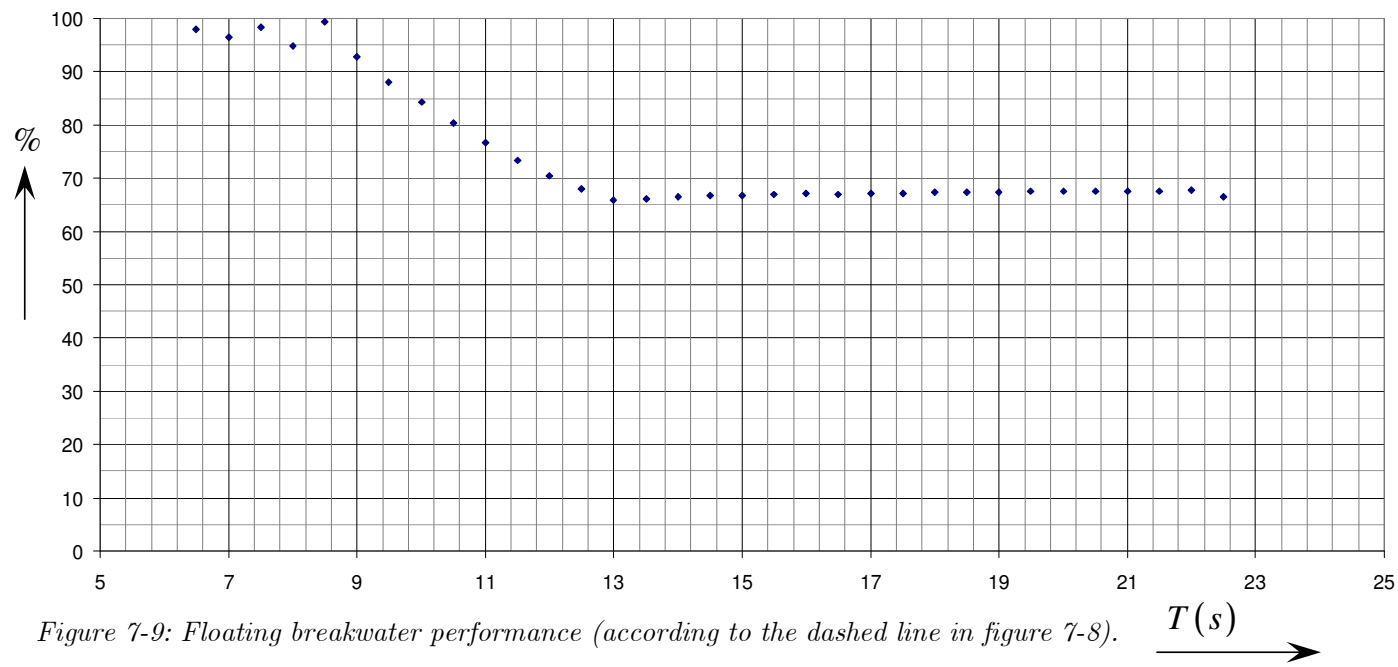

When this performance is coupled to the maximum allowed ship motions, as they were discussed in chapter $5,100 \%$ harbour efficiency for waves with periods larger than 13 seconds can only be guaranteed when the wave height does not exceed 2.45 metres.

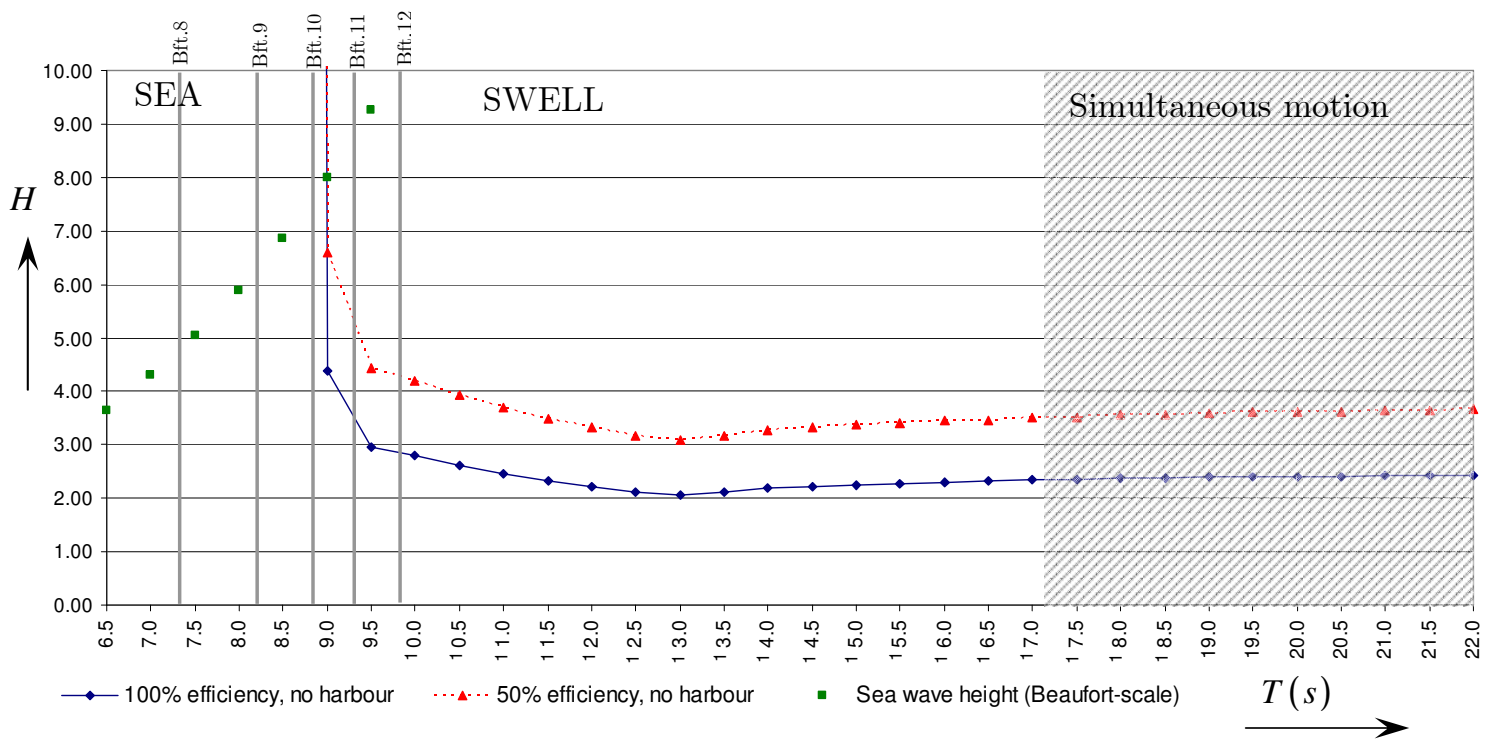

Figure 7-10: Maximum allowed incoming wave heights for a 6000 TEU container vessel.

Ali $(2005)^{2}$ proved that the working efficiency is not affected for wave amplitudes up to 3 meters when the floating harbour itself is used as a wave barrier. In this case, the vessels are positioned at the leeward side of the floating harbour. Ali used the JONSWAP wave spectrum, coupled to the Beaufort scale (table 5-4), to determine the harbour efficiency in various weather conditions.

Surge was denied by Ali since the amplitudes of surge are around 2 meters in North Sea conditions. Although the transmission coefficient is larger for surge, the transmitted amplitudes are smaller due to the flattened character of the wave amplitudes of surge.

When Beaufort scale 10 is considered, the mean zero-crossing wave period ( $\mathrm{T}_{2}$ in table 6-2) is $8.85[\mathrm{~s}]$ and the significant wave height is $7.45[\mathrm{~m}]$ in coastal areas similar to the North Sea. In open-Ocean areas, this period becomes $8.80[\mathrm{~s}]$ and the significant wave height $7.45[\mathrm{~m}]$ (Bretschneider). The RAO of the heave motion of the FTCT-feeder system, based on the calculations performed by Ali, are shown in figure 7-11.

${ }^{2}$ Ali, A. (2005). Msc thesis 'Floating Transshipment Container Terminal', Delft University of Technology. 
Besides the RAO of the FTCT-feeder system, the transmission coefficient of the floating breakwater in the Beaufort-wave period range is shown in figure 7-11 as well.

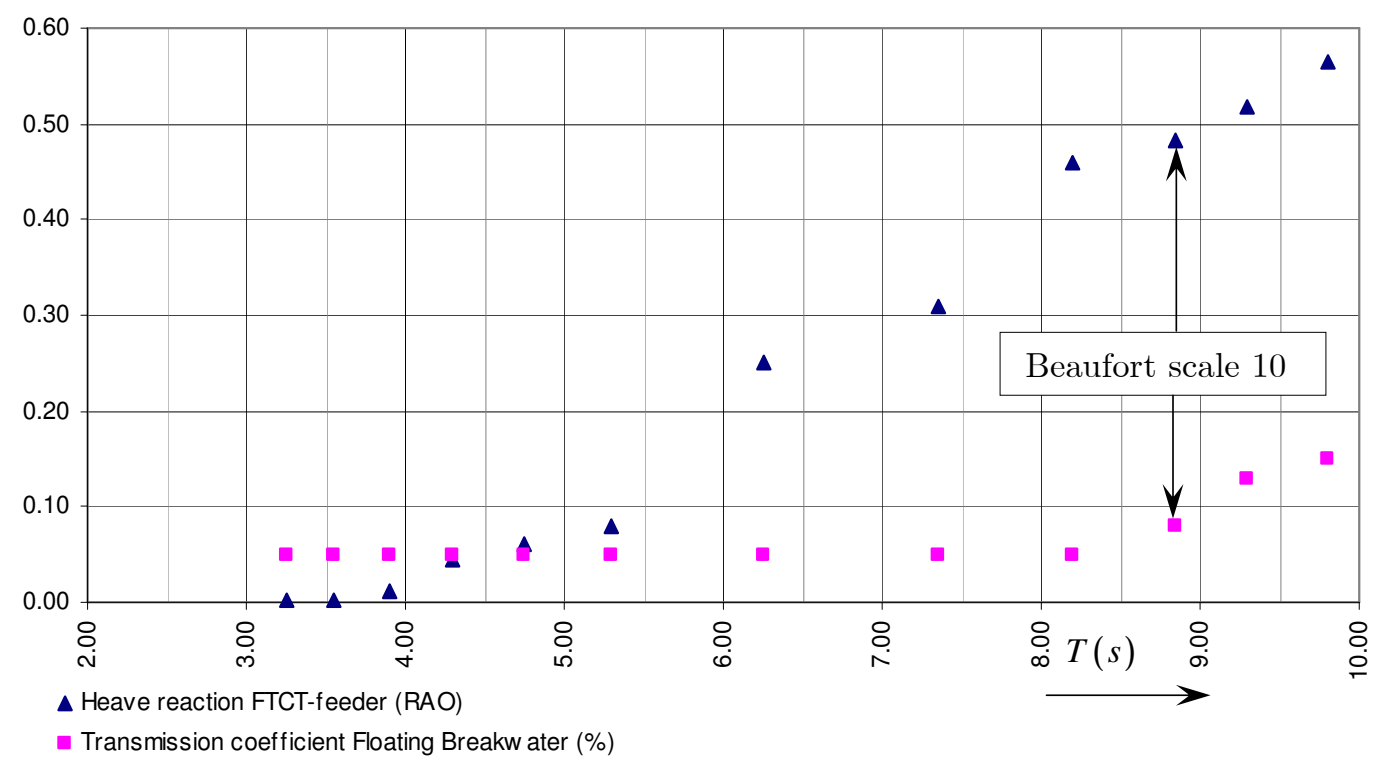

Figure 7-11: Heave reaction of the FTCT-feeder system and wave transmission of the floating breakwater.

Although the efficiency of any container harbour might be affected by the wind when a storm of Beaufort scale 10 is considered, the mutual motions of the ship and the FTCT will not be the cause of this inefficiency when a floating breakwater is present.

$\mathrm{Ali}^{3}$ calculated that the relative heave motion between the FTCT and the moored feeder vessel will have significant amplitudes of about $1.80[\mathrm{~m}]$ when the significant wave height of Beaufort scale 10 is considered. Since the significant wave amplitude in these weather conditions is $3.73[\mathrm{~m}]$, the relative heave RAO of the FTCT-feeder system becomes:

$\frac{z_{a}}{\zeta_{a}}=\frac{1.80}{3.73} \approx 0.48$

For optimal harbour efficiency, the heave motion amplitude $\left(\mathrm{z}_{\mathrm{a}}\right)$ may not exceed $0.40[\mathrm{~m}]$ (paragraph 5.2.1). In other words: the maximum allowed wave amplitude, able to pass the floating breakwater is $0.83[\mathrm{~m}]$. With the knowledge that the optimized floating breakwater (theoretically) is able to attenuate $92 \%$ of the incoming wave amplitude for waves with a period of $8.80[\mathrm{~s}]$ (figure 8-8), the incoming wave amplitude may not exceed:

$\zeta_{a} \leq \frac{0.83}{0.08}=10.42[m]$

A floating harbour, protected by a floating breakwater is now within the design conditions until Beaufort scale $11\left(\mathrm{H}_{1 / 3}=8.70[\mathrm{~m}], \mathrm{T}_{2}=9.30[\mathrm{~s}]\right)$. This is the case when the influence of swell is neglected. Waves with periods longer than $10[\mathrm{~s}]$ are most of the times swell. Since swell in areas similar to the North Sea has wave heights around $2[\mathrm{~m}]$ the floating breakwater reduces these waves enough to guarantee $100 \%$ harbour efficiency.

${ }^{3}$ Ali, A. (2005). Msc thesis 'Floating Transshipment Container Terminal', Delft University of Technology. 


\subsection{Sensitivity analysis}

The objective of a sensitivity- or uncertainty analysis is to get an idea of the influence of calculation parameters that have been qualified as being uncertain in this stage of the research on the wave transmission. It is important to understand the behaviour of the hydrodynamic system when future (model)tests change the magnitude of the calculation parameters.

The sensitivity analyses are performed for the heave hydrodynamic added mass and damping. Heave is the most important factor determining the attenuating performance of the floating breakwater. Since the heave hydrodynamic added mass and damping are extrapolated from the model tests performed by Vugts (1970), and the magnitude of these factors coincide with a certain uncertainty, they are interesting parameters for a sensitivity analysis. The heave added mass and damping are varied with $10 \%$.

The influence of a change in added mass on the transmission coefficient $\left(\mathrm{C}_{\mathrm{T}}\right)$ is listed in table $7-1$ for different wave periods and width/draft ratios. Figure D-3 in appendix D shows the effect of the added mass graphically.

\begin{tabular}{|c|c|c|c|c|c|c|c|c|c|c|c|c|}
\hline \multirow{2}{*}{$\begin{array}{c}\text { Wave } \\
\text { Period } \\
(\mathrm{s})\end{array}$} & \multicolumn{3}{|c|}{$\mathrm{C}_{\mathrm{T}}\left(Y_{w} / T_{1}=2\right)$} & \multicolumn{3}{|c|}{$\mathrm{C}_{\mathrm{T}}\left(Y_{w} / T_{1}=4\right)$} & \multicolumn{3}{|c|}{$\mathrm{C}_{\mathrm{T}}\left(Y_{w} / T_{1}=8\right)$} & \multicolumn{3}{|c|}{$\mathrm{C}_{\mathrm{T}}\left(Y_{w} / T_{1}=10\right)$} \\
\hline & $-10 \%$ & $\begin{array}{c}\text { Mean } \\
(100 \%)\end{array}$ & $+10 \%$ & $-10 \%$ & $\begin{array}{c}\text { Mean } \\
(100 \%)\end{array}$ & $+10 \%$ & $-10 \%$ & $\begin{array}{c}\text { Mean } \\
(100 \%)\end{array}$ & $+10 \%$ & $-10 \%$ & $\begin{array}{c}\text { Mean } \\
(100 \%)\end{array}$ & $+10 \%$ \\
\hline 6 & 0.38 & 0.41 & 0.47 & 0.60 & 0.69 & 1.36 & 1.58 & 1.64 & 1.70 & 1.68 & 1.76 & 1.83 \\
\hline 8 & 0.71 & 0.70 & 0.69 & 1.23 & 1.17 & 1.13 & 0.60 & 0.65 & 2.27 & 0.56 & 2.31 & 2.26 \\
\hline 10 & 0.79 & 0.79 & 0.79 & 0.59 & 0.58 & 0.57 & 0.28 & 0.28 & 0.28 & 0.23 & 0.24 & 0.25 \\
\hline 12 & 0.84 & 0.84 & 0.83 & 0.66 & 0.65 & 0.65 & 0.36 & 0.35 & 0.35 & 0.27 & 0.27 & 0.27 \\
\hline 14 & 0.92 & 0.92 & 0.91 & 0.71 & 0.71 & 0.71 & 0.47 & 0.47 & 0.46 & 0.38 & 0.38 & 0.37 \\
\hline 16 & 0.85 & 0.85 & 0.85 & 0.76 & 0.76 & 0.76 & 0.56 & 0.56 & 0.56 & 0.48 & 0.48 & 0.47 \\
\hline
\end{tabular}

Table 7-1: Variation of the heave hydrodynamic added mass.

A change of the added mass results into a horizontal as well as a vertical shift of the transmission peaks, visible in the short-wave period range. This behaviour is predictable according to the theory, as explained in chapter 6.1.2. The deviation seems to disappear when longer wave periods are considered. Since the added mass is one of the factors that determine the natural frequency of the system, a change will especially be noticed around the natural period. This phenomenon is visualized in figure $7-12$. The consequences of a $10 \%$ increase or reduction of the added mass are shown for various width/draft ratios.

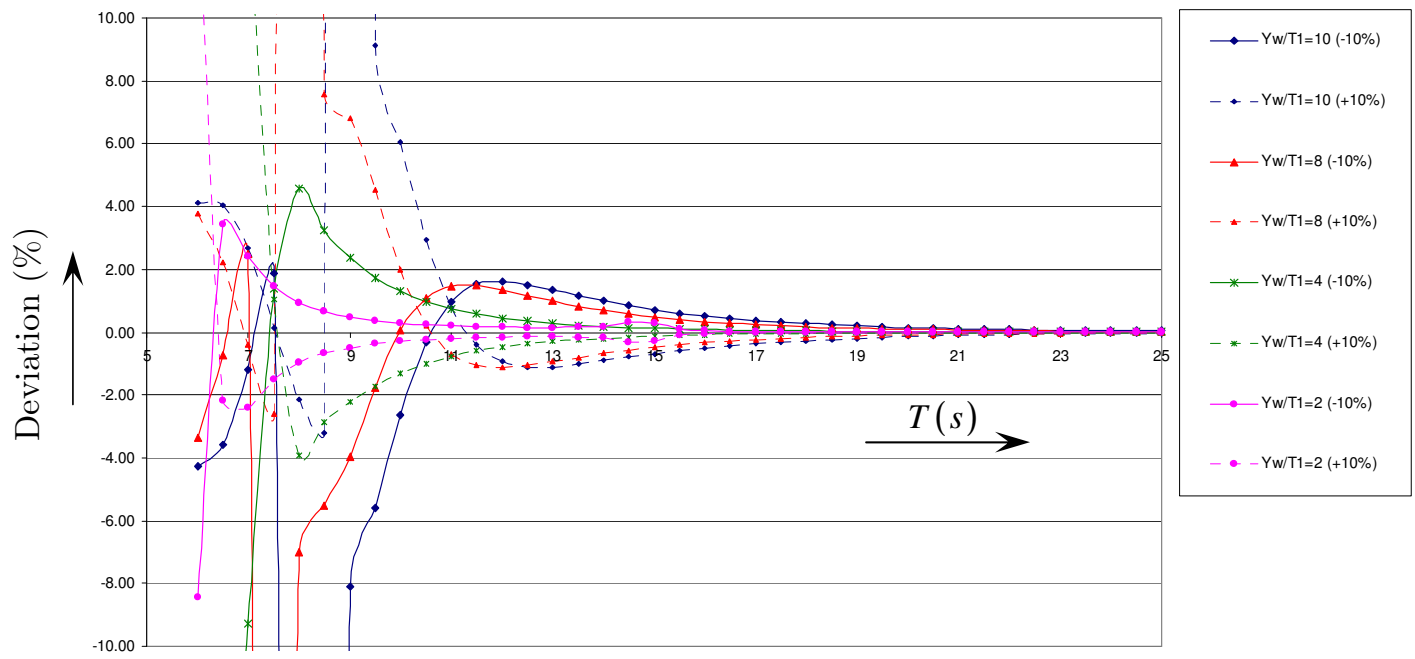

Figure 7-12: Deviation of the transmission coefficient when a standard deviation of 10\% is applied for the heave hydrodynamic added mass. 
A realistic representation of the added mass is crucial in order to be able to determine the exact location of the low-transmission area.

A $\pm 10 \%$ change of the hydrodynamic damping will result in deviations, as listed in table 7-2. A graphical representation of these values is figure D-4 of appendix D.

\begin{tabular}{|c|c|c|c|c|c|c|c|c|c|c|c|c|}
\hline \multirow{2}{*}{$\begin{array}{c}\text { Wave } \\
\text { Period } \\
\text { (s) }\end{array}$} & \multicolumn{3}{|c|}{$\mathrm{C}_{\mathrm{T}}\left(Y_{w} / T_{1}=2\right)$} & \multicolumn{3}{|c|}{$\mathrm{C}_{\mathrm{T}}\left(Y_{w} / T_{1}=4\right)$} & \multicolumn{3}{|c|}{$\mathrm{C}_{\mathrm{T}}\left(Y_{w} / T_{1}=8\right)$} & \multicolumn{3}{|c|}{$\mathrm{C}_{\mathrm{T}}\left(Y_{w} / T_{1}=10\right)$} \\
\hline & $-10 \%$ & $\begin{array}{c}\text { Mean } \\
(100 \%)\end{array}$ & $+10 \%$ & $-10 \%$ & $\begin{array}{c}\text { Mean } \\
(100 \%)\end{array}$ & $+10 \%$ & $-10 \%$ & $\begin{array}{c}\text { Mean } \\
(100 \%)\end{array}$ & $+10 \%$ & $-10 \%$ & $\begin{array}{l}\text { Mean } \\
(100 \%)\end{array}$ & $+10 \%$ \\
\hline 6 & 0.39 & 0.41 & 0.43 & 0.71 & 0.69 & 0.66 & 1.59 & 1.64 & 1.69 & 1.71 & 1.76 & 1.80 \\
\hline 8 & 0.72 & 0.70 & 0.68 & 1.15 & 1.17 & 1.20 & 0.64 & 0.65 & 0.65 & 2.20 & 2.31 & 2.42 \\
\hline 10 & 0.80 & 0.79 & 0.78 & 0.60 & 0.58 & 0.56 & 0.29 & 0.28 & 0.26 & 0.25 & 0.24 & 0.23 \\
\hline 12 & 0.85 & 0.84 & 0.83 & 0.67 & 0.65 & 0.64 & 0.38 & 0.35 & 0.33 & 0.29 & 0.27 & 0.25 \\
\hline 14 & 0.92 & 0.92 & 0.91 & 0.73 & 0.71 & 0.70 & 0.49 & 0.47 & 0.44 & 0.40 & 0.38 & 0.35 \\
\hline 16 & 0.86 & 0.85 & 0.84 & 0.72 & 0.76 & 0.75 & 0.58 & 0.56 & 0.54 & 0.50 & 0.48 & 0.45 \\
\hline
\end{tabular}

A change of the hydrodynamic damping will result in a change in height of the resonance peak. In chapter 6.1.2 it was already proved that damping has an enormous influence on the hydrodynamic behaviour when frequencies close to the natural frequency are considered. The hydrodynamic damping is also a very important parameter to determine the transmitted wave amplitude (eq. 6.41).

The consequences of a $10 \%$ increase or reduction of the hydrodynamic damping are shown in figure 7-13 for various width/draft ratios.

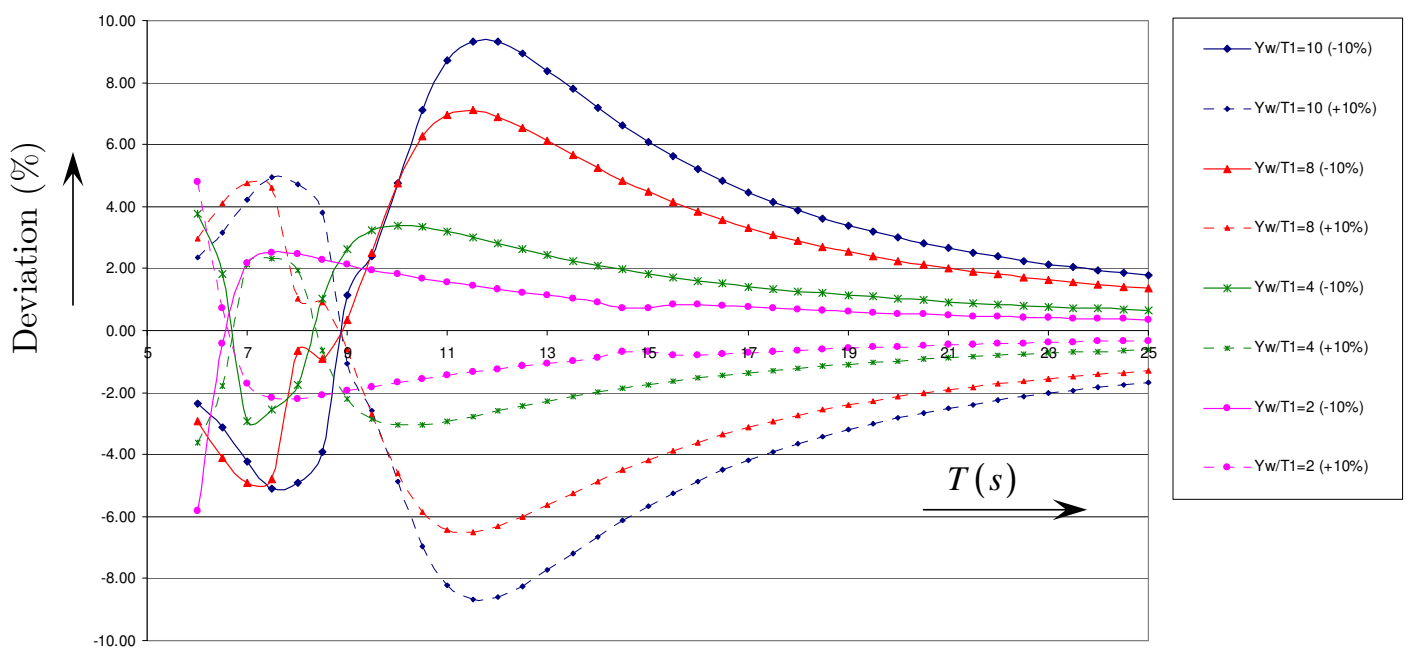

Figure 7-13: Deviation of the transmission coefficient when a standard deviation of $10 \%$ is applied for the heave hydrodynamic damping.

A correct determination of the hydrodynamic damping is essential to be able to verify the performance of the floating breakwater. When the damping is assumed too low, the positive characteristics of the heave motion and thus the attenuating capacity of the floating breakwater are over estimated. 


\subsection{Conclusions}

In this chapter, the influence of several structural variables on the hydrodynamic behaviour and thus the wave attenuating capacity was investigated. A floating breakwater performs best when the following aspects are involved in the design process:

- $\quad$ A structure with a width/draft ratio larger than 2 is needed when wave periods smaller than 10 [s] are considered. The mass as well as the draft of the structure must be chosen in such a way that the performance of the breakwater is optimal, and can stay optimal when wave periods get larger. In other words: the structural appearance for small period waves must be chosen in such a way that the low-transmission area is not disturbed when wave periods get larger.

- A screen underneath the structure is only an effective measure when small period waves are concerned. For wave periods larger than about 10 [s], a screen might even become a transmission amplifier.

- $\quad$ The width-draft-mass relation must be optimized for every wave period. This relation is optimal when the waves, generated by the heave, have a phase difference of $-1 / 2 \pi$ and an amplitude which is large enough to diminish the transmitted waves, generated by the roll, sway and underflow.

Constructive aspects may alter the results as they are presented in Appendix C.III. The mass distribution is assumed to be uniform in the calculation model. This assumption is not true for most of the floating structures from a stability point of view. Extra calculations have to be performed when the structural design is known in order to verify the roll influence on the total wave transmission.

Since Ali (2005) assumed that the oscillating heave motion of the vessel always has a phase difference of $\pi$, compared to the heave motion of the FTCT, he added the heave motion of the vessel and the FTCT to get the total heave motion of the system. This assumption might hold for short period waves, but for longer waves a negative representation of the real situation is obtained.

According to Ali, unprotected floating harbours are able to be efficient until Beaufort scale 6. Above this scale, mutual motions of feeder and FTCT become too large. Since Ali distinguishes a breakwater and a harbour side in the design of the FTCT, less than half of the outline of the FTCT can be used for berthing.

Wind speeds of 24.5 knots ( $44 \mathrm{~km} / \mathrm{hr}$ ) occur when Beaufort scale 6 is considered. With these wind speeds the lifting operations are hindered severely, even when fixed berths are considered. One may conclude that a floating breakwater is thus an unnecessary addition, since the floating harbour it self can cope well enough with the incoming waves related to this wind scale. In chapter 1, second sentence, a harbour is described as a haven for ships. No captain of any ship will enter a harbour in bad weather conditions when the wave conditions inside the harbour are of such a magnitude that damage of the ship can occur due to collisions with the berthing facility. The advantages of an FTCT, protected by a floating breakwater are thus:

- $\quad$ Until Beaufort scale 10, the full berthing capacity of the floating harbour can be used.

- $\quad$ The floating harbour is a safe haven for ships even when weather conditions become bad.

The position of the low-transmission area (fig.8-5) is sensitive to the magnitude of the hydrodynamic added mass. The wave transmission coefficient is affected by the hydrodynamic damping. It is important to know the values of the heave added mass as well as the damping in order to be able to give an optimal prediction of the performance as well as the design of the floating breakwater. 


\section{Chapter 8}

\section{Structural feasibility}

\subsection{Introduction}

In theory, a floating breakwater with the structural requirements as they were determined in chapter 8 will have the best attenuating performance. In practice however it will be very difficult to create a structure that is able to increase its size and weight nine times.

Besides the requirements on the performance, the floating breakwater has to meet the requirements as they were set in chapter 5 as well.

In this chapter an initial design evaluation is performed in order to state the feasibility of a structure with the structural variability as described in chapter 7.4. As an example, a floating breakwater design study is performed for the harbour of Durban (S.A.). Durban is a typical example where a floating harbour could be the solution for the existing local problems of harbour expansion.

\subsection{Requirements and structural restrictions}

The requirements as they were listed in chapter 5.3 can now be specified.

\section{Ad.1 Attenuate waves of the critical wave period range.}

Optimal floating breakwater performance is gained when the structure is able to adapt its appearance on the prevailing wave period. The most important structural parameters affecting the hydrodynamic behaviour of the floating breakwater are:

- $\quad$ Structural width

- $\quad$ Structural draft

- $\quad$ Screen draft

The variance rate of these parameters depends on the critical wave period range present on the project site. Wave periods affecting the floating harbour efficiency are within this critical wave period range and have to be attenuated until an acceptable level.

In the design stage, one has to keep in mind that the calculations have been performed with the following assumptions:

The structure is assumed to be completely stiff in all directions. When this is not the case, the hydrodynamic behaviour will change. It is important to realise that especially the vertical structural stiffness is an important parameter which determines the performance of the floating breakwater. If the bottom of the structure is able to deform, the heave reaction will become less 
and thus the appearance of the design graphs of appendix C will change significantly. Since the sway and the roll contribute negative to the wave transmission, the decrease of these motions will have a positive effect on the wave transmission coefficient.

- $\quad$ Since the influence of the mooring line stiffness is beyond the scope of this thesis, the results may alter when the influence is proved to be different compared to the assumptions that are used in this report.

- $\quad$ The wave periods have been treated separately to determine the performance of the floating breakwater as discussed in chapter 5 . In real Ocean conditions, several wave periods are present at the same moment in time.

\section{Ad.2 Applicable on several locations in several conditions.}

When the floating breakwater is able to perform well in a wide wave spectrum, due to the change in structural appearance as discussed in Ad.1, it is possible to apply the Floating Transshipment Container Terminal (FTCT) at several locations with different wave conditions.

A structure with a stiff mooring system will not perform as well as a free floating breakwater does, as proved in chapter 7.3.4. Since a tension leg mooring system puts high demands on the local soil conditions, it is a positive characteristic that such a system is not necessary.

\section{Ad.3 Manageable, transportable.}

The floating breakwater has to be transported to the location of the FTCT. Besides transportation, it must be possible to rearrange the floating breakwater system to allow changes in lay-out of the floating harbour. Hence it is a possible characteristic that the anchoring system of the floating breakwater does not have to be as complicated as it would be when a tension leg mooring was considered.

\section{Ad.4 Reusable}

A floating breakwater that meets the requirements of Ad.1-3 automatically will be reusable at several locations where different wave conditions are present.

\section{Ad.5 Durable}

A floating breakwater with variable structural parameters, as it is needed according to the calculations results of chapter 7, is the opposite of what an ideal structure should look like in a marine environment. In marine environments, moving and rotational structural elements will soon be affected by biological and chemical elements such as marine life and the salt water.

These elements can not be neglected in the design process of a floating breakwater that is supposed to be adaptable in width, draft and weight. The maintainability is therefore an important aspect during the whole design process. 


\subsection{Case-study}

Until this moment, unlimited structural dimensions were assumed to be possible. From a structural point of view this will result in ridiculous structural dimensions with a beam width that has to vary from 16 to 90 meters and a draft that has to vary between 6 and 82 meters (Appendix C.III).

In realistic situations, it will not be necessary for a floating breakwater to vary its structural dimensions in such a scale. This is due to the fact that a large wave period- and amplitude domain is bounded in real Ocean conditions.

The floating harbour will be located at areas several miles from the shore where near-shore conditions are to be considered. Since wave statistics are available for almost every part of Ocean in the world, the model as it is presented in this report can be used as a design tool to verify the necessary floating breakwater dimensions at specific locations.

As an example, the East-African coast will be discussed. Deep water conditions are encountered in this region and placing floating harbours can be a serious option at locations where this is necessary from an economical as well a technical point of view.

For the East-African coastline, an FTCT is an attractive alternative at the Gulf of Aden and in front of the port of Durban (SA). The wave statistics of the ocean area near Durban will be used in this chapter to develop the optimal floating breakwater for this region.

The case study as it is performed in this chapter will serve as an example on how to deal with the design tool as it was presented in the previous chapter. Once again, one has to realise that the results of the case study, as they are presented in this chapter are still far from reality due to the following arguments:

- $\quad$ The design tool uses regular sinus-shaped waves. In ocean conditions, a more irregular character of the wave period as well as the wave amplitudes is observed.

- $\quad$ Beam-on waves are assumed in the calculation model. However, the prevailing wave direction may alter with tens of degrees in real situations.

- Local conditions may alter the results drastically. For the Durban case, the final design will have to deal with so-called freak waves, since this is a common phenomenon along the East-African coast. 


\subsubsection{The Harbour of Durban}

The Durban Container Terminal (DCT) is the largest container terminal on the African continent. It has six berths and is able to handle 1.2 million TEU per year. Some projections forecast container traffic at the Durban harbour to grow by 4 to 5 percent a year. By 2020, Durban could be handling 2.4 million 20-foot equivalent units (TEU).

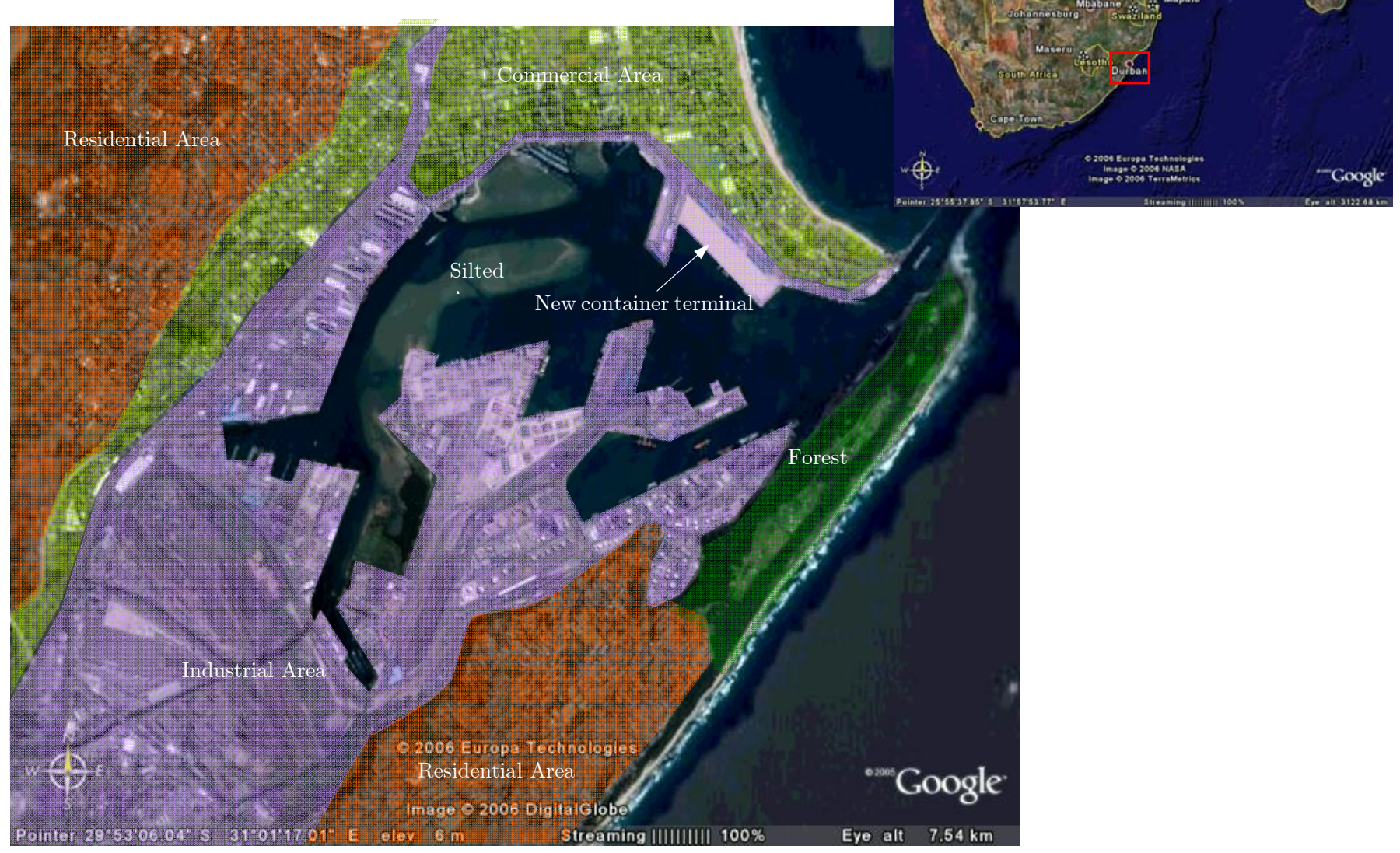

Figure 8-1: The harbour of Durban (South Africa)

An increase of the efficiency of the cargo handling could raise the container traffic further to 5 million TEU by 2020. With such a container handling increase rate, a second container terminal is inevitable. It is unclear where a second container handling facility will be built, as there is limited scope for expansion of the existing Durban harbour, which is hemmed by the city and industrial areas. A second problem is the continuous silt of the existing harbour. Figure 8-1 gives an overview of the Durban harbour area, the adjacent areas and the silted areas inside the harbour area.

Seaward expansion of the harbour is a problem at this location. The coastline is very steep and near shore depths of more than 100 meters are very common in this region.

The Agulhas Current is the main cause for this steep coastal zone. The Agulhas Current, the strong western boundary current of the Indian Ocean, flows southward along the east coast of South Africa. Due to the Agulhas Current and the coastal shape north of Durban, an opposite current occurs as shown in figure 8-2. A seaward expansion does influence the coastal equilibrium in the area. Since the coastline north of Durban is an intensive inhabited area with buildings sometimes at a distance of 50 meters from shore, a change in coastline is not allowed here. 

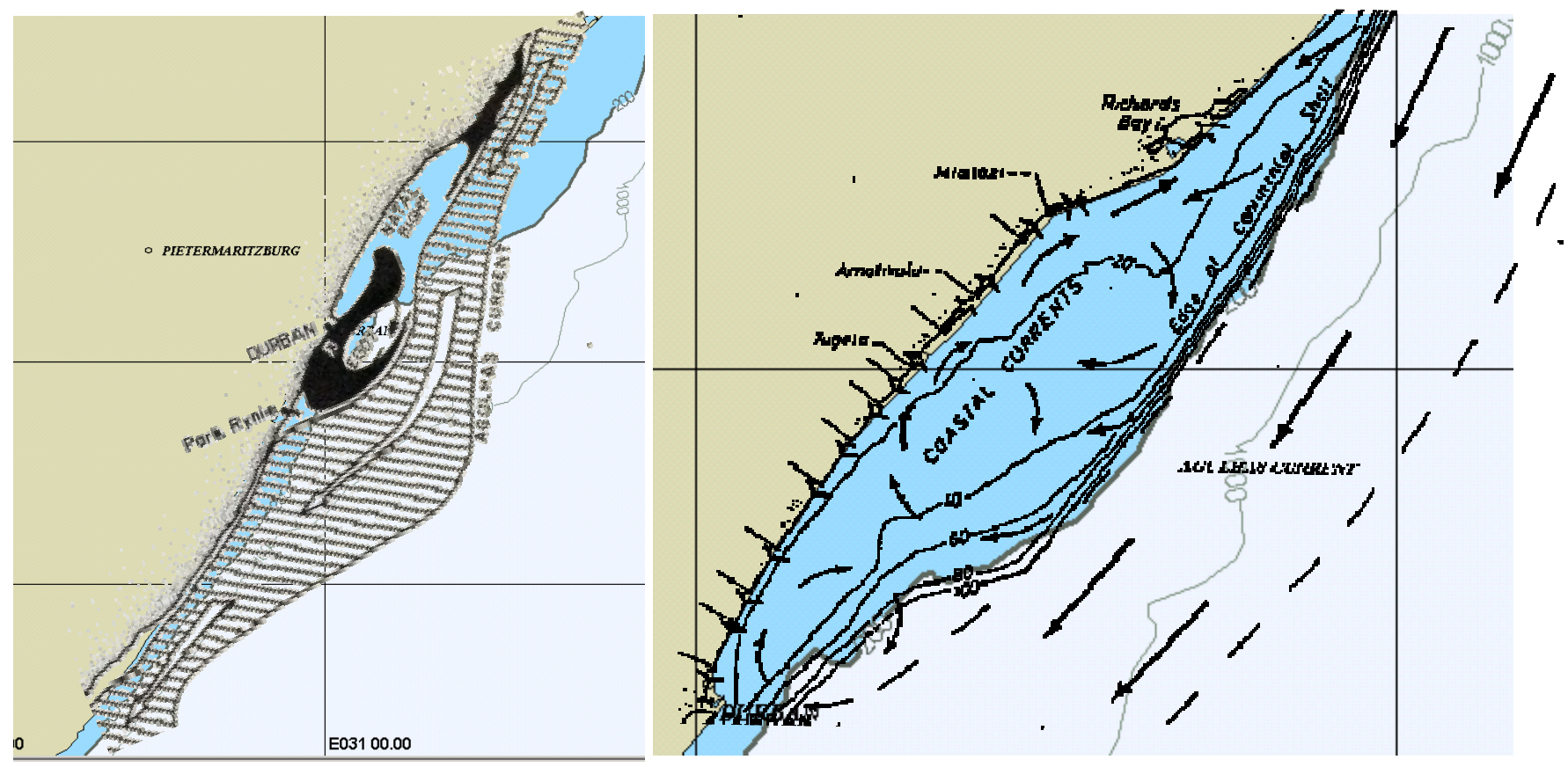

Figure 8-2: Near-shore currents north of Durban.

Since floating structures have very limited influence on the current regime, a FTCT could be the ideal solution to enable the port of Durban to enlarge its container handling capacity and to serve larger vessels. The largest ship that can be served inside the existing harbour has a length of $243.8 \mathrm{~m}$, a maximum width of $35 \mathrm{~m}$ and a draught of $11.9 \mathrm{~m}$, or $12.2 \mathrm{~m}$ according to tide. Larger vessels are common and ships up to $300 \mathrm{~m}$ length and $37 \mathrm{~m}$ beam are regular callers at the outer anchorage off Durban.

\subsubsection{Wave conditions}

The wave conditions in the Durban region have to be analyzed in order to be able to determine the optimal floating breakwater configuration. The combination of wave height and wave period provides the boundary condition to determine which part of the design graphs (appendix $\mathrm{C}$ ) has to be used.

The Global Wave Statistics were used to determine the conditions of the Indian Ocean at the east coast of South Africa. The Global Wave Statistics present an overview of visual obtained data, collected by ships at given times, everywhere on the world oceans. The data that are used in this section are all collected in area 90, which is the part of the Indian Ocean at the east coast of South Africa.

More reliable results are obtained when local wave measurements are used. Since this case study serves only as an example on how to deal with the floating breakwater design when specific locations are considered, a detailed wave spectrum is not necessary in this stage of the research.

The wave statistics as they are used during this case study are summarized in appendix E. Wave periods up to $13[\mathrm{~s}]$ and maximum wave heights of $9[\mathrm{~m}]$ are to be expected in this area. The majority of the waves have periods in the domain 4-10 [s] and amplitudes of 0.5-4.5 [m].

The significant wave height, obtained from the global wave statistics are shown in figure 8-3. The chance that this significant wave height is exceeded is determined per wave period and reflected in figure 8-3 as well. Wave conditions with heights larger than $6[\mathrm{~m}]$ and amplitudes larger than 10 seconds are rare at this location and are expected to occur for $3 \%$ of the time. 


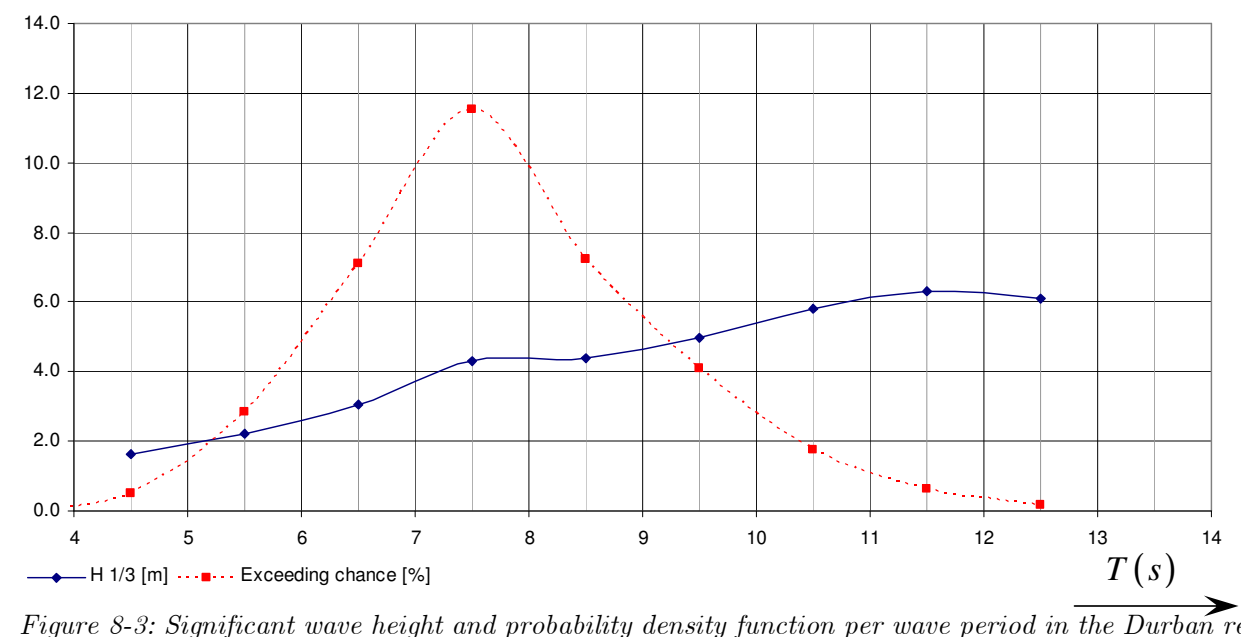

Figure 8-3: Significant wave height and probability density function per wave period in $\overrightarrow{\text { the Durban region. }}$

\subsubsection{Floating breakwater dimensions}

The floating breakwater must be able to attenuate waves in a 3-14 [s] period range. Wave periods until 17 seconds may be expected with a probability of exceedance of 2 [\%] per year (appendix E). The floating breakwater dimensions were determined per wave period with the calculation model. Optimal floating breakwater performance will be obtained when the structure is able to adapt its appearance according to the dashed line in figure 8-4.

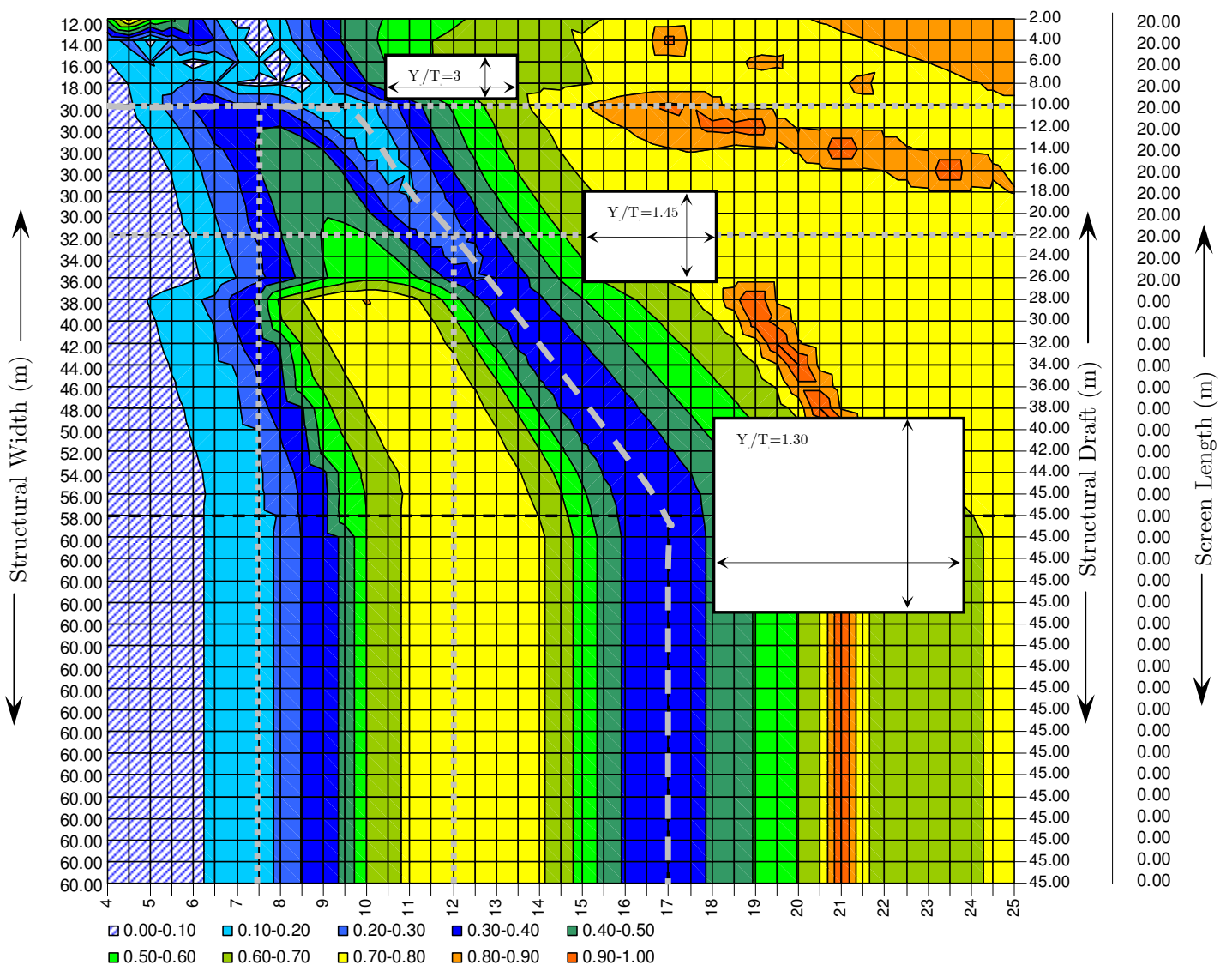

Figure 8-4: Optimal floating breakwater design graph for the 
The structural cross sections are also visible in figure 8-4. The floating breakwater must be able to increase its beam three times and its draft 4.5 times. The structural dimensions of the floating structure for different wave periods as well as the expected attenuating performance by the calculation model are listed in the table below.

\begin{tabular}{c|c|c|c|c}
\hline $\begin{array}{c}\text { Wave period } \\
(\mathbf{s})\end{array}$ & $\begin{array}{c}\text { Width } \mathbf{Y}_{\mathbf{w}} \\
(\mathbf{m})\end{array}$ & $\begin{array}{c}\text { Draft } \mathbf{T}_{\mathbf{1}} \\
\mathbf{( m )}\end{array}$ & $\begin{array}{c}\text { Screen length } \\
(\mathbf{m})\end{array}$ & $\begin{array}{c}\text { Transmission } \\
\text { coefficient } \\
\left(\mathbf{C}_{\mathbf{T}}\right)\end{array}$ \\
\hline$\leq 9.50$ & 30 & 10 & 20 & $0-9$ \\
\hline 10 & 30 & 12 & 20 & 12 \\
\hline 11 & 30 & 18 & 20 & 18 \\
\hline 12 & 32 & 22 & 20 & 25 \\
\hline 13 & 36 & 26 & 20 & 30 \\
\hline 14 & 42 & 32 & 0 & 33 \\
\hline 15 & 46 & 36 & 0 & 33 \\
\hline 16 & 52 & 42 & 0 & 34 \\
\hline 17 & 56 & 45 & 0 & \\
\hline
\end{tabular}

Table 8-1: Optimal Floating Breakwater lay-out and performance for several wave periods.

The transmission coefficients as they are presented in table 8-1 do only involve the influence of the floating breakwater. The transmission coefficient at the leeward side of the FTCT will be even smaller due to the attenuating capacity of this structure. The attenuating capacity of the FTCT is only known for wave periods until 10 [s] since Ali(2005) did not involve surge in his calculations.

The floating breakwater itself will allow the FTCT to be operational for $100 \%$ when wave periods smaller than $9[\mathrm{~s}]$ are present. The efficiency of the berthing facilities at the so-called breakwater side of the FTCT will be affected when wave heights get larger than $2.42[\mathrm{~m}]$ for waves with periods larger than $9[\mathrm{~s}]$.

When the performance of the floating breakwater, as it is presented in figure 9-5 for a 6000 TEU vessel, is coupled to the wave statistics as they are presented in appendix E, it can be concluded that the berths at the breakwater side of the FTCT can be used with an efficiency of $100 \%$ for $95 \%$ of the incoming waves.

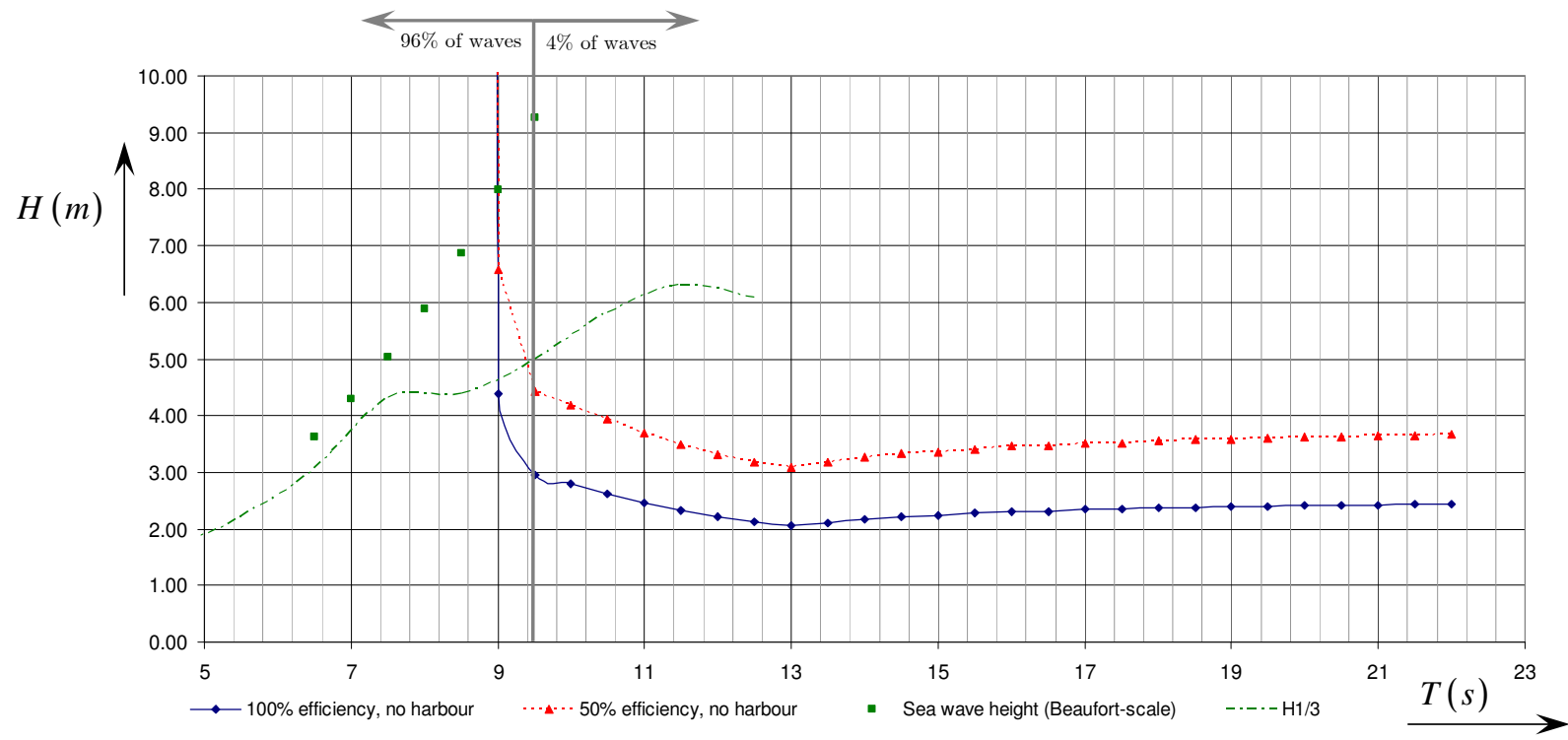

Figure 8-5: Significant wave height and maximum allowed wave height for a 6000 TEU container vessel. 


\subsubsection{The design}

To meet the requirements of Ad.1 and Ad.2, the structural parameters have to vary as showed in figure 9-4. The structure can be transported very easily when the smallest constructional lay-out is considered which has a width of $30[\mathrm{~m}]$ and a draft of $10[\mathrm{~m}]$.

The structure has to increase in draft as well as mass when wave periods get larger. Since the floating breakwater is located at deep-water conditions, the only 'ballast-material' that is available in large quantities is water. Thus the floating breakwater must have ballast tanks that can be filled and emptied again with sea water. These ballast tanks may not be taken too large to avoid the structure

The bottom of the structure is assumed to be stiff in the calculations. This means that the structure should have a stiff, rigid plate at the bottom of the structure that is able to change in width.

Figure 8-6 shows the enormous increase of the structural cross section between 9.5 and 17 seconds when the calculation model is used as a design tool.
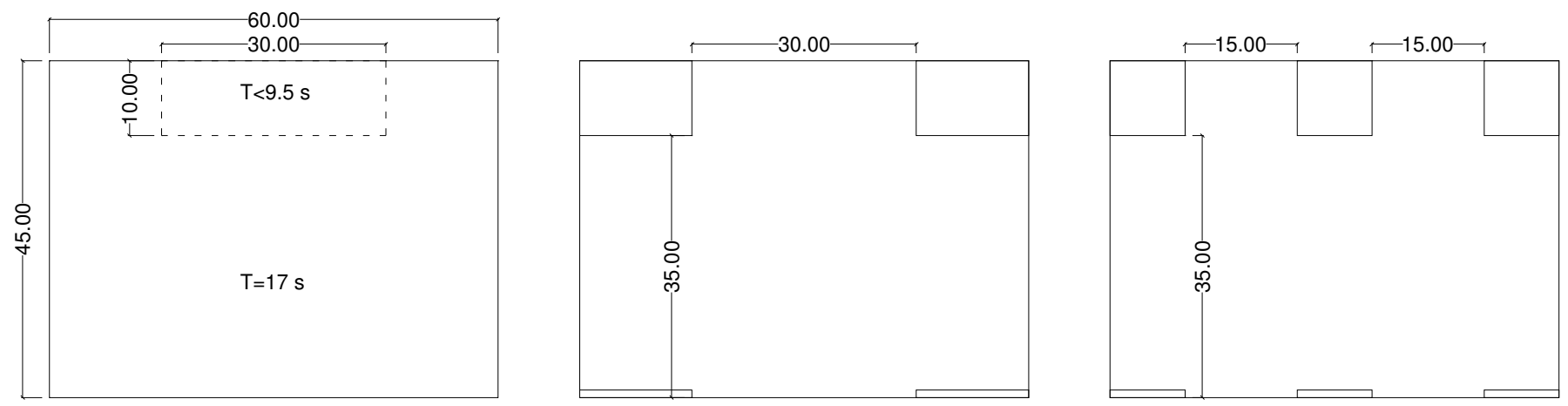

Figure 8-6: Increase of the structural cross section between wave periods of 9.5 and 17 [s].

The increase in width as well as draft is a problem, as shown in figure 8-6. When waves of 17 seconds are considered, only $11 \%$ of the cross section consists of original cross-sectional parts as they were present for waves with periods smaller than $9.50[\mathrm{~s}] .89 \%$ of the cross section consists of water that must be enclosed by the structure in order to use the mass.

To make the structure less vulnerable for the marine environment, moving, rotating and driving parts of the structure should be above water as much as possible.

A possible solution that was obtained from a design study is shown on the following pages. The system as it is shown here has a so-called water cushion system that is able to increase the width as well as the draft of the structure.

- $\quad$ The structural draft is increased by pushing a plate, connected to the water cushions, away from the floating element by vertical piles. The water cushions will become too flexible in the vertical direction when this plate is not present.

- $\quad$ The bottom plate must be permeable at both ends since it may not influence the hydrodynamic behaviour when the total width of the structure is not needed.

- $\quad$ For small-period waves, a screen is visible underneath the structure. This screen will gradually disappear in the structure when draft $\mathrm{T}_{1}$ is increased.

- $\quad$ The width is increased with flaps or doors at both sides of the structure. A certain amount of water cushions can be controlled by these doors. 


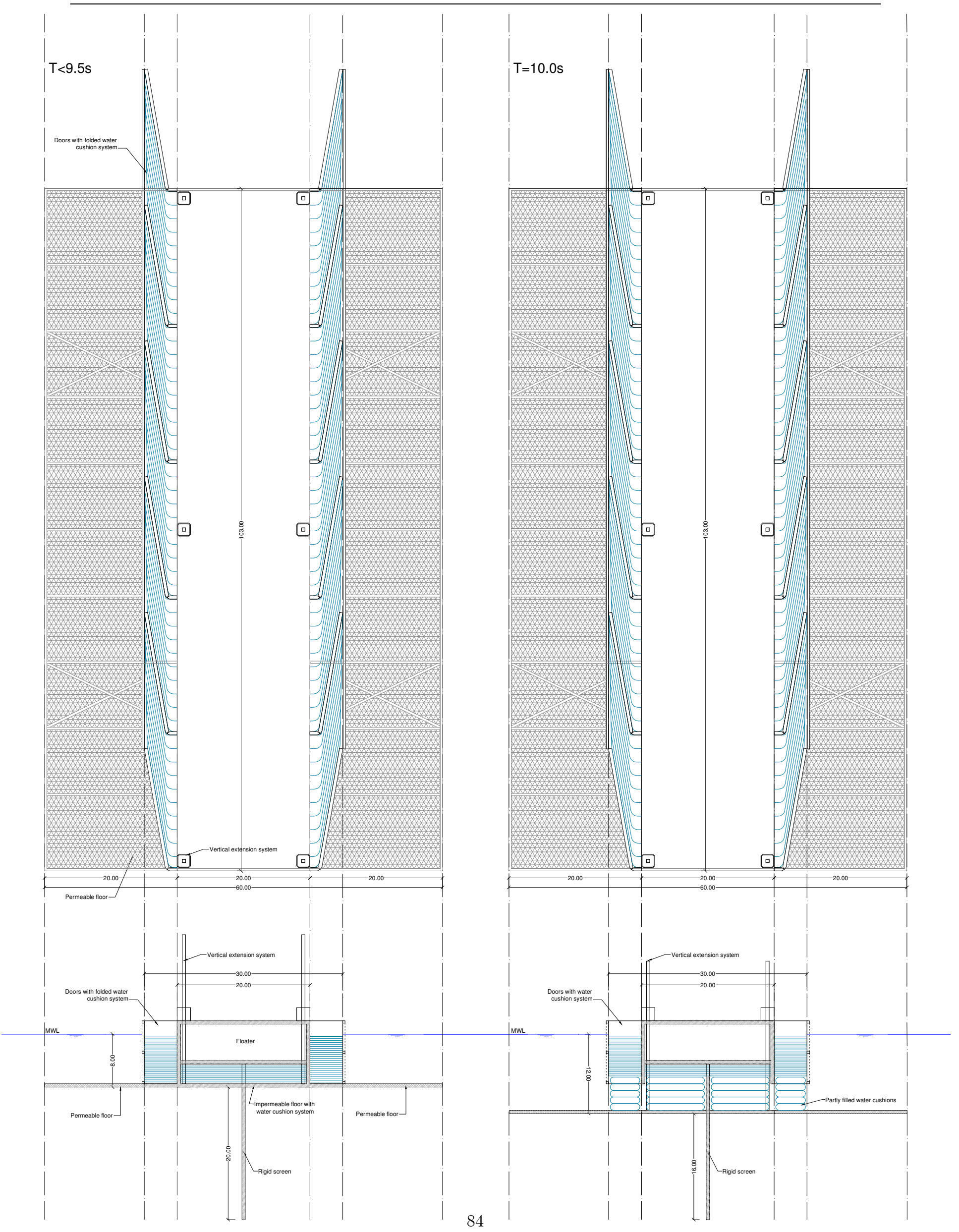




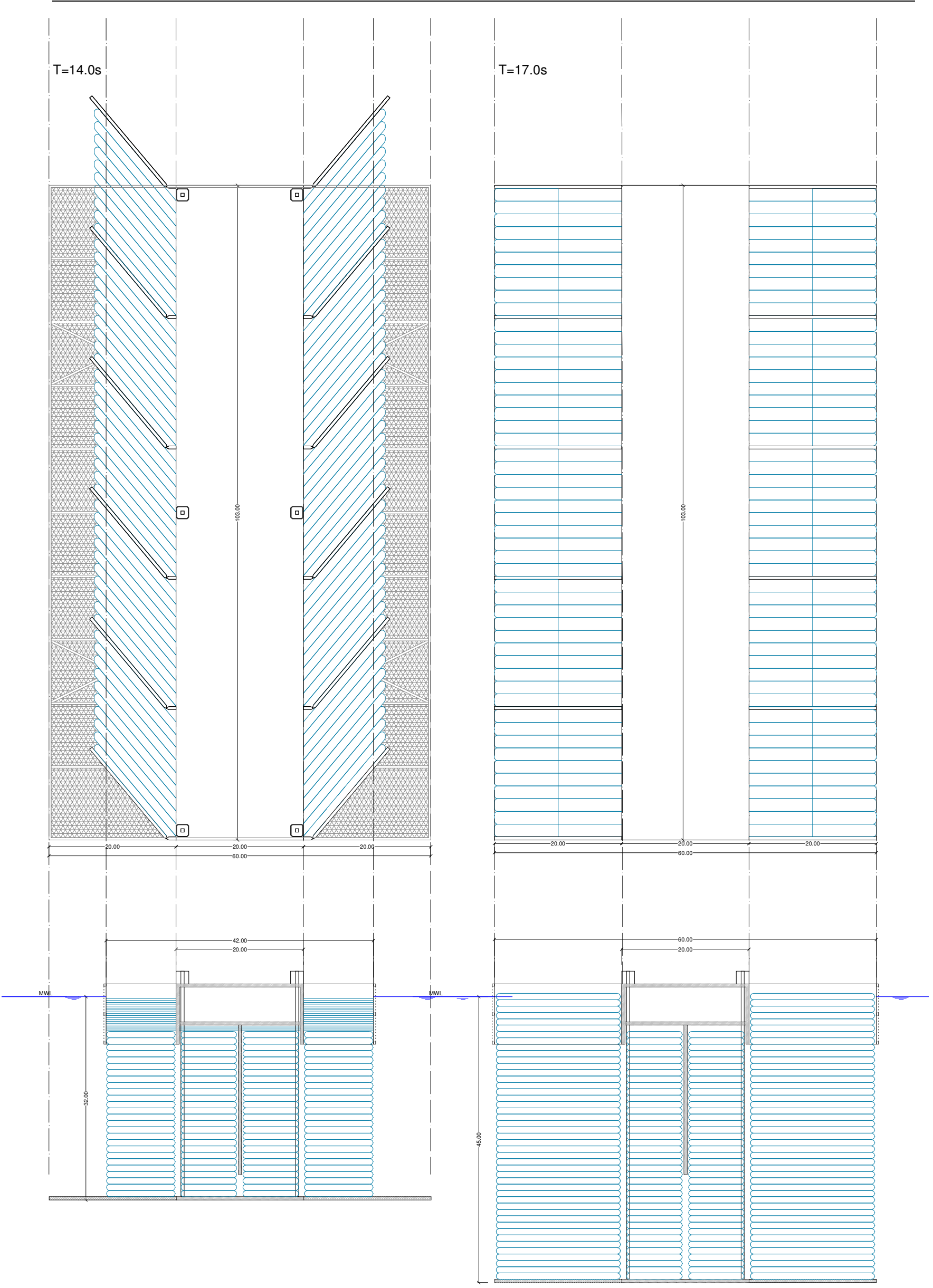


A three-dimensional artist impression of this structure is shown on the front cover of this report.

A couple of difficulties in design can be distinguished for this solution that will need extra attention:

The connection of the permeable bottom plate with the part of the water cushions that must be able to increase the width.

- $\quad$ The deformation of the water cushions when they are not guided by the flaps.

- $\quad$ The vertical draft increasing system. A solution may be found in the offshore technology, where vertical elevation systems are used to make stable working platforms. One of the examples is the wind turbine installation vessel 'the Mayflower' . This six-legged vessel can be jacked out of the water in order to create a stable working platform (fig 8-7).

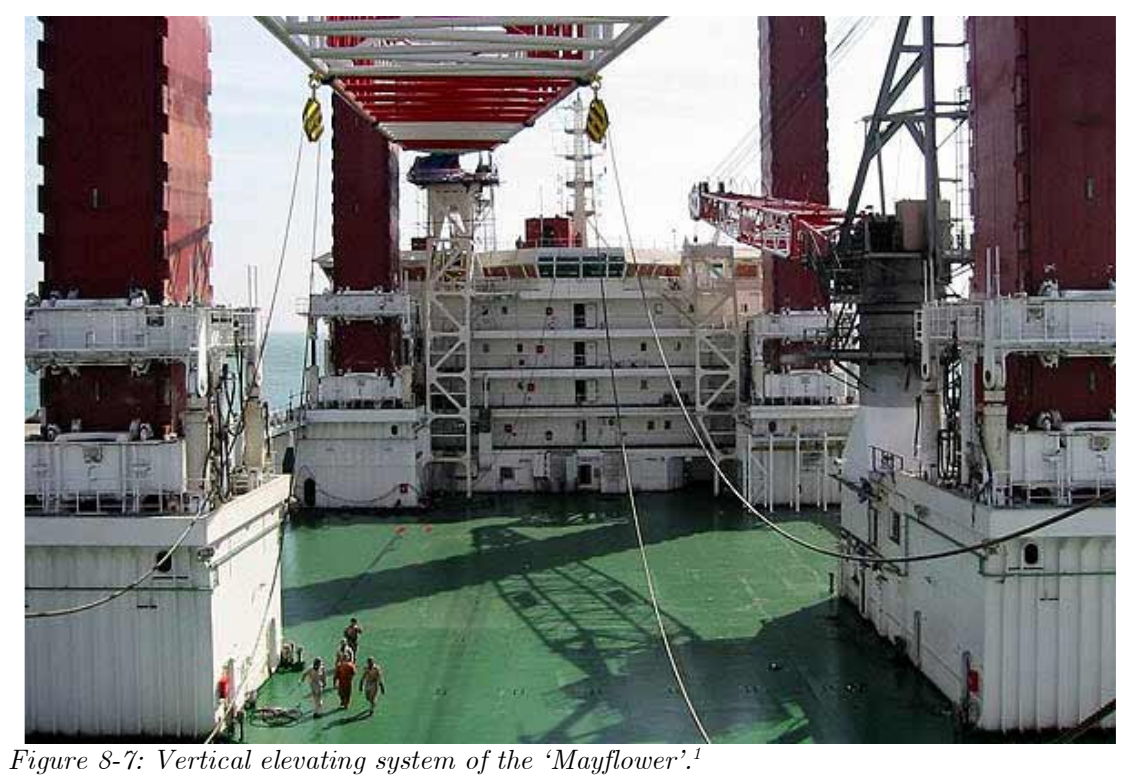

The material of the structure parts.

The filling and the folding of the water cushions.

The design as it is presented in appendix E could be one of the many possible structural solutions to the requirements of the floating breakwater. It is not said that this design is the solution since many structural elements will need much more attention. This example is only a suggestion of the writer on how to use the water as a material to increase the structural cross-section according to design graph 8-4.

In this primary stage of the study is it too early to say how the optimal floating breakwater design should look like. But it is important to realize that a floating breakwater of which the dimensions are determined by theoretical calculations must once be built in reality. The reality may therefore never be forgotten in the whole design process of the floating breakwater.

This chapter presented an example on how to connect the prevailing wave condition at a specific location (Durban in this case) with the calculation model as it was discussed in the previous chapters. In reality, the

\footnotetext{
${ }^{1}$ www.ship-technology.com
} 
local site conditions determine the performance as well as the design of the floating breakwater and the calculation model will only serve as a design tool. 


\section{Chapter 9}

\section{Conclusions and recommendations}

This chapter will summarize the most important conclusions that were stated during the course of this study. Although the contribution of several parameters to the performance of the floating breakwater was stated in this research, many questions raised that will need further research. Recommendations on the specification and the realisation of further research on this topic are resumed in this chapter.

A floating breakwater is an essential element for large-scale floating structures to become a success. In future applications, large-scale floating structures can be used as harbour terminals, such as Floating Transhipment Container Terminals. Previous studies have shown that the efficiency of such terminals can be improved significantly when wave attack is reduced by a floating breakwater system.

Lots of floating breakwater models were model-tested in the past. 'A floating breakwater is able to attenuate waves in a limited wave period domain' was the common conclusion in all these studies. This disappointing performance is the main reason why floating breakwaters have not been developed for commercial purposes.

The main objective of this thesis is:

Determine the influence of the hydrodynamic as well as the structural factors on the wave attenuating capacity of the floating breakwater and develop a calculation model that will serve as a design tool in developing the optimal floating breakwater.

In order to reach these objectives, a simplified calculation model is developed and calculation parameters are verified. The following assumptions are made in the calculation model:

- Only beam-on, regular waves are considered in order to determine the optimal floating breakwater lay-out for several wave periods.

- $\quad$ The influence of the mooring system as well as the connections between the floating breakwater sections on the hydrodynamic behaviour is neglected or user-defined.

- $\quad$ The part of the wave energy present underneath the floating breakwater is able to transmit for $100 \%$ to the lee ward side.

- $\quad$ The influence of wind is neglected.

- $\quad$ A full vertical dynamic wave pressure built-up is assumed to be possible underneath the floating breakwater, despite of the presence of a rigid screen underneath it.

- $\quad$ Energy losses in the zone around the floating body are neglected. 


\subsection{Conclusions}

- $\quad$ The performance of the floating breakwater is primarily determined by the draft-mass-width relation of the structure

- $\quad$ The width, draft, screen length and mass of a floating breakwater should be variable in order to reach optimal performance in a wide wave frequency range.

- $\quad$ It is theoretically possible to attenuate the waves transmitted by the underflow, sway and roll with the hydromechanical waves, generated by the heave motion when the phase shift of the heave motion is $-1 / 2 \pi$.

- $\quad$ An increase of the vertical mooring stiffness decreases the vertical motion and thus the wave attenuating capacity of the floating breakwater.

- $\quad$ Placing a rigid screen underneath the floating body of the floating breakwater has a positive contribution to the wave transmission when small period waves $(<10-13[\mathrm{~s}])$ are considered. For long period waves, such a screen will act as a sail and may even increase the wave transmission.

- A rectangular floating breakwater with a width of $18[\mathrm{~m}]$, a draft of $8[\mathrm{~m}]$ and a rigid screen of $18.5[\mathrm{~m}]$ is able to attenuate $95-100 \%$ of the wave amplitudes when wave periods smaller than 9 [s] are considered. For waves longer than 13 [s], a reduction of 65-70\% is obtained. An almost linear decay from $95-70 \%$ is visible when wave periods between 9 and 13 [s] are considered.

- $\quad$ A floating breakwater enables vessels to berth at all sides of the floating harbour until Beaufort scale 11. Without a floating breakwater system, only the leeward side of the floating harbour can be used as a berthing facility.

- $\quad$ The total system of floating breakwater and FTCT is able to create a safe haven for ships when weather conditions become bad.

- $\quad$ The optimal width/draft $\left(Y_{w} / T_{1}\right)$ ratio is around 3 when wave periods smaller than 5 [s] are considered and will decrease gradually when wave periods get larger. When this ratio is taken too small, the positive influence of heave on the wave transmission is diminished.

\subsection{Recommendations for further Research}

Many assumptions were used during the theoretical research, which are the basis for further research on this topic. Further research on the assumptions, but also improvements in design will be suggested in this section.

- Calibration of the calculation model is necessary to prove whether the magnitude of the transmission coefficients and the conclusions as they were discussed in the previous section need to be corrected. Model tests have to be performed in order to verify the results as they were presented in this report.

- $\quad$ The effect of oblique incoming waves, irregular waves, wave groups and bichromatic waves on the performance of the floating breakwater needs to be determined. The calculations and model tests 
in these wave conditions will provide valuable information on the performance of a floating breakwater in real ocean conditions.

- $\quad$ Although the three dimensional effects of diffraction was not involved in this research, it has influence on the three-dimensional wave transmission. The total floating breakwater-FTCT system has to be analyzed in order to determine the influence of all three-dimensional effects inside the harbour area.

- The measurements of Vugts $(1970)^{1}$ were used in this report to determine the magnitude of the hydrodynamic coefficients. Since Vugts performed his tests for models with width/draft ratios between 2 and 8, extrapolation was used to determine the hydrodynamic coefficients when this ratio was out of this domain. The effect of a screen on the hydrodynamic parameters was determined with DELFRAC. Model test should be performed to verify the assumptions as they were used in this study.

- The effect of the width on the underflow is not considered in this research. There may be a positive influence when the width is taken large enough. Jones $(1971)^{2}$ determined the positive influence of an increasing width relative to the wave length. Further, practical research is necessary to state the width-underflow relation in deep water conditions.

The effect of a single screen was determined theoretically in this report. Although it was proved that a rigid screen is an unnecessary structural addition when wave periods longer than 10-13 [s] are considered, the development of vortices may alter the results. The appliance of multiple, relative short screens, or screens with a certain permeability underneath the floating breakwater may result in an increase of the vortex development and thus a decrease of the transmitted wave energy.

The influence of the mooring system is assumed to be user-defined in the calculation model as it is used in this report. One can imagine that this may not be the case when real-time situations are considered. The development of a mooring line system with limited negative influence on the performance of the floating breakwater has to be investigated. Previous studies proved the fact that mooring forces get very big when dynamic response of the system gets very large or when strong currents are present at the site. This phenomenon may become a threat for further development of the floating breakwater when this subject is not studied very carefully.

The mutual connections between the floating breakwater elements are assumed not to have any effect on the hydrodynamic behaviour of the floating breakwater in this report. Development of connections that will not influence the heave motion is an important aspect for further research.

- Overtopping is not considered in this study. The effect of overtopping on the wave transmission and the hydrodynamic behaviour of the floating breakwater is a subject that has to be investigated.

The structural feasibility deserves more attention as it was presented in this report. A more detailed structural investigation has to be performed after the calculation model is checked by model tests.

\footnotetext{
${ }^{1}$ Vugts, J.H. (1970). PhD thesis 'The hydrodynamic forces and ship motions in waves', Delft University of Technology.

${ }^{2}$ Jones, D.B. (1971). 'Transportable breakwaters, a survey of concepts'. Technical Report R-727, Naval Civil Engineering Laboratory.
} 


\section{REFERENCES}

Ali, A. (2005). 'Floating Transhipment Container Terminal'. M.Sc thesis, Delft University of Technology, Delft, the Netherlands.

Briggs, M. , Ye, W., Demirbilek, Z. and Zhang,J. (2002). 'Field and numerical comparison of the RIBS floating breakwater'. Journal of Hydraulic Research vol.40, 2002, no.3.

D'Angremond, K. and Roode, F.C. van. (2001). 'Breakwaters and Closure dams'. Delft University Press, Delft, the Netherlands.

D'Angremond, K. and Tutuarima, W.H. (1998). Delft University of Technology, Delft, the Netherlands. Coastal Engineering 1998, Volume 2, Conference proceedings.

Battjes, Prof.dr.ir.J.A., 'Korte Golven', Eng: 'Short waves', Lecture notes, Delft University of Technology, Delft, the Netherlands.

BBR technologies. Picture on page 6. BBR news, special edition 2006.

Chiang C. Mei. (1983). 'The applied dynamics of Ocean surface waves'. Massachusetts Institute of Technology, Department of Civil Engineering.

European Container Terminals, ECT. Figure on page 9. www.ect.nl.

Evans, D.V. and Linton, C.M. (1991). 'Submerged floating breakwaters'. Journal of Offshore Mechanics and Arctic Engineering.

Faltinsen, O.M. (1995). 'Sea loads on ships and offshore structure'. Cambridge University Press.

Gardner, J.D., Townend, I.H. and Fleming, C.A. (1986). 'The design of a slotted vertical screen breakwater'. ASCE $20^{\text {th }}$ Coastal Engineering Conference.

Google Earth. October 2006.

Grotmaack, R and Meylan, M.H. 'Wave forcing of small floating bodies'. Institute of Information and Mathematical Sciences, Massey University. Auckland, New Zealand.

Heymann, E. (2006). 'Container shipping. Overcapacity inevitable despite increasing demand'. Deutsche Bank Research. Germany

Isaacson, M., Premasiri, S. and Yang, G. (1998). 'Wave Interactions with Vertical Slotted Barrier'. ASCE Journal of Waterway, Port, Coastal and Ocean Engineering.

Jones, D.B. (1971). 'Transportable breakwaters, a survey of concepts'. Technical report R-727, Naval Civil Engineering Laboratory, Port Hueneme, California, USA.

Journeé, J.M.J. and Massie, W.W. (2001). 'Offshore Hydromechanics, $1^{\text {st }}$ edition', Delft University of Technology, Delft, the Netherlands. 
Kee, S.T. and Kim, M.H. (1997). 'Flexible membrane wave barrier. II: Floating/submerged Bouy-membrane system'. Journal of Waterway, Port, Coastal and Ocean Engineering. ASCE

Koutandos, E.V., Karambas, Th.V. and Koutitas, C.G. (2004). 'Floating breakwater response to wave action using a Boussinesq model coupled with a 2DV Elliptic Solver'. Journal of Waterway, Port, Coastal and Ocean Engineering. ASCE

Kriebel, D.L. (1992). 'Vertical wave barriers. Wave transmission and wave forces'. ASCE $23^{\text {rd }}$ International Conference on Coastal Engineering.

Lenting, F.V. (2003). 'Een alternatief voor een verticale wand golfbreker'. Eng: 'An alternative for a vertical wall breakwater'. M.Sc thesis, Delft University of Technology, Delft, the Netherlands.

Murali, K. and Mani, J.S. (1997). 'Performance of cage floating breakwater'. Journal of Waterway, Port, Coastal and Ocean Engineering. July/August 1997.

New Civil Engineer (1994). 'Mulberry Harbour 1944-1994'. Supplement to New Civil Engineer, London, United Kingdom.

Newman, J.N. (1977). ‘Marine Hydrodynamics', MIT Press, Cambridge, United Kingdom.

Padmanabhan B. and Ertekin R.C. (2003). 'On the interaction of waves with Intake/discharge flows originating from a freely-floating body'. Journal of Offshore Mechanics and Arctic Engineering. ASME.

PIANC working group no.24 (1995). 'Criteria for movements of moored ships in harbours, a practical guide'.

PIANC working group no.13 (1994). 'Floating breakwaters, a practical guide for design and construction'.

Qui W., Peng H. and Chuang J.M. (2006). 'Computation of wave-body Interactions using the panel-free method and exact geometry'. Journal of Offshore Mechanics and Arctic Engineering. ASME.

Schepers, M.R. (1998). 'Kosten beschouwing van conventionele golfbrekers met betonelementen'. Eng: 'Cost evaluation of conventional breakwaters with concrete elements'. M.Sc thesis, Delft University of Technology, Delft, the Netherlands.

Seah, R.K.M. and Yeung, R.W. 'Sway and Roll hydrodynamics of cylindrical sections', University of California at Berkeley.

Silander, J. (1999). 'Floating Breakwaters and Environment', PhD thesis, Helsinki University of Technology, Helsinki, Finland.

Tirant, P.le. and Meunier, J. (1990). 'Anchoring of floating structures'. Association de Recherche en Géotechnique Marine (ARGEMA).

Tolba, E.R.A.S. (1998). 'Behaviour of floating breakwaters under wave action' PhD thesis, Suez Canal University of Egypt.

Tsinker, G. (1995). Marine Structures Engineering: Specialized Applications, Chapman and Hall, New York, USA. 
Vugts, J.H. (1968). 'The hydrodynamic coefficients for swaying, heaving and rolling cylinders in a free surface'. Report no.112 S, Netherlands ship research centre TNO. Delft, the Netherlands.

Vugts, J.H. (1970). 'The hydrodynamic forces and ship motions in waves', Delft University of Technology, Delft, the Netherlands.

Wijnolst, N. , Scholtens, M. and Waals, F. (1999). 'Malacca-max: the ultimate container carrier'. Delft University Press.

Williams, A.N. and McDougal, W.G. (1996). 'A dynamic submerged Breakwater'. Journal of Waterway, Port, Coastal and Ocean Engineering. ASCE

Yamamoto, T. and Yoshida, A. (1979). 'Large wave tank tests on taut-moored breakwaters'. Coastal structures '79, Vol.II, ASCE. 


\section{LIST OF APPENDICES}

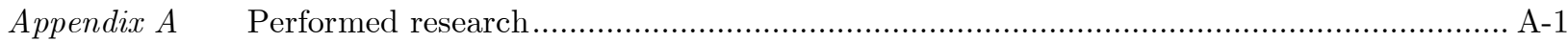

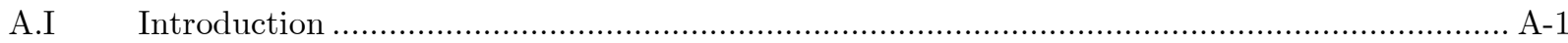

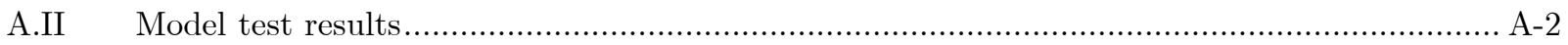

A.II.a Influence of the degree of freedom ................................................................................ A-2

A.II.b Influence of the structural dimensions ............................................................................ A-6

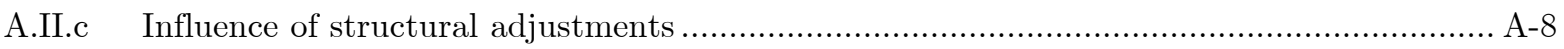

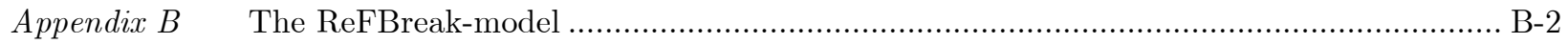

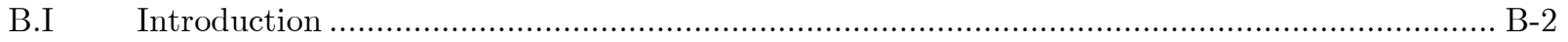

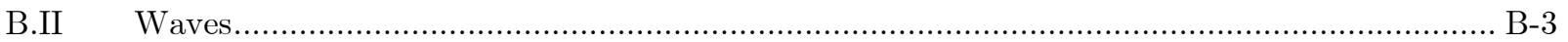

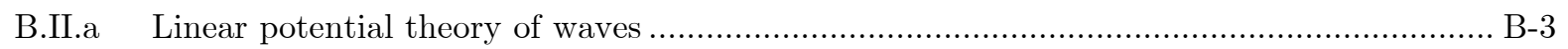

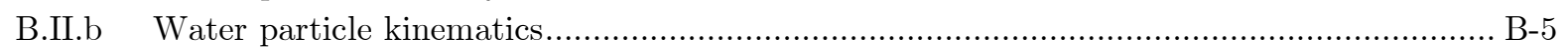

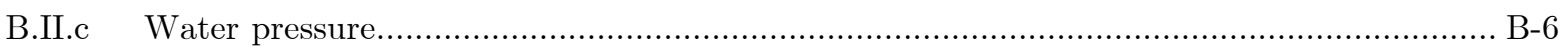

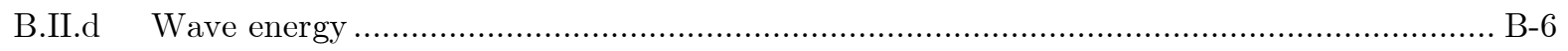

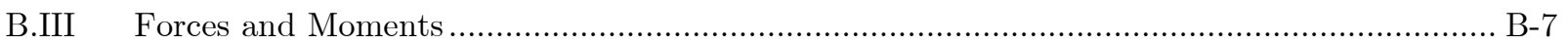

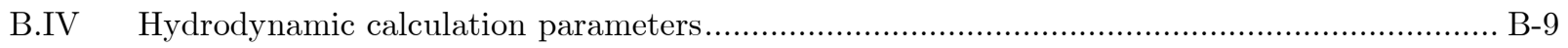

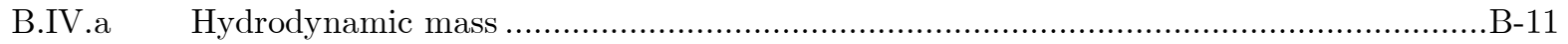

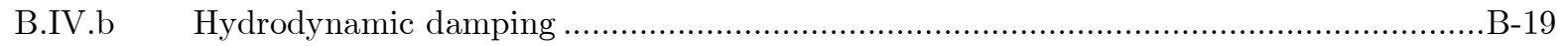

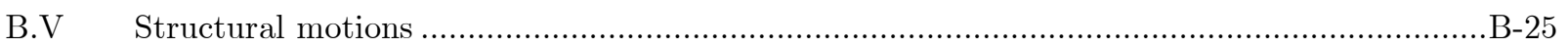

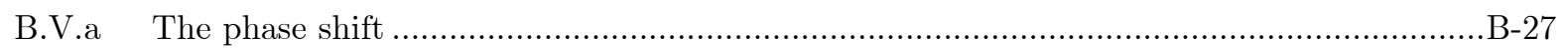

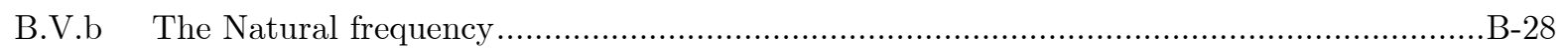

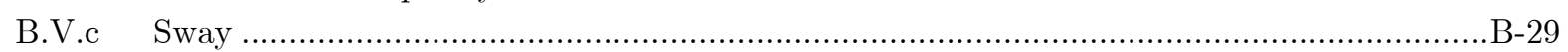

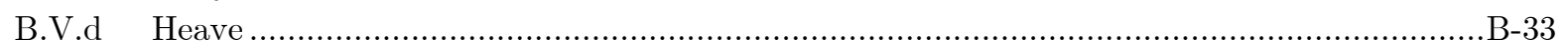

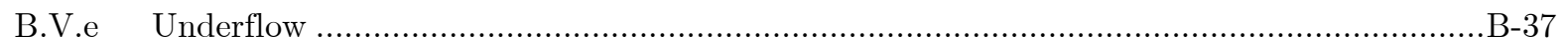

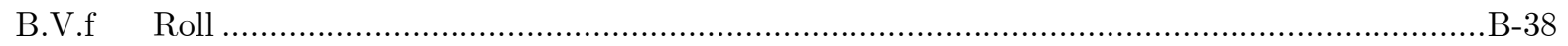

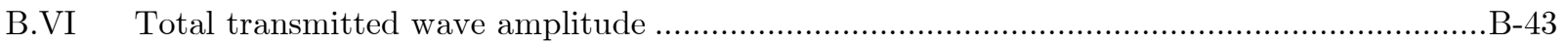

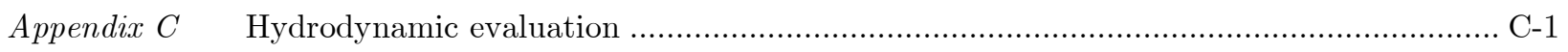

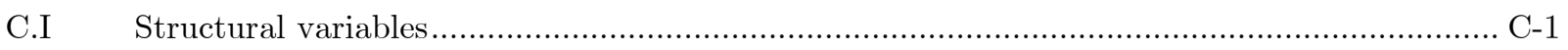

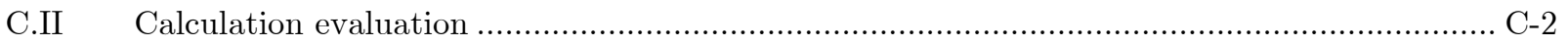

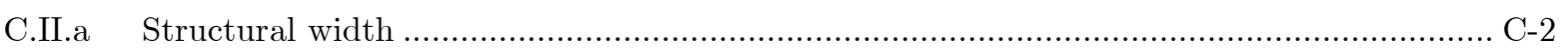

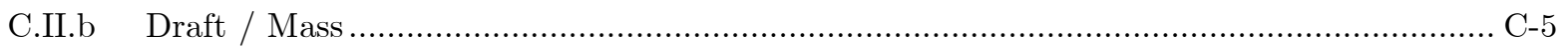

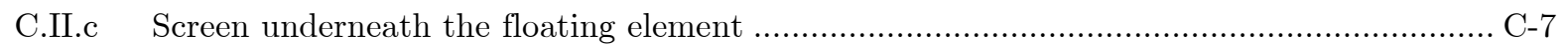

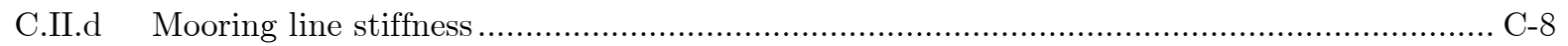

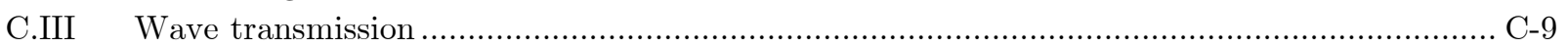

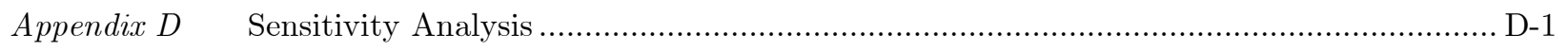

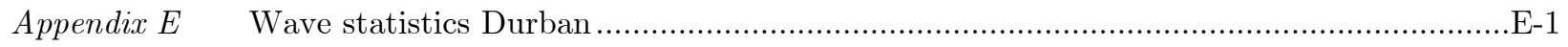




\section{Appendix A}

\section{Performed research}

\section{A.I Introduction}

In general, one can distinguish two types of floating breakwaters, based on their hydraulic system behaviour. These are the reflective systems and the dissipative systems or combinations of both. Within these two categories, huge amounts of floating breakwater designs have been created by creative engineers. Examples of these are listed below:

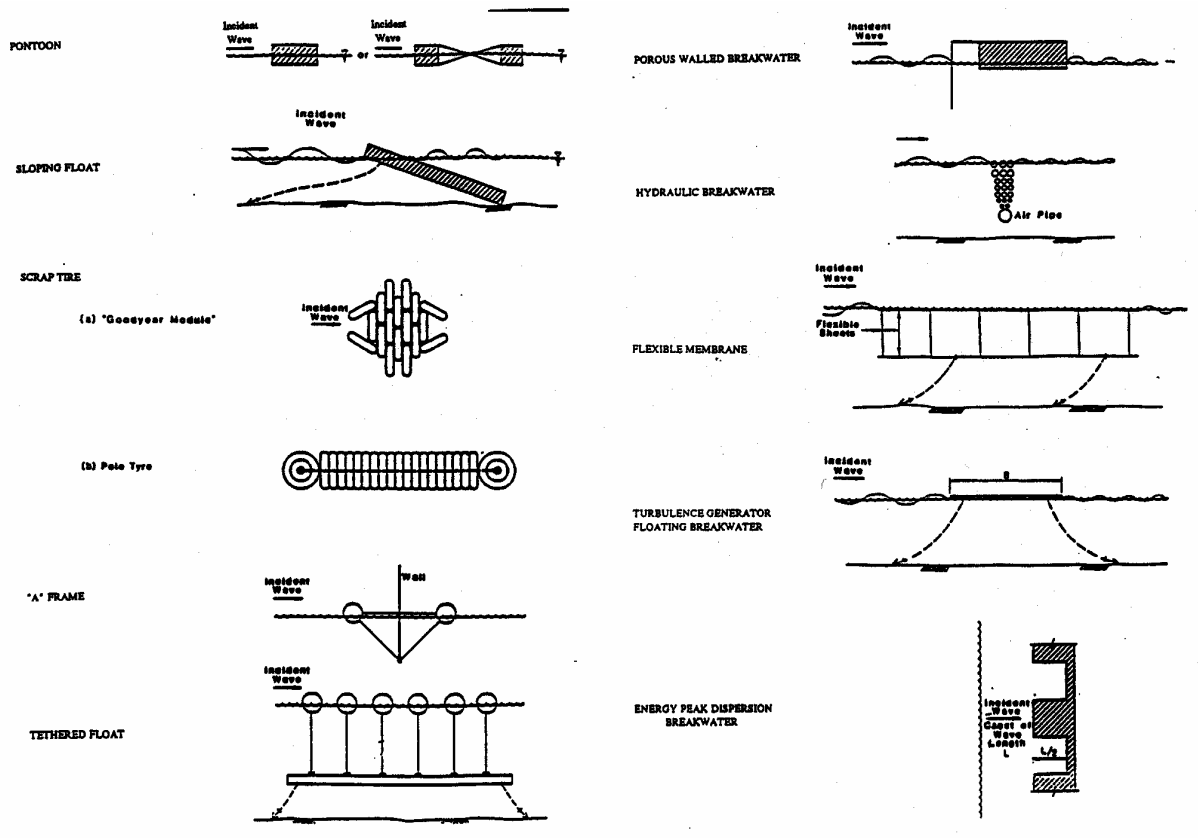

Figure A-1: General floating breakwater configurations. ${ }^{1}$

Although the tested models show an enormous variation in design (and performance), the main common research topics can be divided into 6 areas which are more or less related to one another by means of their influencing factors. These 6 areas and their influencing factors are shown in figure A-2.

The effect of the several parameters on the performance of the system can be either positive or negative, depending on the wave situation and the structural execution. The common problem of all the designed floating breakwaters has always been the bad or limited performance. During this section, an overview is given of some of the performed model tests and the conclusions that were gained from these tests.

Figure A-2: Research areas into the dynamic behaviour of floating breakwaters.

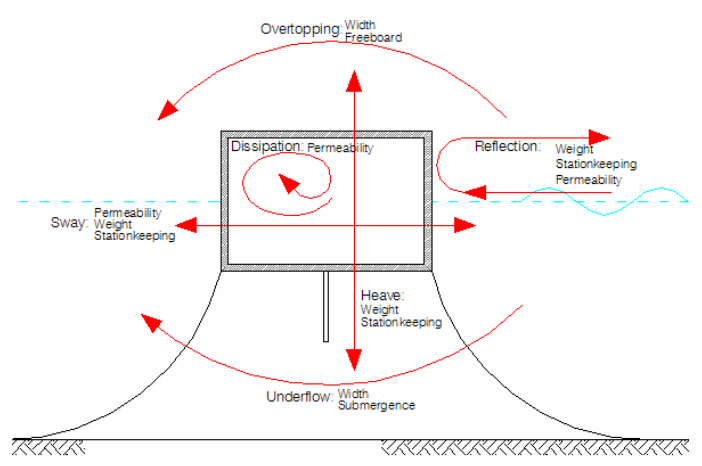

${ }^{1}$ PIANC report of working group no. 13 (1994). 'Floating breakwaters. A practical guide for design and construction'. 


\section{A.II Model test results}

A huge amount of model tests were performed in the past. In these tests, the model design as well as the scope of the tests showed a lot of variation. Despite this variation, the conclusions of all of the tests can be summarized into three categories with their influence factors:

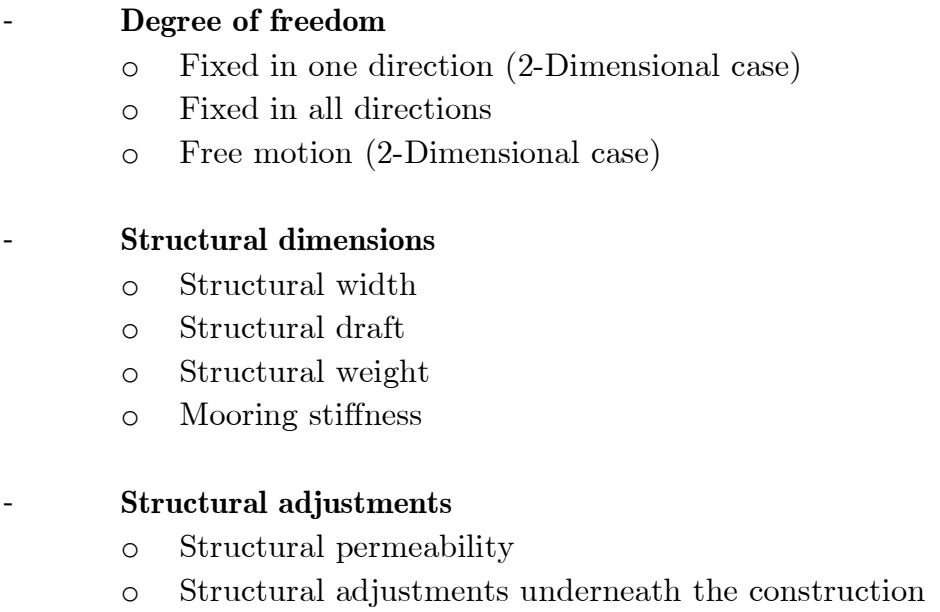

All the model tests were performed in a wave flume. Although no deep-water waves were created in these flumes, the tests will give a certain insight in the influence of the various factors on the attenuating capacity of the floating breakwater.

\section{A.II.a Influence of the degree of freedom}

\section{Heave}

Test 1: Tolba ${ }^{1}$

[Tolba] investigated the behaviour of floating breakwaters under wave attack and the influence of the vertical motion in particular. In his report, the concentration is on a floating breakwater with a vertical pile-anchorage. The floating breakwater is able to move in a vertical direction, while the horizontal motions are diminished by the piles. Although the tests are performed in varying (transitional / deep) water conditions, the influence of the heave motion on the performance of the floating breakwater is clear. Figures A-3 and A-4 show the values of $\mathrm{C}_{\mathrm{r}}$ and $\mathrm{C}_{\mathrm{t}}$ for both restrained and the heave motion floating breakwater, plotted versus $\mathrm{B} / \mathrm{L}$ and $\mathrm{d} / \mathrm{L}$ for different values of $\mathrm{D} / \mathrm{d}$.

Where:

$\begin{array}{llll}- & \mathrm{D}: & \text { Draft of the structure } \\ - & \mathrm{d}: & \text { Water depth } \\ - & \mathrm{B}: & \text { Width of the structure } \\ - & \mathrm{L}: & \text { Wave length } \\ - & \mathrm{C}_{\mathrm{t}}: & \text { Transmission coefficient } \\ - & \mathrm{C}_{\mathrm{r}}: & \text { Reflection coefficient }\end{array}$

[Tolba] concludes:

The figures show that the values of $\mathrm{C}_{\mathrm{r}}$ and $\mathrm{C}_{\mathrm{t}}$ the floating breakwater moving only in heave motion and fixed in position using vertical piles are always lower than the results for the restrained structure for all the values of $\mathrm{D} / \mathrm{d}$. The explanation of these results is that in the case of the restrained structure the total energy of the incident wave will be divided into three parts, the energy of the transmitted wave, the energy of the reflected wave and the energy lost, while in the case of the floating breakwater there is an additional source of lost energy, which is the energy lost in inducing the heave motion of the body.

${ }^{1}$ Tolba, E.R.A.S. (1998). 'Behaviour of floating breakwaters under wave action', PhD thesis, Suez Canal University of Egypt 
The experiments, executed by Tolba, prove the positive effects of the heave motion on the wave attenuating capacity of the floating breakwater. It is also clear that the performance of the floating breakwater is dependent on the dimensions of the construction. This will be clarified in section A.II.b.

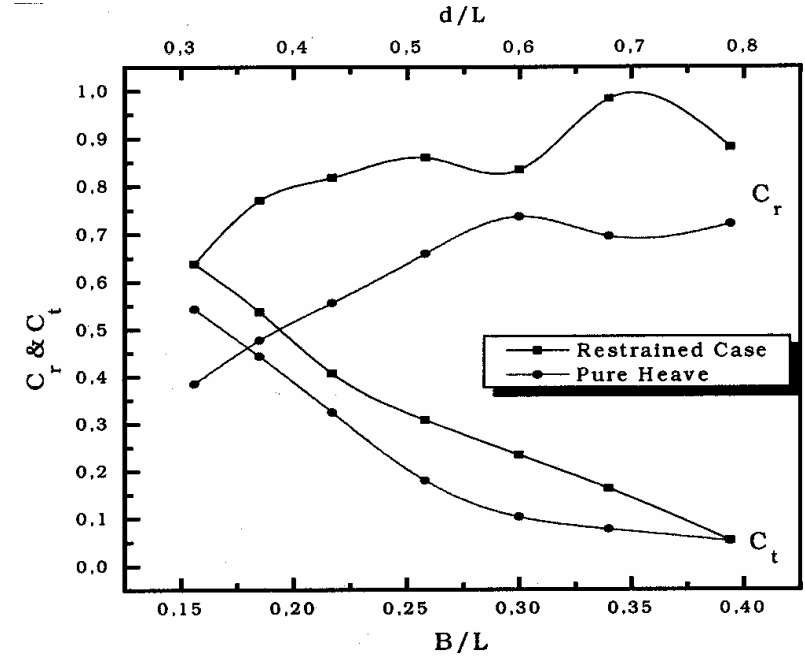

Figure A-3:Effect of the heave motion on $C_{r}$ and $C_{t}$ $\left(D / d=1 / 5, B / d=1 / 2, H_{i} / L=0.0485\right)$

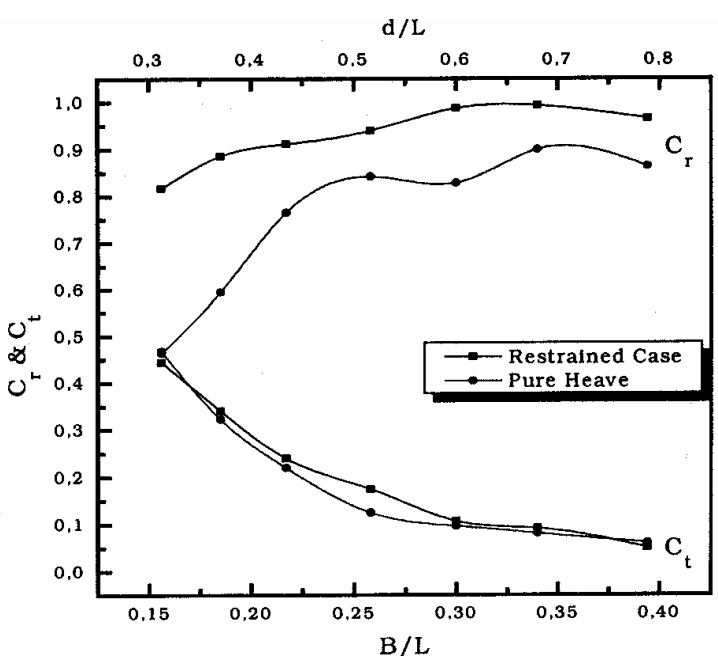

Figure A-4:Effect of the heave motion on $C_{r}$ and $C_{t}$ $\left(D / d=1 / 4, B / d=1 / 2, H_{i} / L=0.0485\right)$

Test 2: Koutandos et al. ${ }^{1}$

In their research, [Koutandos et al.] $]^{1}$ studied the hydrodynamic behaviour of fixed and heave motion floating breakwaters, using a finite difference, mathematical model based on the Boussinesq type equations.

A simple, two-dimensional rectangular floating body is considered. Since the final goal of this research is to study floating breakwaters efficiently in shallow and intermediate waters, the research does not apply to deep water situations as they are considered in this report. The authors use the Laplace equation to state the incompressibility of the fluid. A confined flow arises underneath the construction, which is a divergent situation in comparison to the deep water situation. However, understanding the influence of heave on the performance of the floating breakwater is gained in this research.

The calculation results are compared with the experimental results of [Tolba $]^{2}$ and the analytical results of $[\text { Drimer et al. }]^{3}$.

The graphs, as they are reflected in figures A-5 and A-6, show a good similarity in comparison with the experimental results of $[\text { Tolba }]^{2}$. In the figure, the following symbols are used:

$\begin{array}{llll}- & \mathrm{dr}: & \text { Draft of the structure } \\ - & \mathrm{h}: & \text { Water depth } \\ - & \mathrm{B}: & \text { Width of the structure }\end{array}$

Figures A-5 and A-6 show different results due to the changed draft of the structure. Striking is the fact that the heave motion model performs better or as good as the restrained model. An increase of the draft leads to better results as well.

[Koutandos et al.] explains this phenomena as follows:

By comparing figures A-5 and A-6, we can see the effect of the heave motion in the performance of the floating breakwater. In both cases the breakwater presents the same trends in the specific graphs with a slightly better performance in the heave motion model case. The performance of the heave motion floating breakwater is directly linked to the phase difference between the partially standing wave formed in the front part of the structure and the motion of the heave motion breakwater. When the radiated waves cancel the transmitted waves the heave motion breakwater performs better than the restrained one.

\footnotetext{
${ }^{1}$ Koutandos,E.V., Karambas, Th.V. and Koutitas, C.G. (2004). 'Floating Breakwater Response to Waves Action Using a Boussinesq Model Coupled with a 2DV Elliptic solver', ASCE Journal of waterway, port, coastal and ocean engineering.

${ }^{2}$ Tolba, E.R.A.S. (1998). 'Behaviour of floating breakwaters under wave action', PhD thesis, Suez Canal University of Egypt

${ }^{3}$ Drimer, N., Agnon, Y., and Stiassnie, M. (1992). 'A simplified analytical model for a floating breakwater in water of finite depth'
} 
This conclusion corresponds to the conclusion done by [Tolba]. Heave has a positive influence on the wave attenuating capacity of the floating breakwater.

\begin{tabular}{|lll|}
\hline Explanation of graphs: & & Drimer et.al. (1992) \\
& & Experiments Tolba (restrained) (1998) \\
& Present model (restrained) \\
& Experiments Tolba (heave motion) (1998) \\
\hline$-\ldots-\cdots$ & Present model (heave motion) \\
\hline
\end{tabular}
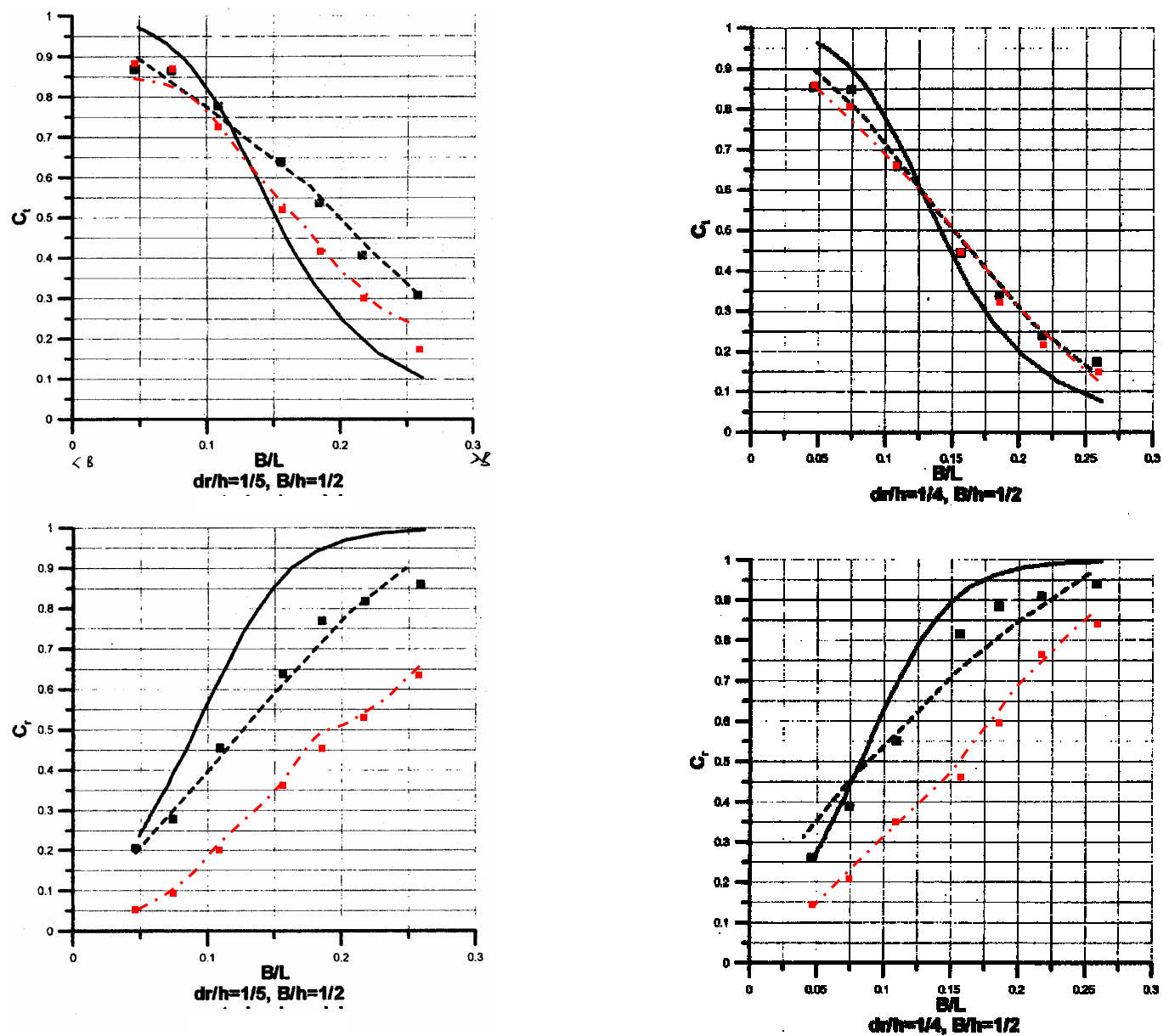

Figure A-5: Variation of $C_{t}$ and $C_{r}$ against B/L. $(d r / h=1 / 5, B / h=1 / 2) \quad$ Figure A-6: Variation of $C_{t}$ and $C_{r}$ against B/L. $(d r / h=1 / 4$, $B / h=1 / 2)$ 


\section{Sway}

Preventing sway is the main reason why [Tolba] used vertical poles as anchorage for his floating breakwater. He wanted the positive effects of the heave motion and not the negative influence of the (horizontal) sway motion.

Test 3: Silander ${ }^{1}$

In his PhD thesis, [Silander] proved that the assessment of [Tolba] is indeed correct.

In his research, [Silander] used a huge amount of breakwater shapes (35), each with an own width, draft, mooring stiffness, weight and front or rear barrier permeability. This was done in order to state the contribution of each of these factors to the wave attenuating capacity of the floating breakwater.

It can be concluded from figure A-7 that the sway motion has a negative contribution to the wave transmission. An increase of the mooring stiffness reduces the negative contribution of the sway motion.

The following symbols are used in this figure:

$$
\text { - } \quad \mathrm{K}_{\mathrm{t}}: \quad \text { Transmission coefficient }
$$

The construction codes mean: as an example: W10R30 or W10S30

$$
\begin{array}{llll}
- & \text { W }: & \text { Width of the structure }=0.40 \mathrm{~m} \\
- & 10: & \text { No screen underneath the structure } \\
- & \mathrm{R}: & \text { Fixed mooring } \\
- & \mathrm{S}: & \text { Swaying motion possible } \\
- & 30: & \text { Type of anchorage, depending on the stiffness }
\end{array}
$$

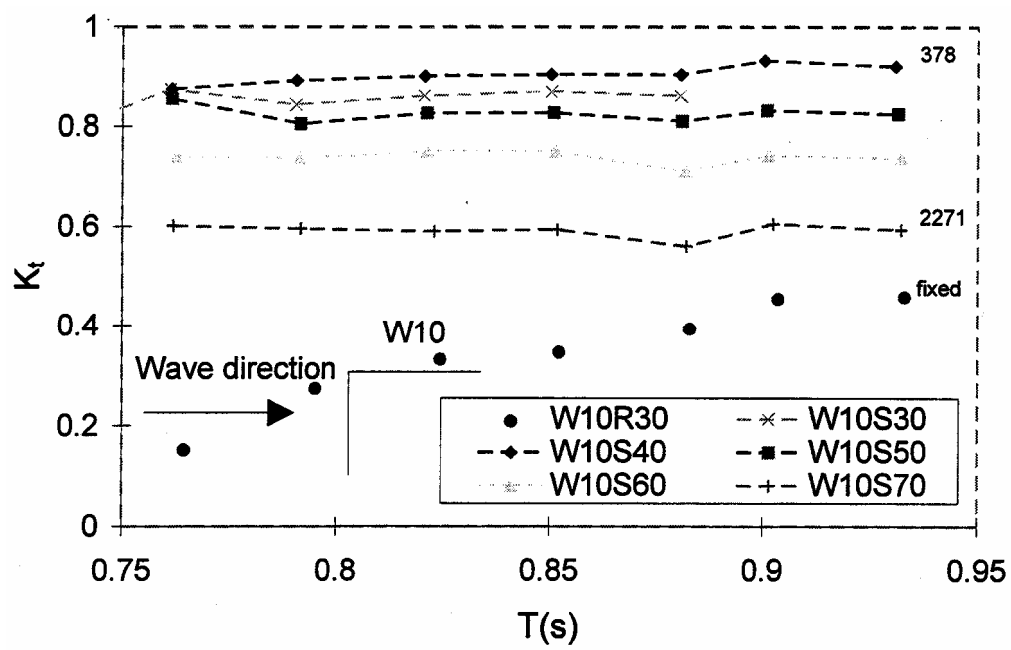

Figure A-7: Transmission through single swaying barrier ( ${ }^{* *} S$,---) in the wave flume. (W10S**) stands for different spring systems. Structures had the same draft and no pipes as indicated in figure. The spring constant was $378 \mathrm{~N} / \mathrm{m}$ for W10S40 and 2271 $N / m$ for W10S70.

\footnotetext{
${ }^{1}$ Silander, J., (1999), 'Floating breakwater and environment', Helsinki University of Technology.
} 


\section{A.II.b Influence of the structural dimensions}

\section{Structural width \& degree of freedom}

Test 4: Silander ${ }^{1}$

Structural dimensions do influence the wave transmission, as [Silander] proved in his PhD thesis. In figure A-8, 5 structure types are compared. From this figure it can be concluded that normally anchored (free-motion) structures can perform better at certain wave periods than a fixed structure does. This is the case for the wide structure in the period rang between 0.87 and 1.02 seconds. The narrow free structure does not perform well in this test. This may be due to the fact that a narrow structure is not able to generate (positive) heave waves as high as a wide structure does.

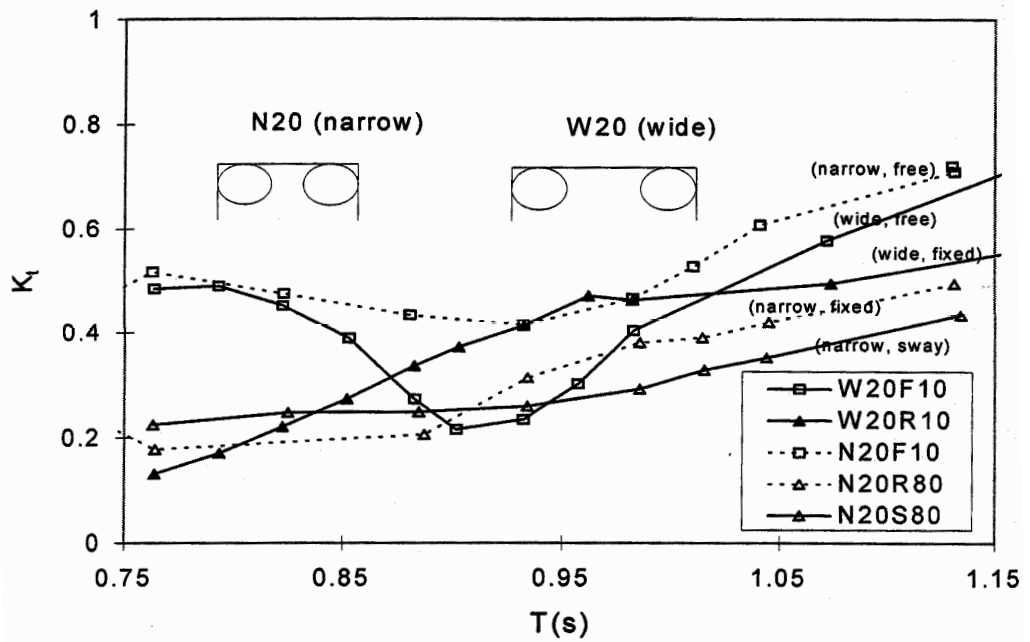

Figure A-8: Transmission through normally anchored 0.40m wide (W20F) and 0.30m wide (N20F) structures having the same draft. The $\left({ }^{* *} S\right)$ for sway motion and $\left({ }^{* *} R\right)$ is for fixed structure.

The narrow, sway motion structure (N20S80) produces relative low transmission coefficients over a large period range. This narrow sway motion structure performs almost the same as the same structure in a fixed mode (N20R80) does. Although model N20S80 is able to move horizontally, this motion is restricted by a relative stiff horizontal mooring. The relation that indicates when a sway-motion structure can be assumed to be fixed is estimated by [Silander] as: horizontal motion $/ \mathrm{H}_{\mathrm{i}}<0.30$.

\footnotetext{
${ }^{1}$ Silander, J., (1999), 'Floating breakwater and environment', Helsinki University of Technology.
} 


\section{Structural draft}

Test 5: Tolba ${ }^{1}$

In test 2 [Koutandos et al. $]^{2}$ already proved the positive effect of an increasing draft on the wave transmission.

In his research, [Tolba] proved the positive effect of draft on the wave transmission coefficient. A cheap solution to realize this is to put a vertical plate underneath the floating body. Test results (figure A-9) prove that this is indeed a valuable addition to the construction.

In his conclusion, [Tolba] writes:

An attempt was made to increase the efficiency of the suggested floating breakwater which moves only in heave motion. The investigation showed that the efficiency of the floating breakwater can be increased by adding a vertical rigid plate under the floating body. This will cut the costs of construction of the rectangular cross sectional area required to produce the same wave attenuation.

However the effects of a permeable screen or more than one screens were not included in this research.

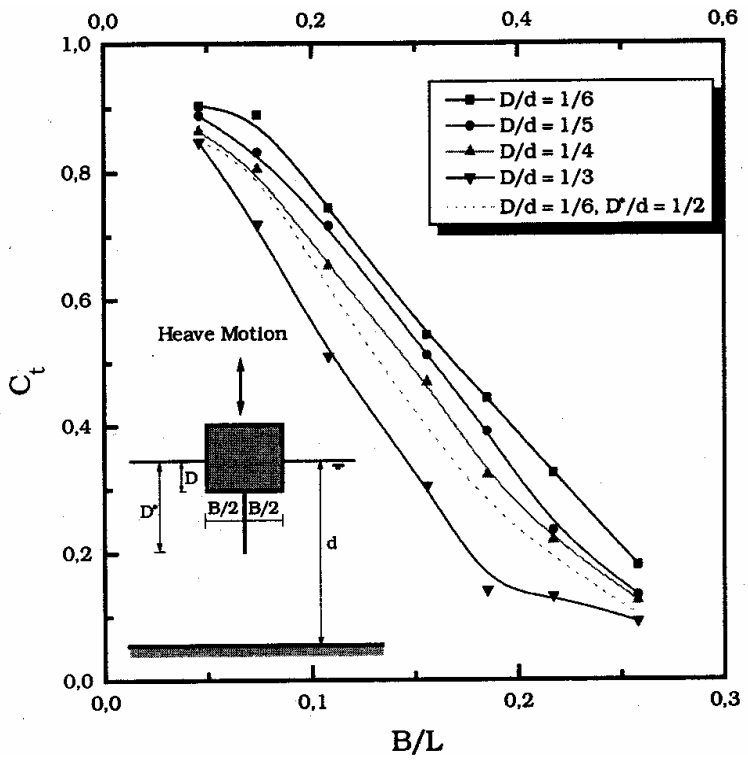

Figure A-9: Improving of floating breakwater efficiency using a vertical plate.

\section{Structural weight}

Although the weight of the structure is an important parameter that determines the hydrodynamic behaviour of the structure, no special attention is paid to this subject.

\footnotetext{
${ }^{1}$ Tolba, E.R.A.S. (1998). 'Behaviour of floating breakwaters under wave action', PhD thesis, Suez Canal University of Egypt

${ }^{2}$ Koutandos,E.V., Karambas, Th.V. and Koutitas, C.G. (2004). 'Floating Breakwater Response to Waves Action Using a Boussinesq Model Coupled with a 2DV Elliptic solver', ASCE Journal of waterway, port, coastal and ocean engineering.
} 


\section{A.II.c Influence of structural adjustments}

\section{Structural permeability}

A permeable structure creates turbulence in the water which leads to a wave energy reduction. On the other hand, a reduced sway motion, and thus a lower transmitted wave height due to this motion is obtained.

Test 6: Silander ${ }^{1}$

[Tolba] investigated the influence of a varying draft and the influence of a rigid plate underneath the construction. He did not include the influence of structural permeability on the performance of the floating breakwater. To state these permeability effects, [Silander] did tests with constructions of varying permeability. Besides this, he also involved the possibility of having two screens with different permeability's underneath the construction.

Figure A-10 shows the influence of permeability for sway motion and fixed structures. It is clear from this figure that permeability has a huge (negative) influence on the performance of a fixed floating breakwater.

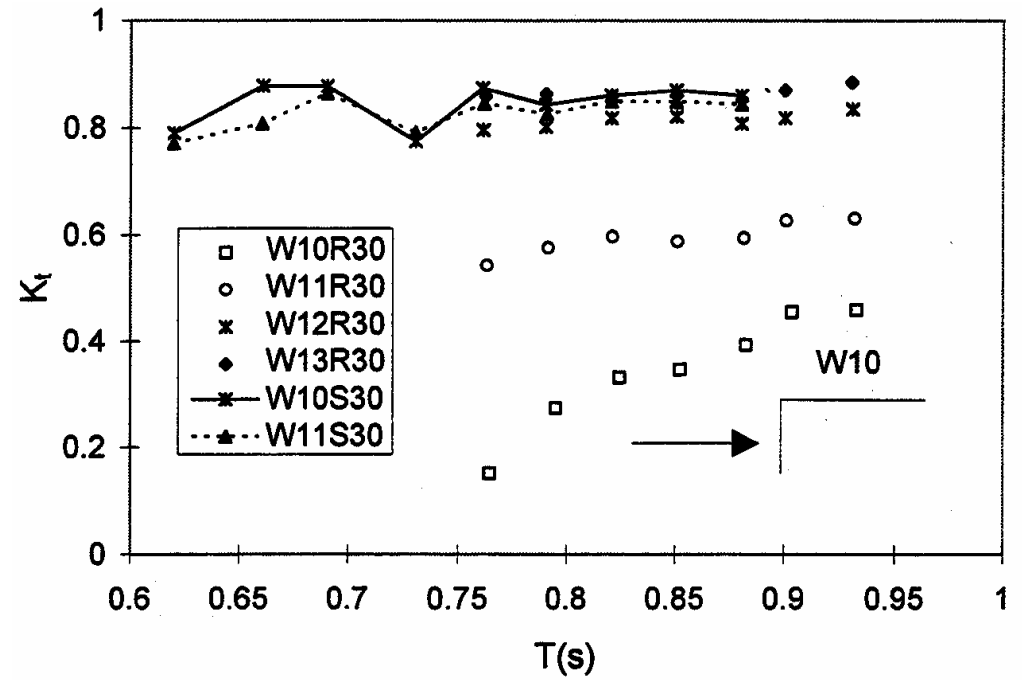

Figure A-10: Transmission coefficient for a fixed $\left({ }^{* * *} R\right)$ and a swaying $\left({ }^{* *}\right.$ S) single barrier without pipes (same draft). Four different permeability's (0, 0.19, 0.32 and 0.41) corresponding to (W10, W11, W12 and W13).

In his report, [Silander] describes the efficiency of a floating breakwater as the amount of (anchoring) force required to provide a certain attenuating performance. Since the amount of anchoring force is directly related to the wave reflection of the system, this seems to be a logical description.

${ }^{1}$ Silander, J., (1999), 'Floating breakwater and environment', Helsinki University of Technology. 
A two barrier system shows a huge improvement on the performance of the floating breakwater. Figure A-11 shows this effect for several constructional executions. In his report, [Silander] proves that the wave transmission will mainly depend on the wave length, the space between the barriers and the draft.

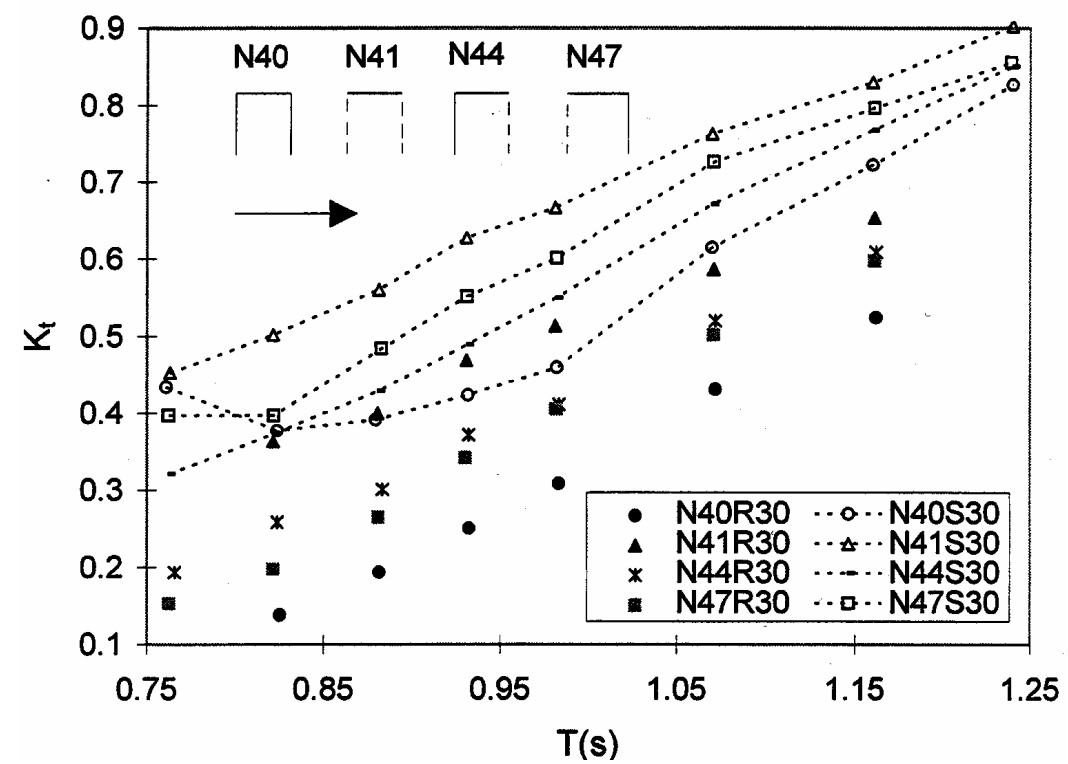

Figure A-11: Transmission through fixed ( ${ }^{* * *} R$, dots) and swaying ( ${ }^{* * *}$ S, ---) structures (no pipes). Permeability was 0 or 0.19 for front barrier or back barrier. For example $N 44$ has a solid barrier on seaside 'front' and a permeable on leeward side 'back' (0.19 permeability).

The effect of permeability on free floating structures is shown in figure A-12

It can be concluded that the structure with solid barriers (N20) will attenuate waves better when the wave period is higher than $0.92 \mathrm{~s}$. The permeable barrier at the front (N24) is shown to be better attenuating waves than (N20) when the wave period is less than $0.92 \mathrm{~s}$.

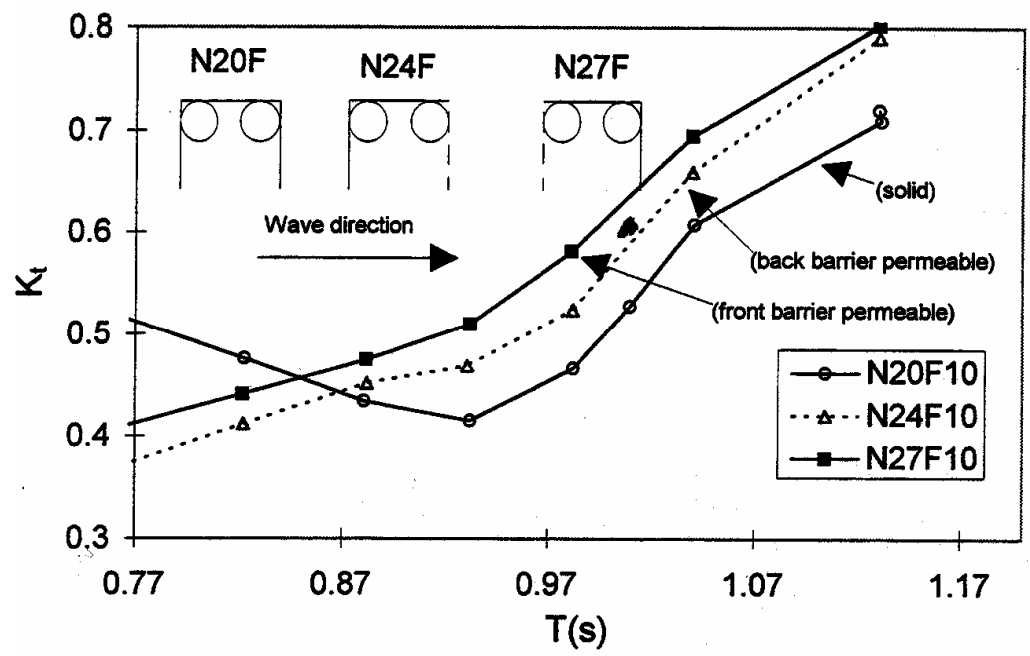

Figure A-12: Transmission through free floating structures. Permeability 0 and 0.19 was used.

In their research, [Murali et al. $]^{1}$ proved that it is possible to gain a 15-20\% reduction of the transmitted wave height when a screen of vertical pipes, separated by a gap, is installed.

${ }^{1}$ Murali, K. and Mani, J.S. (1997). 'Performance of cage floating breakwater'. ASCE Journal of waterway, port, coastal and ocean engineering. 


\section{Appendix B}

\section{The ReFBreak-model}

\section{B.I Introduction}

In this model, a rectangular two-dimensional floating body will be considered. The floating body is able to move in the three degrees of freedom:

$\begin{array}{llll}\text { Horizontal } & : & \text { Sway } & (2) \\ \text { Vertical } & : & \text { Heave } & (3) \\ \text { Rotational } & : & \text { Roll } & (4)\end{array}$

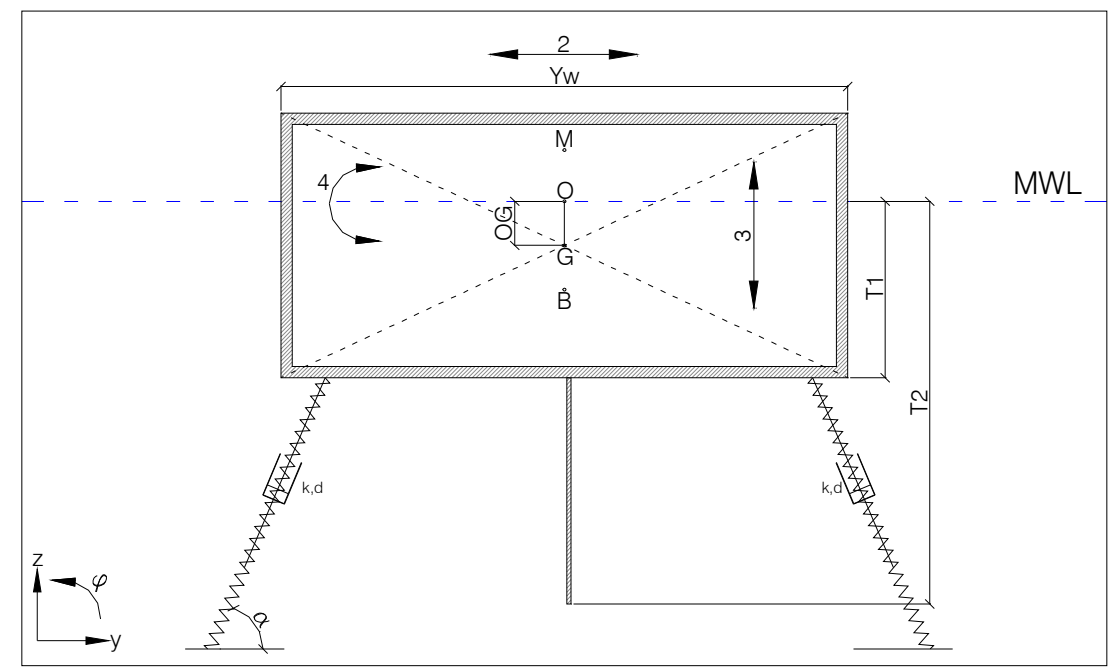

Figure B-1: Two-dimensional model lay-out.

The parameters that will be considered are shown in figure B-1. In chapters B.III and B.IV calculations are performed in order to state the hydrodynamic calculation parameters as well as the hydrodynamic behaviour of constructions with different rectangular cross sections. The considered structures are listed in the table below:

\begin{tabular}{|c|c|c|c|c|c|c|c|c|c|c|c|}
\hline \multirow{3}{*}{$\begin{array}{l}\text { Structure } \\
\text { number. }\end{array}$} & Length & Width & $\begin{array}{c}\text { Floater } \\
\text { draft }\end{array}$ & $\begin{array}{l}\text { Width/ } \\
\text { draft }\end{array}$ & $\begin{array}{c}\text { Screen } \\
\text { draft }\end{array}$ & Mass & $\begin{array}{l}\text { Mass moment } \\
\text { of inertia }\end{array}$ & $\begin{array}{l}\text { Centre of gravity } \\
\text { below waterline }\end{array}$ & $\begin{array}{l}\text { Mooring } \\
\text { stiffness }\end{array}$ & $\begin{array}{l}\text { Mooring } \\
\text { damping }\end{array}$ & $\begin{array}{c}\text { Mooring } \\
\text { angle }\end{array}$ \\
\hline & $L_{w}$ & $Y_{w}$ & $T_{1}$ & $Y_{w} / T_{1}$ & $T_{2}$ & $\mathrm{~m}$ & $\mathrm{I}_{\mathrm{xx}}$ & $\overline{O G}$ & $k$ & $d$ & $\alpha$ \\
\hline & $m$ & $m$ & $m$ & - & $m$ & ton & t. $m^{2}$ & $m$ & $\mathrm{~kg} / \mathrm{m}^{2}$ & $\mathrm{~kg} / \mathrm{m}$ & deg. \\
\hline 1 & 500 & 25 & 7.80 & 3.2 & 7.80 & 99,938 & $7.65 \cdot 10^{6}$ & 1.95 & - & - & - \\
\hline 2 & 500 & 45 & 4.34 & 10.4 & 4.34 & 100,091 & $24.82 \cdot 10^{6}$ & 0.62 & - & - & - \\
\hline 3 & 500 & 25 & 7.80 & 3.2 & 27.80 & 99,938 & $7.65 \cdot 10^{6}$ & 1.95 & - & - & - \\
\hline
\end{tabular}

The calculations that are performed in chapters B.III and B.IV are checked with the three-dimensional potential theory program DELFRAC. The hydrodynamic behaviour of structures 1,2 and 3 from table B-1 will be calculated with the calculation model as it is described in this appendix and DELFRAC. 


\section{B.II Waves}

\section{B.II.a Linear potential theory of waves}

The potential theory is used to derive the velocity potential of waves. Together with the boundary conditions, the velocity potential theory determines the wave characteristics such as the relation between wave height, wave length, water depth and the particle motions within the wave.

The wave characteristics and the hydraulic boundary conditions determine the motions of the floating breakwater. In the linear potential theory, all flows are considered to be incompressible, irrotational, non-viscous, continuous and homogenous.

The velocity potential $\Phi(x, y, z)$ is given as a scalar function at each point in the fluid. The derivatives of the velocity potential yield the velocity of a water particle in a specific direction.

The potential theory holds for regular, harmonic, sinus-shaped wave profiles. The velocity and thus the motion of a water particle depend on its distance below the mean water level. The wave potential can be written as:

$\Phi(x, y, z)=P(z) \cdot \sin (k x-\omega t)$

Where:

$P(z) \quad: \quad$ A function that describes the velocity distribution over the depth.

The boundary conditions that restrict the wave potential of equation 6.6 in order to find a more specific definition of $\mathrm{P}(\mathrm{z})$ are:

1. Continuity condition or Laplace equation

2. Sea bed boundary condition

3. Free surface dynamic boundary condition

4. Free surface kinematic boundary condition

Ad.1 The continuity or Laplace equation hypothesizes the incompressibility of the fluid. A change of potential in one of the directions should in this case immediately lead into an opposite change of potential in the other directions.

$\nabla^{2} \Phi_{w}=\frac{\partial^{2} \Phi_{w}}{\partial x^{2}}+\frac{\partial^{2} \Phi_{w}}{\partial y^{2}}+\frac{\partial^{2} \Phi_{w}}{\partial z^{2}}=0$

Ad.2 Interchange of vertical energy at the seabed is considered to be impossible. The vertical velocity of water particles at the seabed is therefore zero (no-leak condition).

$$
\frac{\delta \Phi}{\delta z}=0 \quad \text { for: } \mathrm{z}=-\mathrm{h}
$$

Since deep-water conditions are considered in this report and deep-water waves are not influenced by bottom friction, equation 6.8 is expanded to:

$$
\frac{\delta \Phi}{\delta z}=\frac{\delta \Phi}{\delta x}=\frac{\delta \Phi}{\delta y}=0 \quad \text { for: } \mathrm{z}=-\mathrm{h}
$$


Ad.3 The pressure at the free water surface $(z=\zeta)$ is equal to the atmospheric pressure. Since waves with a relative small steepness are considered, the Bernoulli equation for an unstationary irrotational flow becomes:

$$
\frac{\delta \Phi}{\delta t}+\frac{p}{\rho}+g \zeta=0
$$

After some algebra ${ }^{1}$, the wave potential in deep water conditions, depending on the water depth, becomes:

$$
\Phi_{w}(t)=\frac{\zeta_{a} g}{\omega} \cdot e^{k z} \cdot \sin (k x-\omega t)
$$

Ad.4 The vertical velocity of a water particle at the free surface of the fluid is identical to the vertical velocity of the fluid itself. This leads to:

$$
\begin{array}{ll}
\frac{\delta \Phi_{w}}{\delta z}=\frac{\delta \zeta}{\delta t} & \text { for } \mathrm{z}=0 \\
\frac{\partial^{2} \Phi}{\partial t^{2}}+g \frac{\delta \Phi}{\delta z}=0 & \text { for: } \mathrm{z}=0
\end{array}
$$

In chapter 6.1.2 the boundary conditions which involve the presence of the floating breakwater are discussed.

\footnotetext{
${ }^{1}$ Journée, J.M.J. and Massie, W.W. 'Offshore Hydromechanics' $1^{\text {st }}$ edition, Delft University of Technology.
} 


\section{B.II.b Water particle kinematics}

Figure B-2 shows the distribution of the particle velocity over the water depth. A combination of the dispersion relation (eq.5.5) and the influence of the water depth on the wave potential (eq.B-5) yield the development of the particle velocity over the depth:

$$
\begin{aligned}
& \frac{\partial \Phi_{w}}{\partial z}=\dot{z}=\omega \cdot \zeta_{a} \cdot e^{-k z} \cdot \sin (\omega t) \\
& \frac{\partial \Phi_{w}}{\partial y}=\dot{y}=\omega \cdot \zeta_{a} \cdot e^{-k z} \cdot \cos (\omega t)
\end{aligned}
$$

The horizontal and vertical velocities have a phase difference of $1 / 2 \pi$ to one another.

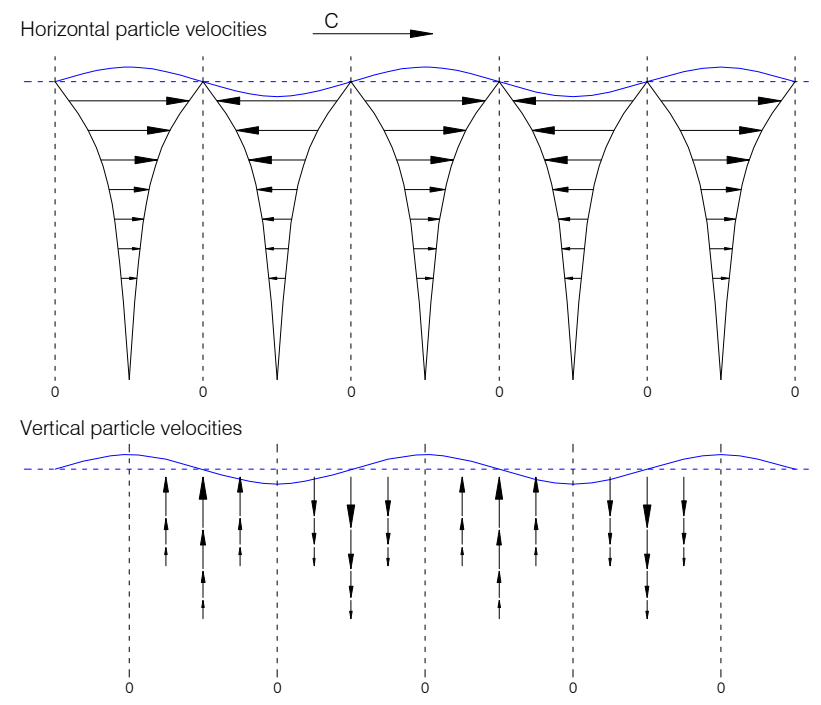

Figure B-2: Particle velocities in deep water waves.

Due to the fact that deep water waves are not influenced by the bottom of the sea, the trajectories of the particles become circular:

$$
\left(x-x_{1}\right)^{2}+\left(z-z_{1}\right)^{2}=\left(\zeta_{a} \cdot e^{k z_{1}}\right)^{2}
$$




\section{B.II.c Water pressure}

The total water pressure in deep water conditions at a depth z consists of the linearized Bernoulli pressure and the dynamic wave pressure.

$$
p=\underbrace{-\rho g z}_{\text {Linear }}+\underbrace{\rho g \zeta_{a} \cdot e^{k z} \cdot \cos (k x-\omega t)}_{\text {Dynamic }}
$$

\section{B.II.d Wave energy}

To attenuate the wave height to a level, acceptable to perform harbour activities, the floating breakwater has to deal with the amount of energy present in the wave. The total energy per wave length is calculated by a summation of the kinetic and the potential wave energy. This total wave energy is described by ${ }^{1}$ :

$$
E_{t o t}=\frac{1}{2} \rho g \zeta_{a}^{2}
$$

As the radii of the trajectories of wave particles decrease exponentially with the depth in deep water environments, the same happens with the energy level of the wave. The energy distribution over the depth can be described with:

$$
E_{Z}=e^{-k z} \cdot \frac{1}{2} \rho g \zeta_{a}^{2}
$$

The energy influencing factors of the floating breakwater are:

$\begin{array}{lll}E_{a} & : & \text { Incoming wave energy } \\ E_{R} & : & \text { Reflected wave energy } \\ E_{U} & : & \text { Transmitted wave energy due to underflow } \\ E_{C} & : & \text { Radiated wave energy due to structural motions } \\ E_{D} & : & \text { Energy reduction due to structural behaviour. } \\ E_{A} & : & \text { Energy absorption by the structure. }\end{array}$

With these factors, the energy balance of a single wave becomes: $E_{a}=E_{R}+E_{U}+2 \cdot E_{C}+E_{D}+E_{A}$

The energy level at the harbour side of the floating breakwater: $E_{H}=E_{U}+E_{C}$

The energy level at the ocean side of the floating breakwater:

$$
E_{S}=E_{a}+E_{C}+E_{R}
$$

$$
\begin{array}{ll}
= & \frac{1}{2} \rho g \zeta_{a}^{2} \\
= & \frac{1}{2} \rho g \zeta_{R}^{2} \\
= & e^{-k z} \cdot \frac{1}{2} \rho g \zeta_{a}^{2} \\
= & \frac{1}{2} \rho g \zeta_{r a d}^{2} \\
= & \Delta E
\end{array}
$$

\footnotetext{
${ }^{1}$ Prof.dr.ir.J.A.Battjes, 'Short waves', Delft University of Technology.
} 


\section{B.III Forces and Moments}

During the calculations that will be performed, a clear distinction should be made between the wave exciting forces and moments and the hydromechanical forces and moments. Wave exciting forces and moments are produced by the waves coming in on the floating breakwater. Hydromechanical forces and moments are produced by the motion of the floating breakwater it self.

\begin{tabular}{|c|c|c|c|}
\hline Incoming wave & $\begin{array}{c}\text { Motion of the floating } \\
\text { breakwater }\end{array}$ & $\begin{array}{c}\text { Transmitted wave due to } \\
\text { breakwater motions and } \\
\text { underflow }\end{array}$ & $\begin{array}{l}\text { Vertical motion of the } \\
\text { governing vessel. }\end{array}$ \\
\hline
\end{tabular}

Figure B-3: Wave force-structural motion relation

Figure B-3 shows that there is a relation between these two forces and motions in the case of the floating breakwater. Because of the incoming wave forces, the structure will be put into a certain motion. This motion itself will generate waves on both sides of the structure that will propagate away from the structure.

The summation of both the wave $\left(\mathrm{X}_{\mathrm{w}}\right)$ - and the hydromechanical $\left(\mathrm{X}_{\mathrm{h}}\right)$ forces and moments constitute the total forces on the structure. For the six degrees of freedom in the three-dimensional case these forces and moments become:

\begin{tabular}{|c|c|c|c|c|}
\hline Surge & : & $\frac{d}{d t}(\rho \nabla \cdot \dot{x})$ & $=\rho \nabla \cdot \ddot{x}$ & $=X_{h 1}+X_{w 1}$ \\
\hline Sway & : & $\frac{d}{d t}(\rho \nabla \cdot \dot{y})$ & $=\rho \nabla \cdot \ddot{y}$ & $=X_{h 2}+X_{w 2}$ \\
\hline Heave & : & $\frac{d}{d t}(\rho \nabla \cdot \dot{z})$ & $=\rho \nabla \cdot \ddot{z}$ & $=X_{h 3}+X_{w 3}$ \\
\hline Roll & : & $\frac{d}{d t}\left(I_{x x} \cdot \dot{\phi}-I_{x z} \cdot \dot{\psi}\right)$ & $=I_{x x} \cdot \ddot{\phi}-I_{x z} \cdot \ddot{\psi}$ & $=X_{h 4}+X_{w 4}$ \\
\hline Pitch & : & $\frac{d}{d t}\left(I_{y y} \cdot \dot{\theta}\right)$ & $=I_{y y} \cdot \ddot{\theta}$ & $=X_{h 5}+X_{w 5}$ \\
\hline Yaw & : & $\frac{d}{d t}\left(I_{z z} \cdot \dot{\psi}-I_{z x} \cdot \dot{\phi}\right)$ & $=I_{z z} \cdot \ddot{\psi}-I_{z x} \cdot \ddot{\phi}$ & $=X_{h 6}+X_{w 6}$ \\
\hline
\end{tabular}

In the next section, a 2-dimensional calculation will be performed in order to determine the influence of several parameters on the behaviour of the floating breakwater. In this simplified, 2-dimensional model, the 3 degrees of freedom which are treated are: sway, heave and roll.

\section{Strip theory}

The strip theory is developed to calculate the forces on- and motions of a three dimensional floating body. The method uses the uncoupled two-dimensional hydromechanical and wave loads to determine the three dimensional behaviour.

The two-dimensional representation of the hydromechanical- and wave loads are according to this theory:

$$
\begin{aligned}
& X_{h j}^{\prime}=\frac{D}{D t}\left\{A_{i j}^{\prime} \cdot \ddot{\zeta}_{h j}^{*}\right\}+B_{i j}^{\prime} \cdot \dot{\zeta}_{h j}^{*}+X_{r s j}^{\prime} \\
& X_{w j}^{\prime}=\frac{D}{D t}\left\{A_{i j}^{\prime} \cdot \ddot{\zeta}_{w j}^{*}\right\}+B_{i j}^{\prime} \cdot \dot{\zeta}_{w j}^{*}+X_{f k j}^{\prime}
\end{aligned}
$$

Where:
$X_{h j}^{\prime} \quad:$
The uncoupled two-dimensional potential hydromechanical loads.
$X_{w j}^{\prime} \quad:$
$A_{i j}^{\prime} \quad:$
The uncoupled two-dimensional potential wave loads.
$B_{i j}^{\prime}$
The two-dimensional potential mass.
$X_{r s j} \quad:$
$X_{f k j} \quad:$
$\dot{\zeta}_{h j}^{*} \quad: \quad$ Velocity of the water particles in direction j. 
Derived for the two-dimensional motions, equations B.13-14 become:

\section{Hydromechanical loads}

Sway $: X_{h 2}^{\prime}=A_{h 22}^{\prime} \cdot \ddot{\zeta}_{h 2}^{*}+B_{h 22}^{\prime} \cdot \dot{\zeta}_{h 2}^{*}$

Heave $: X_{h 3}^{\prime}=A_{h 33}^{\prime} \cdot \ddot{\zeta}_{h 3}^{*}+B_{h 33}^{\prime} \cdot \dot{\zeta}_{h 3}^{*}+C^{\prime}{ }_{h 33} \cdot \zeta_{h 3}^{*}$

Roll $\quad: X_{h 4}^{\prime}=A_{h 44}^{\prime} \cdot \ddot{\zeta}_{h 4}^{*}+B_{h 44}^{\prime} \cdot \dot{\zeta}_{h 4}^{*}+C_{h 44}^{\prime} \cdot \zeta_{h 4}^{*}$

Wave loads

Sway $\quad: X_{w 2}^{\prime}=A_{22}^{\prime} \cdot \ddot{\zeta}_{w 2}^{*}+B_{22}^{\prime} \cdot \dot{\zeta}_{w 2}^{*}$

Heave $: X_{w 3}^{\prime}=A_{33}^{\prime} \cdot \ddot{\zeta}_{w 3}^{*}+B_{33}^{\prime} \cdot \dot{\zeta}_{w 3}^{*}+C^{\prime}{ }_{33} \cdot \zeta^{*}{ }_{w 3}$

Roll $\quad: X_{w 4}^{\prime}=A_{44}^{\prime} \cdot \ddot{\zeta}_{w 4}^{*}+B_{44}^{\prime} \cdot \dot{\zeta}_{w 4}^{*}+C_{44}^{\prime} \cdot \zeta_{w 4}^{*}$

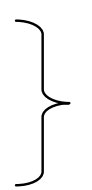

Where:

$C_{i j}^{\prime} \quad: \quad$ The Froude-Krilov coefficient, written as a spring term.

Combining formulae B.12,B.15 and B.16, the uncoupled equations of motion becomes:

\section{Sway equation of motion:}

$$
\left(\rho \nabla+a_{22}\right) \cdot \ddot{y}+\left(d_{b y}+b_{22}\right) \cdot \dot{y}+\left(k_{c y}\right) \cdot y=a_{22} \cdot \ddot{\zeta}_{w 2}^{*}+b_{22} \cdot \dot{\zeta}_{w 2}^{*}
$$

\section{Heave equation of motion:}

$$
\left(\rho \nabla+a_{33}\right) \cdot \ddot{z}+\left(d_{b z}+b_{33}\right) \cdot \dot{z}+\left(k_{c z}+c_{33}\right) \cdot z=a_{33} \cdot \ddot{\zeta}_{w 3}^{*}+b_{33} \cdot \dot{\zeta}_{w 3}^{*}+c_{33} \cdot \zeta_{w 3}^{*} \cdot C_{3}
$$

\section{Roll equation of motion:}

$$
\left(I_{x x}+a_{44}\right) \cdot \ddot{\phi}+\left(d_{b \phi}+b_{44}\right) \cdot \dot{\phi}+\left(k_{b \phi}+c_{44}\right) \cdot \phi=a_{44} \cdot \ddot{\zeta}_{w 4}^{*}+b_{44} \cdot \dot{\zeta}_{w 4}^{*}+c_{44} \cdot \zeta_{w 4}^{*}
$$

Where:

$\begin{array}{ll}y & : \\ \dot{y} & : \\ \ddot{y} & : \\ z & : \\ \dot{z} & : \\ \ddot{z} & : \\ \phi & : \\ \dot{\phi} & : \\ \ddot{\phi} & : \\ \zeta_{w n}^{*} & : \\ \dot{\zeta}_{w n}^{*} & : \\ \ddot{\zeta}_{w n}^{*} & : \\ \nabla & : \\ \rho & : \\ d_{b n} & : \\ k_{c n} & : \\ a_{n n} & : \\ b_{n n} & : \\ c_{n n} & : \\ \mathcal{E}_{n \zeta} & : \\ C_{3} & :\end{array}$

Displacement in the $\mathrm{y}$-direction $(\mathrm{m})$.

Velocity in the $\mathrm{y}$-direction $(\mathrm{m} / \mathrm{s})$.

Acceleration in the $\mathrm{y}$-direction $\left(\mathrm{m} / \mathrm{s}^{2}\right)$.

Displacement in the z-direction (m).

Velocity in the z-direction $(\mathrm{m} / \mathrm{s})$.

Acceleration in the $\mathrm{z}$-direction $\left(\mathrm{m} / \mathrm{s}^{2}\right)$.

Displacement in the $\phi$-direction (rad).

Velocity in the $\phi$-direction $(\mathrm{rad} / \mathrm{s})$.

Acceleration in the $\phi$-direction $\left(\mathrm{rad} / \mathrm{s}^{2}\right)$.

Reduced wave elevation in the n-direction.

Reduced velocity of the water particles in the n-direction.

Reduced acceleration of the water particles in the n-direction.

Volume of water displacement of the body in an equilibrium state $\left(\mathrm{m}^{3}\right)$.

Density of the water $\left(\mathrm{kg} / \mathrm{m}^{3}\right)$.

Mooring damping in $\mathrm{n}$-direction $(\mathrm{Ns} / \mathrm{m})$.

Mooring spring stiffness in $n$-direction $(\mathrm{N} / \mathrm{m})$.

Hydrodynamic mass coefficient $\left(\mathrm{Ns}^{2} / \mathrm{m}\right)$

Hydrodynamic damping coefficient $(\mathrm{Ns} / \mathrm{m})$

Restoring spring coefficient $(\mathrm{N} / \mathrm{m})$

Phase shift.

Vertical wave pressure distribution coefficient

$$
\begin{aligned}
& =-y_{a} \cos \left(\omega t+\varepsilon_{y \zeta}\right) \\
& =y_{a} \omega \sin \left(\omega t+\varepsilon_{y \zeta}\right) \\
& =y_{a} \omega^{2} \cos \left(\omega t+\varepsilon_{y \zeta}\right) \\
& =z_{a} \sin \left(\omega t+\varepsilon_{z \zeta}\right) \\
& =z_{a} \omega \cos \left(\omega t+\varepsilon_{z \zeta}\right) \\
& =-z_{a} \omega^{2} \sin \left(\omega t+\varepsilon_{z \zeta}\right) \\
& =-\phi_{a} \cos \left(\omega t+\varepsilon_{\phi \zeta}\right) \\
& =\phi_{a} \omega \sin \left(\omega t+\varepsilon_{\phi \zeta}\right) \\
& =\phi_{a} \omega^{2} \cos \left(\omega t+\varepsilon_{\phi \zeta}\right)
\end{aligned}
$$

The wave parameters $\zeta_{w n}^{*}, \dot{\zeta}_{w n}^{*}$ and $\ddot{\zeta}_{w n}^{*}$ are called 'reduced' because of the draft of the structure (see fig.6.7 in the report). 


\section{B.IV Hydrodynamic calculation parameters}

In formulae B.17-19, $\mathrm{a}_{\mathrm{ij}}$, $\mathrm{b}_{\mathrm{ij}}$ and $\mathrm{c}_{\mathrm{ij}}$ are hydrodynamic coefficients. $\mathrm{a}_{\mathrm{ij}}$ and $\mathrm{b}_{\mathrm{ij}}$ depend on the structural shape and the frequency of motion, while $\mathrm{c}_{\mathrm{ij}}$ is a coefficient that depends on the shape only.

The equations of motion, as they were presented in equations B.17-19, have the appearance of a set of linear differential equations of the second order. In the form as they are presented here, they are analogous to the equations for the mechanical systems as a pendulum or a mass-spring system. Few similarities are obtained however, when the mass and damping coefficients are considered. Unlike the pendulum and the mass-spring system, the coefficients $\mathrm{a}_{\mathrm{ij}}$ and $\mathrm{b}_{\mathrm{ij}}$ are not constant but functions of the frequency of motion.

The magnitude of coefficients $a_{i j}$ and $b_{i j}$ is determined by the hydrodynamic reaction as a result of the movement of the floating breakwater with respect to the water. However, since the hydrodynamic reaction itself is determined by the coefficients $a_{i j}$, $b_{i j}$ and $c_{i j}$, it is almost impossible to state the magnitude of the coefficients $\mathrm{a}_{\mathrm{ij}}$, and $\mathrm{b}_{\mathrm{ii}}$.

In his PhD thesis, Vugts ${ }^{1}$ performed experiments to state the magnitude of coefficients $a_{i j}$, and $b_{i i}$ at several motion frequencies for three structural width/draft $\left(\mathrm{Yw} / \mathrm{T}_{1}\right)$ relations. The results of Vugts will be used in this report to determine the wave transmission.

Vugts did experimental research to find the added mass and damping parameters for three different rectangular cross sections $\left(\mathrm{Yw} / \mathrm{T}_{1}=2,4\right.$ and 8$)$. From these measurements, polynomials will be derived to approximate the hydrodynamic parameters over the whole frequency range. The hydrodynamic polynomials of constructions with width/draft ratios between 2 and 8 are estimated by iteration.

Constructions with a large width relative to the draft will become a problem in this case, since no test results are available. To overcome this problem, the hydrodynamic values of a structure with a width/draft ratio of 10.4 are estimated, based on the known measured data from Vugts.

To state the reliability, these estimates are compared with the values, used by the three dimensional potential theory computer model DELFRAC.

\section{Screen influence}

The installation of a screen underneath the structure will influence the hydrodynamic parameters of the sway and roll motions. This phenomenon was not examined by Vugts. In his book, Newman ${ }^{2}$ provides added mass coefficients for two-dimensional submerged bodies, including plates.

Since the floating breakwater consists of a floating body with a submerged vertical plate underneath it, a combination of the Vugts and Newman parameters was used to estimate the added mass of the floating breakwater. The obtained values are checked with DELFRAC and, if necessary, corrected.

The hydrodynamic sway- and roll damping parameters of the floating breakwater with screen are determined totally with DELFRAC.

\section{The submerged area of the cylinder section}

Since the model, as it is used in this research, applies the hydrodynamic parameters measured by Vugts, an important remark has to be made on the submerged area of the cylinder section A. According to Vugts, $\mathrm{A}$ is the part of the structure that is underneath the waterline when it is floating in still water.

One has to keep in mind that Vugts did his measurements by oscillating a model with an external force. This is the reason why the coefficient is not influenced by the amplitude of motion, since this amplitude is kept constant for the whole frequency range. A constant amplitude of motion results in a constant amplitude of the water displacing area $\left(\mathrm{A}_{\mathrm{s}}\right)$.

\footnotetext{
${ }^{1}$ Vugts, J.H. (1970). PhD thesis 'The hydrodynamic forces and ship motions in waves', Delft University of Technology.

${ }^{2}$ Newman J.N. (1977). 'Marine Hydrodynamics'. MIT Press, Cambridge, United Kingdom
} 
Since the amplitude of motion of the floating breakwater depends on the exciting wave force distribution, the water displacing area will depend on this factor too. Thus, the water displacing area, as it is used by Vugts needs a correction to make it suitable for a wave- driven model.

The water displacing area used in the measured data by Vugts is:

$$
A=Y_{w} \cdot T_{1}
$$

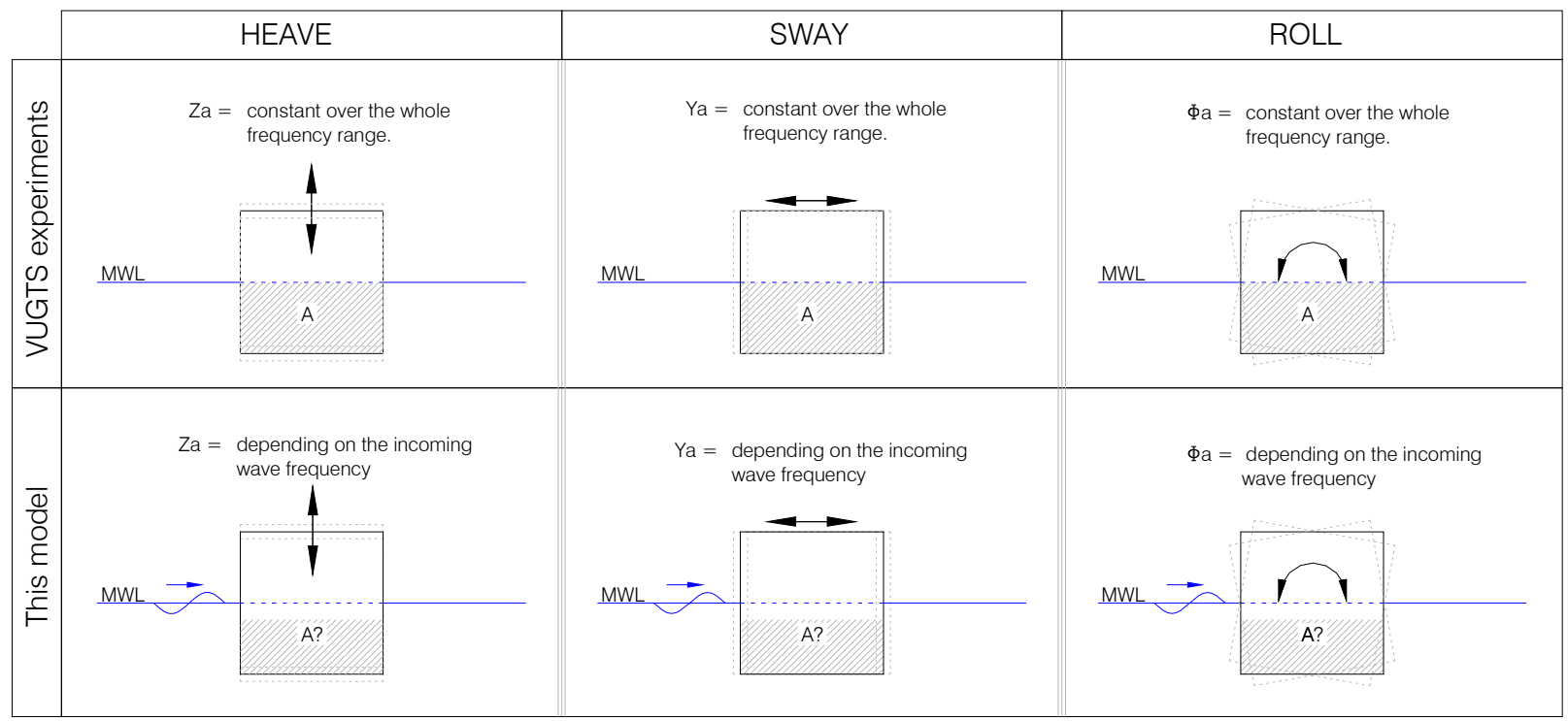

Figure B-4: Differences in determining 'A' between a external loaded structure and a wave loaded structure

The water displacing area of the floating breakwater becomes:

$$
A_{w}=Y_{w} \cdot T_{1} \cdot L_{w}
$$




\section{B.IV.aHydrodynamic mass}

The hydrodynamic - or added mass $\left(\mathrm{a}_{\mathrm{ij}}\right)$ is a coefficient that describes the amount of water $(\mathrm{kg})$ that has to be put into motion when the floating breakwater is moved. This coefficient depends on the shape of the structure (width, draft) as well as the frequency of motion.

The added mass is schematically shown in figure B-5 for the three two-dimensional motions.

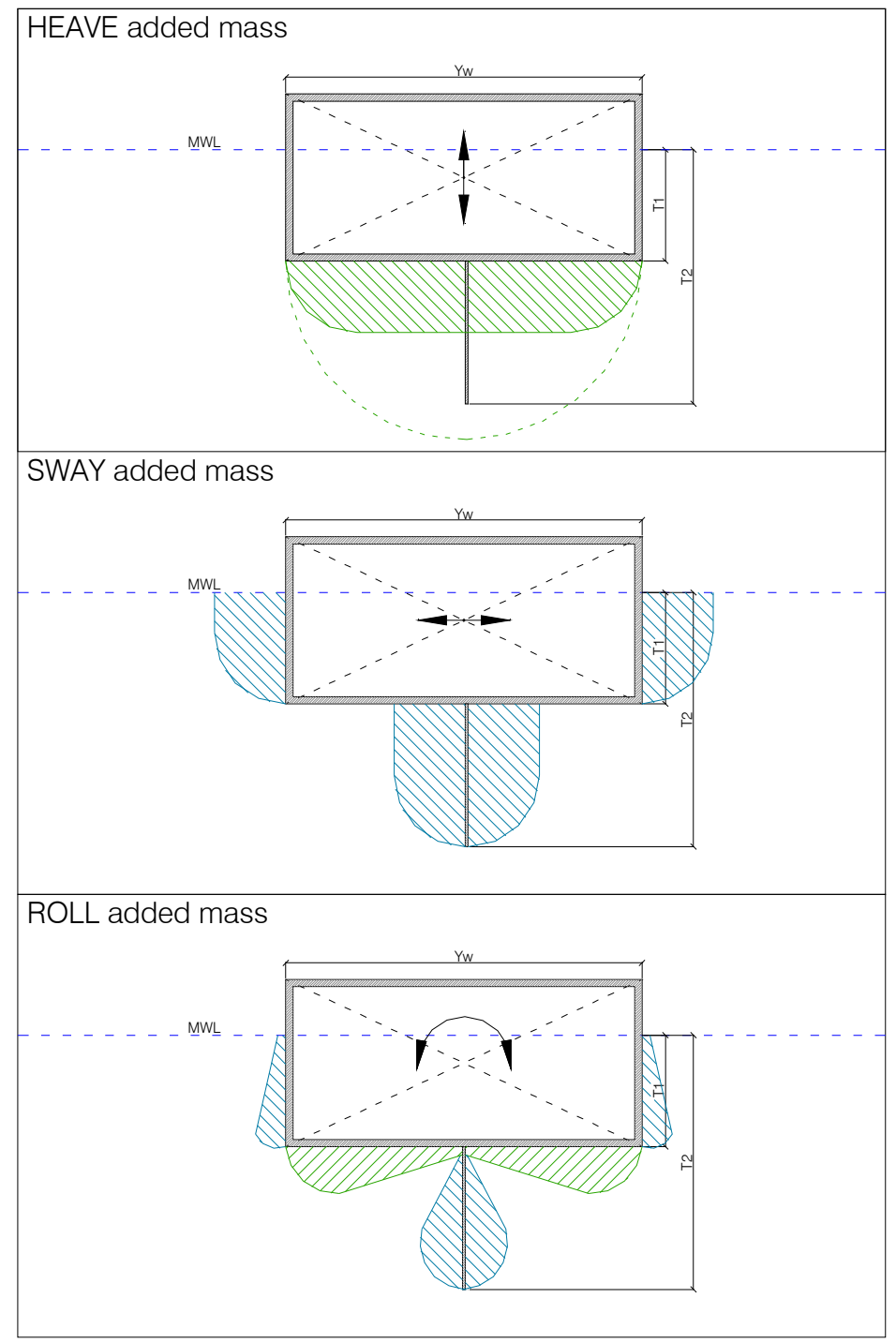

Figure B-5: Added mass for heave, sway and roll. 


\section{B.IV.a.1 Heave added mass}

The added mass that has been measured by Vugts for heaving, two-dimensional rectangular structures with with/draft relations of $\mathrm{Yw}_{\mathrm{w}} / \mathrm{T}_{1}: 2,4$ and 8 is shown in figure B-6.
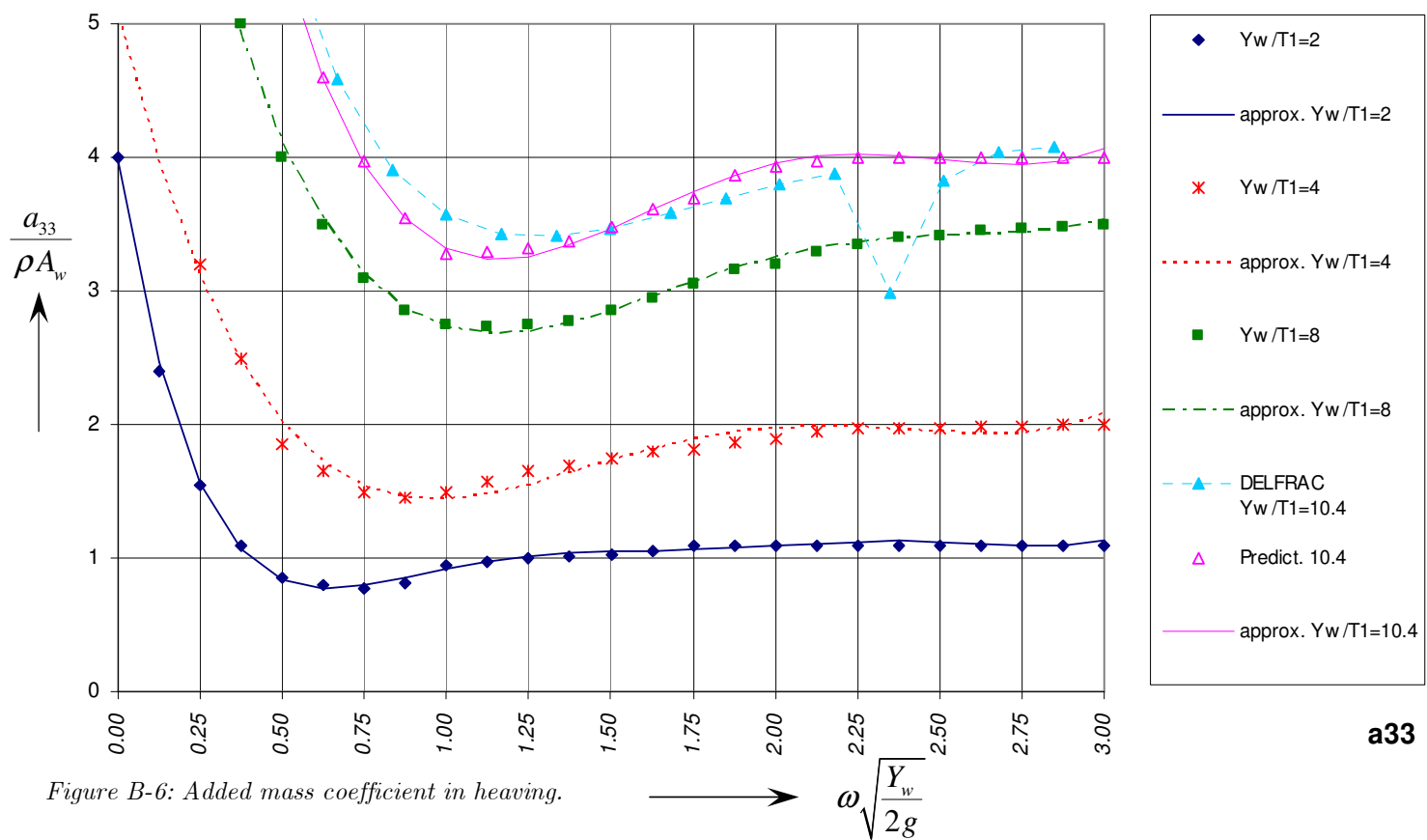

The prediction, based on the Vugts measurements for a width/draft ratio of 10.4 is reflected in figure B-6 as well. This estimate of the added mass parameters is close to the DELFRAC calculations. The irregularity, visible in the DELFRAC calculations of figure B-6 is due to the so-called irregular frequencies. Irregular frequencies are associated with eigenfrequencies of the internal (non-physical) flow of the body. At these frequencies the Green function has no unique solution for the internal problem and as a result the exterior solution is also affected at these points.

The added mass polynomials, as they are used during this research are summarized in the table below.

\begin{tabular}{c|c|}
\hline $\mathrm{Yw}_{\mathrm{w}} \mathrm{T}_{1}$ & HEAVE added mass Polynomials \\
\hline$\leq 2.0$ & $0.2232 \mathrm{x}^{6}-2.4237 \mathrm{x}^{5}+10.507 \mathrm{x}^{4}-23.097 \mathrm{x}^{3}+26.789 \mathrm{x}^{2}-15.046 \mathrm{x}+3.9672$ \\
\hline 4.0 & $0.4576 \mathrm{x}^{4}-3.5098 \mathrm{x}^{3}+9.4053 \mathrm{x}^{2}-9.9766 \mathrm{x}+5.0659$ \\
\hline 8.0 & $0.4791 \mathrm{x}^{4}-4.039 \mathrm{x}^{3}+12.135 \mathrm{x}^{2}-14.794 \mathrm{x}+8.9469$ \\
\hline$\geq 10.4$ & $0.8382 \mathrm{x}^{4}-6.8965 \mathrm{x}^{3}+20.164 \mathrm{x}^{2}-24.154 \mathrm{x}+13.367$ \\
\hline \multicolumn{2}{|c}{ Table B-2: Added mass polynomials for heaving structures. }
\end{tabular}

Where:

$$
\begin{array}{llll}
x & : & \omega \sqrt{\frac{Y_{w}}{2 g}} & \\
a_{33}^{\prime}: & \frac{a_{33}}{\rho A_{w}} & \\
A_{w} & : & Y_{w} \cdot T_{1} \cdot L_{w} & \text { submerged area of the breakwater section }
\end{array}
$$


The values for heave added mass calculated by DELFRAC and by this model, show some differences for constructions with a large constructional width. This difference only occurs when the width/draft ratio is larger than 8. A good agreement is gained when the constructional width/draft ratios are between 2 and 8 , as is shown in figure B-7 for a structure with a width/draft ratio of 3.2 .

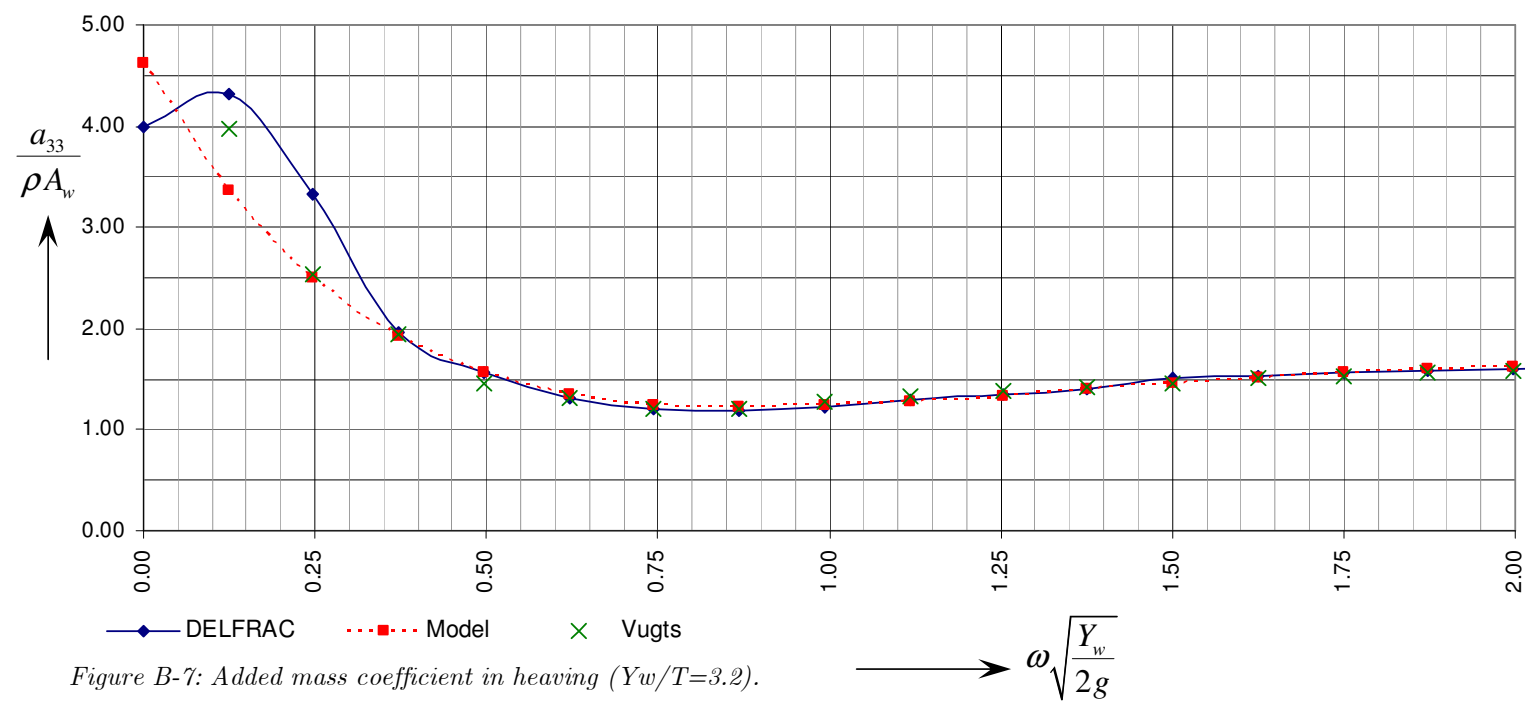




\section{B.IV.a.2 Sway added mass}

The added mass that has been measured by Vugts for the sway motion of two-dimensional rectangular structures with with/draft relations of $\mathrm{Yw} / \mathrm{T}_{1}: 2,4$ and 8 is shown in figure $\mathrm{B}-8$.
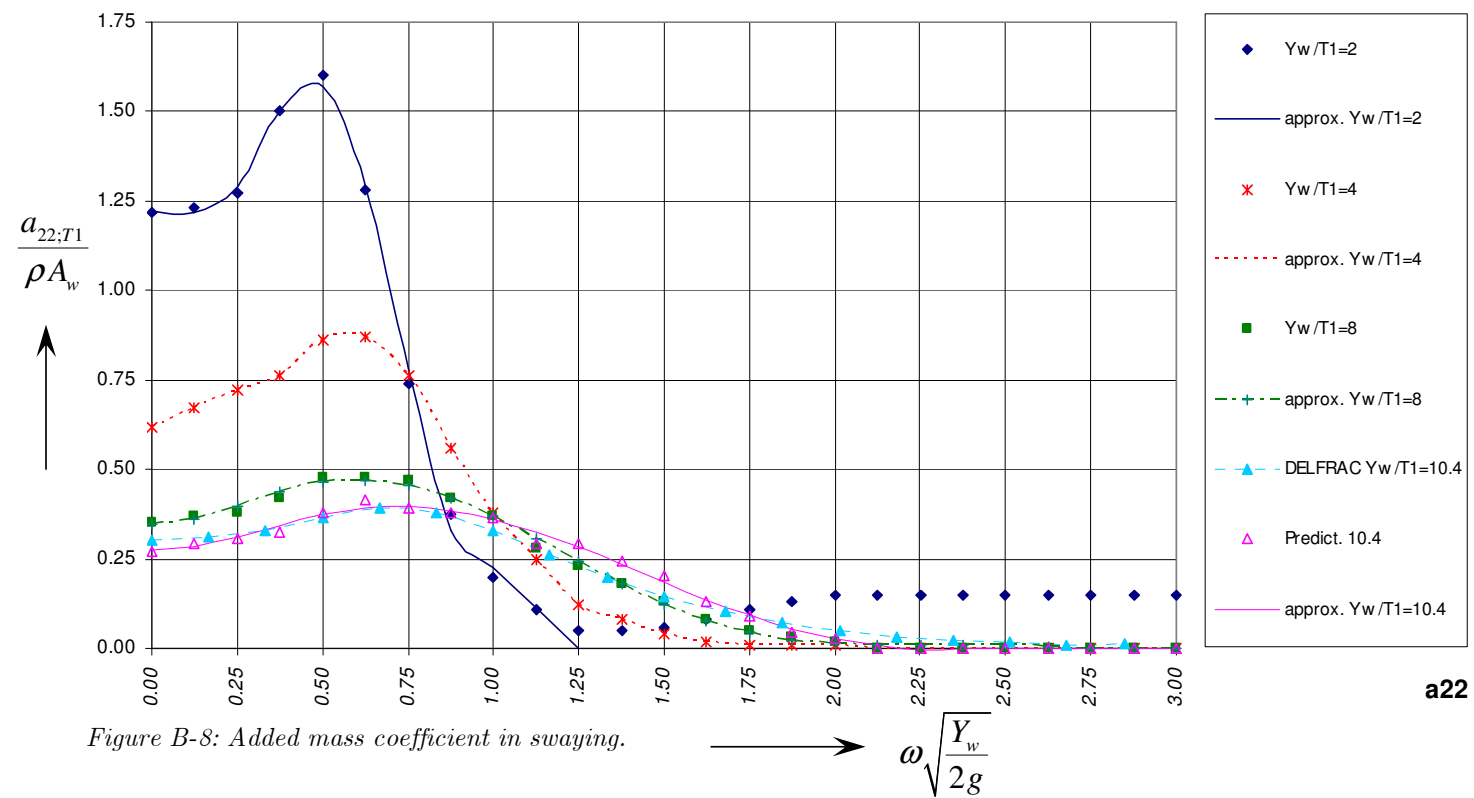

The estimate of the added mass, based on the Vugts measurements, for a structure with a width/draft ration of 10.4 is reflected in figure B- 8 as well. The estimate of the sway added mass parameters is close to the DELFRAC calculations.

The added mass polynomials, as they are used during this research are summarized in the table below.

\begin{tabular}{c|c}
\hline $\mathrm{Yw}_{\mathrm{w}} / \mathrm{T}_{1}$ & SWAY added mass Polynomials (no screen) \\
\hline$\leq 2.0$ & $-89.02 x^{6}+286.1 x^{5}-325.86 x^{4}+153.26 x^{3}-26.971 x^{2}+1.4943 x+1.2228$ \\
\hline 4.0 & $-15.656 x^{6}+60.148 x^{5}-83.506 x^{4}+49.757 x^{3}-12.39 x^{2}+1.4031 x+0.6184$ \\
\hline 8.0 & $0.0681 x^{6}-0.6521 x^{5}+2.3022 x^{4}-3.5158 x^{3}+1.9143 x^{2}-0.0973 x+0.3487$ \\
\hline$\geq 10.4$ & $0.0185 x^{6}-0.225 x^{5}+0.9861 x^{4}-1.836 x^{3}+1.1965 x^{2}-0.0534 x+0.2746$ \\
\hline Table B-3: Added mass polynomials for swaying structures.
\end{tabular}

Where:

$\begin{array}{lll}x & : & \omega \sqrt{\frac{Y_{w}}{2 g}} \\ a_{22}^{\prime} & : & \frac{a_{22 ; T 1}}{\rho A_{w}}\end{array}$

$A_{w} \quad: \quad Y_{w} \cdot T_{1} \cdot L_{w} \quad$ Water displacing area of the underwater cylinder section 
The prediction, based on the Vugts measurements is similar to the DELFRAC calculation when a structure with width/draft ratio of 10.4 is considered. Figure B-9 shows that the DELFRAC calculations do agree with the Vugts-based estimates as well when width/draft ratios between 2 and 8 are considered (3.2 in this example).

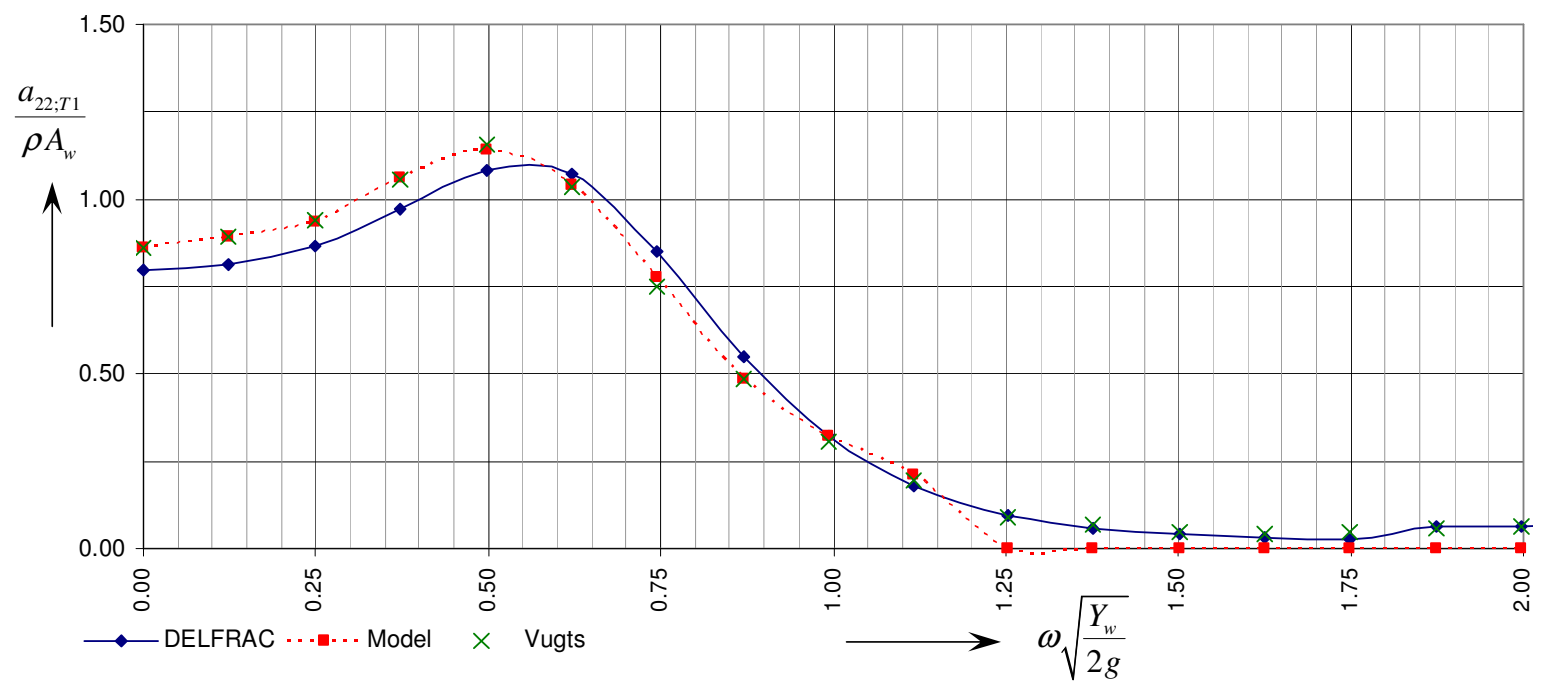

Figure B-9: Added mass coefficient in swaying ( $Y w / T=3.2)$. 


\section{Screen influence}

The influence of a screen underneath the floating breakwater on the sway added mass is determined in this section. The sway added mass consists of the added mass contribution of the floating part, determined with the Vugts ${ }^{1}$ measurements, and the added mass contribution of the submerged vertical screen, determined with Newmans' ${ }^{2}$ formula. This combination leads to:

$$
a_{22}=a_{22}^{\prime} \cdot \rho A_{w}+\rho \cdot \pi \cdot L_{w}\left(1 / 2 \cdot\left(T_{2}-T_{1}\right)\right)^{2}
$$

Where:

$T_{1} \quad$ : $\quad$ Draft of the floating section

$T_{2} \quad$ : $\quad$ Screen draft

When the graph of equation (B.21) and the sway added mass according to DELFRAC are compared, only small differences are visible for high-frequency motions (fig B-10). Large difference is visible in the low frequency area.

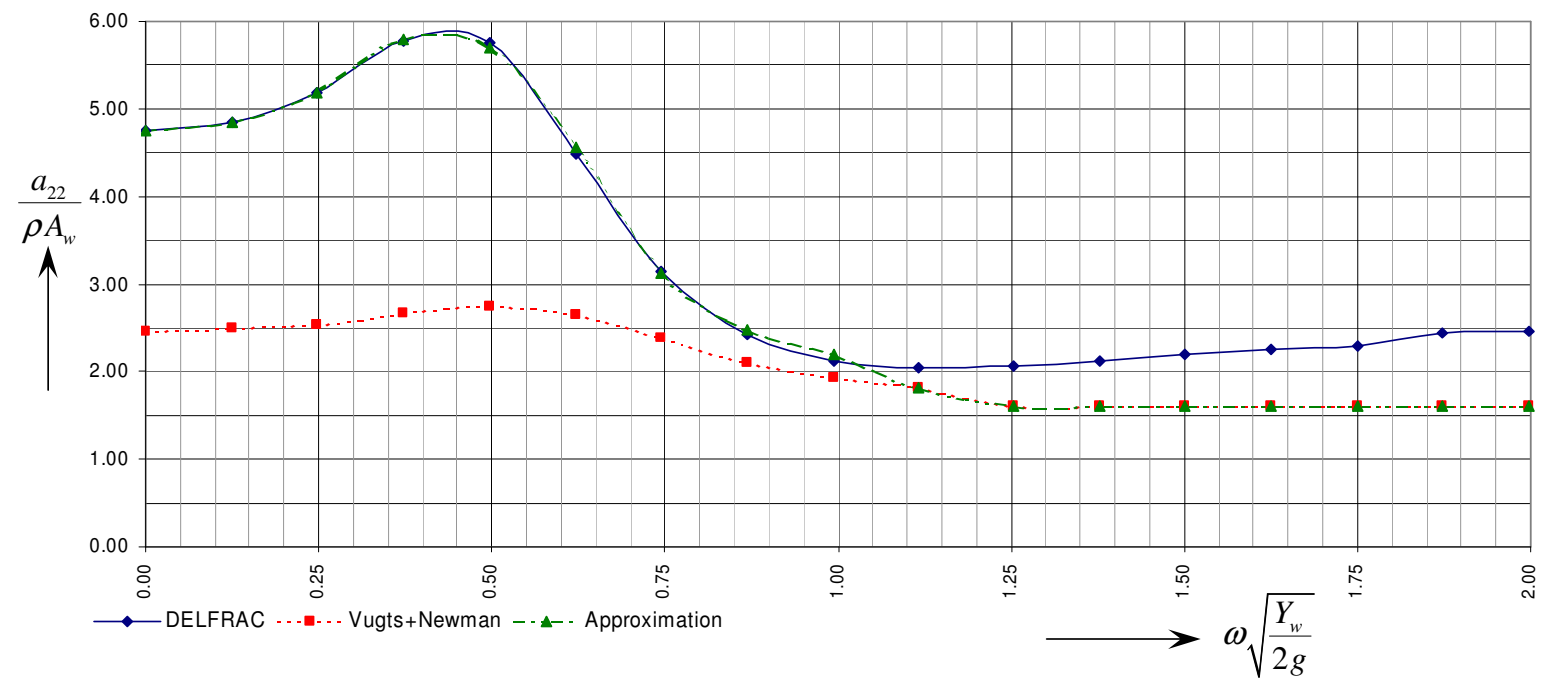

Figure B-10: Added mass coefficient in swaying $\left(Y w / T_{1}=3.2\right.$, screen length $\left.=20 \mathrm{~m}\right)$.

An additional polynomial is used to approximate the influence of a screen underneath the floating body for low-frequency motions. This polynomial is determined for both a screen length of 10 meters and $20 \mathrm{~m}$. The additional sway added mass polynomials, as they are used during this research to state the contribution of the screen at low-frequency motions, are summarized in the table below

\begin{tabular}{l|l}
\hline Screen length $(\mathrm{m})$ & SWAY added mass Additional Polynomials $\left(\mathrm{P}_{\mathrm{s} ; \mathrm{a} ; \mathrm{a}}\right)$ \\
\hline 10 & \\
\hline 20 & $-396.31 x^{6}+1124.7 x^{5}-1130.4 x^{4}+469.66 x^{3}-74 x^{2}+4.2714 x+2.2808$ \\
\hline Table B-4: Additional added mass polynomials for structures with a screen underneath it.
\end{tabular}

The total sway added mass equation for a rectangular floating breakwater with a screen underneath the floating part now becomes:

$a_{22}=\left(a_{22}^{\prime}+P_{s ; a ; a}\right) \cdot \rho A+\rho \cdot \pi \cdot L_{w}\left(1 / 2 \cdot\left(T_{2}-T_{1}\right)\right)^{2}$

Where:

$P_{s ; a ; a} \quad: \quad$ The sway added mass additional polynomial.

${ }^{1}$ Vugts, J.H. (1970). PhD thesis 'The hydrodynamic forces and ship motions in waves', Delft University of Technology.

${ }^{2}$ Newman J.N. (1977). 'Marine Hydrodynamics'. MIT Press, Cambridge, United Kingdom 


\section{B.IV.a.3 Roll added mass moment of inertia}

The roll added mass moment of inertia will be completely determined with DELFRAC. Due to the fact that Vugts ${ }^{1}$ did his tests with fixed roll angles, these measurements can not be used in this case. The measurements will be used as a control tool to check the DELFRAC results.

The graphs of the roll added mass moment of inertia for two constructional lay-outs are shown in figure B-11.

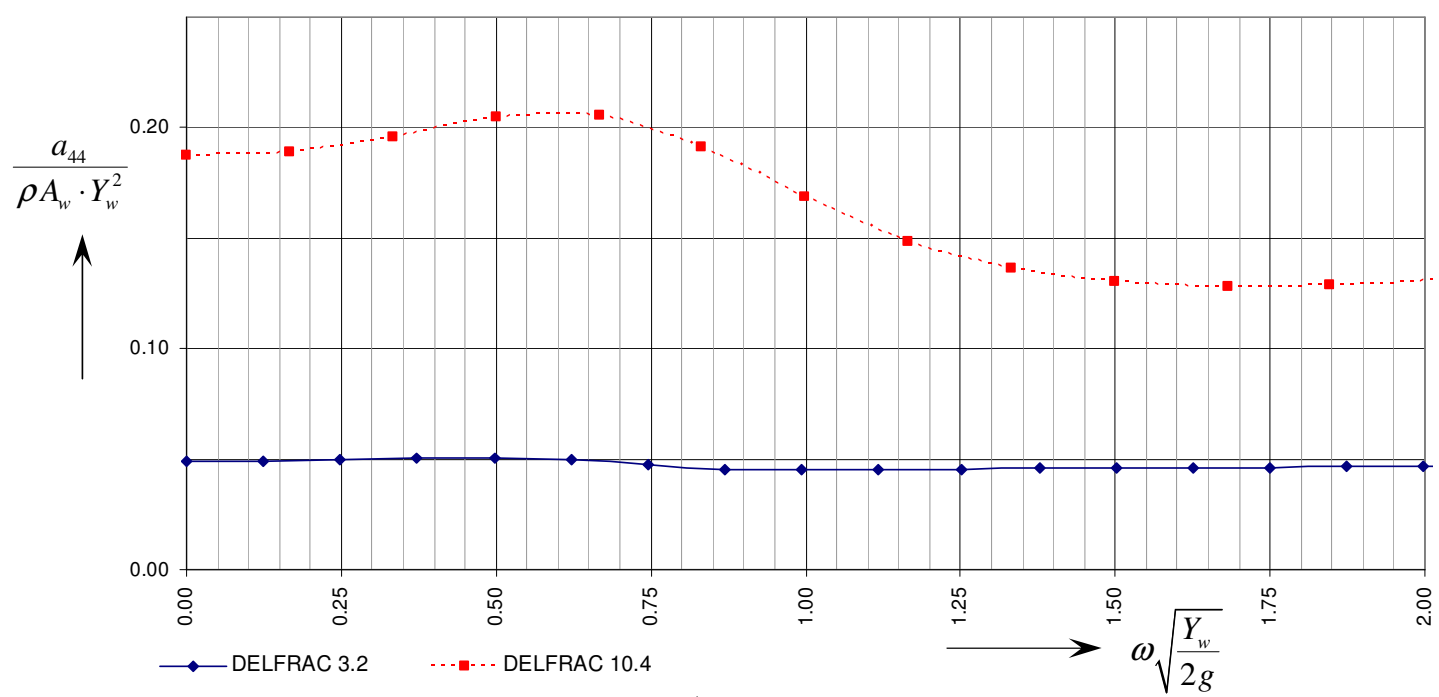

Figure B-11: Roll added mass moment of inertia for $Y_{w} / T_{1}=3.2$ and 10.4 .

The roll added moment of inertia increases when the structural width becomes larger. Figure B-11 confirms this logical statement. The added mass moment of inertia polynomials, as they are used during this research are summarized in the table below.

\begin{tabular}{l|l}
\hline $\mathrm{Yw}_{\mathrm{w}} / \mathrm{T}_{1}$ & ROLL added mass moment of inertia Polynomials (no screen) \\
\hline$\leq 3.2$ & $0.0007 x^{3}-0.002 x^{2}-0.0021 x+0.05$ \\
\hline$\geq 10.4$ & $-0.1096 x^{5}+0.56 x^{4}-0.9411 x^{3}+0.5345 x^{2}-0.0601 x+0.1876$ \\
\hline Table B-5: Added mass polynomials for rolling structures.
\end{tabular}

Where:

$\begin{array}{lll}x & : \quad \omega \sqrt{\frac{Y_{w}}{2 g}} \\ a_{44}^{\prime} & : \quad \frac{a_{44}}{\rho A_{w} \cdot Y_{w}^{2}}\end{array}$

$A_{w} \quad: \quad Y_{w} \cdot T_{1} \cdot L_{w} \quad$ Water displacing area of the underwater cylinder section

\footnotetext{
${ }^{1}$ Vugts, J.H. (1970). PhD thesis 'The hydrodynamic forces and ship motions in waves', Delft University of Technology.
} 


\section{Screen influence}

The influence of a screen underneath the floating breakwater on the roll added mass moment of inertia is determined in this section. The roll added mass consists of the added mass contribution of the floating part, determined with the DELFRAC calculations, and the added mass contribution of the submerged vertical screen, determined with Newmans ${ }^{11}$ formula. This combination leads to:

$$
a_{44}=\left(a_{44}^{\prime} \cdot \rho A_{w} \cdot Y_{w}^{2}+\frac{1}{8} \rho \cdot \pi \cdot L_{w} \cdot\left(1 / 2 \cdot\left(T_{2}-T_{1}\right)\right)^{4}\right)
$$

When the graph of equation (B.23) and the sway added mass according to DELFRAC are compared, a deviation is visible over the whole frequency range.

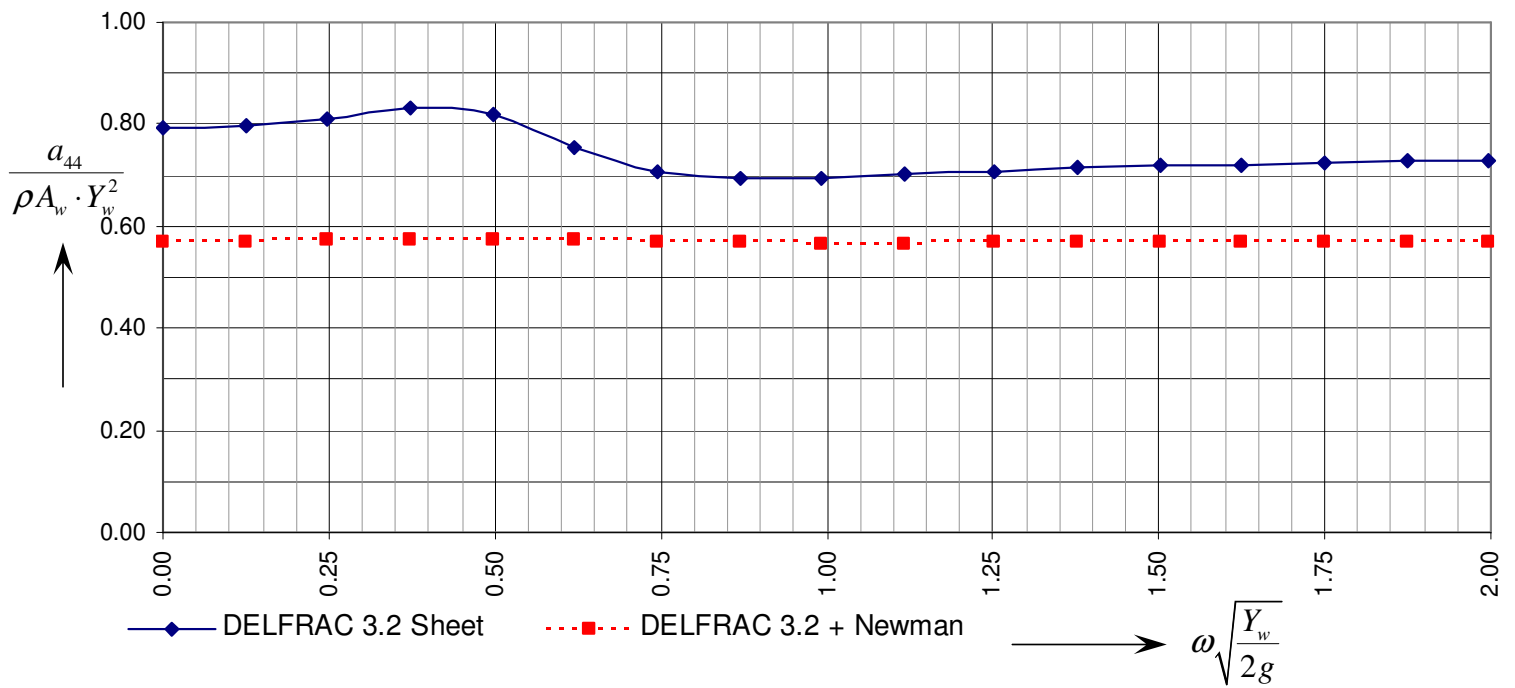

Figure B-12: Added mass moment of inertia in roll $\left(Y w / T_{1}=3.2\right.$, screen length $\left.=20 \mathrm{~m}\right)$.

An additional polynomial is used to approximate the influence of a screen underneath the floating body. This polynomial is determined for both a screen length of 10 meters and $20 \mathrm{~m}$.

The additional roll added mass polynomials, as they are used during this research to state the contribution of the screen, are summarized in the table below

\begin{tabular}{l|l}
\hline Screen length $(\mathrm{m})$ & ROLL added mass Additional Polynomials $\left(\mathrm{P}_{\mathrm{r} ; \mathrm{a} ; \mathrm{a}}\right)$ \\
\hline 10 & \\
\hline 20 & $0.3639 x^{6}-2.0642 x^{5}+4.1249 x^{4}-3.2348 x^{3}+0.5825 x^{2}+0.1322 x+0.2222$ \\
\hline
\end{tabular}

Table B-6: Additional added mass polynomials for structures with a screen underneath it.

The total roll added mass moment of inertia equation for a rectangular floating breakwater with a screen underneath the floating part now becomes:

$a_{44}=\left(a^{\prime}{ }_{44}+\mathrm{P}_{r ; a ; a}\right) \cdot \rho A_{w} \cdot Y_{w}^{2}+\frac{1}{16} \rho \pi \cdot L_{w} \cdot T_{2}^{4}$

The presence of a screen underneath the structure has a large influence on the roll added mass moment of inertia. For a structure with a width/draft ratio of $3.2, a_{44}^{\prime}$ is about 0.05 , as was shown in figure B-11. Installing a $20 \mathrm{~m}$ long screen underneath the same structure, results in a $a_{44}^{\prime}$ of 0.80 .

\footnotetext{
${ }^{1}$ Newman J.N. (1977). 'Marine Hydrodynamics'. MIT Press, Cambridge, United Kingdom
} 


\section{B.IV.bHydrodynamic damping}

The hydrodynamic damping $\left(\mathrm{b}_{\mathrm{ij}}\right)$ is a coefficient that describes the resistance $(\mathrm{kg} / \mathrm{s})$ that the floating body encounters when it is put into motion. Due to the hydrodynamic damping, energy is dissipated from the oscillating structure. This energy radiates away from the structure due to the waves that are produced by the structural motion. The hydrodynamic damping depends on the shape of the oscillating structure as well as the frequency of motion.

\section{B.IV.b.1 Heave damping}

The hydrodynamic damping has been measured by Vugts ${ }^{1}$ for heaving, two-dimensional rectangular structures. He did his measurements for three structures with width/draft ratios of Yw/T: 2,4 and 8 . The results are reflected in figure B-13.

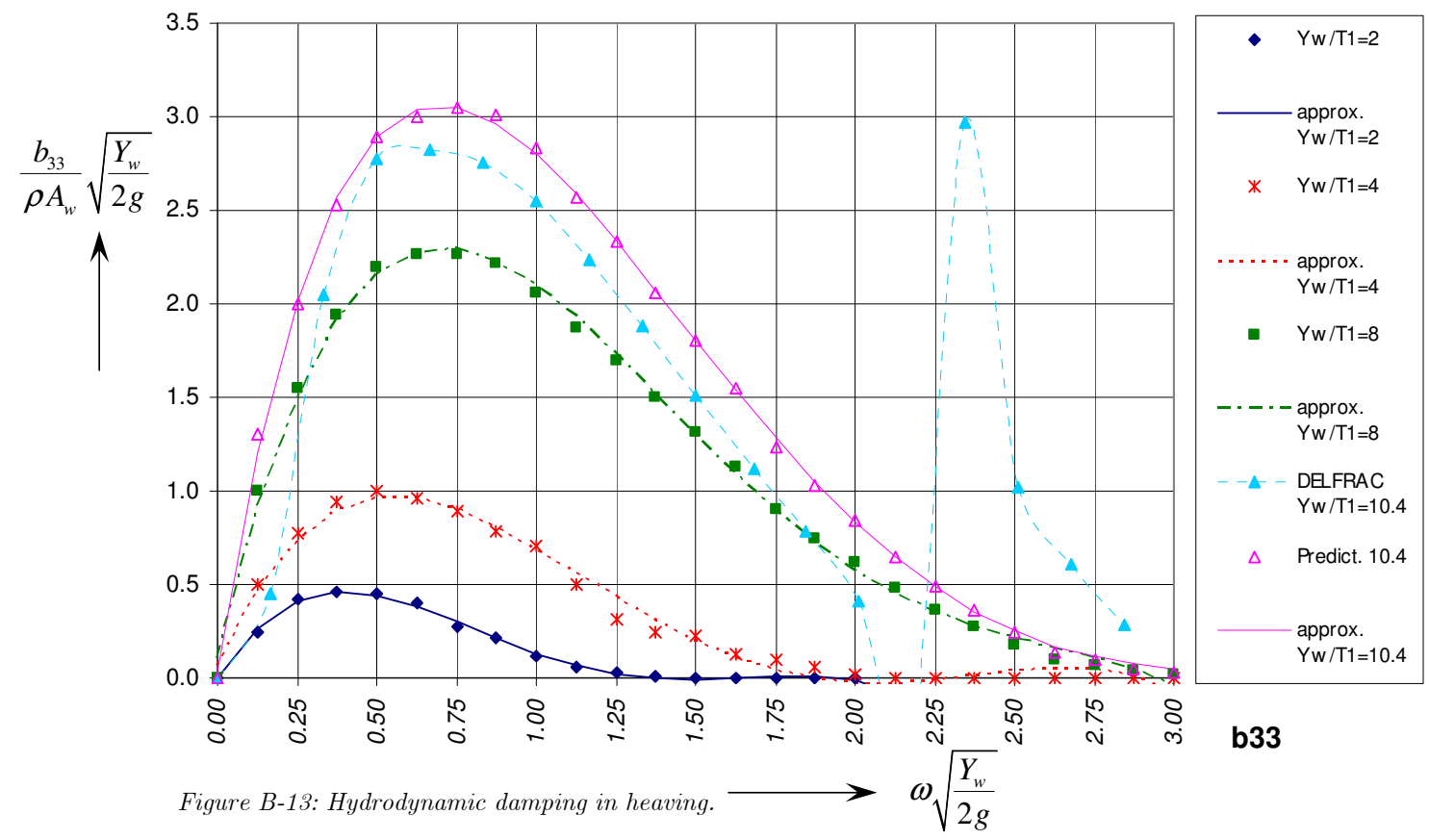

The estimate, based on the Vugts measurements, is reflected in figure B-13 as well. Although this estimate of the hydrodynamic damping parameters is close to the DELFRAC calculations, a deviation is visible over the whole frequency range.

The hydrodynamic damping polynomials, as they are used during this research, are listed in the table below.

\begin{tabular}{c|l}
\hline $\mathrm{Yw}_{\mathrm{w}} / \mathrm{T}_{1}$ & HEAVE hydrodynamic damping Polynomials \\
\hline$\leq 2.0$ & $-0.6402 \mathrm{x}^{4}+3.1564 \mathrm{x}^{3}-5.128 \mathrm{x}^{2}+2.7476 \mathrm{x}-0.0039$ \\
\hline 4.0 & $-0.3121 \mathrm{x}^{4}+2.2045 \mathrm{x}^{3}-5.0934 \mathrm{x}^{2}+3.8185 \mathrm{x}+0.0689$ \\
\hline 8.0 & $-0.36 \mathrm{x}^{4}+2.8995 \mathrm{x}^{3}-7.9383 \mathrm{x}^{2}+7.3908 \mathrm{x}+0.1077$ \\
\hline$\geq 10.4$ & $-0.0237 \mathrm{x}^{6}+0.3108 \mathrm{x}^{5}-1.8936 \mathrm{x}^{4}+6.7088 \mathrm{x}^{3}-13.16 \mathrm{x}^{2}+10.814 \mathrm{x}+0.0435$ \\
\hline
\end{tabular}

Where:

$$
\begin{array}{lll}
x & : & \omega \sqrt{\frac{Y_{w}}{2 g}} \\
b_{33}^{\prime} & : \quad \frac{b_{33}}{\rho A_{w}} \sqrt{\frac{Y_{w}}{2 g}} \\
A_{w} \quad: & Y_{w} \cdot T_{1} \cdot L_{w} \quad \text { Water displacing area of the underwater cylinder section }
\end{array}
$$

\footnotetext{
${ }^{1}$ Vugts, J.H. (1970). PhD thesis 'The hydrodynamic forces and ship motions in waves', Delft University of Technology.
} 
The hydrodynamic damping parameters, used by the computer model DELFRAC and the model as it is used in this report, do show some differences when structures with large width/draft ratios are considered. These deviations are also visible when the structural width/draft ratios are between 2 and 8 , as is shown in figure $\mathrm{B}-14$ for a ratio $\mathrm{Yw} / \mathrm{T}=3.2$.

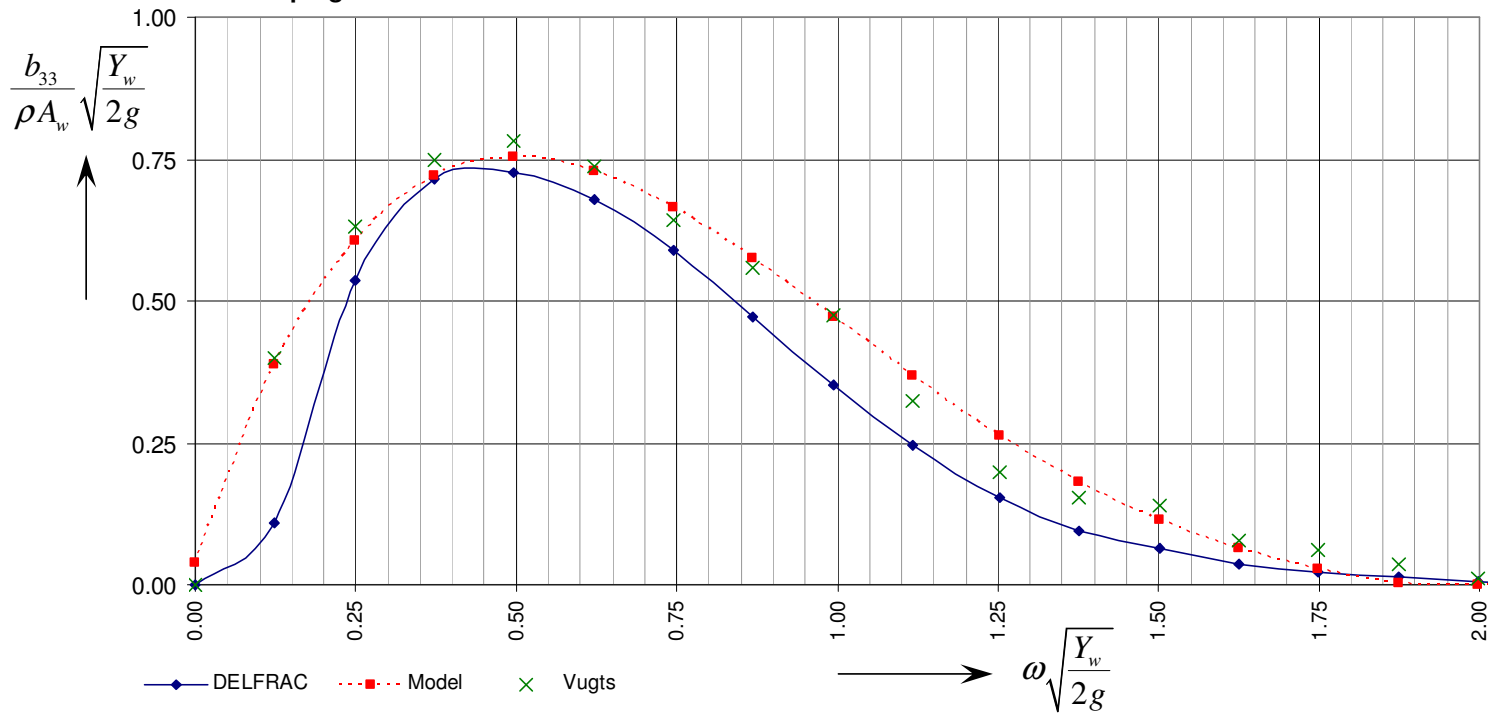

Figure B-14: Hydrodynamic damping coefficient in swaying ( $Y w / T=3.2)$ 


\section{B.IV.b.2 Sway damping}

The hydrodynamic damping has been measured by Vugts $^{1}$ for swaying, two-dimensional rectangular structures. Vugts did his measurements for three structures with width/draft ratios of Yw/T: 2,4 and 8 . The results are reflected in figure B- 15 .

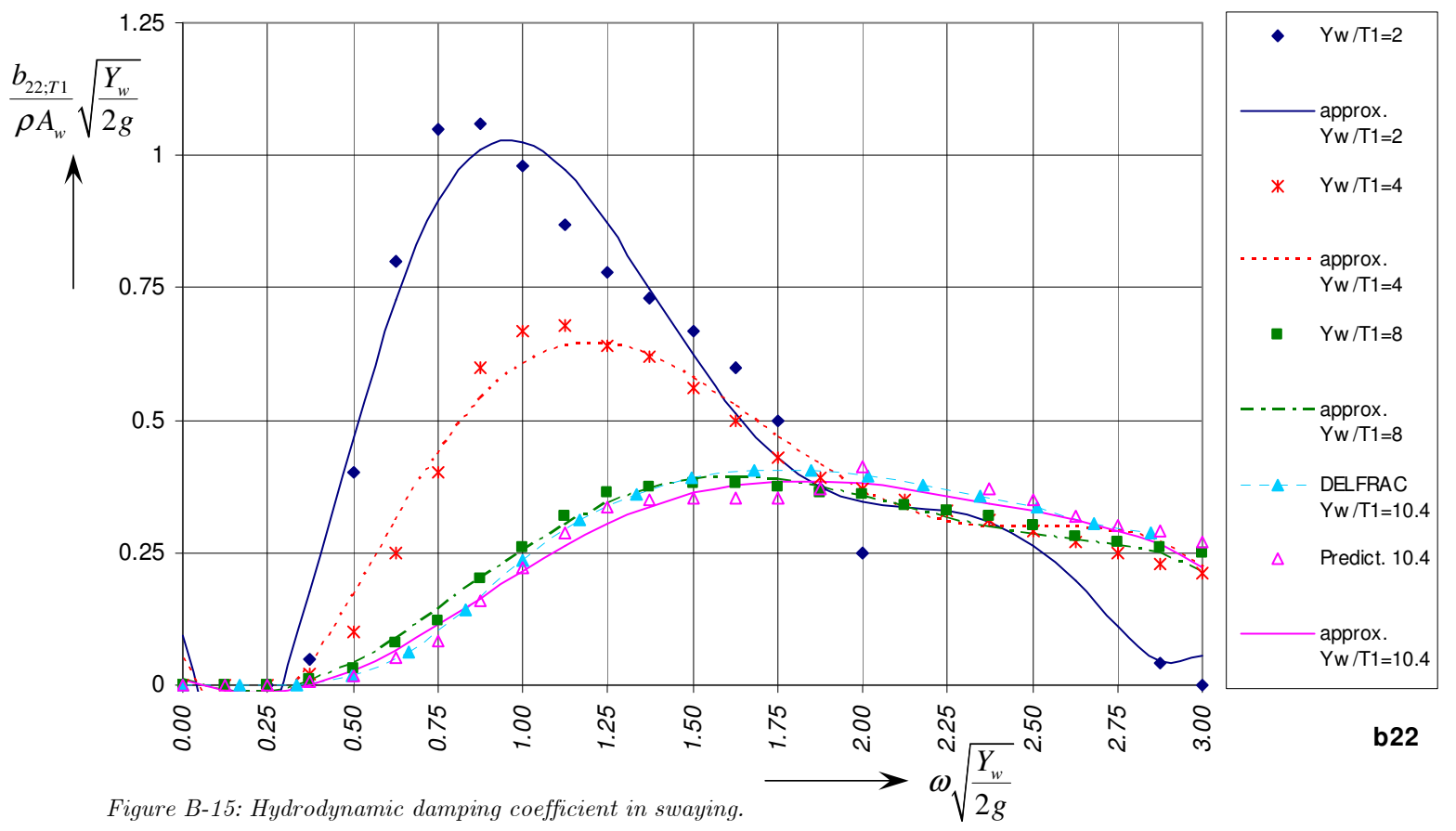

The estimated damping graph for a width/draft ratio of 10.4, based on the Vugts measurements, is reflected in figure B-15 as well. This estimate of the hydrodynamic damping parameters is considerably smaller than the parameters used by DELFRAC over the whole frequency range.

The hydrodynamic damping polynomials, as they are used during this research, are listed in the table below.

\begin{tabular}{c|l}
\hline $\mathrm{Yw} / \mathrm{T}_{1}$ & SWAY hydrodynamic damping Polynomials (no screen) \\
\hline$\leq 2.0$ & $0.3494 \mathrm{x}^{6}-3.4946 \mathrm{x}^{5}+13.292 \mathrm{x}^{4}-23.5 \mathrm{x}^{3}+18.238 \mathrm{x}^{2}-3.9527 \mathrm{x}+0.0945$ \\
\hline 4.0 & $0.0445 \mathrm{x}^{6}-0.6048 \mathrm{x}^{5}+3.0095 \mathrm{x}^{4}-6.7939 \mathrm{x}^{3}+6.6105 \mathrm{x}^{2}-1.7107 \mathrm{x}+0.0518$ \\
\hline 8.0 & $-0.0284 \mathrm{x}^{6}+0.2216 \mathrm{x}^{5}-0.5294 \mathrm{x}^{4}+0.175 \mathrm{x}^{3}+0.6673 \mathrm{x}^{2}-0.2632 \mathrm{x}+0.0107$ \\
\hline$\geq 10.4$ & $-0.0202 \mathrm{x}^{6}+0.1599 \mathrm{x}^{5}-0.3907 \mathrm{x}^{4}+0.1173 \mathrm{x}^{3}+0.5922 \mathrm{x}^{2}-0.2562 \mathrm{x}+0.0118$ \\
\hline \multicolumn{2}{|c|}{ Table B-8: Hydrodynamic damping polynomials for swaying structures. }
\end{tabular}

Where:

$$
\begin{array}{llll}
x & : & \omega \sqrt{\frac{Y_{w}}{2 g}} \\
b_{22}^{\prime} & : & \frac{b_{22 ; T 1}}{\rho A_{w}} \sqrt{\frac{Y_{w}}{2 g}} & \\
A_{w} & : & Y_{w} \cdot T_{1} \cdot L_{w} & \text { Water displacing area of the underwater cylinder section }
\end{array}
$$

The hydrodynamic damping parameters, used by the computer model DELFRAC and the model as it is used in this report, do show a certain deviation when structures with large width/draft ratios are considered. Better similarities are obtained when the structural width/draft ratios are between 2 and 8 , as is shown in figure B-16 for a ratio $\mathrm{Yw} / \mathrm{T}=3.2$.

\footnotetext{
${ }^{1}$ Vugts, J.H. (1970). PhD thesis 'The hydrodynamic forces and ship motions in waves', Delft University of Technology.
} 


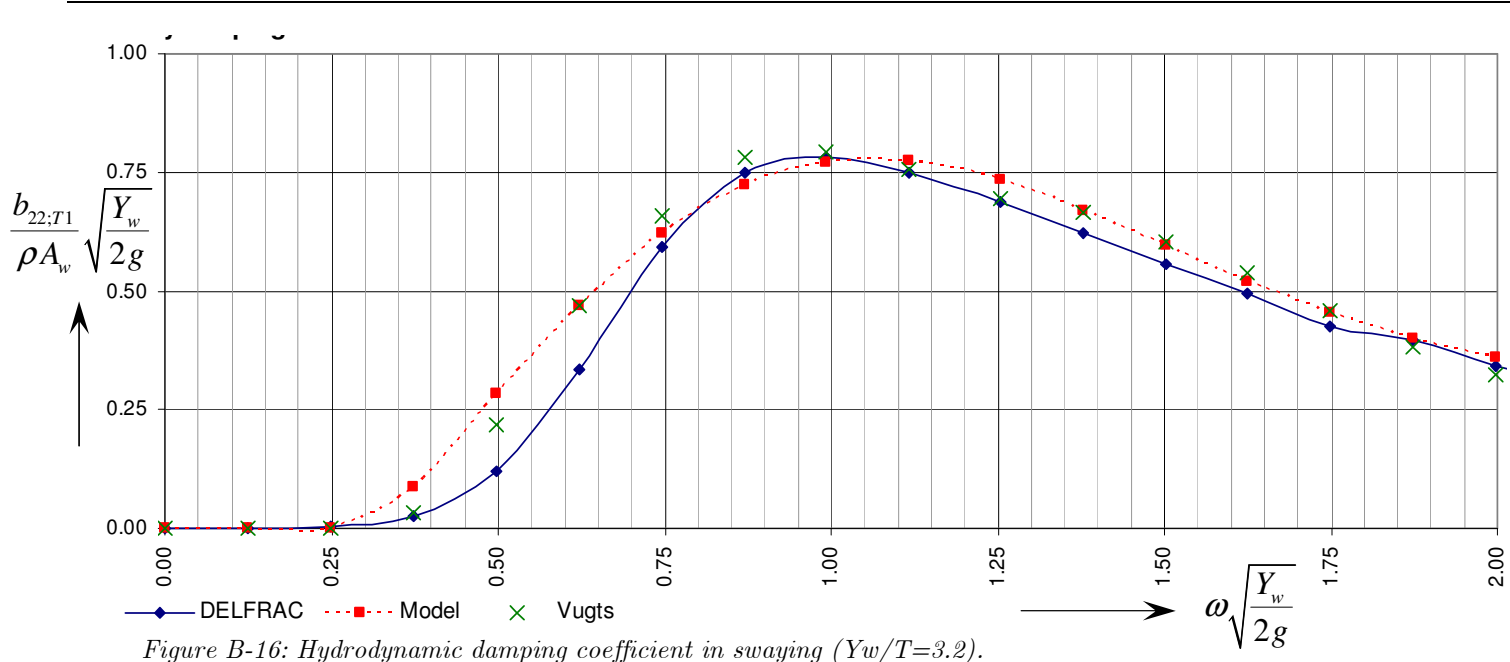

\section{Screen influence}

The influence of a screen underneath the floating breakwater on the sway hydrodynamic damping is determined in this section. Since test results are not available for these kinds of structures, DELFRAC is used to state the equation of the additional polynomial. The influence of a screen is shown in figure B-17.

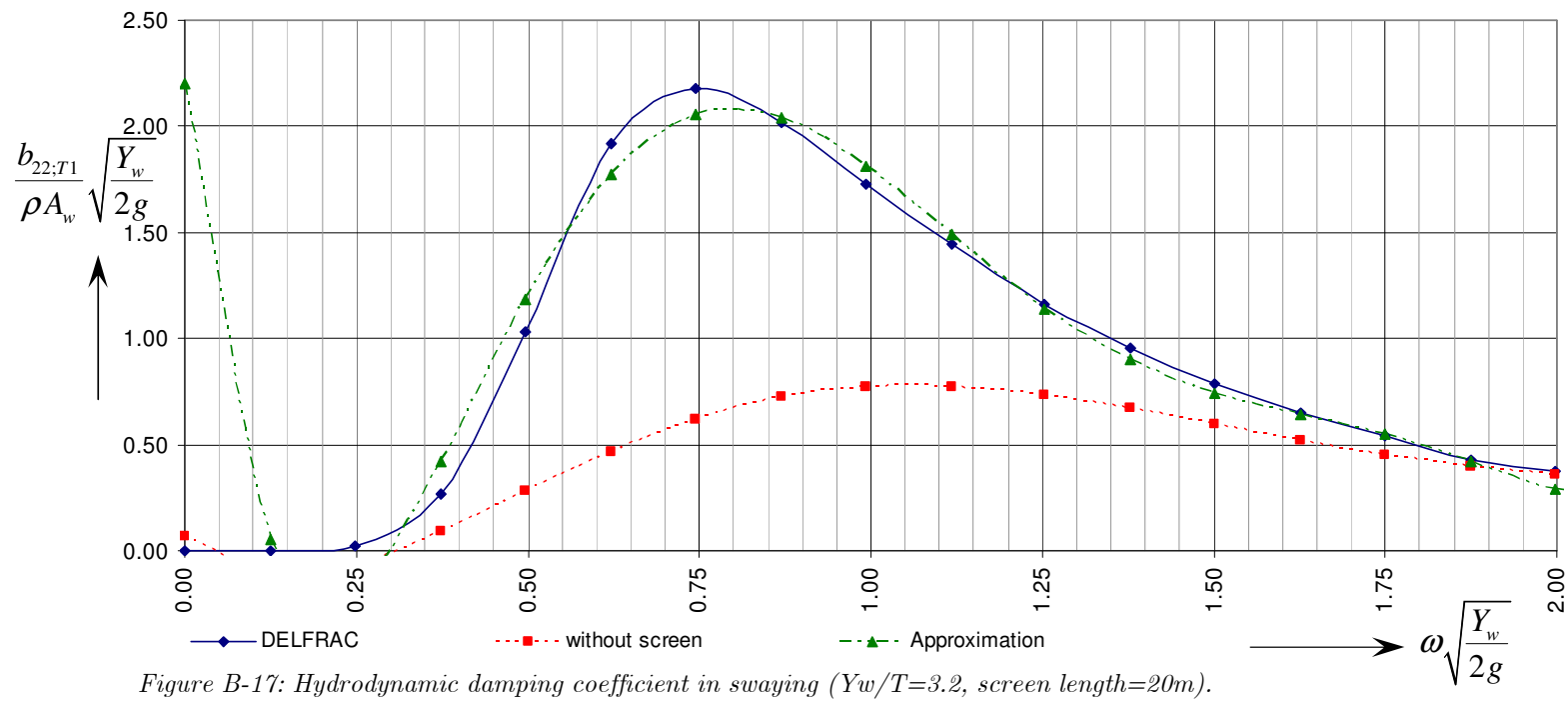

An additional polynomial is used to approximate the influence of a screen underneath the floating body. This polynomial is determined for both a screen length of 10 meters and $20 \mathrm{~m}$.

The additional sway hydrodynamic damping polynomials, as they are used during this research to state the contribution of the screen, are summarized in the table below

\begin{tabular}{l|l}
\hline Screen length $(\mathrm{m})$ & SWAY hydrodynamic damping Additional Polynomials $\left(\mathrm{P}_{\mathrm{s} ; \mathrm{d} ; \mathrm{a}}\right)$ \\
\hline 10 & $2.5245 x^{6}-18.976 x^{5}+55.167 x^{4}-76.831 x^{3}+50.711 x^{2}-13.149 x+1.066$ \\
\hline 20 & $5.0491 x^{6}-37.951 x^{5}+110.33 x^{4}-153.66 x^{3}+101.42 x^{2}-26.298 x+2.1319$ \\
\hline Table B-9: Additional hydrodynamic damping polynomials for structures with a screen underneath it.
\end{tabular}

The total sway hydrodynamic damping equation for a rectangular floating breakwater with a screen underneath the floating part now becomes:

$b_{22}=\left(b_{22}^{\prime}+P_{s ; d ; a}\right) \cdot \frac{\rho A_{w}}{\sqrt{\frac{Y_{w}}{2 g}}}$ 


\section{B.IV.b.3 Roll damping}

The roll damping coefficients, as they were calculated with DELFRAC, are shown in figure B-18.

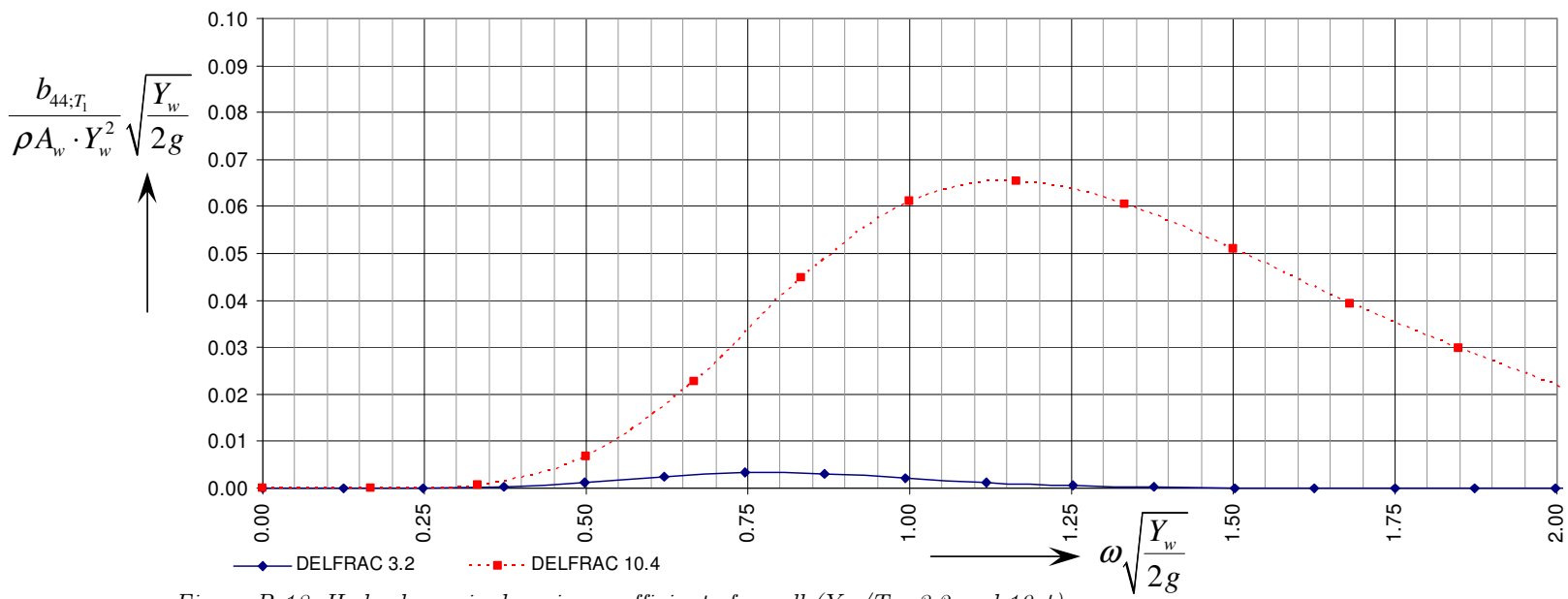

Figure B-18: Hydrodynamic damping coefficients for roll ( $Y w / T_{1}=3.2$ and 10.4).

The hydrodynamic damping polynomials, as they are used during this research, are listed in the table below.

\begin{tabular}{l|c}
\hline $\mathrm{Yw}_{\mathrm{w}} \mathrm{T}_{1}$ & ROLL hydrodynamic damping Polynomials (no screen) \\
\hline$\leq 3.2$ & $-0.0009 x^{6}-0.0133 x^{5}+0.0568 x^{4}-0.0995 x^{3}+0.0708 x^{2}-0.0138 x+0.0003$ \\
\hline$\geq 10.4$ & $-0.0144 x^{6}+0.0999 x^{5}-0.208 x^{4}+0.0547 x^{3}+0.1922 x^{2}-0.0711 x+0.002$ \\
\hline \multicolumn{2}{l}{ Table B-10: Hydrodynamic damping polynomials for rolling structures }
\end{tabular}

Where:

$$
\begin{array}{llll}
x & : & \omega \sqrt{\frac{Y_{w}}{2 g}} \\
b^{\prime}{ }_{44} & : & \frac{b_{44 ; T 1}}{\rho A_{w} \cdot Y_{w}} \sqrt{\frac{Y_{w}}{2 g}} \\
A_{w} & : & Y_{w} \cdot T_{1} \cdot L_{w} & \\
& & \text { Water displacing area of the underwater cylinder section }
\end{array}
$$

\section{Screen influence}

A screen underneath the floating part of the structure has a huge influence on the damping coefficients, as is shown in figure B-19.

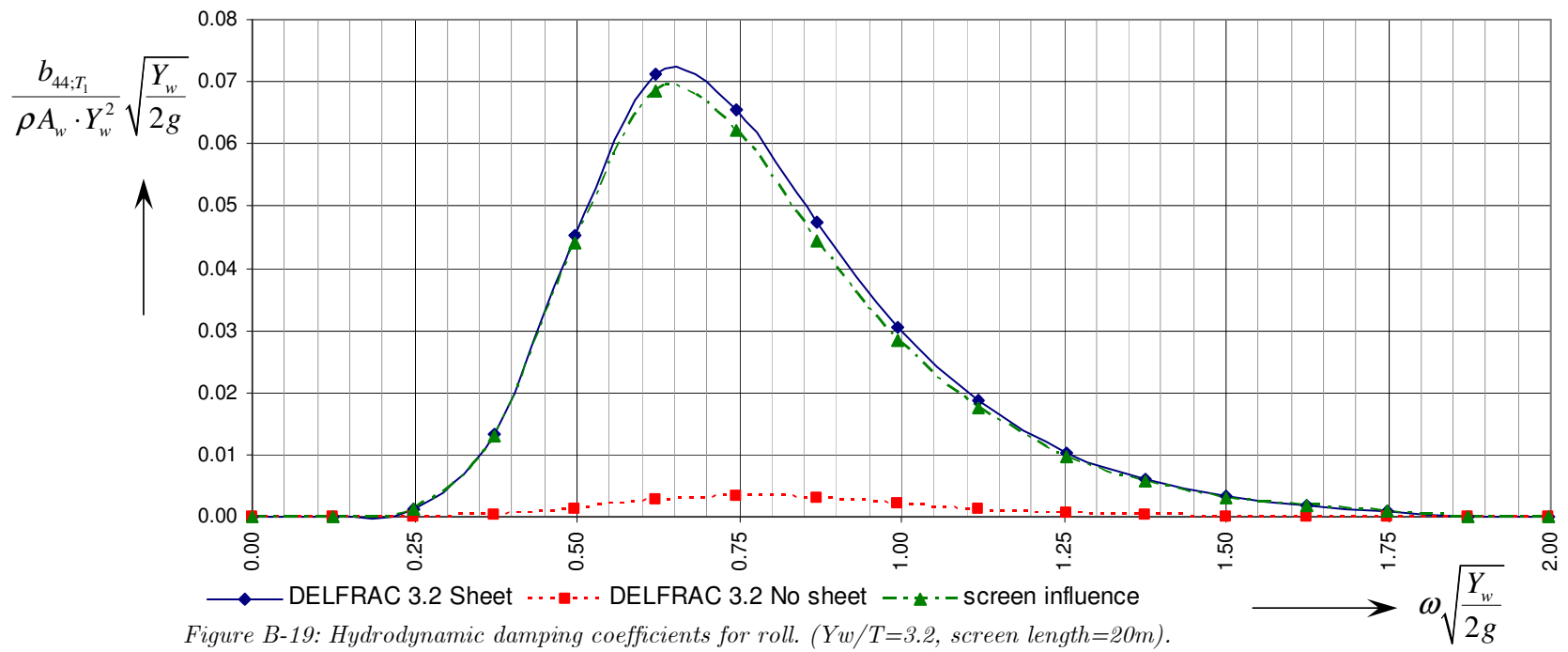


An additional polynomial is used to approximate the influence of a screen underneath the floating body. This polynomial is determined for both a screen length of 10 meters and $20 \mathrm{~m}$.

The additional roll hydrodynamic damping polynomials, as they are used during this research to state the contribution of the screen, are summarized in the table below

\begin{tabular}{l|c|}
\hline Screen length $(\mathrm{m})$ & ROLL hydrodynamic damping Additional Polynomials $\left(\mathrm{P}_{\mathrm{r} ; \mathrm{d} ; \mathrm{a}}\right)$ \\
\hline 10 & $0.0716 x^{6}-0.5283 x^{5}+1.4823 x^{4}-1.9257 x^{3}+1.0902 x^{2}-0.1757 x+0.0022$ \\
\hline 20 & $0.1432 x^{6}-1.0565 x^{5}+2.9646 x^{4}-3.8514 x^{3}+2.1804 x^{2}-0.3513 x+0.0045$ \\
\hline
\end{tabular}

Table B-11: Additional hydrodynamic damping polynomials for structures with a screen underneath it.

The total roll hydrodynamic damping equation for a rectangular floating breakwater with a screen underneath the floating part now becomes:

$b_{44}=\left(b^{\prime}{ }_{44}+P_{r ; d ; a}\right) \cdot \frac{\rho A_{w} \cdot Y_{w}^{2}}{\sqrt{\frac{Y_{w}}{2 g}}}$

(B-26)

Where:

$P_{r ; d ; a} \quad: \quad$ The roll damping additional polynomial 


\section{B.V Structural motions}

The floating breakwater will generate waves from both sides of the structure when it is put into motion by the incoming waves. Figure B-20 gives an overview of the wave properties at both sides (harbour and sea side) of the structure when the motions are considered to be independent and no phase shift is involved.
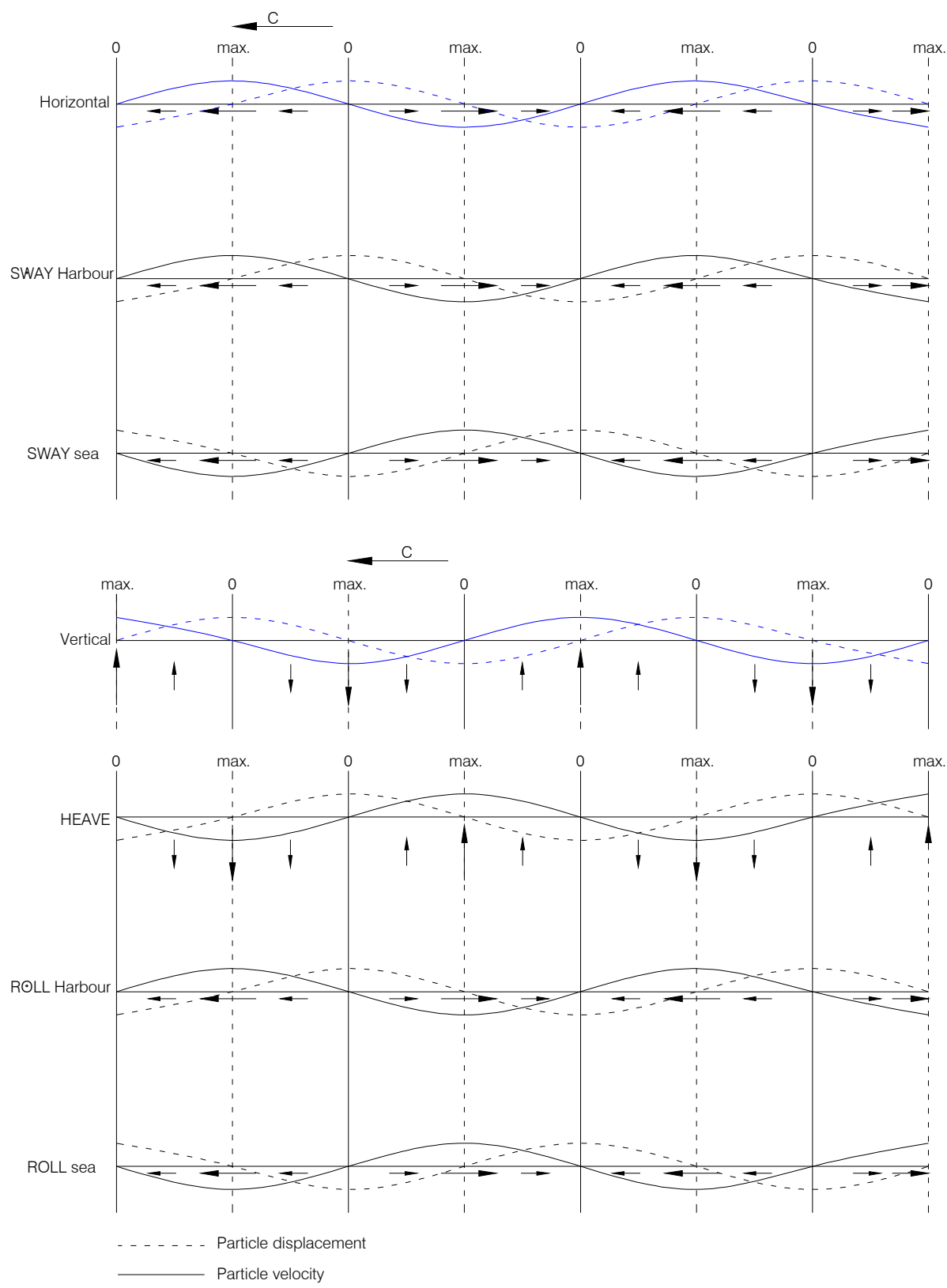

Figure B-20: Wave properties, produced by the floating body reaction on incoming waves. 
The equations of the heave, sway and roll motions and the equations of the waves that are produced by these motions are given in table B-12.

\begin{tabular}{|c|c|c|}
\hline & Wave particle property & \\
\hline \multirow{3}{*}{ Incoming wave } & Displacement & $\zeta_{w v}=\zeta_{a} \cdot \sin (\omega t)$ \\
\hline & Velocity & $\dot{\zeta}_{w v}=\zeta_{a} \cdot \omega \cos (\omega t)$ \\
\hline & Acceleration & $\ddot{\zeta}_{w v}=-\zeta_{a} \cdot \omega^{2} \sin (\omega t)$ \\
\hline \multirow{3}{*}{ SWAY motion } & Displacement & $y=-y_{a} \cdot \cos (\omega t)$ \\
\hline & Velocity & $\dot{y}=y_{a} \cdot \omega \sin \left(\omega t+\varepsilon_{y \zeta}\right)$ \\
\hline & Acceleration & $\ddot{y}=y_{a} \cdot \omega^{2} \cos \left(\omega t+\varepsilon_{y \zeta}\right)$ \\
\hline \multirow{3}{*}{$\begin{array}{l}\text { SWAY transmission } \\
\text { harbour }\end{array}$} & Displacement & $\zeta_{T ; \text { sway }}=-\vartheta_{y_{a}} \cdot \cos \left(\omega t+\varepsilon_{y \zeta}\right)$ \\
\hline & Velocity & $\dot{\zeta}_{T ; s w a y}=\vartheta_{y_{a}} \cdot \omega \sin \left(\omega t+\varepsilon_{y \zeta}\right)$ \\
\hline & Acceleration & $\ddot{\zeta}_{T ; \text { sway }}=\vartheta_{y_{a}} \cdot \omega^{2} \cos \left(\omega t+\varepsilon_{y \zeta}\right)$ \\
\hline \multirow{3}{*}{$\begin{array}{l}\text { SWAY reflection } \\
\text { sea }\end{array}$} & Displacement & $\zeta_{R ; \text { sway }}=\vartheta_{y_{a}} \cdot \cos \left(\omega t+\varepsilon_{y \zeta}\right)$ \\
\hline & Velocity & $\dot{\zeta}_{R ; s w a y}=-\vartheta_{y_{a}} \cdot \omega \sin \left(\omega t+\varepsilon_{y \zeta}\right)$ \\
\hline & Acceleration & $\ddot{\zeta}_{R ; \text { sway }}=-\vartheta_{y_{a}} \cdot \omega^{2} \cos \left(\omega t+\varepsilon_{y \zeta}\right)$ \\
\hline \multirow{3}{*}{ HEAVE motion } & Displacement & $z=z_{a} \cdot \sin \left(\omega t+\varepsilon_{z \zeta}\right)$ \\
\hline & Velocity & $\dot{z}=z_{a} \cdot \omega \cos \left(\omega t+\varepsilon_{z \zeta}\right)$ \\
\hline & Acceleration & $\ddot{z}=-z_{a} \cdot \omega^{2} \sin \left(\omega t+\varepsilon_{z \zeta}\right)$ \\
\hline \multirow{3}{*}{$\begin{array}{l}\text { HEAVE } \\
\text { transmission } \\
\text { Harbour and sea }\end{array}$} & Displacement & $\zeta_{T ; \text { heave }}=-\vartheta_{z_{a}} \cdot \cos \left(\omega t+\varepsilon_{z \zeta}\right)$ \\
\hline & Velocity & $\dot{\zeta}_{T ; \text { heave }}=\vartheta_{z_{a}} \cdot \omega \sin \left(\omega t+\varepsilon_{z \zeta}\right)$ \\
\hline & Acceleration & $\ddot{\zeta}_{T ; \text { heave }}=\vartheta_{z_{a}} \cdot \omega^{2} \cos \left(\omega t+\varepsilon_{z \zeta}\right)$ \\
\hline \multirow{3}{*}{ ROLL motion } & Displacement & $\phi=-\phi_{a} \cdot \cos (\omega t)$ \\
\hline & Velocity & $\dot{\phi}=\phi_{a} \cdot \omega \sin \left(\omega t+\varepsilon_{\phi \zeta}\right)$ \\
\hline & Acceleration & $\ddot{\phi}=\phi_{a} \cdot \omega^{2} \cos \left(\omega t+\varepsilon_{\phi \zeta}\right)$ \\
\hline \multirow{3}{*}{$\begin{array}{l}\text { ROLL transmission } \\
\text { harbour }\end{array}$} & Displacement & $\zeta_{T ; \text { roll }}=-\vartheta_{\phi_{a}} \cdot \cos \left(\omega t+\varepsilon_{\phi \zeta}\right)$ \\
\hline & Velocity & $\dot{\zeta}_{T ; \text { roll }}=\vartheta_{\phi_{a}} \cdot \omega \sin \left(\omega t+\varepsilon_{\phi \zeta}\right)$ \\
\hline & Acceleration & $\ddot{\zeta}_{T ; \text { roll }}=\vartheta_{\phi_{a}} \cdot \omega^{2} \cos \left(\omega t+\varepsilon_{\phi \zeta}\right)$ \\
\hline \multirow{3}{*}{ ROLL reflection sea } & Displacement & $\zeta_{T ; \text { roll }}=\vartheta_{\phi_{a}} \cdot \cos \left(\omega t+\varepsilon_{\phi \zeta}\right)$ \\
\hline & Velocity & $\dot{\zeta}_{T ; \text { roll }}=-\vartheta_{\phi_{a}} \cdot \omega \sin \left(\omega t+\varepsilon_{\phi \zeta}\right)$ \\
\hline & Acceleration & $\ddot{\zeta}_{T ; \text { roll }}=-\vartheta_{\phi_{a}} \cdot \omega^{2} \cos \left(\omega t+\varepsilon_{\phi \zeta}\right)$ \\
\hline
\end{tabular}

Table B-12: Two-dimensional equations of motion

Where:

$\vartheta_{n_{a}} \quad: \quad$ Motion related wave amplitude 


\section{B.V.a The phase shift}

The phase shift is a very important factor in creating an anti-wave. The anti-wave should be large enough to sufficiently attenuate the wave that has transmitted due to the underflow. Possible situations that can occur are shown in the figure below. The wave amplitudes, as they are presented in figure B-21, are randomly chosen and are only used to find the point where the maximum of the total wave occurs.

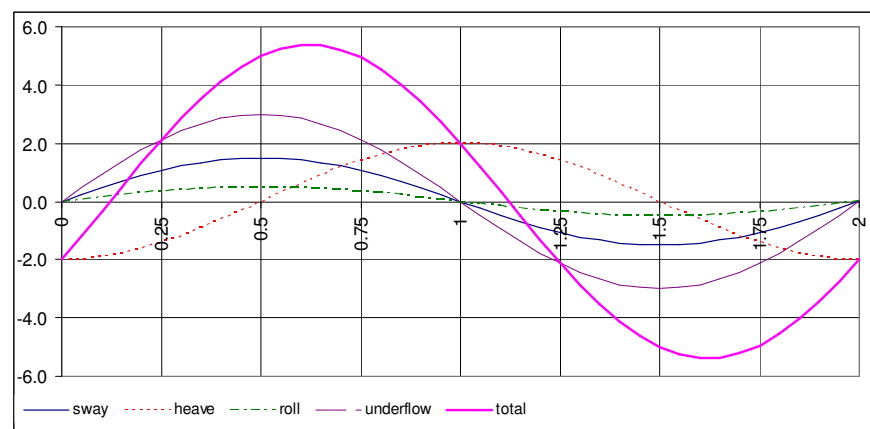

(a)

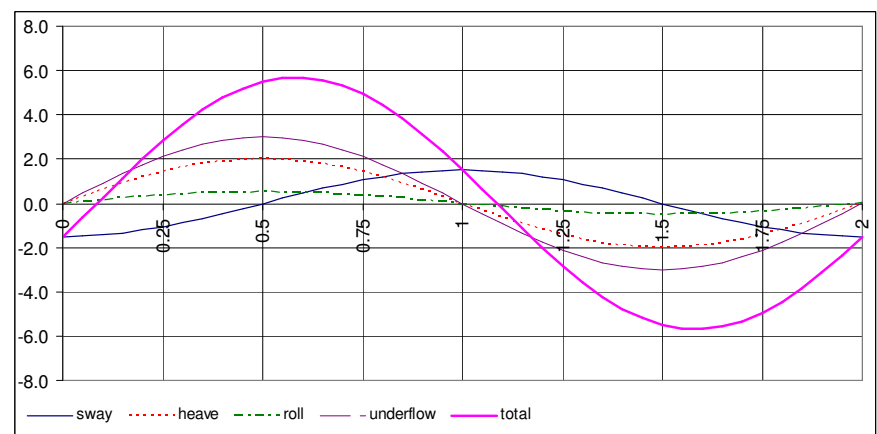

(c)

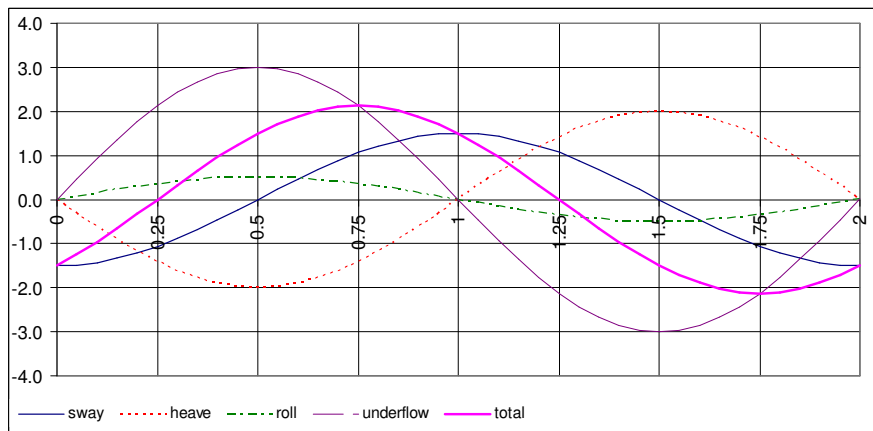

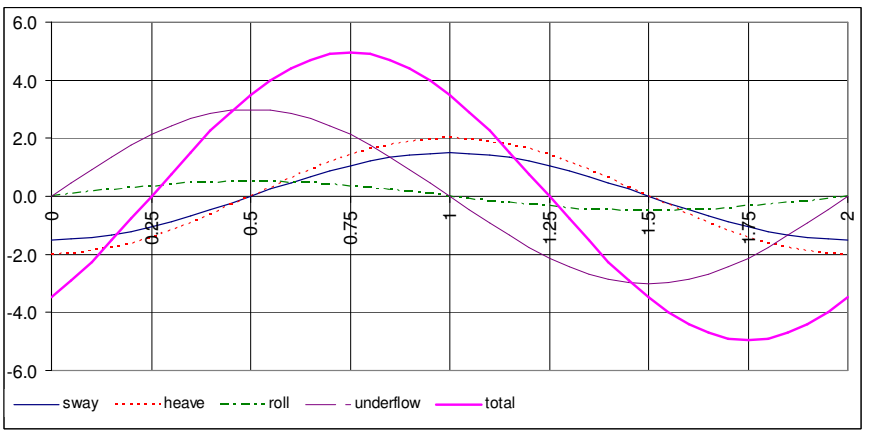

(b)

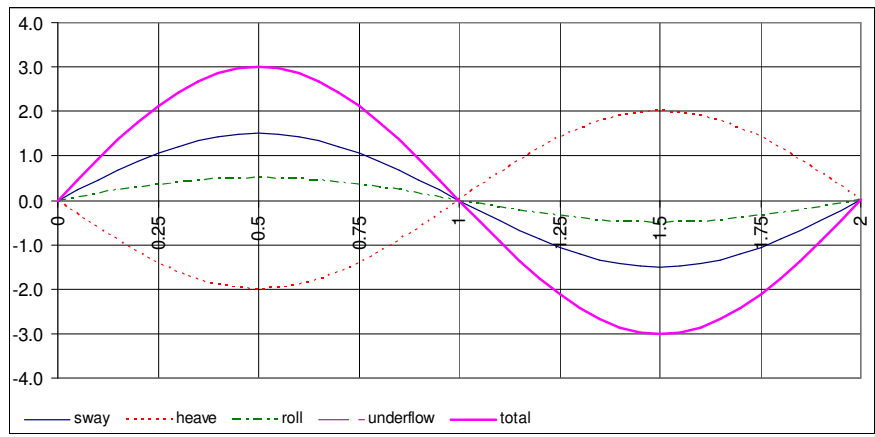

(d)

Figure B-21: Phase shift influence. (a): $\varepsilon_{y \zeta}=0, \varepsilon_{z \zeta}=0 \mid$ (b): $\varepsilon_{y \zeta}=-1 / 2 \pi, \quad \varepsilon_{z \zeta}=0 \mid$ (c): $\varepsilon_{y \zeta}=-1 / 2 \pi, \varepsilon_{z \zeta}=1 / 2 \pi$ | (d) $\varepsilon_{y \zeta}=0, \varepsilon_{z \zeta}=-1 / 2 \pi \quad$ (e) $\varepsilon_{y \zeta}=-1 / 2 \pi, \varepsilon_{z \zeta}=-1 / 2 \pi$

The amplitudes, as they are presented in figure B-21 are randomly chosen. Although this does not represent reality, it can be concluded that situation (e) gives the best results. The heave motion is the factor that creates the anti-wave in this case. When the amplitude of the wave that is created by the heave motion increases, situation (d) might become preferable.

$\left(\varepsilon_{z \zeta} \geq 0\right)$

$$
: \varepsilon_{\text {tot } ; \text { max }}=\frac{\left\{\zeta_{T ; \text { sway }} \cdot\left(\varepsilon_{y ; 0}-\varepsilon_{y \zeta}\right)+\zeta_{T ; \text { heave }} \cdot\left(\varepsilon_{z ; 0}-\varepsilon_{z \zeta}\right)+\zeta_{T ; \text { roll }} \cdot\left(\varepsilon_{\phi ; 0}-\varepsilon_{\phi \zeta}\right)+\zeta_{U} \cdot\left(\varepsilon_{u ; 0}\right)\right\}}{\left\{\zeta_{T ; \text { sway }}+\zeta_{T ; \text { heave }}+\zeta_{T ; \text { roll }}+\zeta_{U}\right\}}
$$$$
\left.\left(\varepsilon_{z \zeta}=-1 / 2 \pi, \varepsilon_{y \zeta}=-1 / 2 \pi\right): \varepsilon_{\text {tot } ; \max }=\frac{\left\{\zeta_{T ; \text { sway }} \cdot\left(\varepsilon_{y ; 0}+\varepsilon_{y \zeta}\right)+\zeta_{T ; \text { heave }} \cdot\left(\varepsilon_{z ; 0}-\varepsilon_{z \zeta}\right)+\zeta_{T ; \text { roll }} \cdot\left(\varepsilon_{\phi ; 0}-\varepsilon_{\phi \zeta}\right)+\zeta_{U} \cdot\left(\varepsilon_{u ; 0}\right)\right\}}{\left\{\zeta_{T ; \text { sway }}+\zeta_{T ; \text { heave }}+\zeta_{T ; \text { roll }}+\zeta_{U}\right\}}\right\}(B-27
$$$$
\left(\varepsilon_{z \zeta}=-1 / 2 \pi\right)
$$

$$
\left.: \varepsilon_{\text {tot } ; \max }=\frac{\left\{\zeta_{T ; \text { sway }} \cdot\left(\varepsilon_{y ; 0}+\varepsilon_{y \zeta}\right)+\zeta_{T ; \text { heave }} \cdot\left(\varepsilon_{z ; 0}+\varepsilon_{z \zeta}\right)+\zeta_{T ; \text { roll }} \cdot\left(\varepsilon_{\phi ; 0}-\varepsilon_{\phi \zeta}\right)+\zeta_{U} \cdot\left(\varepsilon_{u ; 0}\right)\right\}}{\left\{\zeta_{T ; \text { sway }}+\zeta_{T ; \text { heave }}+\zeta_{T ; \text { roll }}+\zeta_{U}\right\}}\right\}
$$




\section{B.V.b The Natural frequency}

Heave natural frequency, or undamped resonance frequency:

$\omega_{n 3}=\sqrt{\left(\frac{\rho g \cdot A_{s}}{m+a_{33}}\right)}$

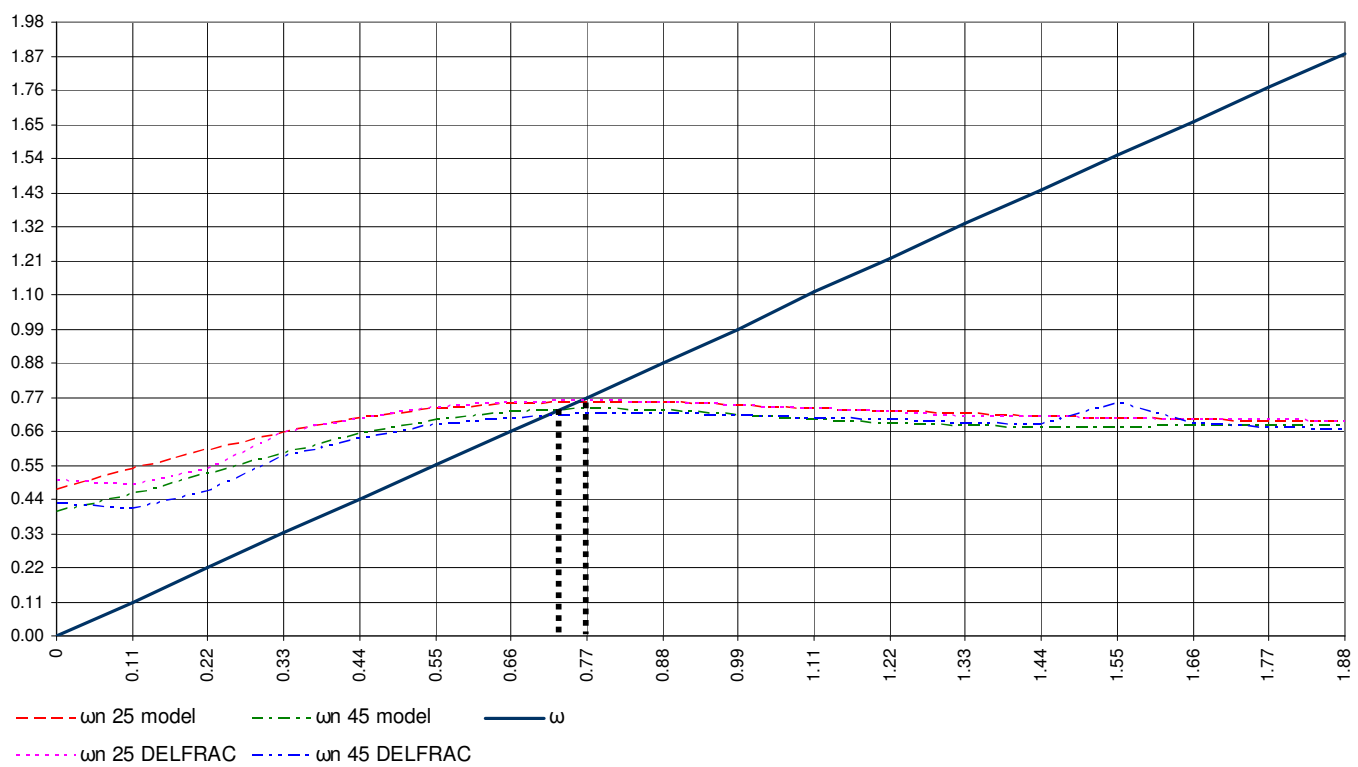

Figure B-22

Roll natural frequency:

$\omega_{n 4}=\sqrt{\left(\frac{\rho g \cdot A_{w} \cdot \overline{G M}_{T}}{m \cdot k_{44}^{2}+a_{44}}\right)}$

$k_{44}=0.35 \cdot Y_{w}$

$A_{w} \quad: \quad$ displaced volume of water.

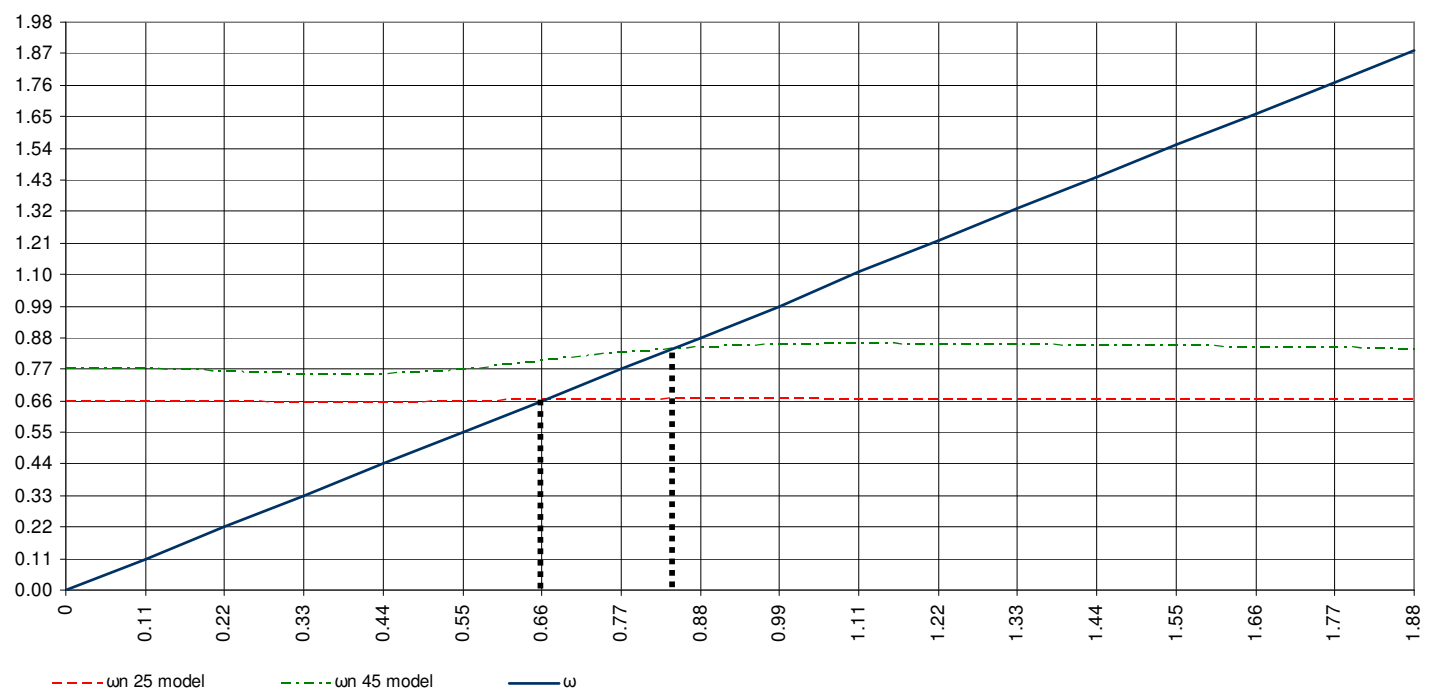

Figure B-23: 


\section{B.V.c Sway}

The sway motion of the floating breakwater in a 2-dimensional plane is described as stated below:

$$
\left(\rho \nabla+a_{22}\right) \cdot \ddot{y}+\left(d_{b y}+b_{22}\right) \cdot \dot{y}+\left(k_{c y}+c_{22}\right) \cdot y=a_{22} \cdot \ddot{\zeta}_{w 2}^{*}+b_{22} \cdot \dot{\zeta}_{w 2}^{*}+c_{22} \cdot \zeta_{w 2}^{*}
$$

Since the primary design calculation will be performed with regular, straight incoming waves, the influence of yawing on the sway motion is negligible.

\section{Calculation Parameters}

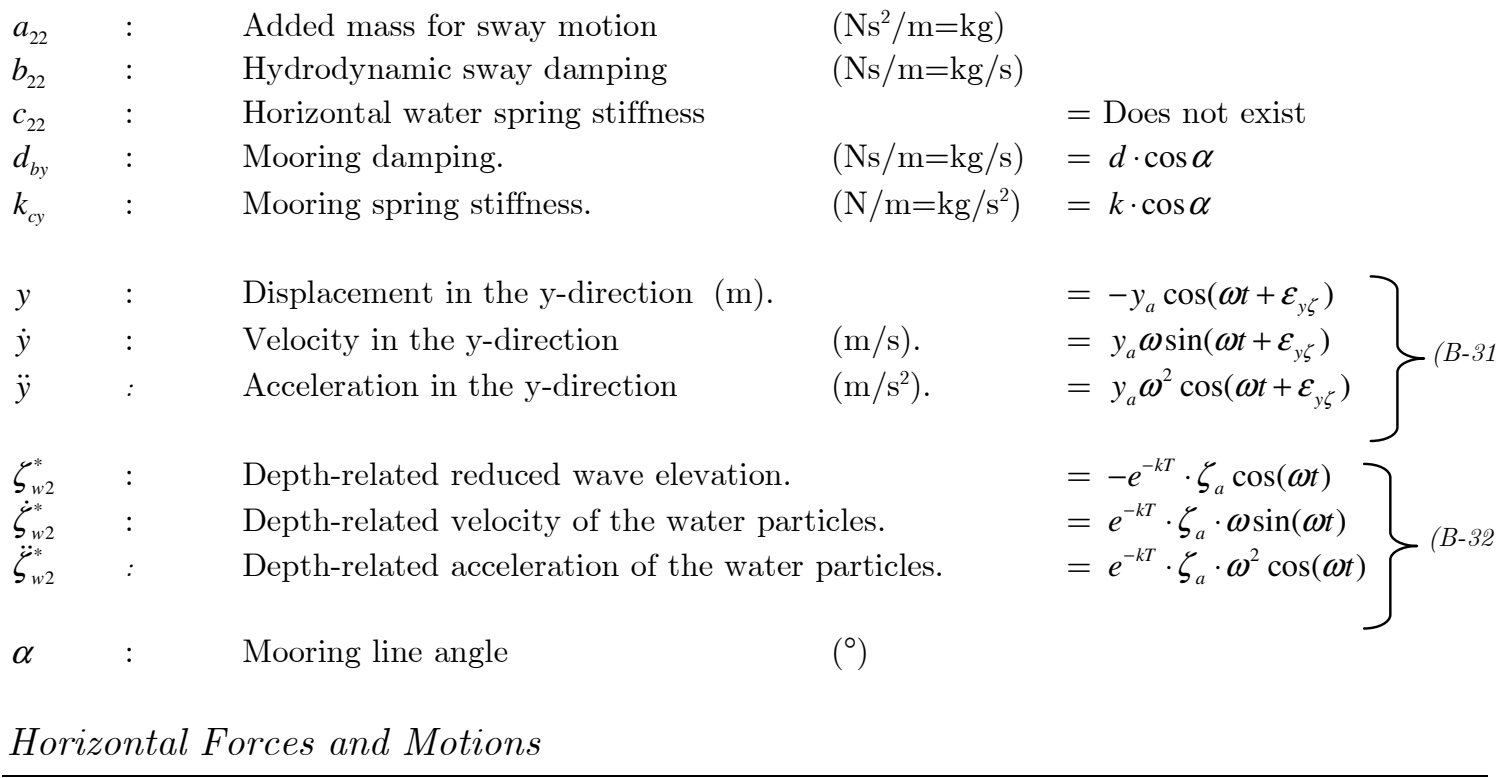

\section{Horizontal wave force}

$F_{w}=a_{22} \cdot \ddot{\zeta}_{w 2}+b_{22} \cdot \dot{\zeta}_{w 2}$

A substitution of B.32 in B.33 yields:

$$
F_{w}=\zeta_{a} \cdot e^{-k T_{2}}\left\{a_{22} \omega^{2}\right\} \cos (\omega t)+\zeta_{a} \cdot e^{-k T_{2}}\left\{b_{22} \omega\right\} \sin (\omega t)
$$

The horizontal wave force can be separated independently in terms of in-phase and out-of phase terms:

$$
F_{w}=F_{a} \cos \left(\omega t+\varepsilon_{F \zeta}\right)=F_{a} \cos \left(\varepsilon_{F \zeta}\right) \cos (\omega t)+F_{a} \sin \left(\varepsilon_{F \zeta}\right) \sin (\omega t)
$$

Combining the two in-phase and out-of phase terms of equations B.39 and B.35 yields:

$$
\begin{aligned}
& F_{a} \cos \left(\varepsilon_{F \zeta}\right)=\zeta_{a} \cdot e^{-k T_{2}}\left\{a_{22} \omega^{2}\right\} \\
& F_{a} \sin \left(\varepsilon_{F \zeta}\right)=\zeta_{a} \cdot e^{-k T_{2}}\left\{b_{22} \omega\right\}
\end{aligned}
$$

Adding the squares of these two equations results in the horizontal wave force amplitude at a depth ' $\mathrm{T}$ ' on the construction:

$$
F_{a}=\zeta_{a} \cdot e^{-k T_{2}} \sqrt{\left\{a_{22} \omega^{2}\right\}^{2}+\left\{b_{22} \omega\right\}^{2}}
$$




\section{Sway motion}

A substitution of B.32 and B.34 into the equation of sway motion of B.30 yields:

$$
\left(m+a_{22}\right) \cdot y_{a} \omega^{2} \cos \left(\omega t+\varepsilon_{y \zeta}\right)+\left(d+b_{22}\right) \cdot y_{a} \omega \sin \left(\omega t+\varepsilon_{y \zeta}\right)-k \cdot y_{a} \cos \left(\omega t+\varepsilon_{y \zeta}\right)=F_{w}
$$

After ordering the factors on sinus and cosinus, this equation becomes:

$$
\begin{aligned}
& y_{a}\left\{-k+\left(m+a_{22}\right) \omega^{2}\right\} \cos \left(\omega t+\varepsilon_{y \zeta}\right)+y_{a}\left\{\left(d+b_{22}\right) \omega\right\} \sin \left(\omega t+\varepsilon_{y \zeta}\right)= \\
& \zeta_{a} \cdot e^{-k T_{2}}\left\{a_{22} \omega^{2}\right\} \cos (\omega t)+\zeta_{a} \cdot e^{-k T_{2}}\left\{b_{22} \omega\right\} \sin (\omega t)
\end{aligned}
$$

Splitting the angle $\left(\omega t+\varepsilon_{y \zeta}\right)$ and writing the in-phase term and the out-of phase term separately:

$$
\begin{aligned}
& y_{a}\left\{\left\{-k+\left(m+a_{22}\right) \omega^{2}\right\} \cos \left(\varepsilon_{y \zeta}\right)+\left\{\left(d+b_{22}\right) \omega\right\} \sin \left(\varepsilon_{y \zeta}\right)\right\} \cdot \cos (\omega t)+ \\
& y_{a}\left\{\left\{-k+\left(m+a_{22}\right) \omega^{2}\right\} \sin \left(\varepsilon_{y \zeta}\right)+\left\{\left(d+b_{22}\right) \omega\right\} \cos \left(\varepsilon_{y \zeta}\right)\right\} \cdot \sin (\omega t)= \\
& \zeta_{a} \cdot e^{-k T_{2}}\left\{a_{22} \omega^{2}\right\} \cos (\omega t)+\zeta_{a} \cdot e^{-k T_{2}}\left\{b_{22} \omega\right\} \sin (\omega t)
\end{aligned}
$$

By equating the two out-of-phase terms and the two in-phase terms, one obtains two equations with two unknowns:

$$
\begin{aligned}
& y_{a}\left\{\left\{-k+\left(m+a_{22}\right) \omega^{2}\right\} \cos \left(\varepsilon_{y \zeta}\right)+\left\{\left(d+b_{22}\right) \omega\right\} \sin \left(\varepsilon_{y \zeta}\right)\right\}=\zeta_{a} \cdot e^{-k T_{2}}\left\{a_{22} \omega^{2}\right\} \\
& y_{a}\left\{\left\{-k+\left(m+a_{22}\right) \omega^{2}\right\} \sin \left(\varepsilon_{y \zeta}\right)+\left\{\left(d+b_{22}\right) \omega\right\} \cos \left(\varepsilon_{y \zeta}\right)\right\}=\zeta_{a} \cdot e^{-k T_{2}}\left\{b_{22} \omega\right\}
\end{aligned}
$$

Adding the squares of these two equations results in the sway amplitude:

$$
\frac{y_{a}}{\zeta_{a}}=e^{-k T_{2}} \sqrt{\frac{\left\{a_{22} \omega^{2}\right\}^{2}+\left\{b_{22} \omega\right\}^{2}}{\left\{-k+\left(m+a_{22}\right) \omega^{2}\right\}^{2}+\left\{\left(d+b_{22}\right) \omega\right\}^{2}}}
$$

The sway motion amplitude for several wave frequencies is shown in figure B-4. When the sway amplitude is compared with the DELFRAC calculation, a large difference is visible. This difference is due to the drag force that is not involved in this calculation model, as clarified in chapter 6.4.1.

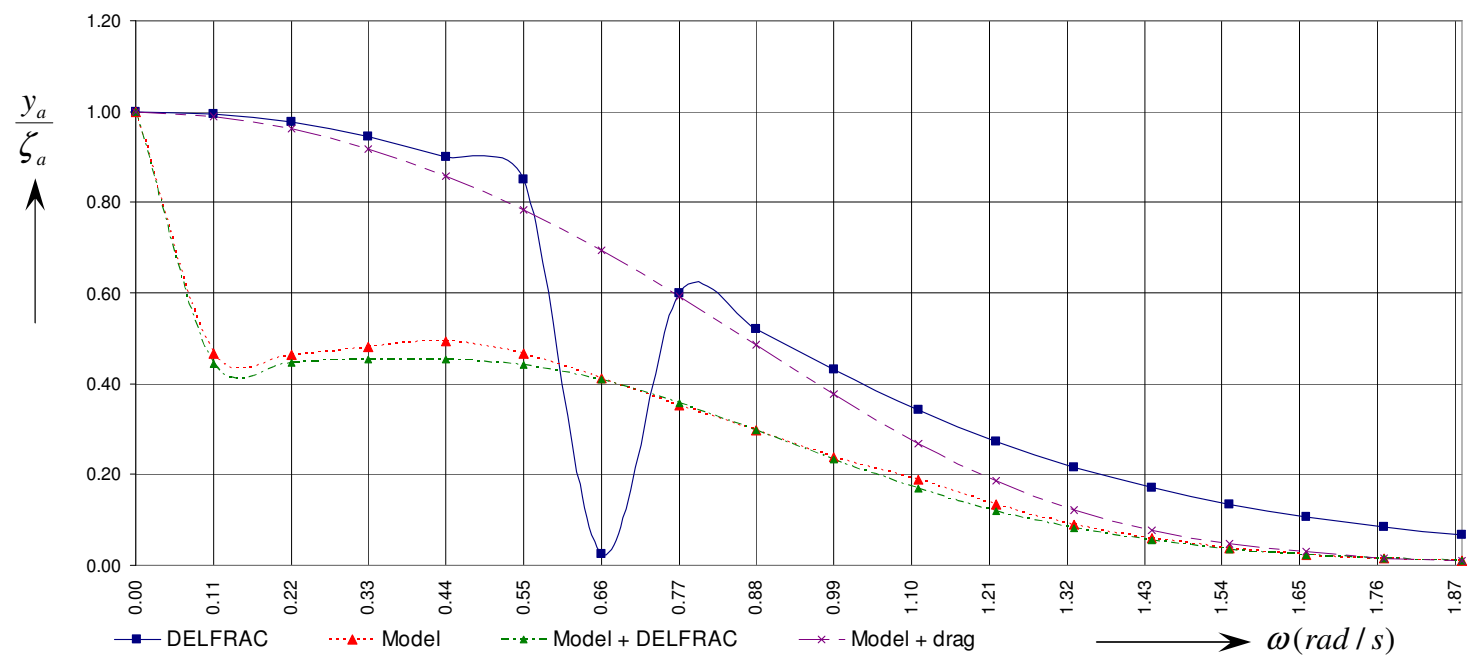

Figure B-24: Sway reaction at several wave periods $(m=200,000 \mathrm{~kg} / \mathrm{Yw}=25 \mathrm{~m})$. 
Elimination of $y a / \zeta_{a} \cdot e^{-k T_{2}}$ in the equations of B.41 leads to the phase shift:

$\varepsilon_{y \zeta}=\arctan \left\{\frac{m b_{22} \omega^{3}}{\left(a_{22} \omega^{2}\right)\left\{-k+\left(m+a_{22}\right) \omega^{2}+\left\{\left(d+b_{22}\right) \omega\right\}^{2}\right\}}\right\}$

with: $0 \leq \varepsilon_{y \zeta} \leq 2 \pi$

This makes the total sway motion of the system:

$y=-y_{a} \cos \left(\omega t+\varepsilon_{y \zeta}\right)$

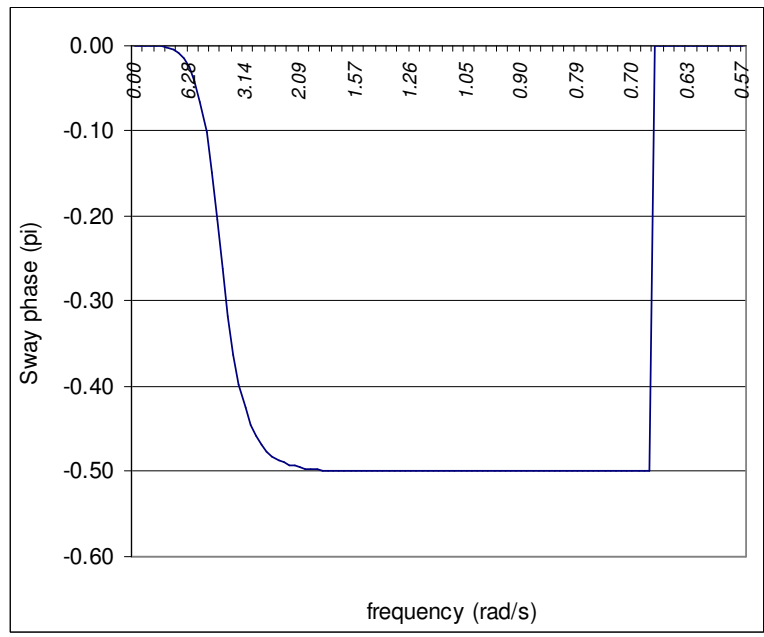




\section{Energy Consideration}

Suppose the breakwater is carrying out a horizontal harmonic oscillation:

$$
y=y_{a} \sin \omega t
$$

To understand the amount of energy the sway motion of the floating breakwater passes on to the still water level on the harbour-side, one has to determine the amount of work done by the mass, damping and spring force components per unit of time during one period of oscillation $\mathrm{T}$.

Mass:

$$
E_{m}=\frac{1}{T} \int_{0}^{T}\left\{\left(m_{22}+a_{22}\right) \cdot \ddot{y}\right\} \cdot\{\dot{y} \cdot d t\}=\frac{-y_{a}^{2}\left(m_{22}+a_{22}\right) \omega^{3}}{T} \int_{0}^{T} \cos \omega t \cdot \sin \omega t \cdot d t=0
$$

Damping:

$$
E_{d}=\frac{1}{T} \int_{0}^{T}\left\{b_{22} \cdot \dot{y}\right\} \cdot\{\dot{y} \cdot d t\}=\frac{y_{a}^{2} b_{22} \omega^{2}}{T} \int_{0}^{T} \cos ^{2} \omega t \cdot d t=\frac{1}{2} b_{22} \omega^{2} y_{a}^{2}
$$

Spring:

$$
\text { Horizontal spring stiffness does not exist in water. }
$$

Only the damping force $(b \cdot \dot{y})$ dissipates energy, in other words: damping is the reason why the sway motion dies out. This damping energy is exactly the amount of energy which is dissipated by the waves on both sides of the structure. The wave energy per floating breakwater section $\left(\mathrm{L}_{\mathrm{w}}\right)$ times the covered distance by the radiated wave energy in one period divided by the time gives:

$$
E=\frac{1}{T} \cdot 2 \cdot L_{w} \cdot\left\{\frac{1}{2} \rho g \zeta_{c}^{2}\right\} \cdot\left\{c_{g} \cdot T\right\}=\frac{L_{w} \cdot \rho g^{2} \zeta_{c}^{2}}{2 \omega}
$$

Where:

$$
\begin{array}{lll}
\frac{1}{2} \rho g \zeta_{c}^{2} & : & \text { Wave energy per unit area } \\
c_{g}=\frac{g}{2 \omega} & : & \text { Group velocity (deep water) }
\end{array}
$$

Equalising equations B.44 and B.45 gives the (vertical) transmitted wave due to the sway motion.

$$
\zeta_{T ; \text { sway }}(t)=\sqrt{\frac{b_{22} \cdot \omega^{3} \cdot y_{a}^{2}}{L_{w} \cdot \rho \cdot g^{2}}} \cdot \sin \left(\omega t+\varepsilon_{y \zeta}\right)
$$

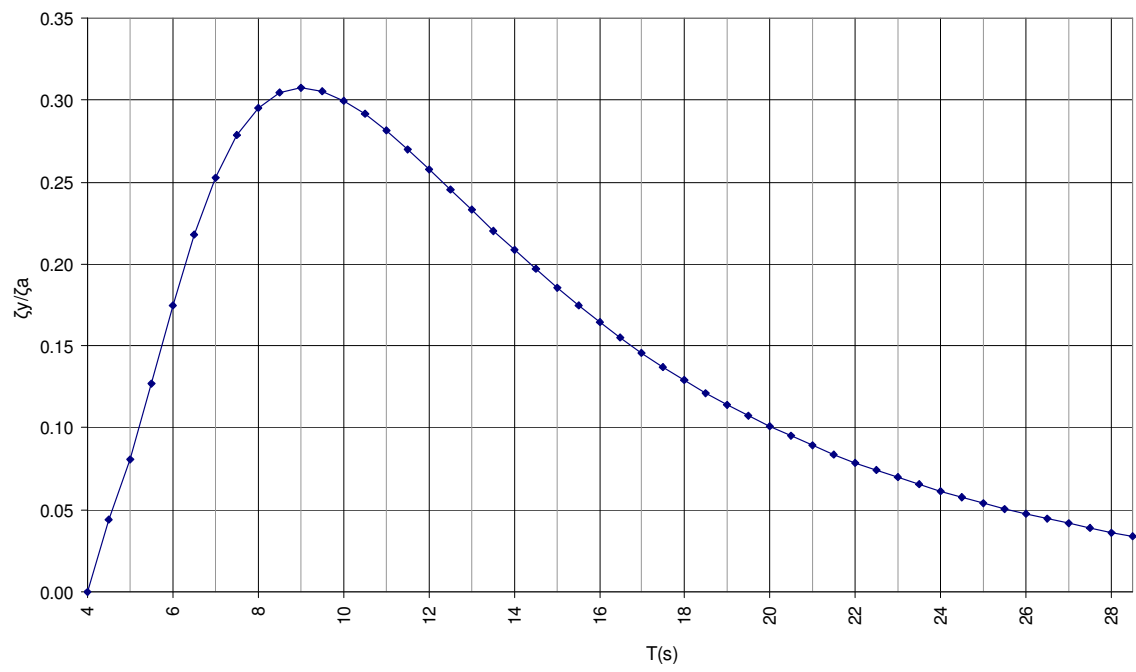

Figure B-25: Wave transmission due to the sway motion $\left(T_{1}=15 \mathrm{~m} / Y_{w}=65 \mathrm{~m}\right)$.

The sway factors in table B-12 become:

$\vartheta_{y_{a}} \quad: \quad$ Amplitude of the wave, produced by the heave motion $=\sqrt{\frac{b_{22} \cdot \omega^{3} \cdot y_{a}^{2}}{L_{w} \cdot \rho \cdot g^{2}}}$ 


\section{B.V.d Heave}

The heave motion of the floating breakwater in a 2-dimensional plane is described as stated below:

$$
\left(\rho \nabla+a_{33}\right) \cdot \ddot{z}+\left(d_{b z}+b_{33}\right) \cdot \dot{z}+\left(k_{c z}+c_{33}\right) \cdot z=a_{33} \cdot \ddot{\zeta}_{w 3}^{*}+b_{33} \cdot \dot{\zeta}_{w 3}^{*}+c_{33} \cdot \zeta_{w 3}^{*} \cdot C_{3}
$$

Since the primary design calculation will be performed with regular, perpendicular incoming waves, the influence of pitch on the 2D-heave motion is negligible.

\section{Calculation Parameters}

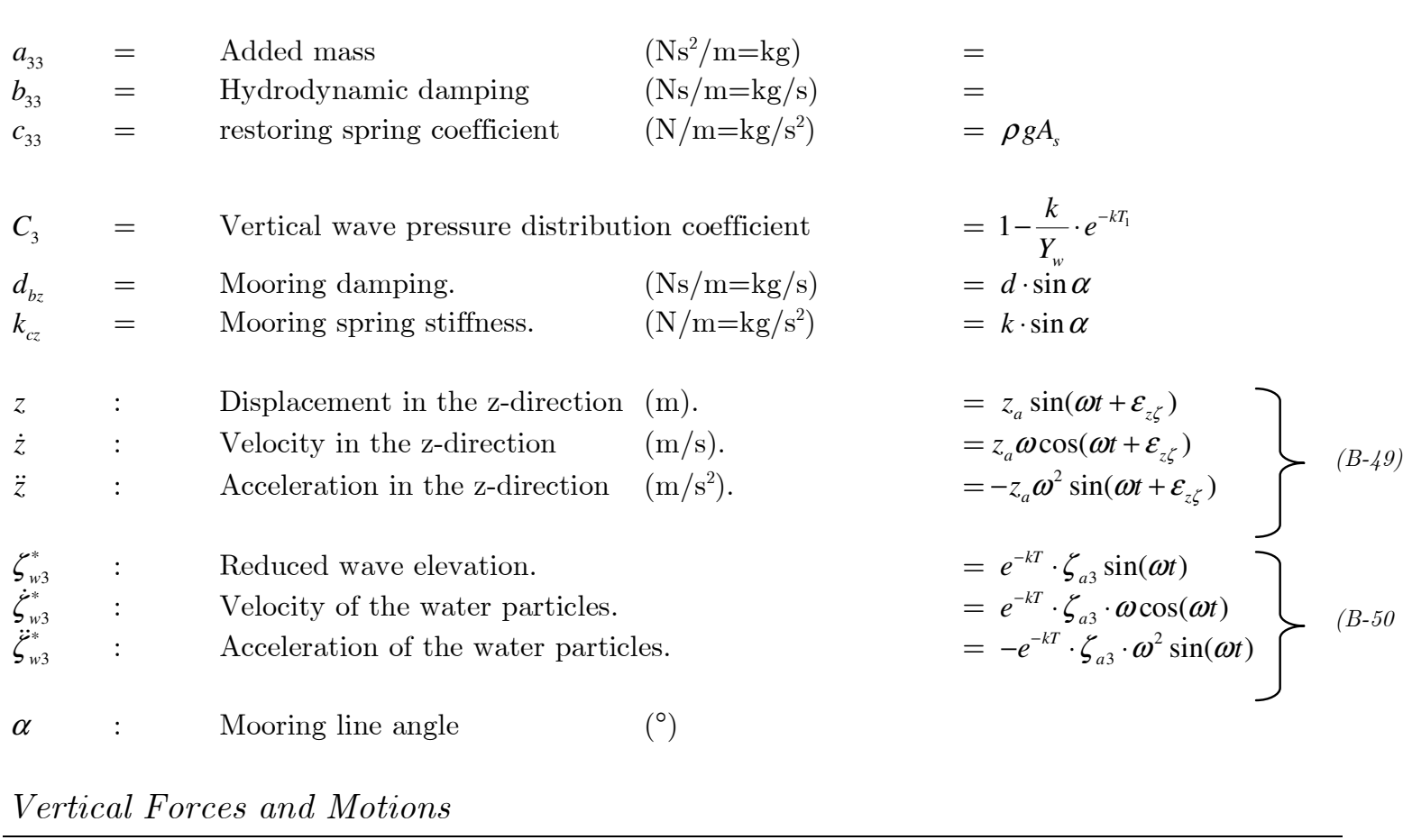

\section{Vertical wave force}

$F_{w}=a_{33} \cdot \ddot{\zeta}_{w 3}^{*}+b_{33} \cdot \dot{\zeta}_{w 3}^{*}+c_{33} \cdot \zeta_{w 3}^{*} \cdot C_{3}$

A substitution of B.50 in B.48 yields:

$$
F_{w}=-\zeta_{a} \cdot e^{-k T_{1}}\left\{c_{33} \cdot C_{3}-a_{33} \omega^{2}\right\} \sin (\omega t)+\zeta_{a} \cdot e^{-k T_{1}}\left\{b_{33} \omega\right\} \cos (\omega t)
$$

The vertical wave force can be separated independently in terms of in-phase and out-of-phase terms:

$$
F_{w}=F_{a} \sin \left(\omega t+\varepsilon_{F \zeta}\right)=-F_{a} \sin \left(\varepsilon_{F \zeta}\right) \sin (\omega t)+F_{a} \cos \left(\varepsilon_{F \zeta}\right) \cos (\omega t)
$$

Combining the two in-phase and out-of-phase terms of equations B.52 and B.53 yields:

$$
\begin{aligned}
& F_{a} \sin \left(\varepsilon_{F \zeta}\right)=\zeta_{a} \cdot e^{-k T_{1}}\left\{c_{33} \cdot C_{3}-a_{33} \omega^{2}\right\} \\
& F_{a} \cos \left(\varepsilon_{F \zeta}\right)=\zeta_{a} \cdot e^{-k T_{1}}\left\{b_{33} \omega\right\}
\end{aligned}
$$

Adding the squares of these two equations results in the vertical wave force amplitude at depth ' $\mathrm{T}_{1}$ ' under the construction:

$$
F_{a}=\zeta_{a} \cdot e^{-k T_{1}} \sqrt{\left\{c_{33} \cdot C_{3}-a_{33} \omega^{2}\right\}^{2}+\left\{b_{33} \omega\right\}^{2}}
$$




\section{Heave motion}

A substitution of B.49 and B.52 into the equation of heave motion of B.37 yields:

$$
-\left(m+a_{33}\right) \cdot z_{a} \omega^{2} \sin \left(\omega t+\varepsilon_{z \zeta}\right)+\left(d+b_{33}\right) \cdot z_{a} \omega \cos \left(\omega t+\varepsilon_{z \zeta}\right)+\left(k+c_{33}\right) \cdot z_{a} \sin \left(\omega t+\varepsilon_{z \zeta}\right)=F_{w}
$$

After ordering the factors on sinus and cosinus, this equation becomes:

$$
\begin{aligned}
& z_{a}\left\{\left(k+c_{33}\right)-\left(m+a_{33}\right) \omega^{2}\right\} \sin \left(\omega t+\varepsilon_{z \zeta}\right)+z_{a}\left\{\left(d+b_{33}\right) \omega\right\} \cos \left(\omega t+\varepsilon_{z \zeta}\right)= \\
& \zeta_{a} \cdot e^{-k T_{1}}\left\{c_{33} \cdot C_{3}-a_{33} \omega^{2}\right\} \sin (\omega t)+\zeta_{a} \cdot e^{-k T_{1}}\left\{b_{33} \omega\right\} \cos (\omega t)
\end{aligned}
$$

Splitting the angle $\left(\omega t+\varepsilon_{z \zeta}\right)$ and writing the in-phase and the out-of-phase terms separately:

$$
\begin{aligned}
& z_{a}\left\{\left\{\left(k+c_{33}\right)-\left(m+a_{33}\right) \omega^{2}\right\} \sin \left(\varepsilon_{z \zeta}\right)+\left\{\left(d+b_{33}\right) \omega\right\} \cos \left(\varepsilon_{z \zeta}\right)\right\} \cdot \sin (\omega t)+ \\
& z_{a}\left\{\left\{\left(k+c_{33}\right)-\left(m+a_{33}\right) \omega^{2}\right\} \cos \left(\varepsilon_{z \zeta}\right)+\left\{\left(d+b_{33}\right) \omega\right\} \sin \left(\varepsilon_{z \zeta}\right)\right\} \cdot \cos (\omega t)= \\
& \zeta_{a} \cdot e^{-k T_{1}}\left\{c_{33} \cdot C_{3}-a_{33} \omega^{2}\right\} \sin (\omega t)+\zeta_{a} \cdot e^{-k T_{1}}\left\{b_{33} \omega\right\} \cos (\omega t)
\end{aligned}
$$

By equating the two out-of-phase terms and the two in-phase terms, one obtains two equations with two unknowns:

$$
\begin{aligned}
& z_{a}\left\{\left\{\left(k+c_{33}\right)-\left(m+a_{33}\right) \omega^{2}\right\} \sin \left(\varepsilon_{z \zeta}\right)+\left\{\left(d+b_{33}\right) \omega\right\} \cos \left(\varepsilon_{z \zeta}\right)\right\}=\zeta_{a} \cdot e^{-k T_{1}}\left\{c_{33} \cdot C_{3}-a_{33} \omega^{2}\right\} \\
& z_{a}\left\{\left\{\left(k+c_{33}\right)-\left(m+a_{33}\right) \omega^{2}\right\} \cos \left(\varepsilon_{z \zeta}\right)+\left\{\left(d+b_{33}\right) \omega\right\} \sin \left(\varepsilon_{z \zeta}\right)\right\}=\zeta_{a} \cdot e^{-k T_{1}}\left\{b_{33} \omega\right\}
\end{aligned}
$$

Adding the squares of these two equations results in the heave amplitude:

$$
\frac{z_{a}}{\zeta_{a}}=e^{-k T_{1}} \sqrt{\frac{\left\{c_{33} \cdot C_{3}-a_{33} \omega^{2}\right\}^{2}+\left\{b_{33} \omega\right\}^{2}}{\left\{\left(k+c_{33}\right)-\left(m+a_{33}\right) \omega^{2}\right\}^{2}+\left\{\left(d+b_{33}\right) \omega\right\}^{2}}}
$$

The heave motion amplitude for several wave frequencies is shown in figure B-26. When the heave amplitude is compared with the DELFRAC calculation, some difference is visible. The dotted line reflects the difference between the own frequency and the wave frequency. When this parameter is around 0 , the structure will show a larger motion amplitude due to resonance. As we can see in figure B-26, this theory holds for the numerical model, as it is used in this research. The DELFRAC calculation shows a large deviation.

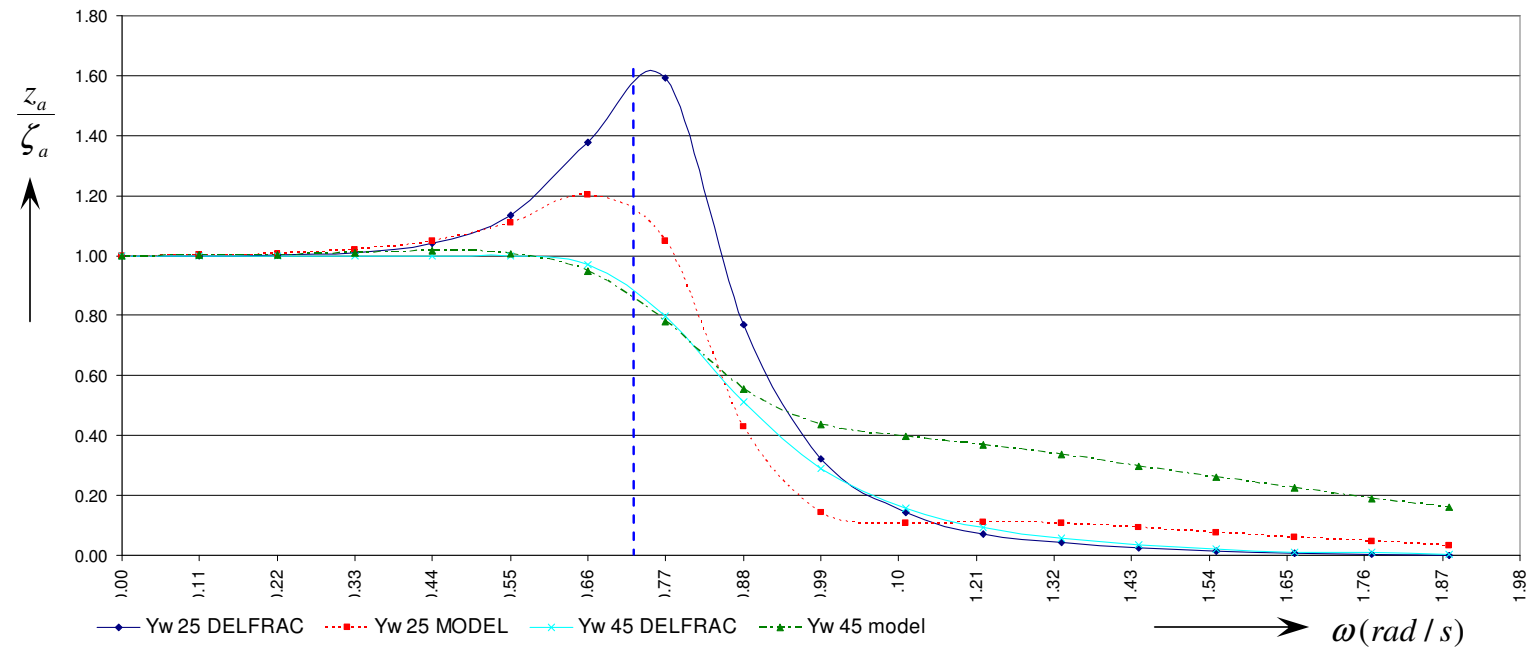

Figure B-26: Heave reaction at several wave frequencies $\left(T_{1}=7.8 \mathrm{~m} / Y_{w}=25 \mathrm{~m}\right)$. 
Elimination of $z_{a} / \zeta_{a} \cdot e^{-k T_{1}}$ in the equations of B.59 leads to the phase shift:

$\varepsilon_{z \zeta}=\arctan \left\{\frac{-m b_{33} \omega^{3}}{\left(c_{33} \cdot C_{3}-a_{33} \omega^{2}\right)\left\{\left(k+c_{33}\right)-\left(m+a_{33}\right) \omega^{2}+\left\{\left(d+b_{33}\right) \omega\right\}^{2}\right\}}\right\} \quad$ with: $0 \leq \varepsilon_{z \zeta} \leq 2 \pi \quad$ (B-61)

This makes the total heave motion of the system:

$z=z_{a} \sin \left(\omega t+\varepsilon_{z \zeta}\right)$

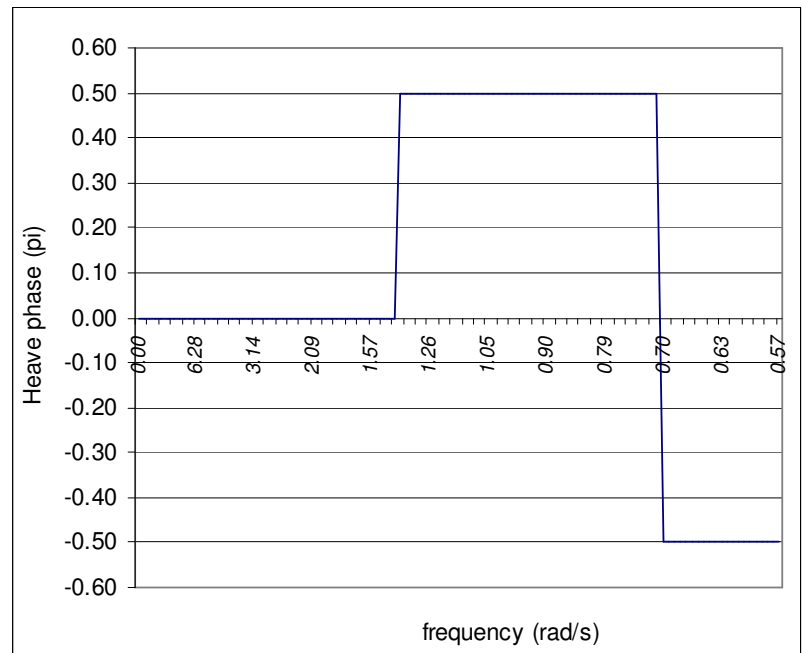




\section{Energy Consideration}

Suppose the breakwater is carrying out a vertical harmonic oscillation:

$z=z_{a} \sin \omega t$

To understand the amount of energy the heave motion of the floating breakwater passes on to the still water level on the harbour-side, one has to determine the amount of work done by the mass, damping and spring force components per unit of time during one period of oscillation $\mathrm{T}$.

Mass:

$$
E_{m}=\frac{1}{T} \int_{0}^{T}\left\{\left(m+a_{33}\right) \cdot \ddot{z}\right\} \cdot\{\dot{z} \cdot d t\}=\frac{-z_{a}^{2}\left(m+a_{33}\right) \omega^{3}}{T} \int_{0}^{T} \sin \omega t \cdot \cos \omega t \cdot d t=0
$$

Damping:

$$
E_{d}=\frac{1}{T} \int_{0}^{T}\left\{b_{33} \cdot \dot{z}\right\} \cdot\{\dot{z} \cdot d t\}=\frac{z_{a}^{2} b_{33} \omega^{2}}{T} \int_{0}^{T} \cos ^{2} \omega t \cdot d t=\frac{1}{2} b_{33} \omega^{2} z_{a}^{2}
$$

Spring:

$$
E_{s}=\frac{1}{T} \int_{0}^{T}\left\{c_{33} \cdot z\right\} \cdot\{\dot{z} \cdot d t\}=\frac{z_{a} c_{33} \omega}{T} \int_{0}^{T} \sin \omega t \cdot \cos \omega t \cdot d t=0
$$

Only the damping force $(b \cdot \dot{z})$ dissipates energy, in other words: damping is the reason why the heave motion dies out. This damping energy is exactly the amount of energy which is dissipated by the waves on both sides of the structure. The wave energy per unit area times the covered distance by the radiated wave energy in one period divided by the time gives:

$$
E=\frac{1}{T} \cdot 2 \cdot L_{w} \cdot\left\{\frac{1}{2} \rho g \zeta_{c}^{2}\right\} \cdot\left\{c_{g} \cdot T\right\}=\frac{L_{w} \cdot \rho g^{2} \zeta_{c}^{2}}{2 \omega}
$$

Equalising equations B.62 and B.63 gives the amplitude of the radiated wave height:

$$
\zeta_{T ; \text { heave }}(t)=\sqrt{\frac{b_{33} \cdot \omega^{3} \cdot z_{a}^{2}}{L_{w} \cdot \rho \cdot g^{2}}} \cos \left(\omega t+\varepsilon_{z \zeta}\right)
$$

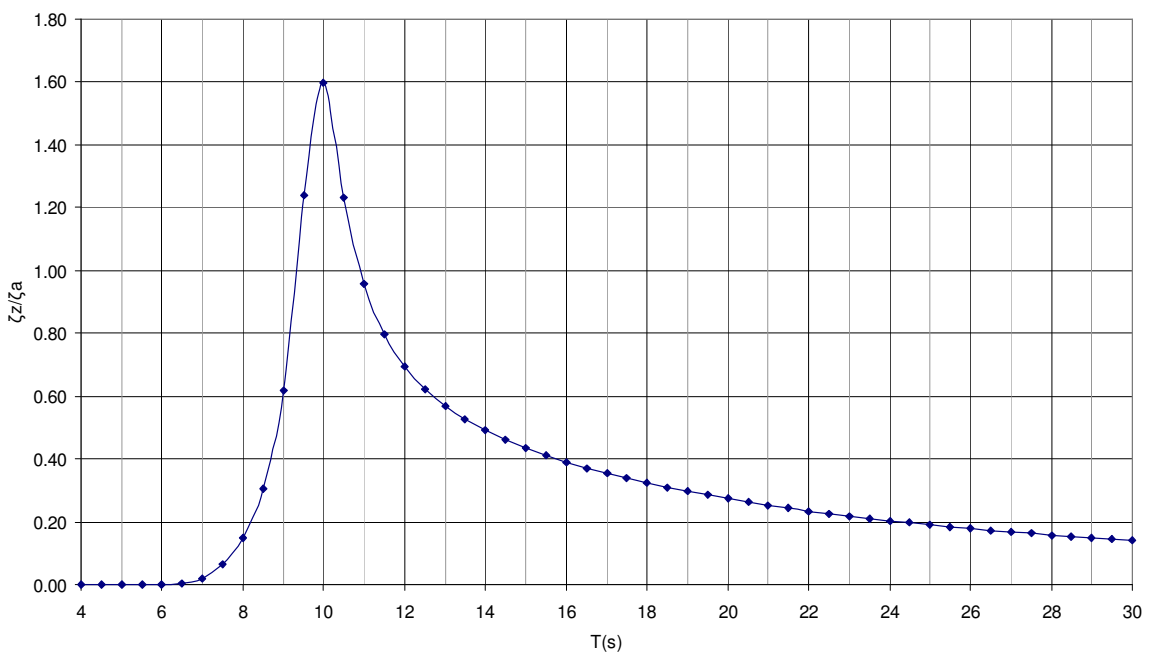

Figure B-27: Wave transmission due to the heave motion $\left(T_{1}=15 \mathrm{~m} / Y_{w}=65 \mathrm{~m}\right)$.

The heave factors in table B-12 become:

$$
\vartheta_{z_{a}} \quad: \quad \text { Amplitude of the wave, produced by the heave motion } \quad=\sqrt{\frac{b_{33} \cdot \omega^{3} \cdot z_{a}^{2}}{L_{w} \cdot \rho \cdot g^{2}}}
$$




\section{B.V.e Underflow}

The total average incoming wave energy per area is denoted as:

$1 / 2 \rho g \zeta_{a}^{2}$

$(B-66)$

The part of the wave energy that is present in the dept range $\left(T_{2} \rightarrow \infty\right)$ is assumed to penetrate for $100 \%$ to the harbour side of the floating breakwater.

This energy will create a transmission wave on the harbour side of the floating breakwater. The energy of this transmitted wave is:

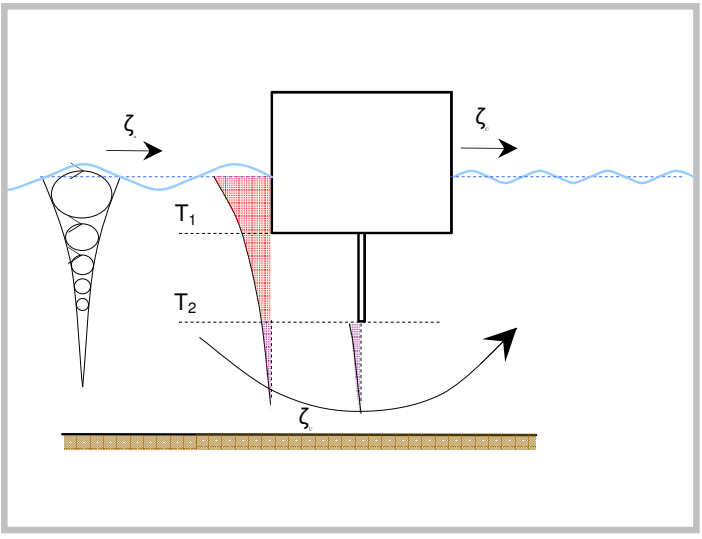

Figure B-28: Energy transmission due to underflow

$1 / 2 \rho g \zeta_{T}^{2}$

B-67)

The energy balance $\mathrm{E}_{\text {underflow }}=\mathrm{E}_{\text {Transmission }}$ is:

$e^{-k\left(T_{2}-z_{a}\right)} \cdot 1 / 2 \rho g \zeta_{a}^{2}=1 / 2 \rho g \zeta_{T}^{2}$

The transmitted wave height becomes:

$\zeta_{T ; U}=\sqrt{e^{-k\left(T_{2}-z_{a}\right)} \cdot \zeta_{a}^{2}}$

The underflow is mainly affected by the structural draft. The heave motion has a certain influence on the underflow when structures with a relative small draft are considered. 


\section{B.V.f Roll}

Roll is the rotation of the structure around the origin O. Roll is caused by the fact that the resultant of the horizontal and vertical wave exciting forces always have a lever arm that will cause a rotational momentum around $\mathrm{O}$. The resultant forces and the lever arms were already shown in figure 6-7.

The roll motion of the floating breakwater in a 2-dimensional plane is described as stated below:

$$
\begin{aligned}
& \left(I+a_{44}\right) \cdot \ddot{\phi}+\left(1 / 2 Y_{w}^{2} \cdot d_{b z}+2 T_{1}^{2} \cdot d_{b y}+b_{44}\right) \cdot \dot{\phi}+\left(1 / 2 Y_{w}^{2} \cdot k_{c z}+2 T_{1}^{2} \cdot k_{c y}+c_{44}+\overline{O G} \cdot m g\right) \cdot \phi=F_{w ; h o r} \cdot z_{b}+F_{w ; v e r t} \cdot y_{b} \\
& \left(I+a_{44}\right) \cdot \ddot{\phi}+\left(1 / 2 Y_{w}^{2} \cdot d_{b z}+2 T_{1}^{2} \cdot d_{b y}+b_{44}\right) \cdot \dot{\phi}+\left(1 / 2 Y_{w}^{2} \cdot k_{c z}+2 T_{1}^{2} \cdot k_{c y}+c_{44}+\overline{O G} \cdot m g\right) \cdot \phi= \\
& -\left\{a_{33} \cdot y_{b} \cdot \ddot{\zeta}_{w 3}\right\}-\left\{b_{33} \cdot y_{b} \cdot \dot{\zeta}_{w 3}\right\}-\left\{c_{33} \cdot C_{3} \cdot y_{b} \cdot \zeta_{w 3}\right\}-\left\{a_{22} \cdot z_{b} \cdot \ddot{\zeta}_{w 2}\right\}-\left\{b_{22} \cdot z_{b} \cdot \dot{\zeta}_{w 2}\right\}
\end{aligned}
$$

\begin{tabular}{|c|c|c|c|c|}
\hline$a_{44}$ & : & Added mass moment of inertia & $\left(\mathrm{Ns}^{2} / \mathrm{rad}\right)$ & \\
\hline$b_{44}$ & : & Hydraulic roll damping & $(\mathrm{Ns} / \mathrm{rad})$ & \\
\hline$c_{44}$ & : & \multicolumn{2}{|l|}{ Water spring stiffness } & $=\rho g Y_{w} \cdot \overline{G M}$ \\
\hline$\overline{G M}$ & : & \multicolumn{2}{|l|}{ Transverse metacentric height } & $=\overline{B M}-\overline{B G}$ \\
\hline & & \multirow{2}{*}{\multicolumn{2}{|c|}{ Distance between centre of buoyancy and metacentre }} & $\frac{1}{12} \cdot Y_{w}^{3}$ \\
\hline$B M$ & $:$ & & & $=\frac{12}{Y_{w} \cdot T_{1}}$ \\
\hline$\overline{B G}$ & : & \multicolumn{3}{|c|}{ Distance between the centres of gravity and buoyancy } \\
\hline$\overline{O G}$ & : & \multicolumn{3}{|c|}{ Distance of centre of gravity below the waterline } \\
\hline$I_{x x}$ & : & \multicolumn{2}{|c|}{ Mass moment of inertia about $\mathrm{G}$ per $\mathrm{m}^{1}$} & $=k_{x x}^{2} \cdot m=\left(0.35 \cdot Y_{w}\right)^{2} \cdot m$ \\
\hline$d_{b y}$ & : & Horizontal mooring damping. & $(\mathrm{Ns} / \mathrm{m}=\mathrm{kg} / \mathrm{s})$ & $=d \cdot \cos \alpha$ \\
\hline$k_{c y}$ & : & Horizontal mooring spring stiffness. & $\left(\mathrm{N} / \mathrm{m}=\mathrm{kg} / \mathrm{s}^{2}\right)$ & $=k \cdot \cos \alpha$ \\
\hline$d_{b z}$ & : & Vertical mooring damping. & $(\mathrm{Ns} / \mathrm{m}=\mathrm{kg} / \mathrm{s})$ & $=d \cdot \sin \alpha$ \\
\hline$k_{c z}$ & : & Vertical mooring spring stiffness. & $\left(\mathrm{N} / \mathrm{m}=\mathrm{kg} / \mathrm{s}^{2}\right)$ & $=k \cdot \sin \alpha$ \\
\hline$\phi$ & : & Rotation about the $\mathrm{x}$-axis & $(\mathrm{rad})$ & $=-\phi_{a} \cos \left(\omega t+\varepsilon_{\phi \zeta}\right)$ \\
\hline$\dot{\phi}$ & : & Velocity of rotation about the $\mathrm{x}$-axis & $(\mathrm{rad} / \mathrm{s})$ & $=\phi_{a} \omega \cdot \sin \left(\omega t+\varepsilon_{\phi \zeta}\right)$ \\
\hline$\ddot{\phi}$ & : & \multicolumn{2}{|c|}{ Acceleration of rotation about the $\mathrm{x}$-axis $\left(\mathrm{rad} / \mathrm{s}^{2}\right)$} & $=\phi_{a} \omega^{2} \cdot \cos \left(\omega t+\varepsilon_{\phi \zeta}\right)$ \\
\hline$\zeta_{w 3}^{*}$ & : & \multicolumn{2}{|c|}{ Depth-related reduced vertical wave elevation. } & $=e^{-k T} \cdot \zeta_{a} \sin (\omega t)$ \\
\hline$\dot{\zeta}_{w 3}^{*}$ & : & \multicolumn{2}{|c|}{ Depth-related vertical velocity of the water particles. } & $=e^{-k T} \cdot \zeta_{a} \cdot \omega \cos (\omega t)$ \\
\hline$\ddot{\zeta}_{w 3}^{*}$ & : & \multicolumn{2}{|c|}{ Depth-related vertical acceleration of the water particles. } & $=-e^{-k T} \cdot \zeta_{a} \cdot \omega^{2} \sin (\omega t)$ \\
\hline$\zeta_{w 2}^{*}$ & : & \multicolumn{2}{|c|}{ Depth-related reduced horizontal wave elevation. } & $=-e^{-k T} \cdot \zeta_{a} \cos (\omega t)$ \\
\hline$\dot{\zeta}_{w 2}^{*}$ & : & \multicolumn{2}{|c|}{ Depth-related horizontal velocity of the water particles. } & $=e^{-k T} \cdot \zeta_{a} \cdot \omega \sin (\omega t)$ \\
\hline$\ddot{\zeta}_{w 2}^{*}$ & : & \multicolumn{2}{|c|}{ Depth-related horizontal acceleration of the water particles. } & $=e^{-k T} \cdot \zeta_{a} \cdot \omega^{2} \cos (\omega t)$ \\
\hline$y_{b}$ & : & \multicolumn{2}{|c|}{ Horizontal lever arm, relative to the origin. } & \\
\hline$z_{b}$ & : & \multicolumn{2}{|l|}{ Vertical lever arm, relative to the origin. } & \\
\hline$\alpha$ & : & Mooring line angle & $\left({ }^{\circ}\right)$ & \\
\hline
\end{tabular}

\section{Calculation Parameters}




\section{Roll motions and forces}

\section{Wave moments about 0}

$$
M_{w}=F_{w ; h o r} \cdot z_{b}+F_{w ; v e r t} \cdot y_{b}
$$

A substitution of B.34 and B.52 in B.75 yields:

$$
\begin{aligned}
M_{w} & =\left[\zeta_{a} \cdot e^{-k T_{2}}\left\{a_{22} \omega^{2}\right\} \cos (\omega t)+\zeta_{a} \cdot e^{-k T_{2}}\left\{b_{22} \omega\right\} \sin (\omega t)\right] \cdot z_{b} \\
& +\left[-\zeta_{a} \cdot e^{-k T_{1}}\left\{c_{33} \cdot C_{3}-a_{33} \omega^{2}\right\} \sin (\omega t)+\zeta_{a} \cdot e^{-k T_{1}}\left\{b_{33} \omega\right\} \cos (\omega t)\right] \cdot y_{b}
\end{aligned}
$$

The wave moment can be separated independently in terms of in-phase and out-of phase terms:

$$
M_{w}=M_{a} \cos \left(\omega t+\varepsilon_{M \zeta}\right)=M_{a} \cos \left(\varepsilon_{M \zeta}\right) \cos (\omega t)+M_{a} \sin \left(\varepsilon_{M \zeta}\right) \sin (\omega t)
$$

Combining the two in-phase and out-of phase terms of equations B.76 and B.77 yields:

$$
\begin{aligned}
& M_{a} \cos \left(\varepsilon_{M \zeta}\right)=\zeta_{a} \cdot\left[e^{-k T_{2}} \cdot z_{b} \cdot\left\{a_{22} \omega^{2}\right\}+e^{-k T_{1}} \cdot y_{b} \cdot\left\{b_{33} \omega\right\}\right] \\
& M_{a} \sin \left(\varepsilon_{M \zeta}\right)=\zeta_{a} \cdot\left[e^{-k T_{2}} \cdot z_{b} \cdot\left\{b_{22} \omega\right\}-e^{-k T_{1}} \cdot y_{b} \cdot\left\{c_{33} \cdot C_{3}-a_{33} \omega^{2}\right\}\right]
\end{aligned}
$$

Adding the squares of these two equations results in the wave moment amplitude at a depth ' $\mathrm{T}$ ' on the construction:

$$
\frac{M_{a}}{\zeta_{a}}=\sqrt{\left[e^{-k T_{2}} \cdot z_{b} \cdot\left\{a_{22} \omega^{2}\right\}+e^{-k T_{1}} \cdot y_{b} \cdot\left\{b_{33} \omega\right\}\right]^{2}+\left[e^{-k T_{2}} \cdot z_{b} \cdot\left\{b_{22} \omega\right\}-e^{-k T_{1}} \cdot y_{b} \cdot\left\{c_{33} \cdot C_{3}-a_{33} \omega^{2}\right\}\right]^{2}}
$$

\section{Roll motion}

A substitution of B.74 and B.76 into the equation of motion of B.70 yields:

$$
\begin{aligned}
& \left\{\left(I+a_{44}\right) \cdot \phi_{a} \omega^{2} \cdot \cos \left(\omega t+\varepsilon_{\phi \zeta}\right)\right\}+\left\{\left(1 / 2 Y_{w}^{2} \cdot d_{b z}+2 T_{1}^{2} \cdot d_{b y}+b_{44}\right) \cdot \phi_{a} \omega \cdot \sin \left(\omega t+\varepsilon_{\phi \zeta}\right)\right\} \\
& -\left\{\left(1 / 2 Y_{w}^{2} \cdot k_{c z}+2 T_{1}^{2} \cdot k_{c y}+c_{44}+\overline{O G} \cdot m g\right) \cdot \phi_{a} \cos \left(\omega t+\varepsilon_{\phi \zeta}\right)\right\}=M_{w}
\end{aligned}
$$

After ordering the factors on sinus and cosinus, this equation becomes:

$$
\begin{aligned}
& \phi_{a}\left\{\left(I+a_{44}\right) \omega^{2}-\left(1 / 2 Y_{w}^{2} \cdot k_{c z}+2 T_{1}^{2} \cdot k_{c y}+c_{44}+\overline{O G} \cdot m g\right)\right\} \cos \left(\omega t+\varepsilon_{\phi \zeta}\right)+\phi_{a}\left\{\left(1 / 2 Y_{w}^{2} \cdot d_{b z}+2 T_{1}^{2} \cdot d_{b y}+b_{44}\right) \omega\right\} \sin \left(\omega t+\varepsilon_{\phi \zeta}\right) \\
& =\zeta_{a} \cdot\left\{\left(e^{-k T_{2}} \cdot z_{b} \cdot a_{22} \omega^{2}\right)+\left(e^{-k T_{1}} \cdot y_{b} \cdot b_{33} \omega\right)\right\} \cos (\omega t)+\zeta_{a} \cdot\left\{\left(e^{-k T_{2}} \cdot z_{b} \cdot b_{22} \omega\right)-\left(y_{b} \cdot e^{-k T_{1}}\left(c_{33} \cdot C_{3}-a_{33} \omega^{2}\right)\right)\right\} \sin (\omega t)
\end{aligned}
$$

Splitting the angle $\left(\omega t+\varepsilon_{\phi \zeta}\right)$ and writing the in-phase and the out-of-phase terms separately:

$\phi_{a}\left\{\left\{\left(I+a_{44}\right) \omega^{2}-\left(1 / 2 Y_{w}^{2} \cdot k_{c z}+2 T_{1}^{2} \cdot k_{c y}+c_{44}+\overline{O G} \cdot m g\right)\right\} \cos \left(\varepsilon_{\phi \zeta}\right)+\left\{\left(1 / 2 Y_{w}^{2} \cdot d_{b z}+2 T_{1}^{2} \cdot d_{b y}+b_{44}\right) \omega\right\} \sin \left(\varepsilon_{\phi \zeta}\right)\right\} \cdot \cos (\omega t)+$

$\phi_{a}\left\{\left\{\left(I+a_{44}\right) \omega^{2}-\left(1 / 2 Y_{w}^{2} \cdot k_{c z}+2 T_{1}^{2} \cdot k_{c y}+c_{44}+\overline{O G} \cdot m g\right)\right\} \sin \left(\varepsilon_{\phi \zeta}\right)+\left\{\left(1 / 2 Y_{w}^{2} \cdot d_{b z}+2 T_{1}^{2} \cdot d_{b y}+b_{44}\right) \omega\right\} \cos \left(\varepsilon_{\phi \zeta}\right)\right\} \cdot \sin (\omega t)=$

$=\zeta_{a} \cdot\left\{\left(e^{-k T_{2}} \cdot z_{b} \cdot a_{22} \omega^{2}\right)+\left(e^{-k T_{1}} \cdot y_{b} \cdot b_{33} \omega\right)\right\} \cos (\omega t)+\zeta_{a} \cdot\left\{\left(e^{-k T_{2}} \cdot z_{b} \cdot b_{22} \omega\right)-\left(y_{b} \cdot e^{-k T_{1}}\left(c_{33} \cdot C_{3}-a_{33} \omega^{2}\right)\right)\right\} \sin (\omega t)$ 
By equating the two out-of-phase terms and the two in-phase terms, one obtains two equations with two unknowns:

$\phi_{a}\left\{\left\{\left(I+a_{44}\right) \omega^{2}-\left(1 / 2 Y_{w}^{2} \cdot k_{c z}+2 T_{1}^{2} \cdot k_{c y}+c_{44}+\overline{O G} \cdot m g\right)\right\} \cos \left(\varepsilon_{\phi \zeta}\right)+\left\{\left(1 / 2 Y_{w}^{2} \cdot d_{b z}+2 T_{1}^{2} \cdot d_{b y}+b_{44}\right) \omega\right\} \sin \left(\varepsilon_{\phi \zeta}\right)\right\}=$

$=\zeta_{a} \cdot\left\{\left(e^{-k T_{2}} \cdot z_{b} \cdot a_{22} \omega^{2}\right)+\left(e^{-k T_{1}} \cdot y_{b} \cdot b_{33} \omega\right)\right\}$

$(B-83)$

$\phi_{a}\left\{\left\{\left(I+a_{44}\right) \omega^{2}-\left(1 / 2 Y_{w}^{2} \cdot k_{c z}+2 T_{1}^{2} \cdot k_{c y}+c_{44}+\overline{O G} \cdot m g\right)\right\} \sin \left(\varepsilon_{\phi \zeta}\right)+\left\{\left(1 / 2 Y_{w}^{2} \cdot d_{b z}+2 T_{1}^{2} \cdot d_{b y}+b_{44}\right) \omega\right\} \cos \left(\varepsilon_{\phi \zeta}\right)\right\}=$

$=\zeta_{a} \cdot\left\{\left(e^{-k T_{2}} \cdot z_{b} \cdot b_{22} \omega\right)-\left(y_{b} \cdot e^{-k T_{1}}\left(c_{33} \cdot C_{3}-a_{33} \omega^{2}\right)\right)\right\}$

Adding the squares of these two equations results in the RAO of the roll amplitude:

$$
\frac{\phi_{a}}{\zeta_{a}}=\sqrt{\frac{\left\{\left(e^{-k T_{2}} \cdot z_{b} \cdot a_{22} \omega^{2}\right)+\left(e^{-k T_{1}} \cdot y_{b} \cdot b_{33} \omega\right)\right\}^{2}+\left\{\left(e^{-k T_{2}} \cdot z_{b} \cdot b_{22} \omega\right)-\left(y_{b} \cdot e^{-k T_{1}}\left(c_{33} \cdot C_{3}-a_{33} \omega^{2}\right)\right)\right\}^{2}}{\left\{\left(I+a_{44}\right) \omega^{2}-\left(1 / 2 Y_{w}^{2} \cdot k_{c z}+2 T_{1}^{2} \cdot k_{c y}+c_{44}+\overline{O G} \cdot m g\right)\right\}^{2}+\left\{\left(1 / 2 Y_{w}^{2} \cdot d_{b z}+2 T_{1}^{2} \cdot d_{b y}+b_{44}\right) \omega\right\}^{2}}}
$$

The roll motion amplitude $(\mathrm{deg} / \mathrm{m})$ for several wave frequencies is shown in figure B-29. When the roll amplitude is compared with the DELFRAC calculation, some difference is visible.

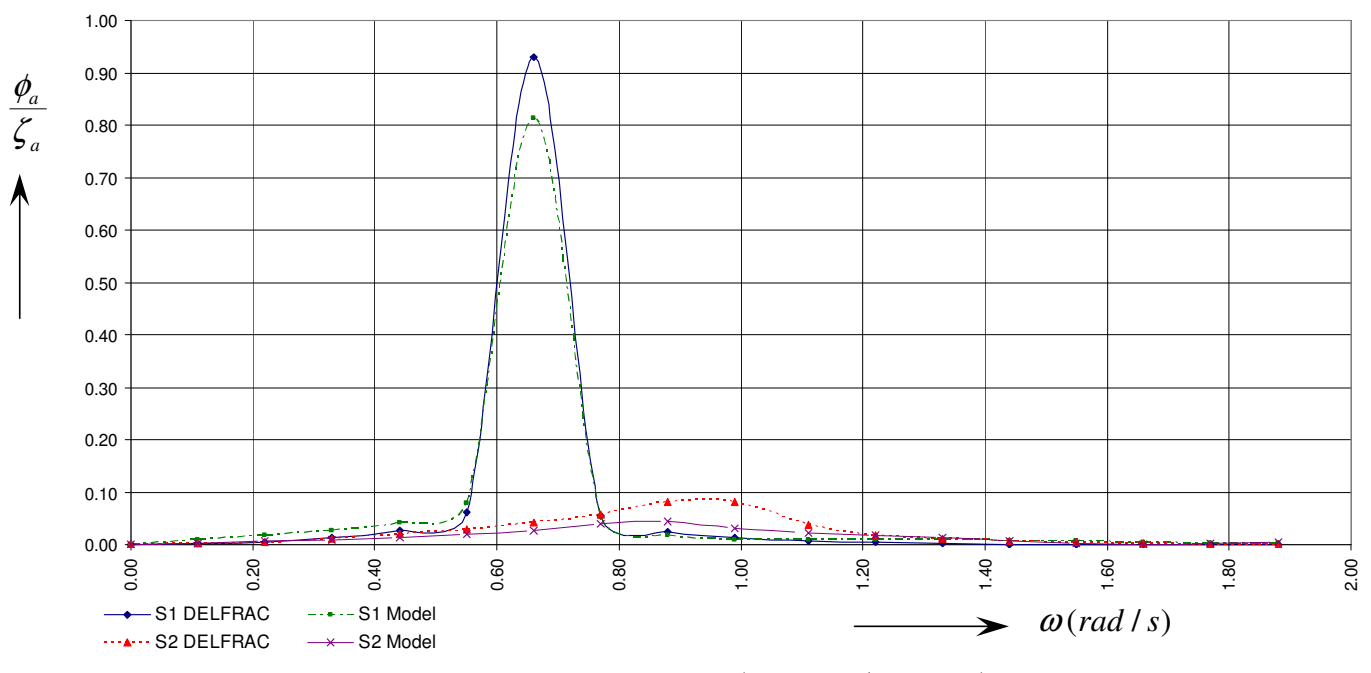

Figure B-29: Roll reaction at several wave frequencies $\left(T_{1}=7.8 \mathrm{~m} / Y_{w}=25 \mathrm{~m}\right)$.

Elimination of $\phi_{a} / \zeta_{a}$ in the equations of B.83 leads to the phase shift:

$\varepsilon_{\phi \zeta}=\arctan \left\{\frac{A \cdot D-C \cdot B}{C \cdot A-B \cdot D}\right\}$

Where:

$$
\begin{array}{ll}
A: \quad & \left\{\left(I+a_{44}\right) \omega^{2}-\left(1 / 2 Y_{w}^{2} \cdot k_{c z}+2 T_{1}^{2} \cdot k_{c y}+c_{44}+\overline{O G} \cdot m g\right)\right\} \\
B: \quad & \left\{\left(1 / 2 Y_{w}^{2} \cdot d_{b z}+2 T_{1}^{2} \cdot d_{b y}+b_{44}\right) \omega\right\} \\
C: \quad & \left\{\left(e^{-k T_{2}} \cdot z_{b} \cdot a_{22} \omega^{2}\right)+\left(e^{-k T_{1}} \cdot y_{b} \cdot b_{33} \omega\right)\right\} \\
D: & \left\{\left(e^{-k T_{2}} \cdot z_{b} \cdot b_{22} \omega\right)-\left(y_{b} \cdot e^{-k T_{1}}\left(c_{33} \cdot C_{3}-a_{33} \omega^{2}\right)\right)\right\}
\end{array}
$$

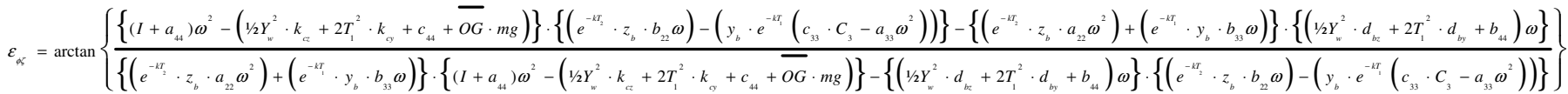


With: $0 \leq \varepsilon_{\phi \zeta} \leq 2 \pi$

The lever arm, relative to $\mathrm{G}$, of the vertical wave force is:

$A_{1}=A_{2} \rightarrow \int_{0}^{1 / 2 y_{b}} \sin (\omega t) d y=\int_{1 / 2 y_{b}}^{1 / 2 Y_{w}} \sin (\omega t) d y$

$-\cos \left(1 / 2 y_{b}\right)+1=-\cos \left(1 / 2 Y_{w}\right)+\cos \left(1 / 2 y_{b}\right)$

$y_{b}=\frac{\cos ^{-1}\left(\frac{1+\cos \left(\frac{Y_{w}}{L} \pi\right)}{2}\right)}{\pi} \cdot L$

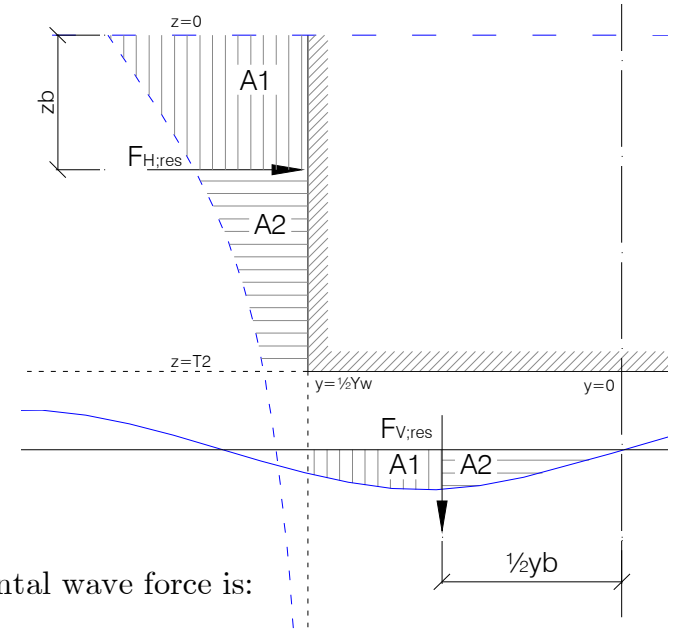

The lever arm, relative to the mean water line, of the horizontal wave force is:

$$
\begin{aligned}
& A_{1}=A_{2} \rightarrow \int_{0}^{z_{b}} e^{-k z} d z=\int_{z_{b}}^{T_{2}} e^{-k z} d z \\
& -\frac{1}{k} e^{-k z_{b}}+\frac{1}{k}=-\frac{1}{k} e^{-k T_{2}}+\frac{1}{k} e^{-k z_{b}} \\
& z_{b}=\frac{\ln \left(\frac{1+e^{-k \cdot T_{2}}}{2}\right)}{-k}
\end{aligned}
$$

(B-87) 


\section{Energy Consideration}

Suppose the breakwater is carrying out a harmonic oscillation:

$\phi=\phi_{a} \sin \omega t$

To understand the amount of energy the roll motion of the floating breakwater passes on to the still water level at the harbour-side, one has to determine the amount of work done by the mass, damping and spring force components per unit of time during one period of oscillation $\mathrm{T}$.

Mass:

$$
E_{m}=\frac{1}{T} \int_{0}^{T}\left\{\left(I+a_{44}\right) \cdot \ddot{\phi}\right\} \cdot\{\dot{\phi} \cdot d t\}=\frac{-\phi_{a}^{2}\left(I+a_{44}\right) \omega^{3}}{T} \int_{0}^{T} \cos (\omega t) \cdot \sin (\omega t) \cdot d t=0
$$

Damping:

$$
E_{d}=\frac{1}{T} \int_{0}^{T}\left\{b_{44} \cdot \dot{\phi}\right\} \cdot\{\dot{\phi} \cdot d t\}=\frac{\phi_{a}^{2} b_{44} \omega^{2}}{T} \int_{0}^{T} \cos ^{2}(\omega t) \cdot d t=\frac{1}{2} b_{44} \omega^{2} \phi_{a}^{2}
$$

Spring

$$
E_{s}=\frac{1}{T} \int_{0}^{T}\left\{c_{44} \cdot \phi\right\} \cdot\{\dot{\phi} \cdot d t\}=\frac{\phi_{a} c_{44} \omega}{T} \int_{0}^{T} \sin (\omega t) \cdot \cos (\omega t) \cdot d t=0 .
$$

Only the damping force $(b \cdot \dot{\phi})$ dissipates energy, in other words: damping is the reason why the motion of oscillation dies out. This damping energy is exactly the amount of energy which is dissipated by the waves on both sides of the structure. The wave energy per unit area times the covered distance by the radiated wave energy in one period divided by the time gives:

$$
E=\frac{1}{T} \cdot 2 \cdot L_{w} \cdot\left\{\frac{1}{2} \rho g \zeta_{c}^{2}\right\} \cdot\left\{c_{g} \cdot T\right\}=\frac{L_{w} \cdot \rho g^{2} \zeta_{c}^{2}}{2 \omega}
$$

Where:

$$
\begin{array}{lll}
\frac{1}{2} \rho g \zeta_{c}^{2} & : & \text { Wave energy per unit area } \\
c_{g}=\frac{g}{2 \omega} & : & \text { Group velocity (deep water) }
\end{array}
$$

Equalising equations B.88 and B.89 gives the (vertical) transmitted wave due to the roll motion.

$$
\zeta_{T ; \text { roll }}(t)=-\sqrt{\frac{b_{44} \cdot \omega^{3} \cdot \phi_{a}^{2}}{L_{w} \cdot \rho \cdot g^{2}}} \cdot \sin \left(\omega t+\varepsilon_{\phi \zeta}\right)
$$

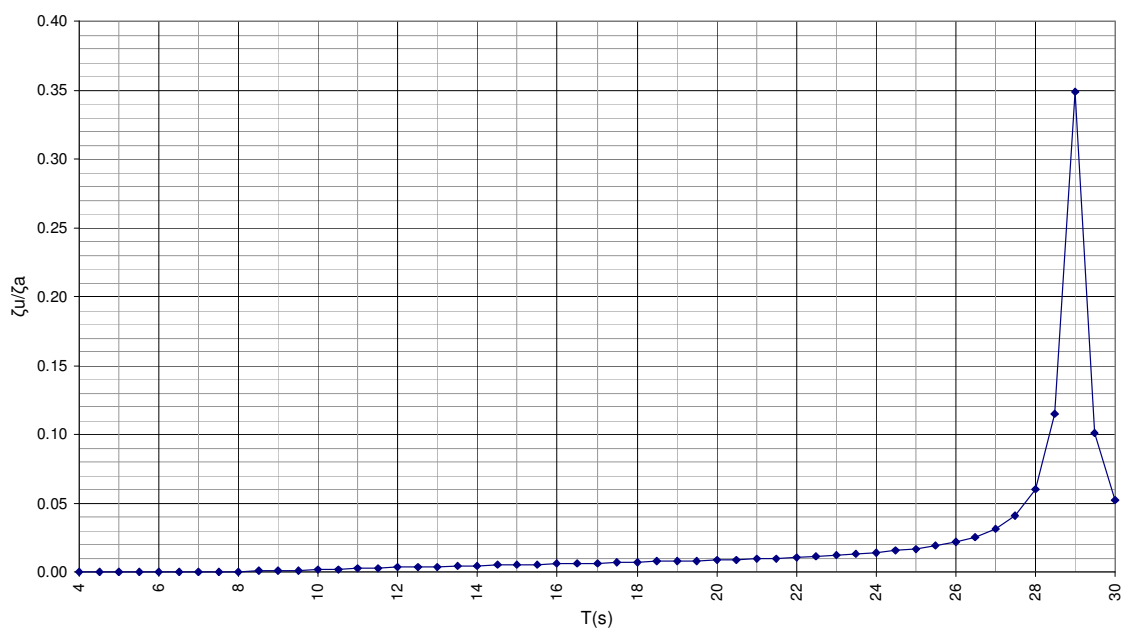

Figure B-30: Wave transmission due to the roll motion $\left(T_{1}=15 \mathrm{~m} / Y_{w}=65 \mathrm{~m}\right)$.

The sway factors in table B-7 become:

$$
\vartheta_{\phi_{a}} \quad: \quad \text { Roll motion amplitude factor } \quad=\sqrt{\frac{b_{44} \cdot \omega^{3} \cdot \phi_{a}^{2}}{L_{w} \cdot \rho \cdot g^{2}}}
$$




\section{B.VI Total transmitted wave amplitude}

The wave amplitude at the harbour side of the floating breakwater is a summation of the waves, produced by the mechanism as they were described in the previous sections.

$$
\zeta_{T}=\left|\zeta_{T ; \text { heave }}+\zeta_{T ; U}+\zeta_{T ; \text { sway }}+\zeta_{T ; \text { roll }}\right| \leq \zeta_{T ; s}
$$

In which $\zeta_{T: s}$ represents the maximum allowed harbour wave amplitude. This wave amplitude depends on the type of ship and the maximum allowed vertical motion $\left(z_{s}\right)$.

$$
\zeta_{T ; s}=\frac{z_{s}}{e^{-k T_{s}} \cdot \sqrt{\frac{\left\{c_{s}-a_{s} \omega^{2}\right\}^{2}+\left\{b_{s} \omega\right\}^{2}}{\left\{c_{s}-\left(m_{s}+a_{s}\right) \omega^{2}\right\}^{2}+\left\{b_{s} \omega\right\}^{2}}}}
$$

When equations B.46, B.64, B.69, B.90 and B.93 are put into equation B.92, the equation for the total transmitted wave amplitude is obtained.

$$
\begin{aligned}
& \left.\zeta_{T}=\mid \sqrt{\frac{b_{33} \cdot \omega^{3} \cdot z_{a}^{2}}{L_{w} \cdot \rho \cdot g^{2}}} \cos \left(\varepsilon_{z \zeta}+\varepsilon_{t o t ; \text { max }}\right)+\sqrt{e^{-k\left(T_{2}-z_{a}\right)} \cdot \zeta_{a}^{2}}+\sqrt{\frac{b_{22} \cdot \omega^{3} \cdot y_{a}^{2}}{L_{w} \cdot \rho \cdot g^{2}}} \cdot \sin \left(\varepsilon_{y \zeta}+\varepsilon_{t o t ; \max }\right)+\sqrt{\frac{b_{44} \cdot \omega^{3} \cdot \phi_{a}^{2}}{L_{w} \cdot \rho \cdot g^{2}} \cdot \sin \left(\varepsilon_{\phi \zeta}\right.}+\varepsilon_{t o t ; \max }\right) \mid \leq \\
& \leq \frac{z_{s}}{e^{-k T_{s}} \cdot \sqrt{\frac{\left\{c_{s}-a_{s} \omega^{2}\right\}^{2}+\left\{b_{s} \omega\right\}^{2}}{\left\{c_{s}-\left(m_{s}+a_{s}\right) \omega^{2}\right\}^{2}+\left\{b_{s} \omega\right\}^{2}}}}
\end{aligned}
$$

Where:

$$
\begin{aligned}
& T_{1}=\frac{m}{\rho \cdot Y_{w}} \\
& T_{2}=T_{\text {screen }}+T_{1} \quad: \text { The point where the maximum total transmitted wave amplitude occurs (paragraph B.V.a). } \\
& \mathcal{E}_{\text {tot } \text {; } \max } \quad \text {. }
\end{aligned}
$$

The total RAO at the harbour side of the floating breakwater becomes:

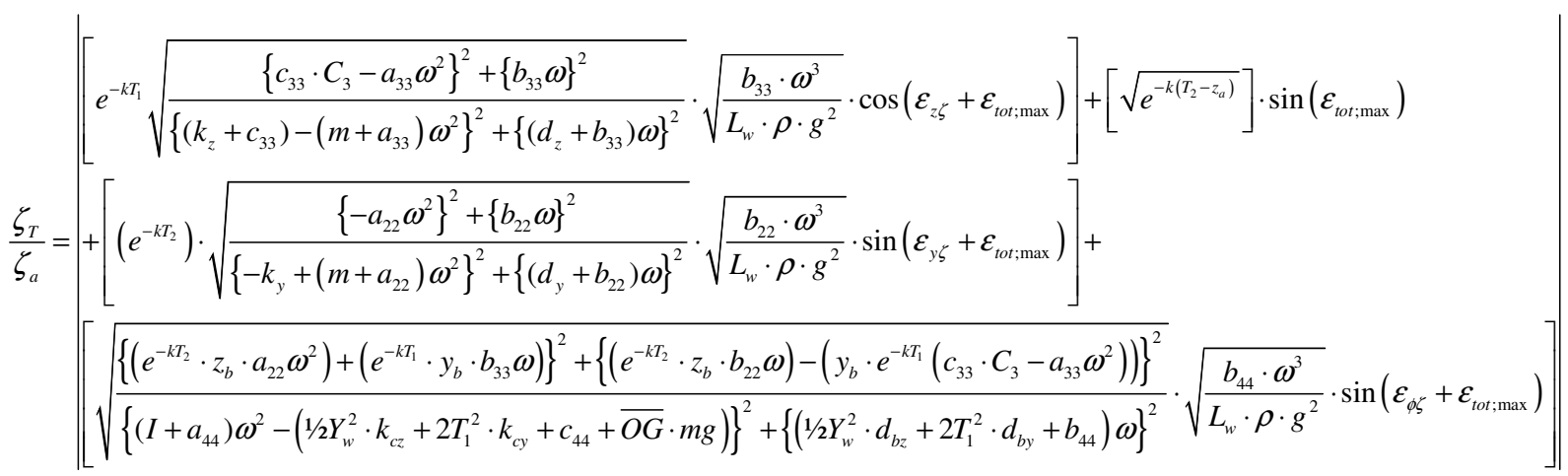




\section{Appendix $C$}

\section{Hydrodynamic evaluation}

\section{C.I Structural variables}

The structures as they were calculated are listed in the table below

\begin{tabular}{|c|c|c|c|c|c|c|c|c|c|c|c|}
\hline \multirow{3}{*}{$\begin{array}{l}\text { Structure } \\
\text { number. }\end{array}$} & Length & Width & $\begin{array}{c}\text { Floater } \\
\text { draft }\end{array}$ & $\begin{array}{c}\text { Width/ } \\
\text { draft }\end{array}$ & $\begin{array}{c}\text { Screen } \\
\text { draft }\end{array}$ & Mass & $\begin{array}{c}\text { Mass moment } \\
\text { of inertia }\end{array}$ & $\begin{array}{l}\text { Centre of gravity } \\
\text { below waterline }\end{array}$ & $\begin{array}{l}\text { Mooring } \\
\text { stiffness }\end{array}$ & $\begin{array}{l}\text { Mooring } \\
\text { damping }\end{array}$ & $\begin{array}{c}\text { Mooring } \\
\text { angle }\end{array}$ \\
\hline & $L_{w}$ & $Y_{w}$ & $T_{1}$ & $Y_{w} / T_{1}$ & $T_{2}$ & $\mathrm{~m}$ & $\mathrm{I}_{\mathrm{xx}}$ & $\overline{O G}$ & $k$ & $d$ & $\alpha$ \\
\hline & $m$ & $m$ & $m$ & - & $m$ & $t$ & t. $m^{2}$ & $m$ & $\mathrm{kN} / \mathrm{m}$ & $k N s / m$ & deg. \\
\hline S1 & 500 & 10 & 10.0 & 1.00 & 10 & 51,250 & $0.63 \cdot 10^{6}$ & 3.05 & - & - & - \\
\hline S2 & 500 & 20 & 10.0 & 2.00 & 10 & 102,500 & $5.02 \cdot 10^{6}$ & 3.05 & - & - & - \\
\hline S3 & 500 & 40 & 10.0 & 4.00 & 10 & 205,000 & $40.18 \cdot 10^{6}$ & 3.05 & - & - & - \\
\hline $\mathrm{S} 4$ & 500 & 60 & 10.0 & 6.00 & 10 & 307,500 & $135.61 \cdot 10^{6}$ & 3.05 & - & - & - \\
\hline S5 & 500 & 80 & 10.0 & 8.00 & 10 & 410,000 & $346.16 \cdot 10^{6}$ & 3.05 & - & - & - \\
\hline S6 & 500 & 10 & 24.0 & 0.42 & 24.0 & 123,000 & $1.51 \cdot 10^{6}$ & 10.05 & - & - & - \\
\hline S7 & 500 & 20 & 12.0 & 1.67 & 12.0 & 123,000 & $6.03 \cdot 10^{6}$ & 4.05 & - & - & - \\
\hline S8 & 500 & 40 & 6.0 & 6.67 & 6.0 & 123,000 & $24.11 \cdot 10^{6}$ & 1.05 & - & - & - \\
\hline S9 & 500 & 60 & 4.0 & 15.00 & 4.0 & 123,000 & $54.24 \cdot 10^{6}$ & 0.05 & - & - & - \\
\hline S10 & 500 & 80 & 3.0 & 26.67 & 3.0 & 123,000 & $96.43 \cdot 10^{6}$ & -0.45 & - & - & - \\
\hline S11 & 500 & 40 & 5 & 8.00 & 5 & 102,500 & $20.09 \cdot 10^{6}$ & 0.55 & - & - & - \\
\hline S12 & 500 & 40 & 20 & 2.00 & 20 & 410,000 & $80.36 \cdot 10^{6}$ & 8.05 & - & - & - \\
\hline S13 & 500 & 40 & 30 & 1.33 & 30 & 615,000 & $120.54 \cdot 10^{6}$ & 13.05 & - & - & - \\
\hline S14 & 500 & 40 & 40 & 1.00 & 40 & 820,000 & $160.72 \cdot 10^{6}$ & 18.05 & - & - & - \\
\hline S15 & 500 & 40 & 10.0 & 4.00 & 15 & 205.000 & $40.18 \cdot 10^{6}$ & 3.05 & - & - & - \\
\hline S16 & 500 & 40 & 10.0 & 4.00 & 20 & 205.000 & $40.18 \cdot 10^{6}$ & 3.05 & - & - & - \\
\hline S17 & 500 & 40 & 10.0 & 4.00 & 25 & 205.000 & $40.18 \cdot 10^{6}$ & 3.05 & - & - & - \\
\hline S18 & 500 & 40 & 10.0 & 4.00 & 35 & 205.000 & $40.18 \cdot 10^{6}$ & 3.05 & - & - & - \\
\hline S19 & 500 & 40 & 10.0 & 4.00 & 10.0 & 205.000 & $40.18 \cdot 10^{6}$ & 3.05 & 50,000 & - & - \\
\hline $\mathrm{S} 20$ & 500 & 40 & 10.0 & 4.00 & 10.0 & 205.000 & $40.18 \cdot 10^{6}$ & 3.05 & 100,000 & - & - \\
\hline $\mathrm{S} 21$ & 500 & 40 & 10.0 & 4.00 & 10.0 & 205.000 & $40.18 \cdot 10^{6}$ & 3.05 & 200,000 & - & - \\
\hline S22 & 500 & 40 & 10.0 & 4.00 & 10.0 & 205.000 & $40.18 \cdot 10^{6}$ & 3.05 & 300,000 & - & - \\
\hline S23 & 50 & 40 & 10.0 & 4.00 & 10.0 & 20,500 & $4.02 \cdot 10^{6}$ & 3.05 & - & - & - \\
\hline S24 & 100 & 40 & 10.0 & 4.00 & 10.0 & 41,000 & $8.04 \cdot 10^{6}$ & 3.05 & - & - & - \\
\hline $\mathrm{S} 25$ & 200 & 40 & 10.0 & 4.00 & 10.0 & 82,000 & $16.07 \cdot 10^{6}$ & 3.05 & - & - & - \\
\hline S26 & 300 & 40 & 10.0 & 4.00 & 10.0 & 123,000 & $24.11 \cdot 10^{6}$ & 3.05 & - & - & - \\
\hline
\end{tabular}




\section{C.II Calculation evaluation}

\section{C.II.a Structural width}

Sway

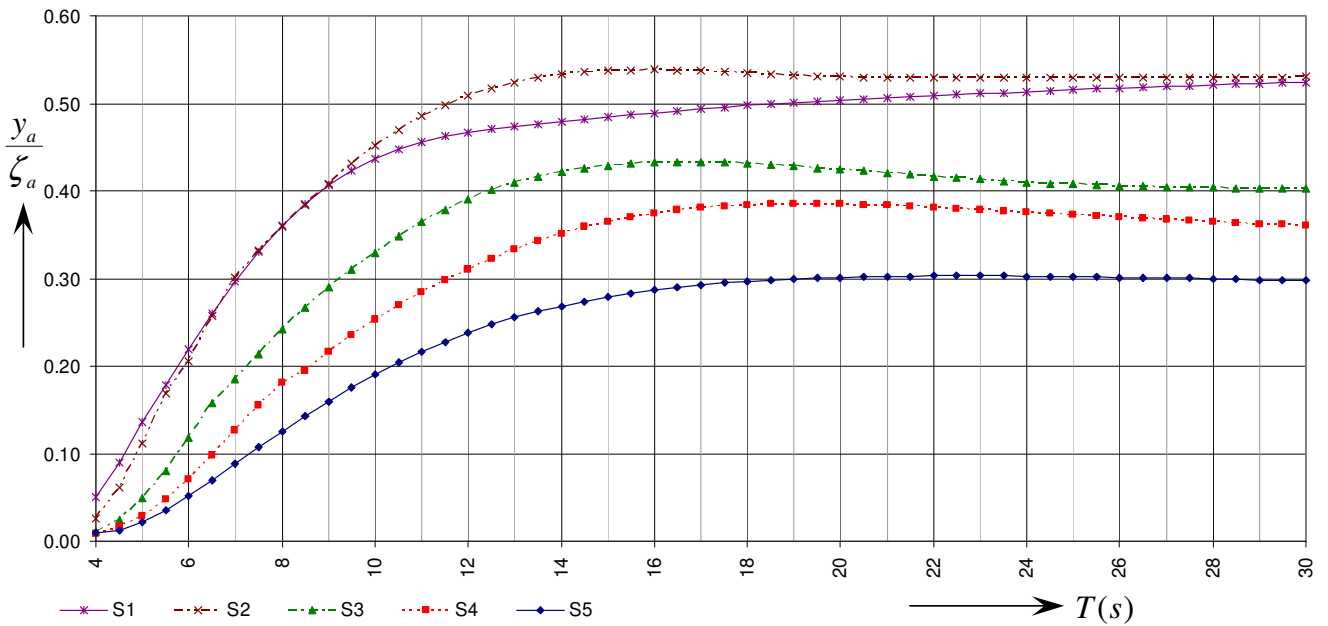

Figure C-1: Sway reaction for structures S1-S5.

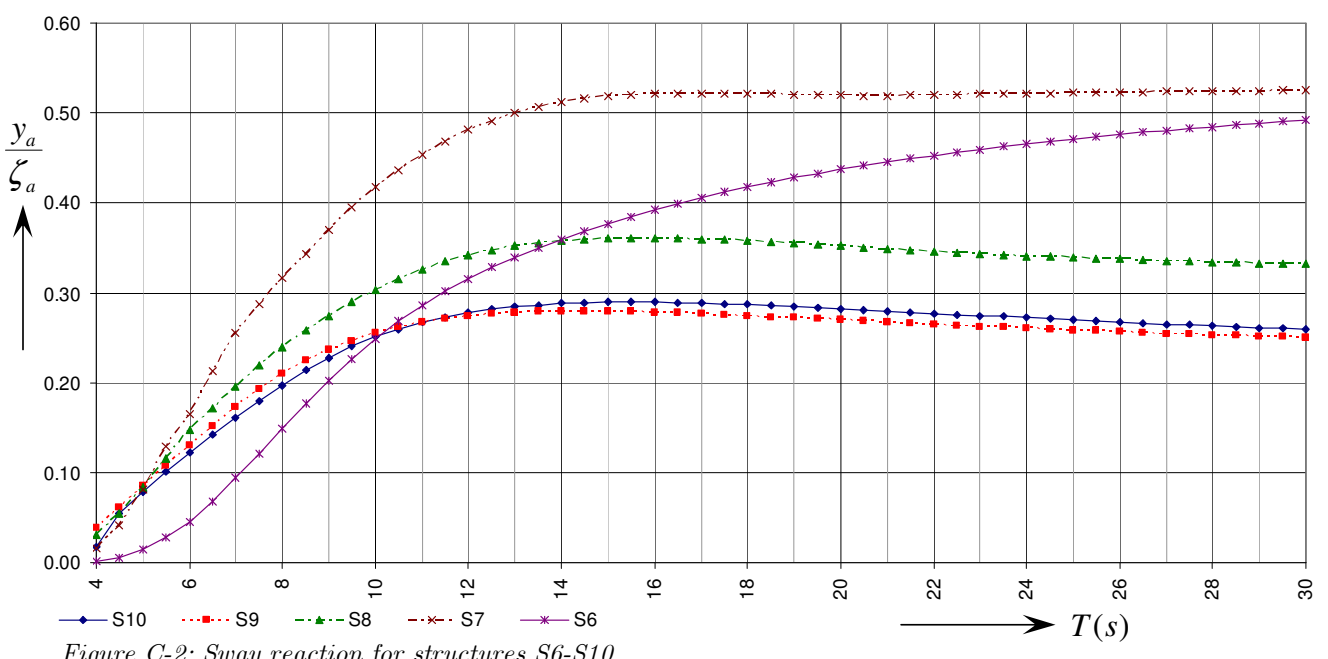

Figure C-2: Sway reaction for structures S6-S10. 


\section{Heave}

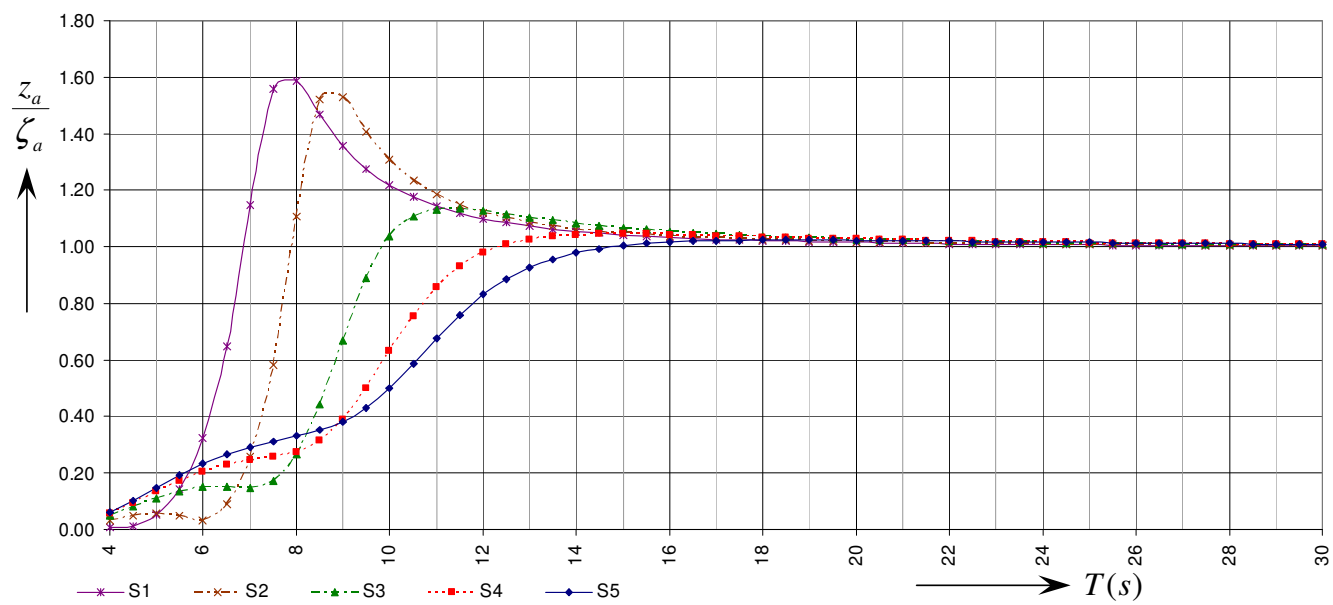

Figure C-3: Heave reaction for structures S1-S5.

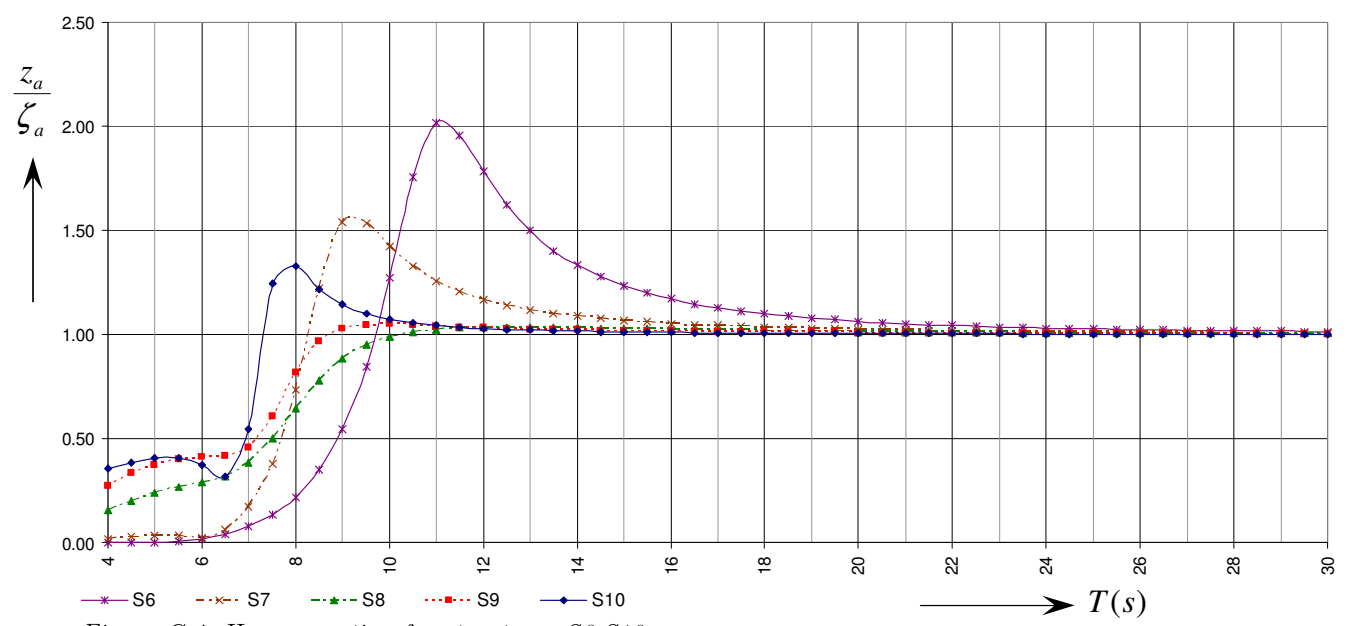

Figure C-4: Heave reaction for structures S6-S10. 
Roll

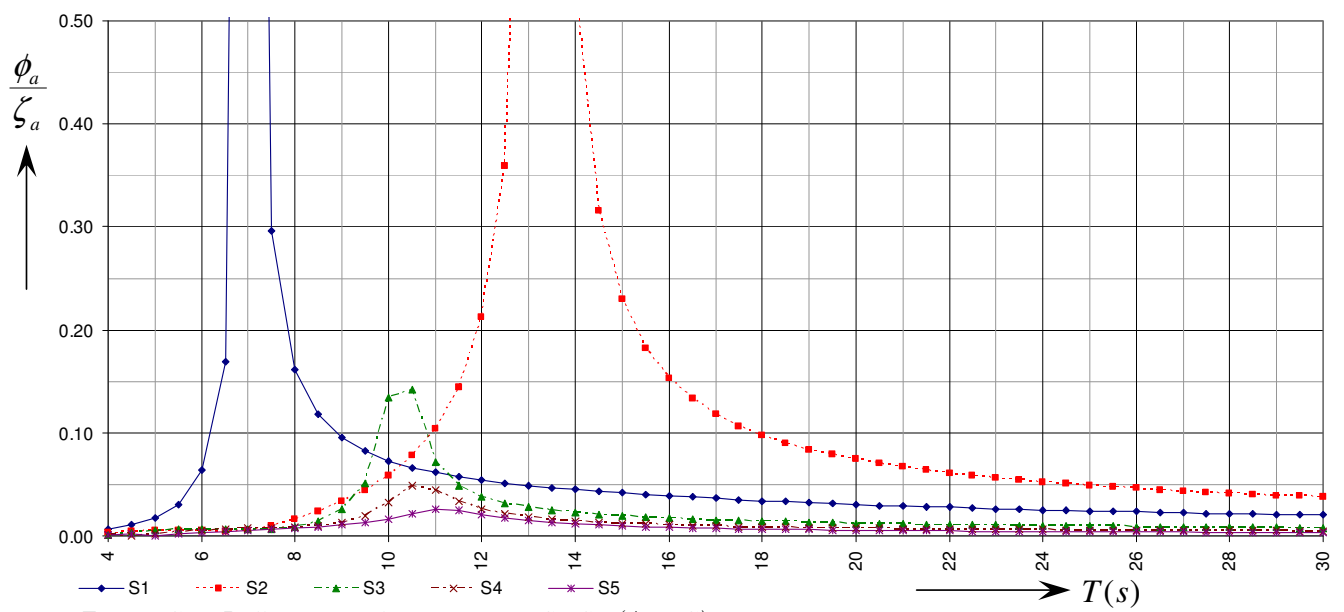

Figure C-5: Roll reaction for structures S1-S5 (App.C)

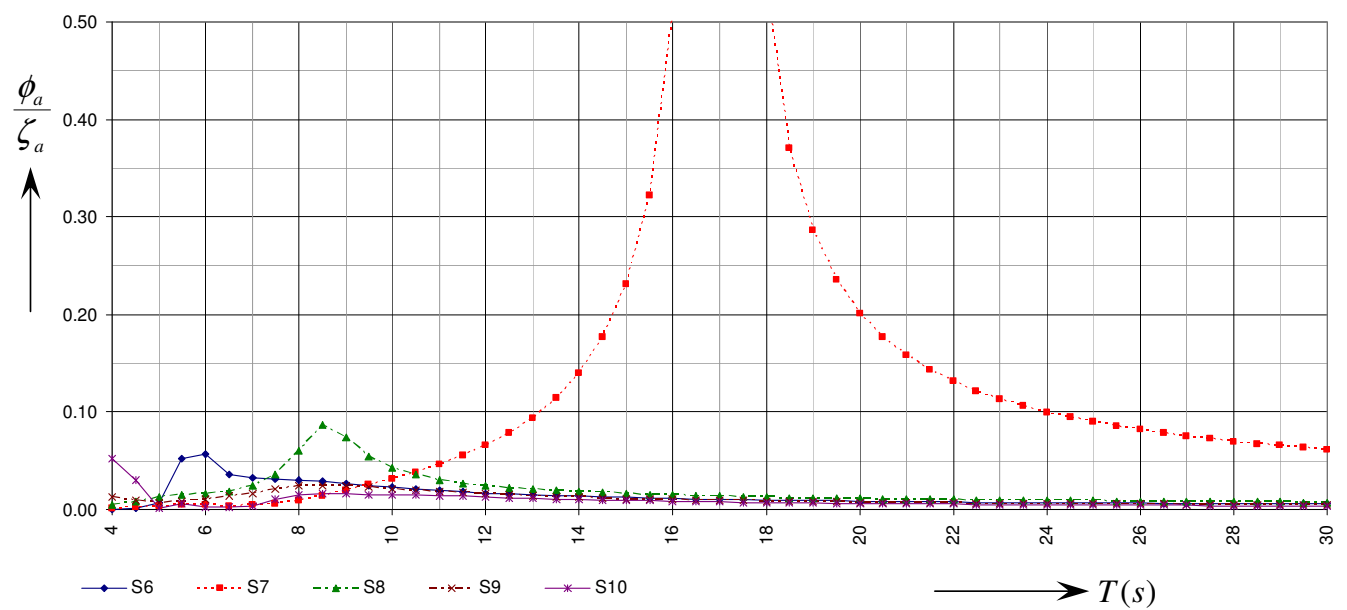

Figure C-6: Roll reaction for structures S6-S10 (App.C) 


\section{C.II.b Draft / Mass}

\section{Sway}

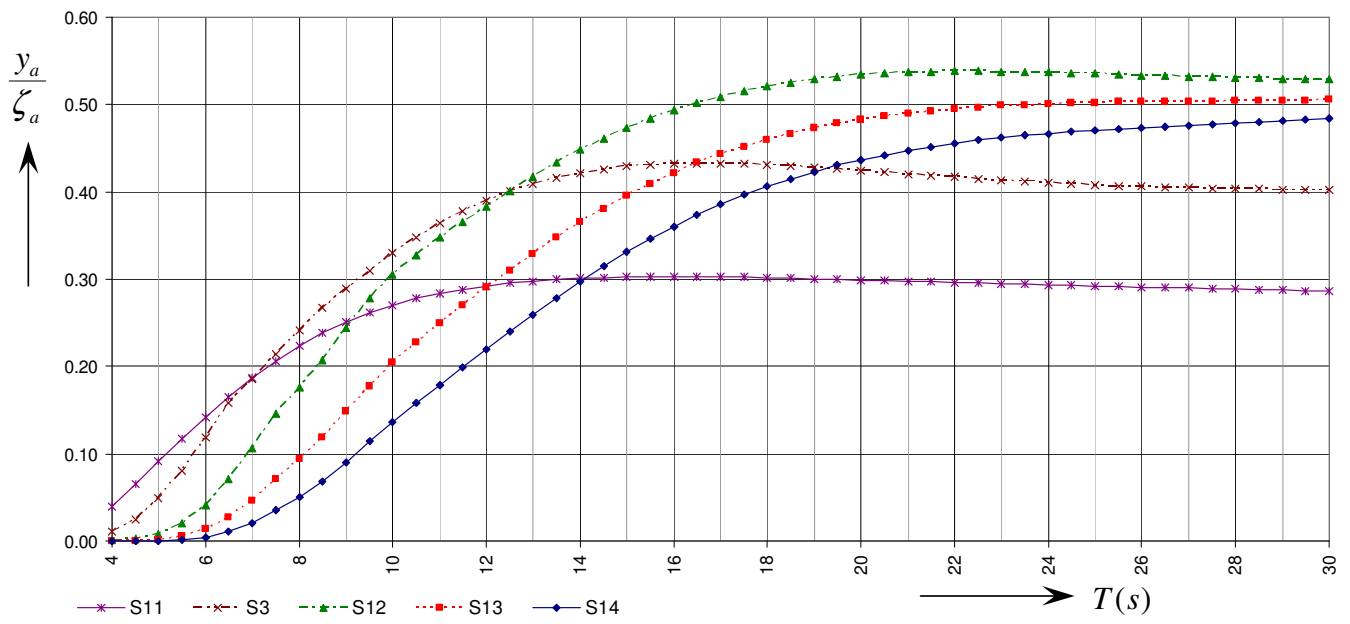

Figure C-7: Sway reaction for structures S3 and S11-S14.

\section{Heave}

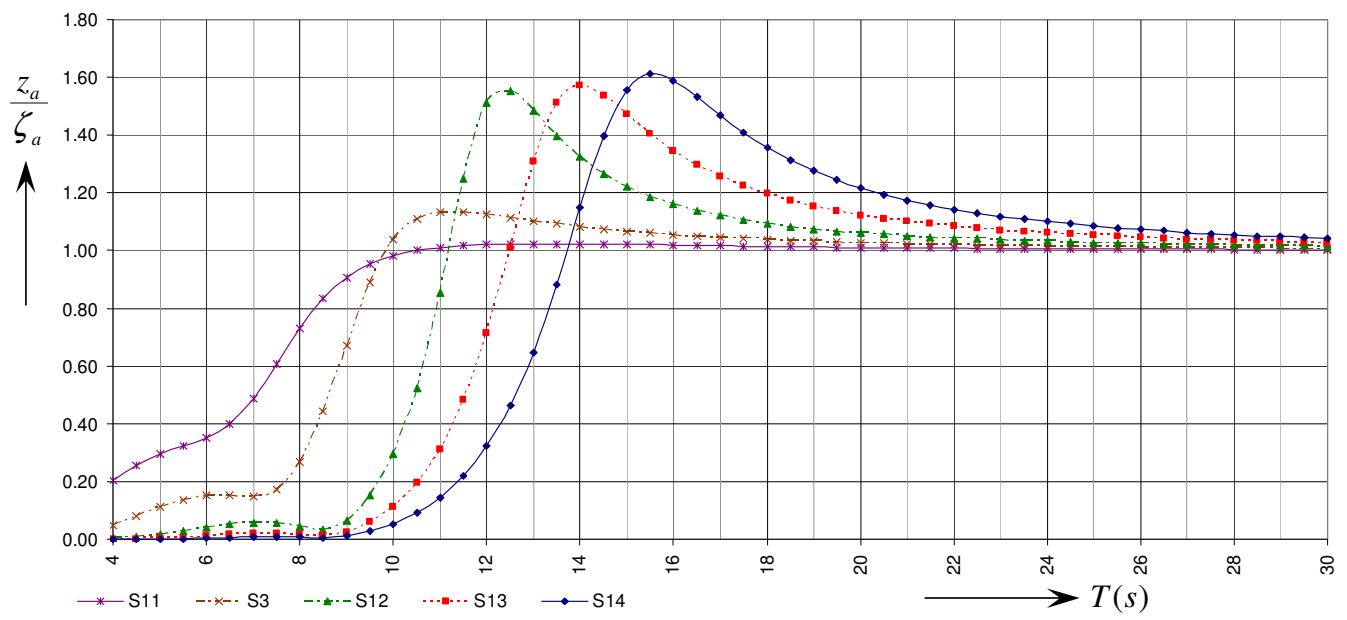

Figure C-8: Heave reaction for structures S3 and S11-S14.

Roll

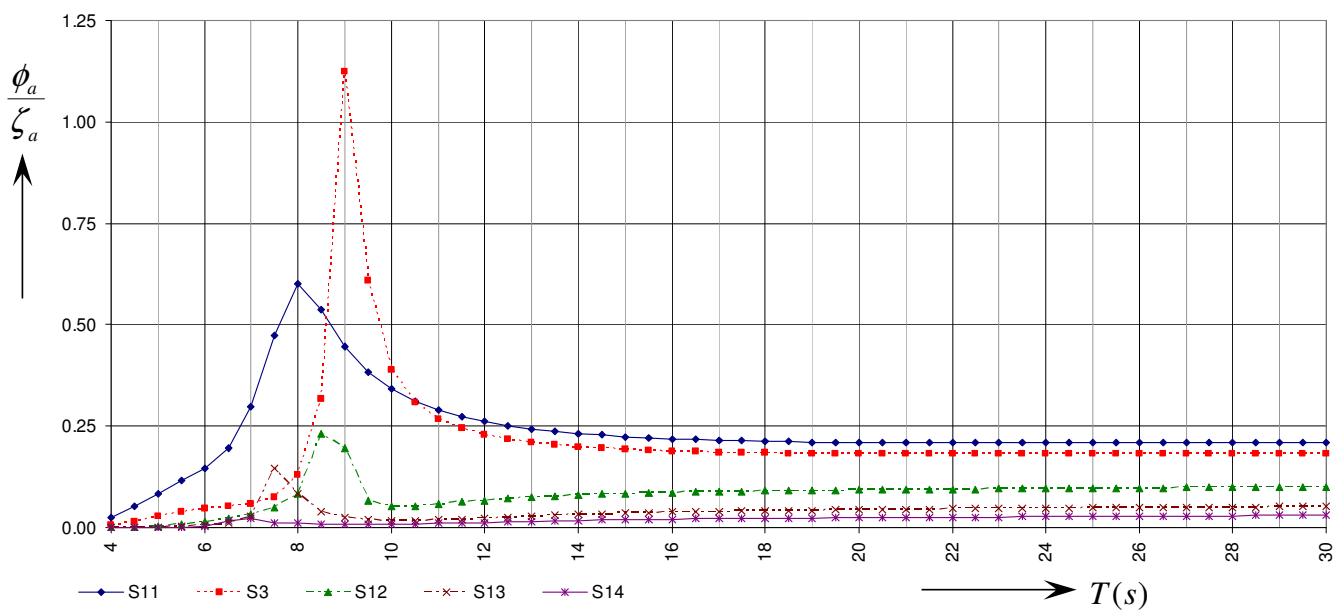

Figure C-9: Roll reaction for structures S3 and S11-S14. 


\section{Underflow}

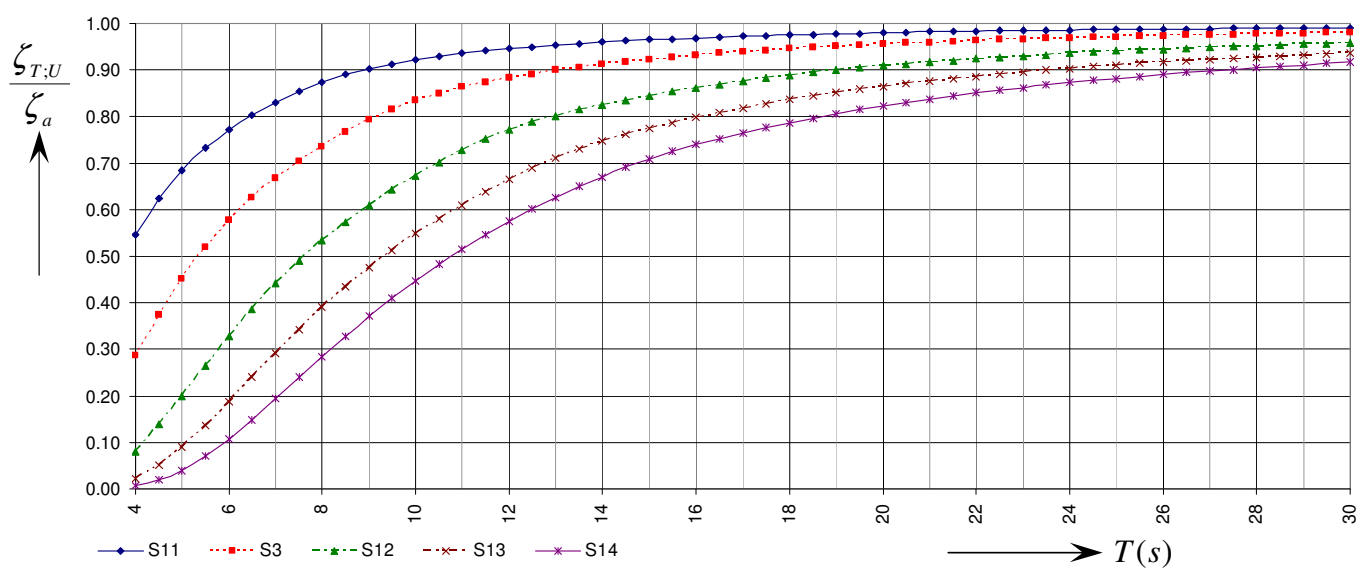

Figure C-10: RAO of underflow for structures S3 and S11-S14. 


\section{C.II.c Screen underneath the floating element}

Sway

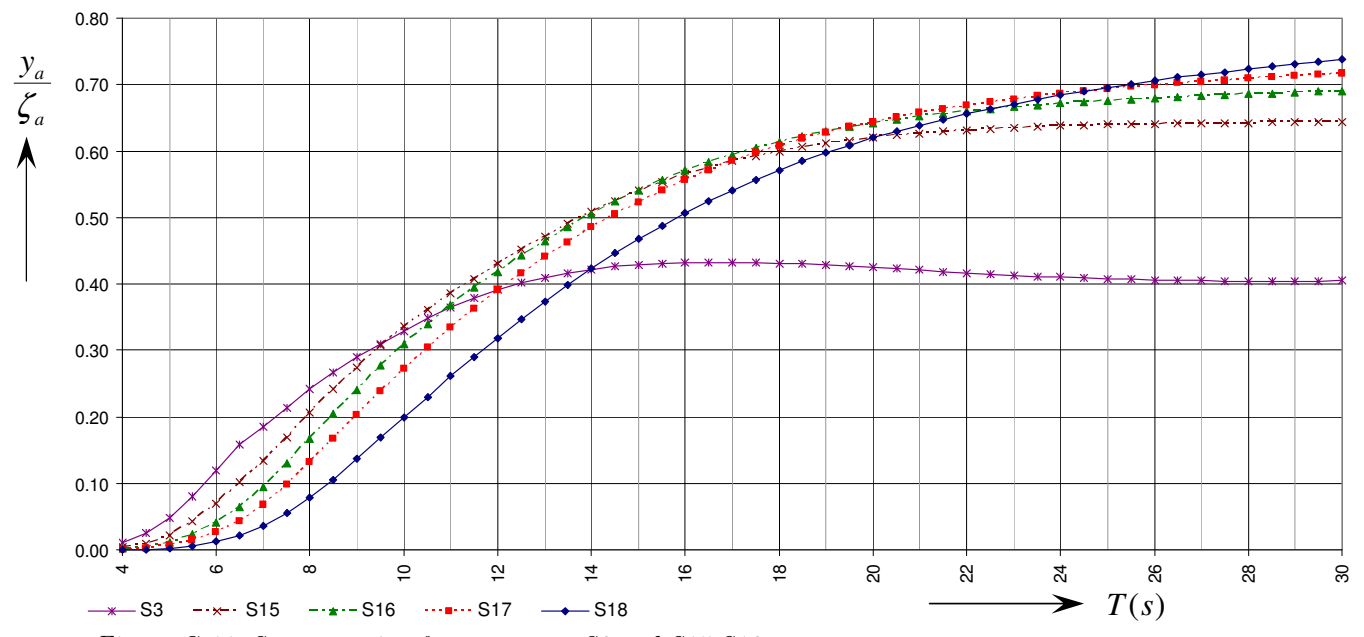

Figure C-11: Sway reaction for structures S3 and S15-S18.

Roll

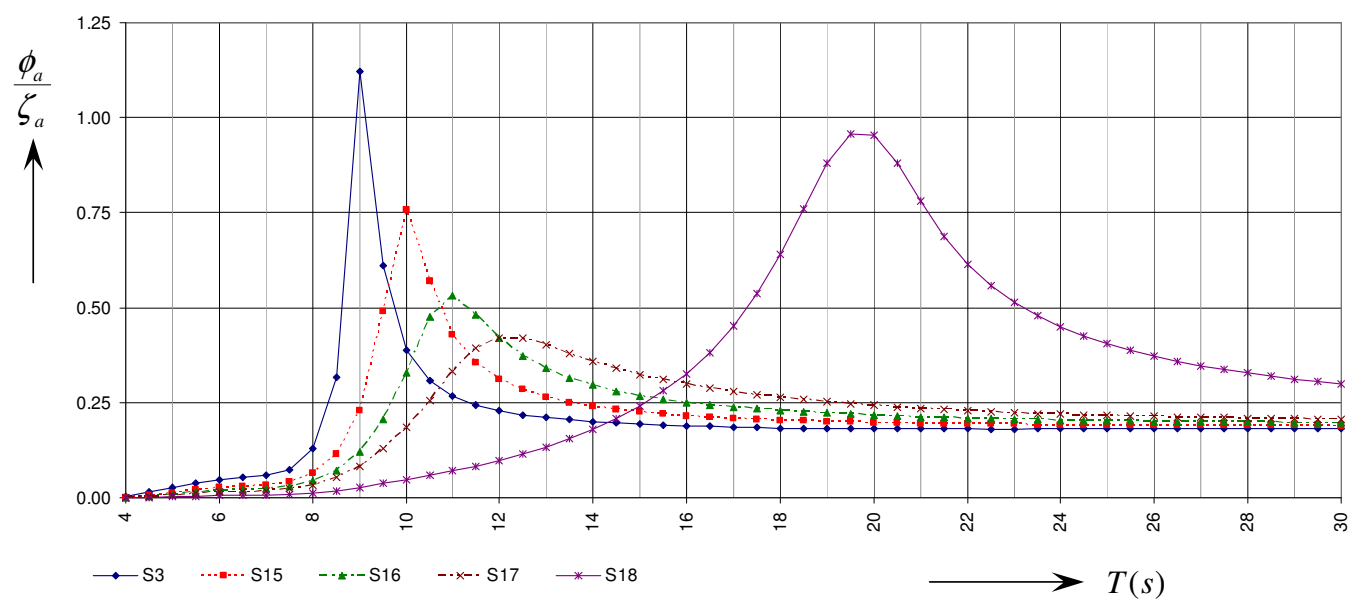

Figure C-12: Roll reaction for structures S3 and S15-S18.

\section{Underflow}

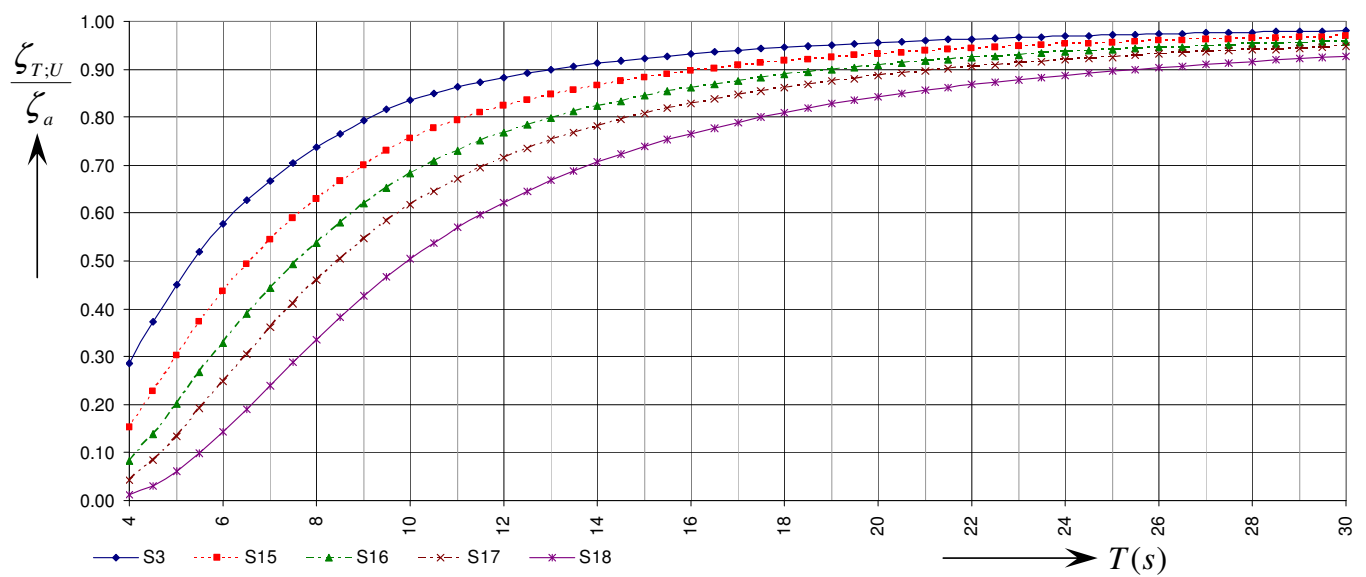

Figure C-13: RAO of underflow for structures S3 and S15-S18. 


\section{C.II.d Mooring line stiffness}

Sway

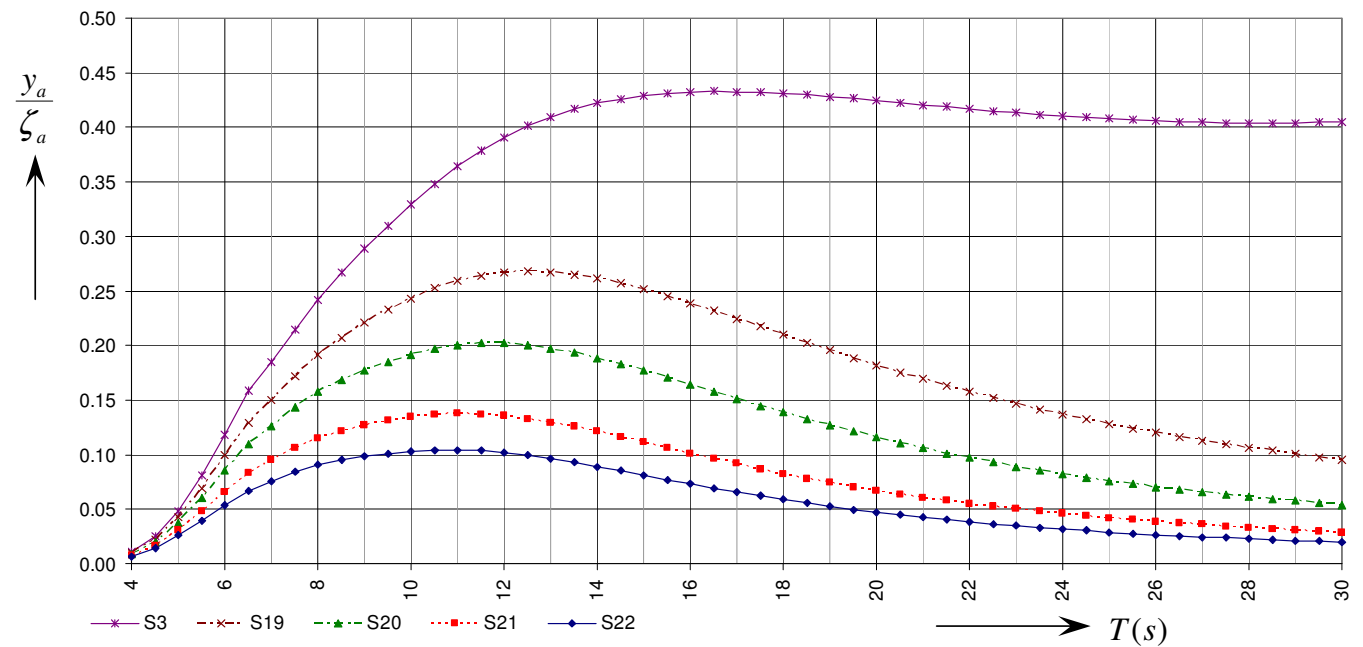

Figure C-14: Sway reaction for structures S3 and S19-S22.

\section{Heave}

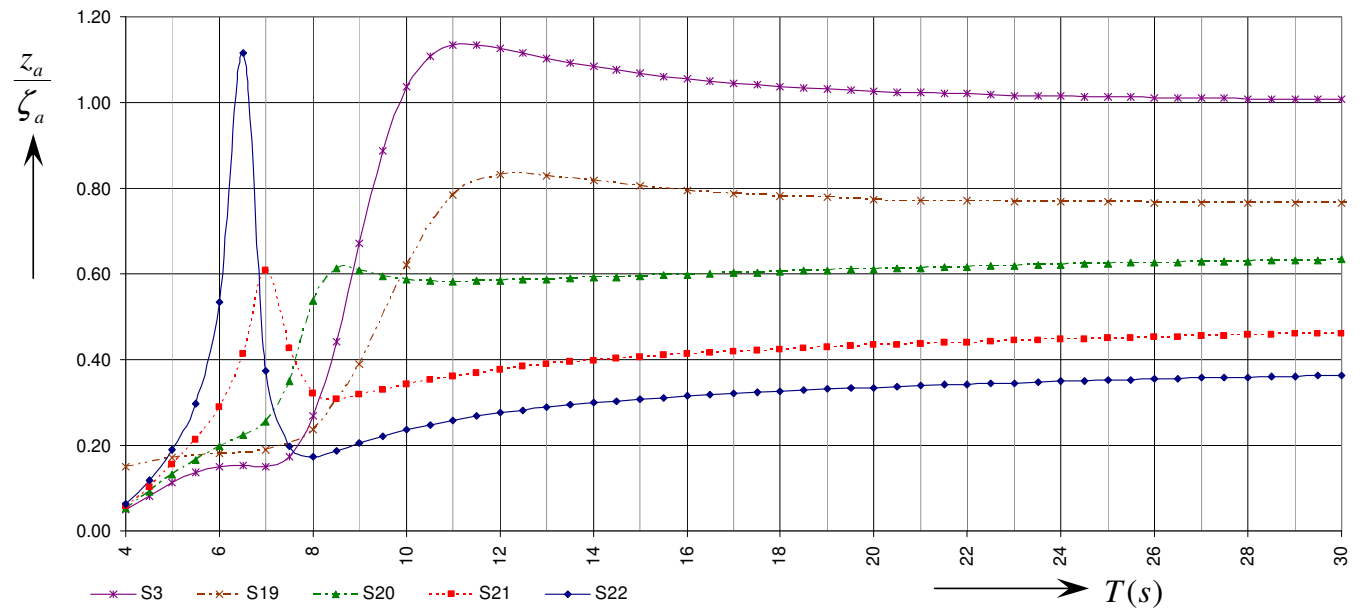

Figure C-15: Heave reaction for structures S3 and S19-S22. 


\section{C.III Wave transmission}

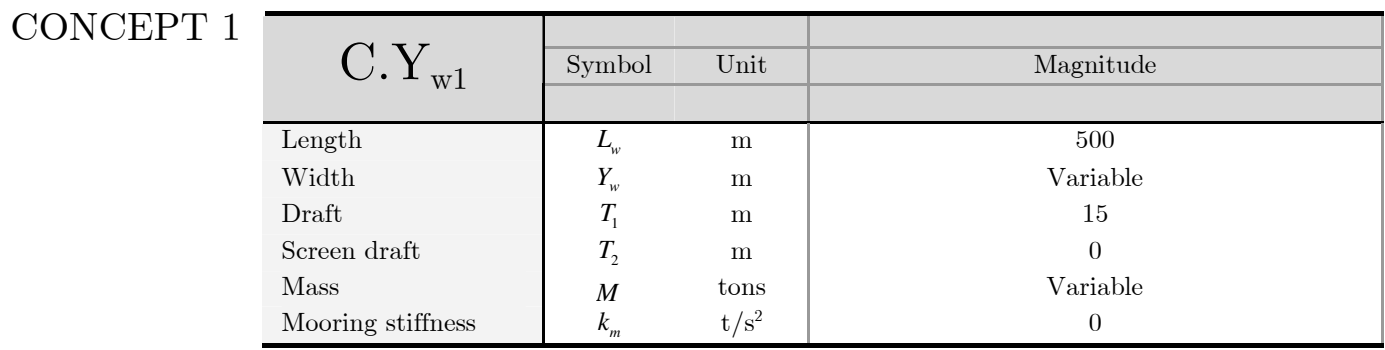

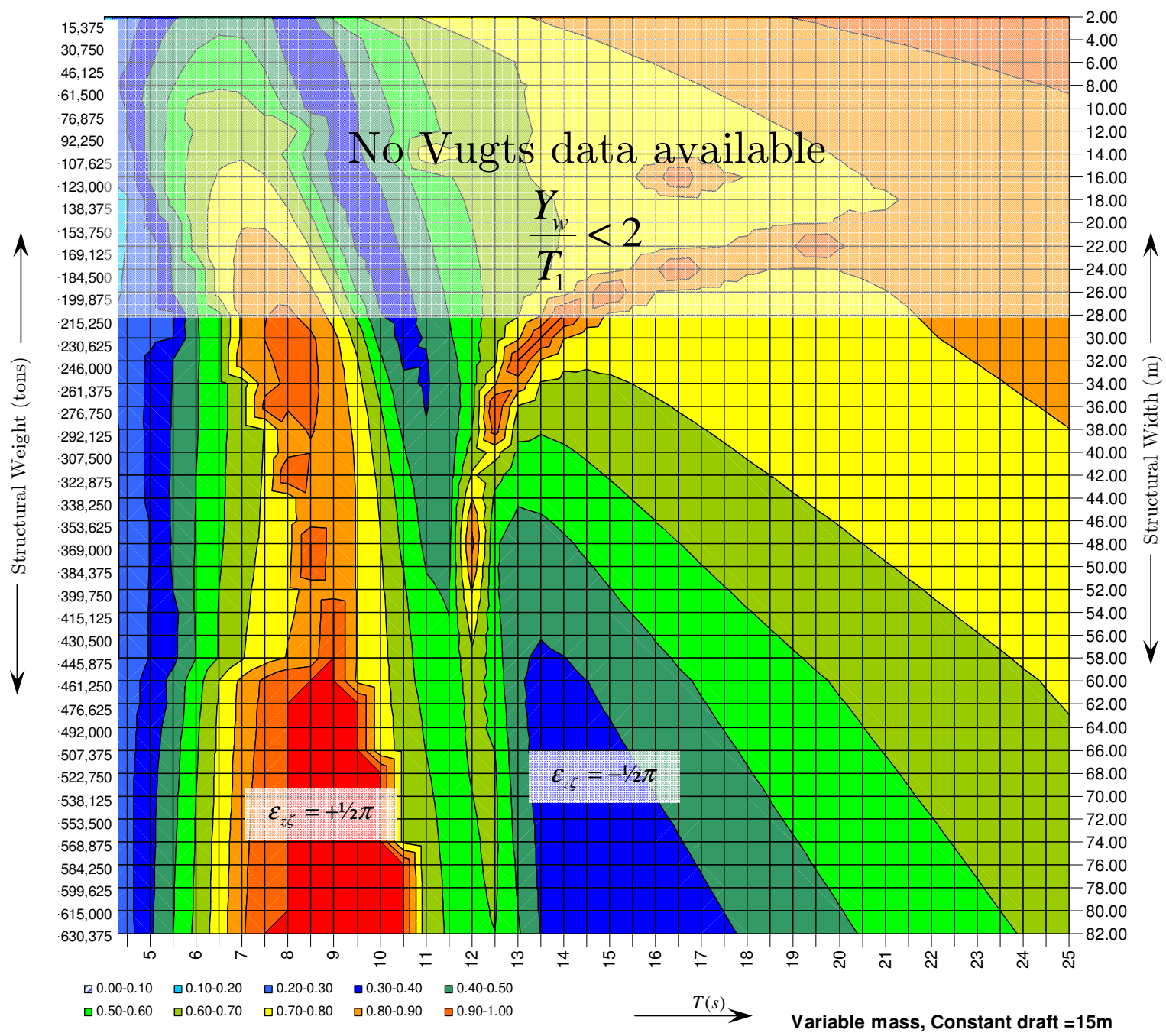

Figure C-16: Wave transmission RAO for a structure with a variable width coupled to a variable weight and constant draft.

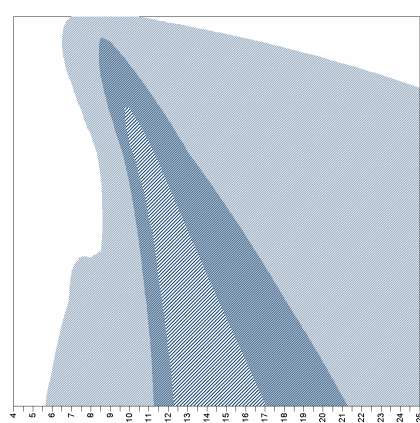

Heave

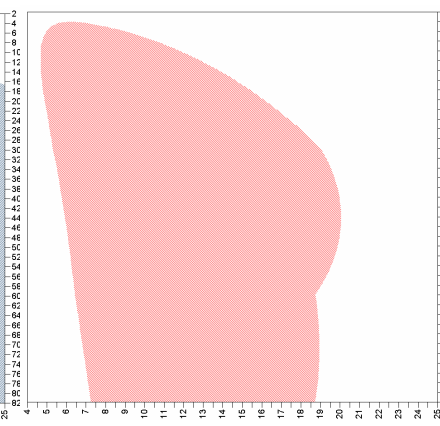

Sway

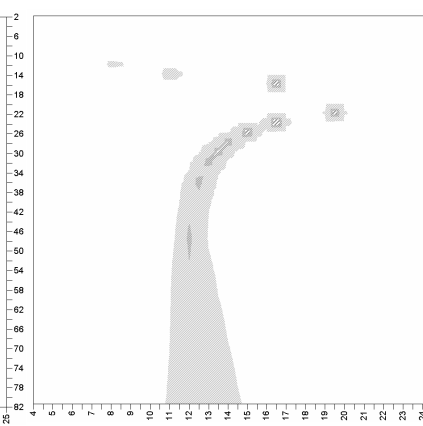

Roll

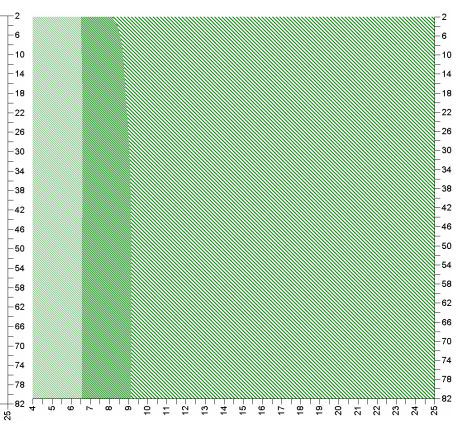

Underflow 


\begin{tabular}{|c|c|c|c|c|}
\hline \multirow[t]{7}{*}{ CONCEPT 2} & C. $\mathrm{Y}_{\mathrm{w} 2}$ & Symbol & Unit & Magnitude \\
\hline & Length & $L_{w}$ & $\mathrm{~m}$ & 500 \\
\hline & Width & $Y_{w}$ & $\mathrm{~m}$ & Variable \\
\hline & Draft & $T_{1}$ & $\mathrm{~m}$ & Variable \\
\hline & Screen draft & $T_{2}$ & $\mathrm{~m}$ & 0 \\
\hline & Mass & $M$ & tons & 123,000 \\
\hline & Mooring stiffness & $k_{m}$ & $t / s^{2}$ & 0 \\
\hline
\end{tabular}

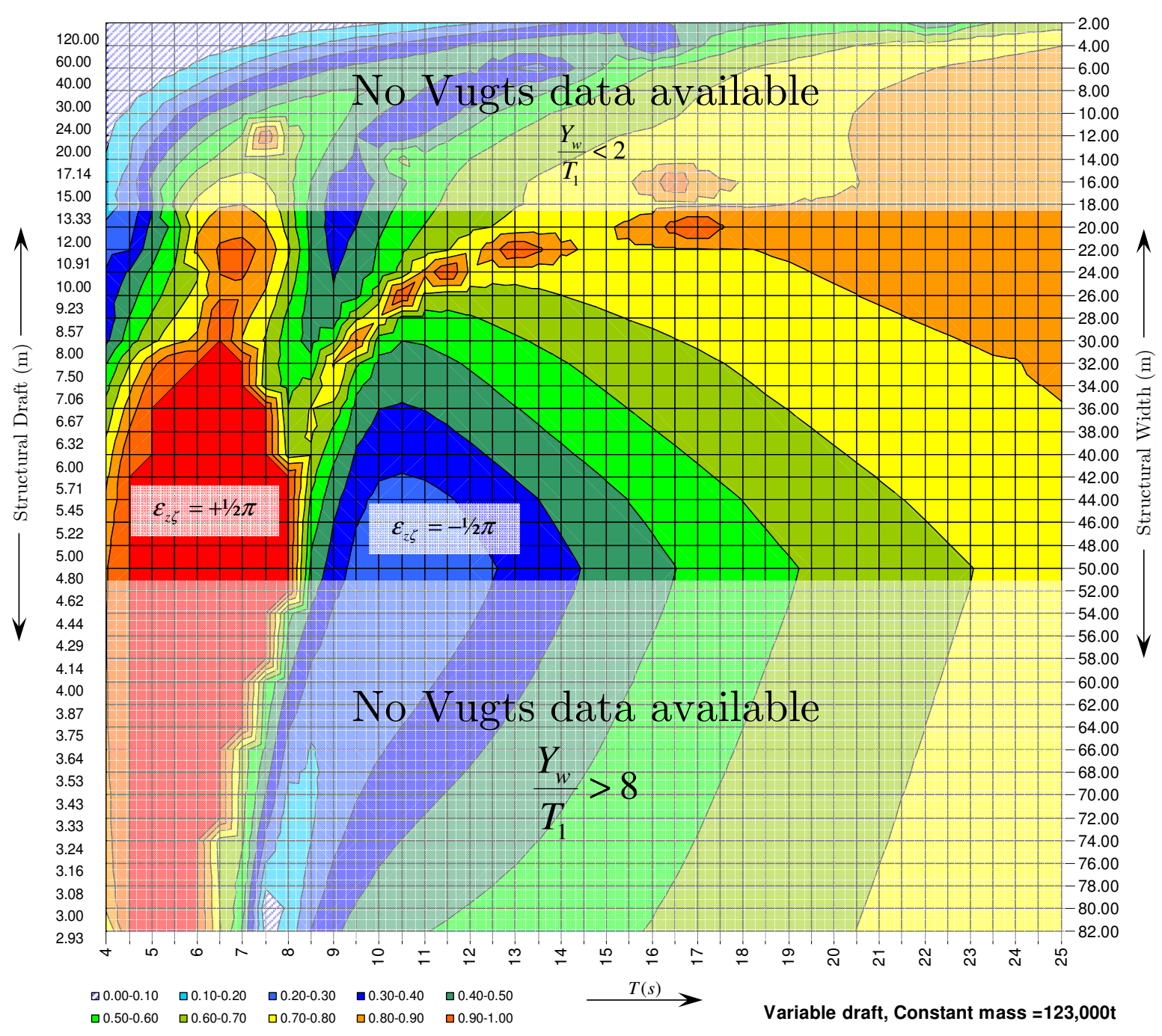

Figure C-17: Wave transmission RAO for a structure with a variable width coupled to a variable draft and a constant weight.

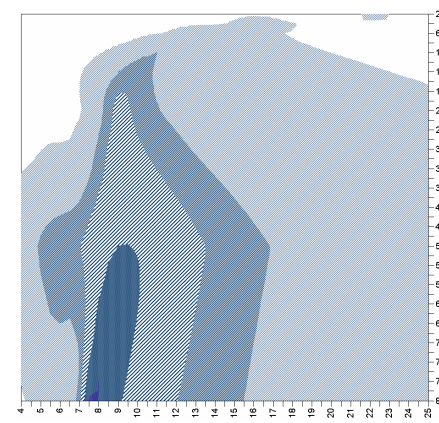

Heave

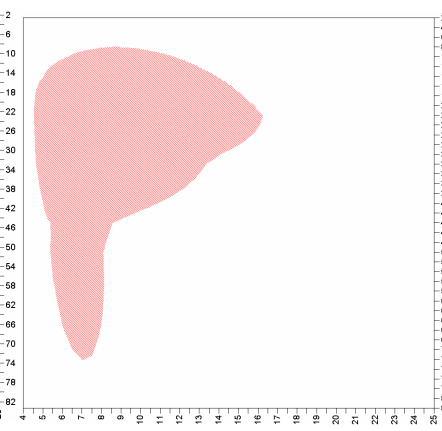

Sway

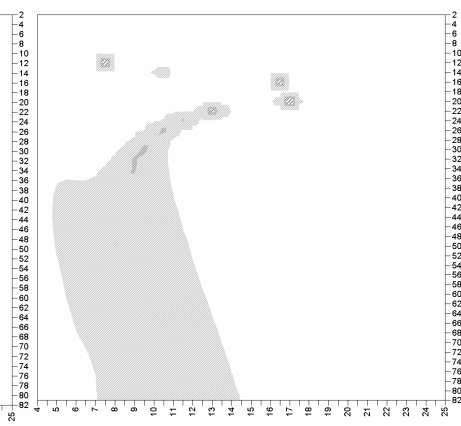

Roll

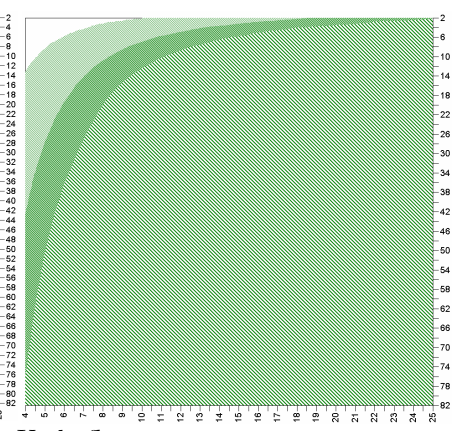

Underflow 


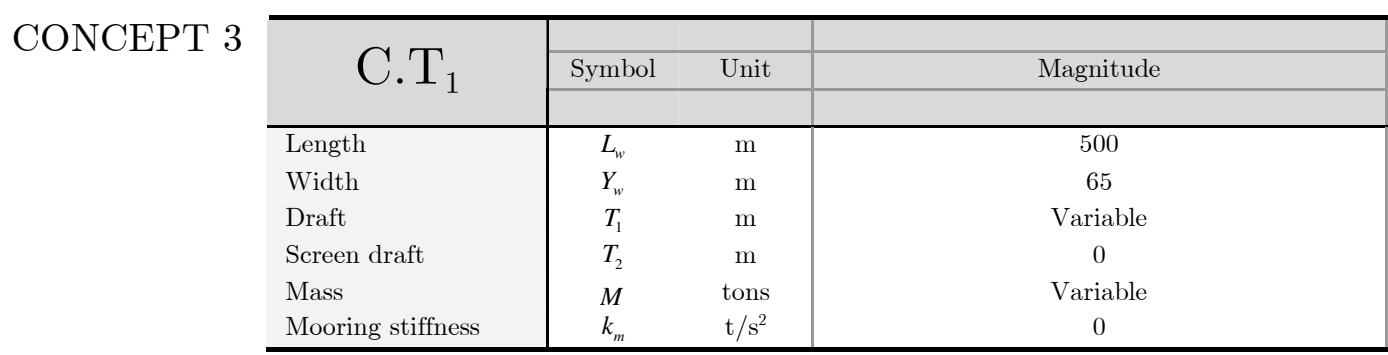

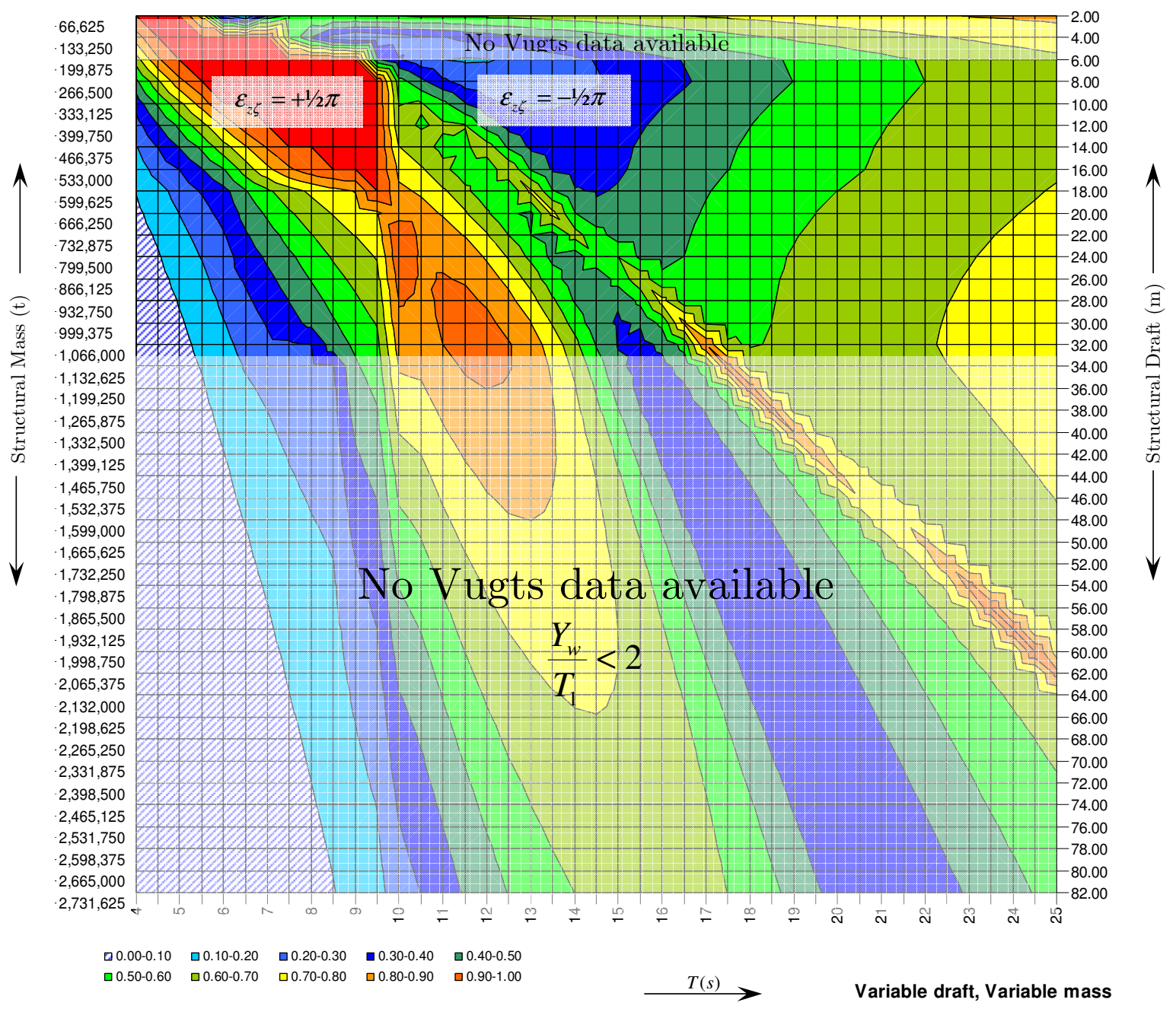

Figure C-18: Wave transmission RAO for a structure with a variable draft and thus a varying weight.

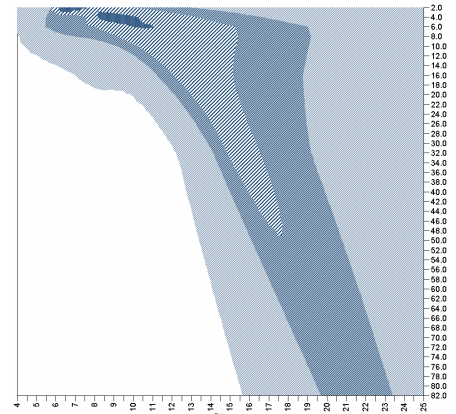

Heave

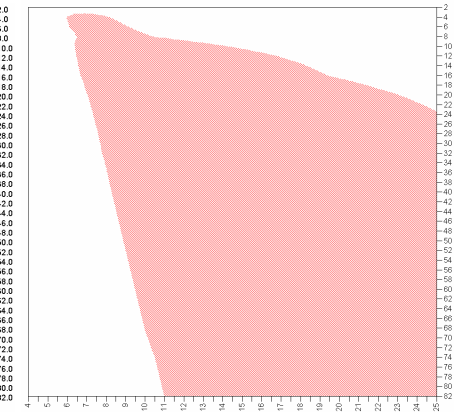

Sway

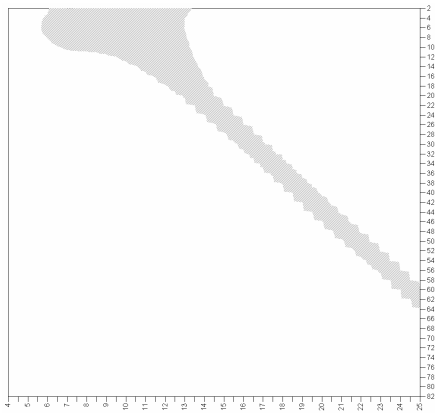

Roll

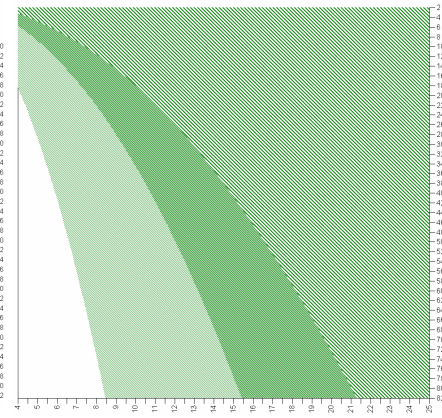

Underflow 


\begin{tabular}{|c|c|c|c|c|}
\hline \multirow{2}{*}{ CONCEPT 4} & \\
\hline & C. $\mathrm{Y}_{\mathrm{w}} \cdot \mathrm{T}_{1}$ & Symbol & Unit & Magnitude \\
\hline & Length & $L_{w}$ & $\mathrm{~m}$ & 500 \\
\hline & Width & $Y_{w}$ & $\mathrm{~m}$ & Variable \\
\hline & Draft & $T_{1}$ & $\mathrm{~m}$ & Variable \\
\hline & Screen draft & $T_{2}$ & $\mathrm{~m}$ & 0 \\
\hline & Mass & $M$ & tons & Variable \\
\hline & Mooring stiffness & $k_{m}$ & $t / s^{2}$ & 0 \\
\hline
\end{tabular}

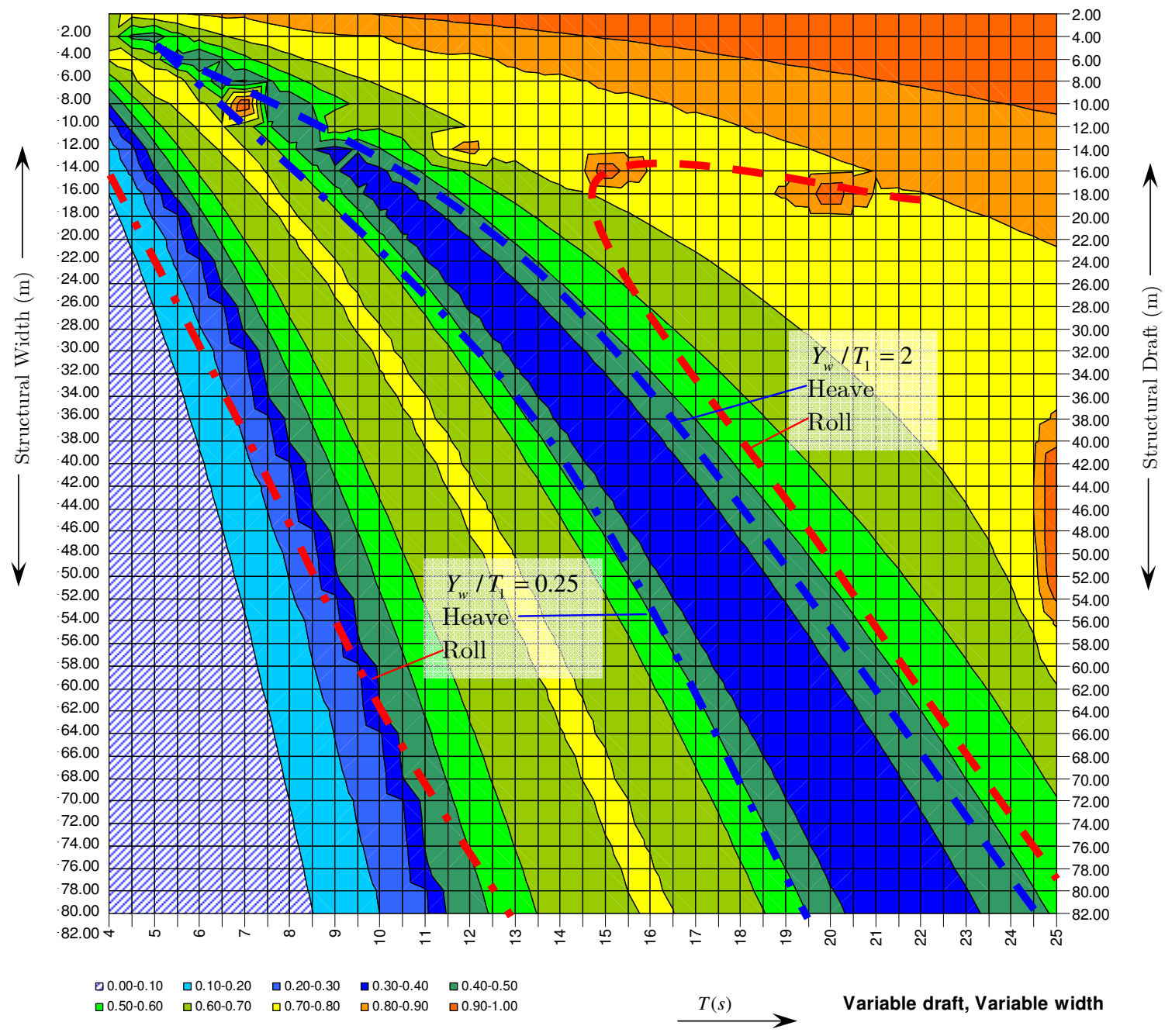

Figure C-19: Wave transmission RAO for a structure with an individual variable draft and thus a varying width.

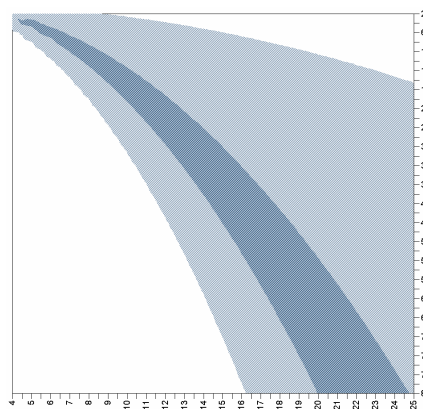

Heave

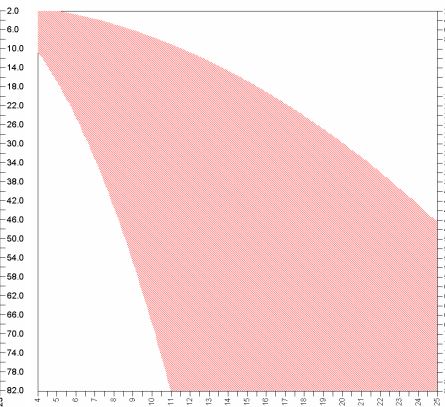

Sway

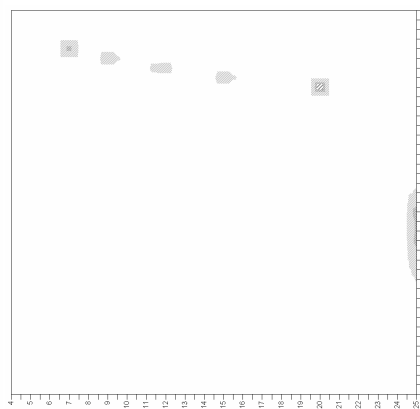

Roll

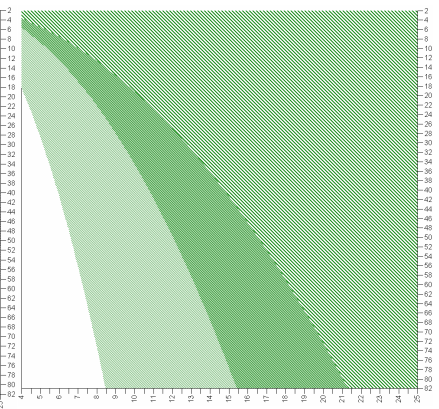

Underflow 


\begin{tabular}{|ll|cc|c|}
\cline { 2 - 4 } CONCEPT 5 & \multicolumn{1}{c|}{$\mathrm{C} . \mathrm{T}_{2}$} & Symbol & Unit & Magnitude \\
\cline { 2 - 5 } & \multicolumn{2}{c|}{} & & 500 \\
\cline { 2 - 5 } & Length & $L_{w}$ & $\mathrm{~m}$ & 45 \\
& Width & $Y_{w}$ & $\mathrm{~m}$ & 15 \\
& Draft & $T_{1}$ & $\mathrm{~m}$ & Variable \\
& Screen draft & $T_{2}$ & $\mathrm{~m}$ & 345,938 \\
& Mass & $M$ & tons & 0 \\
\hline
\end{tabular}

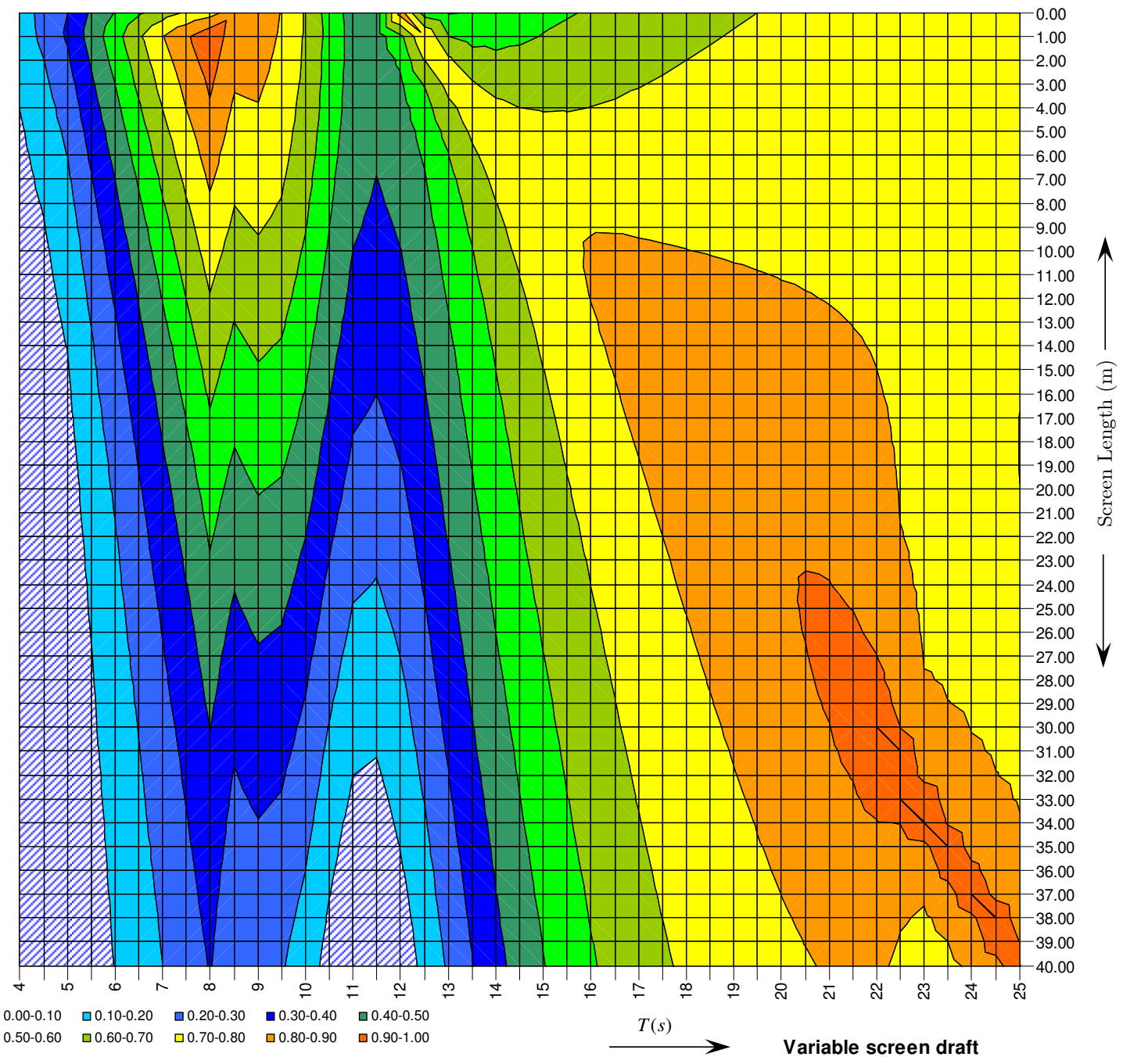

Figure C-20: Wave transmission RAO for a structure with a variable screen length.

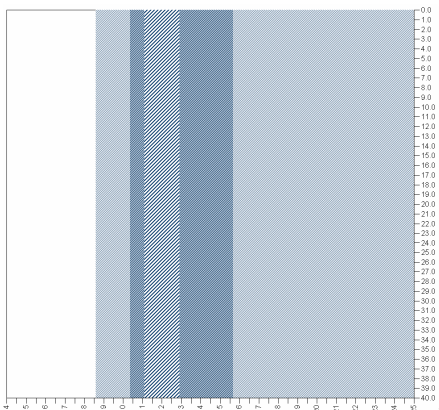

Heave

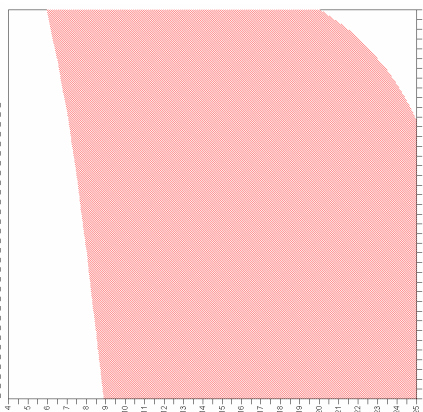

Sway

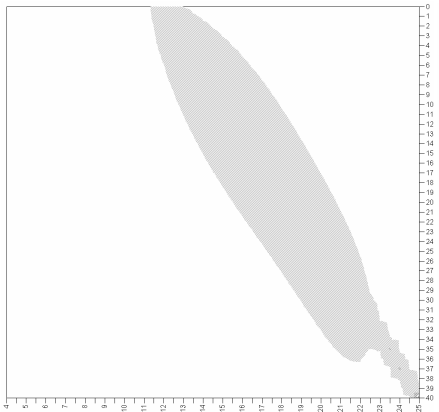

Roll

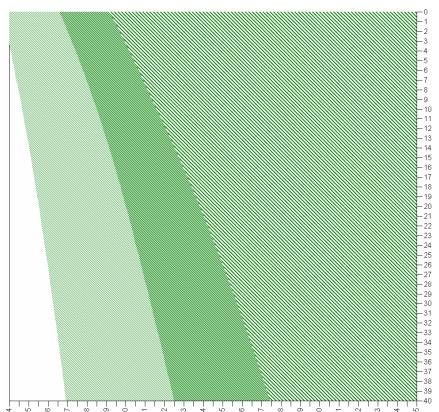

Underflow 


\begin{tabular}{|c|c|c|c|c|}
\hline \multirow[t]{2}{*}{ CONCEPT 6} & \multirow{2}{*}{$\mathrm{C} \cdot \mathrm{k}_{\mathrm{m}}$} & Symbol & Unit & Magnitude \\
\hline & & & & \\
\hline & Length & $L_{w}$ & $\mathrm{~m}$ & 500 \\
\hline & Width & $Y_{w}^{w}$ & $\mathrm{~m}$ & 45 \\
\hline & Draft & $T_{1}$ & $\mathrm{~m}$ & 15 \\
\hline & Screen draft & $T_{2}$ & $\mathrm{~m}$ & 0 \\
\hline & Mass & $M$ & tons & 345,938 \\
\hline & Mooring stiffness & $k_{m}$ & $\mathrm{t} / \mathrm{s}^{2}$ & Variable \\
\hline
\end{tabular}

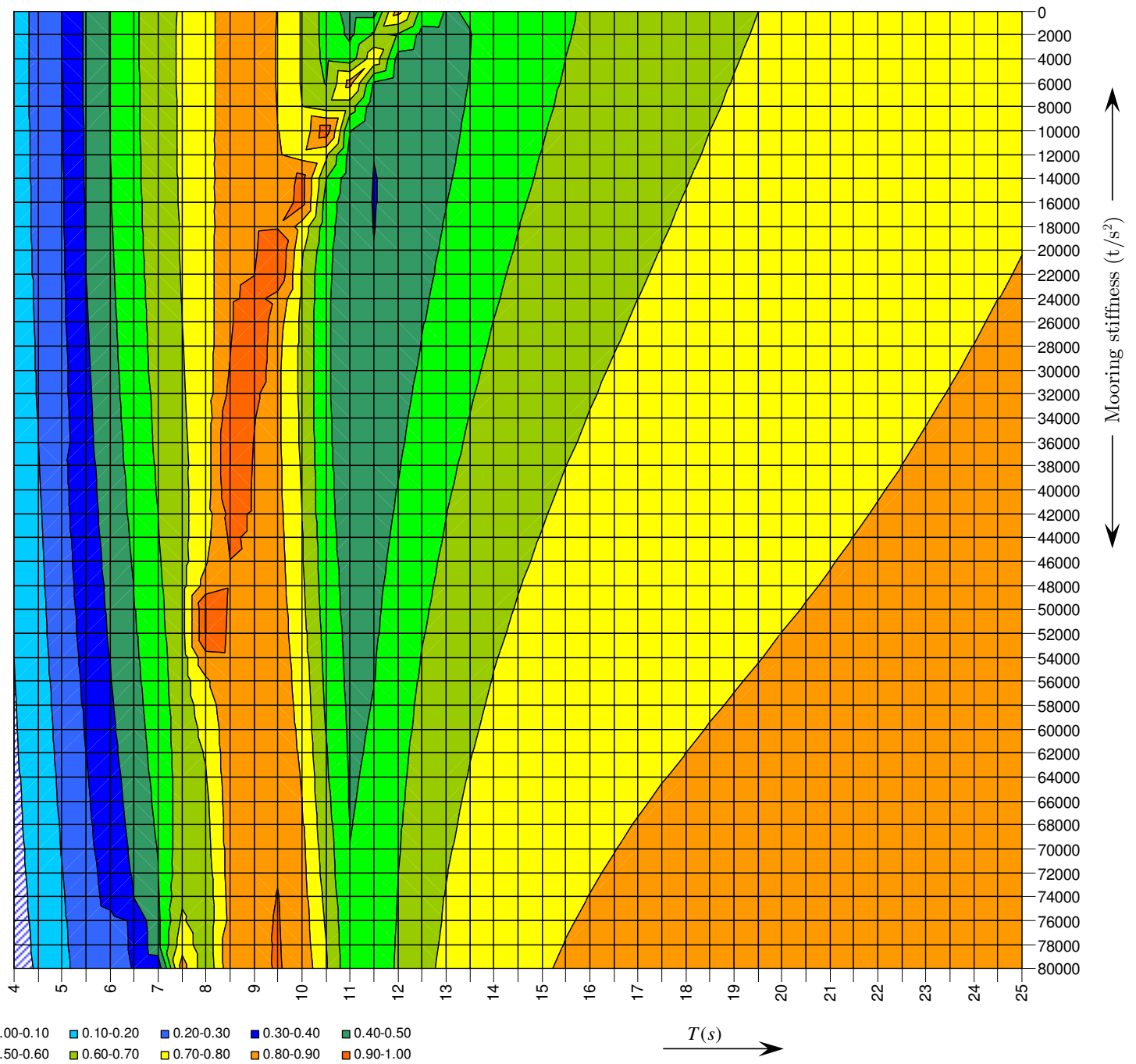

$\square 0.50-0.60 \quad \square 0.60-0.70 \quad \square 0.70-0.80 \quad \square 0.80-0.90 \quad \square 0.90-1.00$

Figure C-21: Wave transmission RAO for a structure with a variable mooring stiffness.

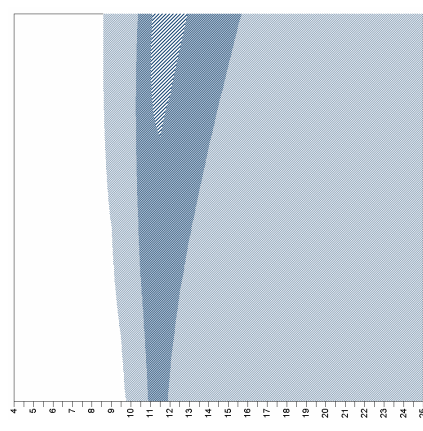

Heave

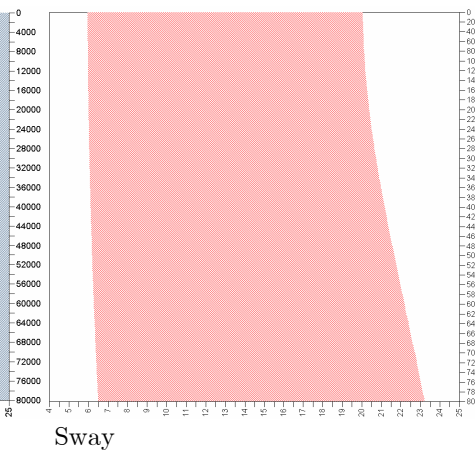

Sway

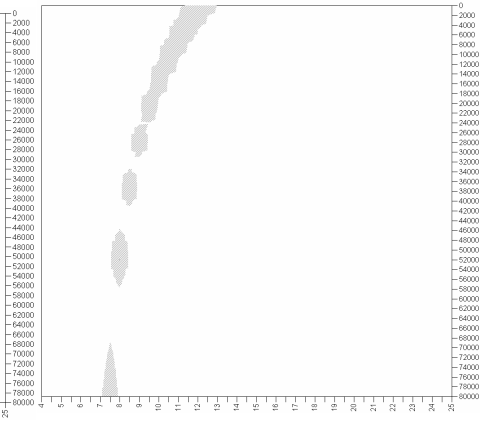

Roll

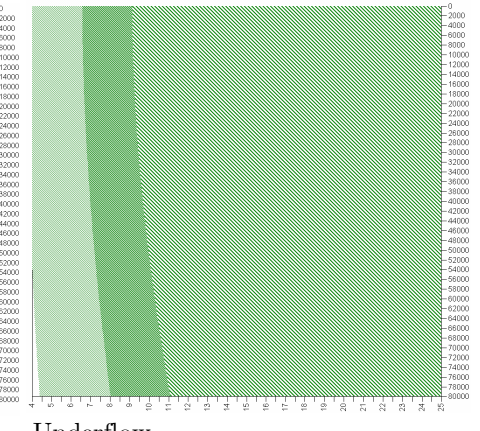

Underflow 


\section{OPTIMAL FLOATING BREAKWATER DESIGN}

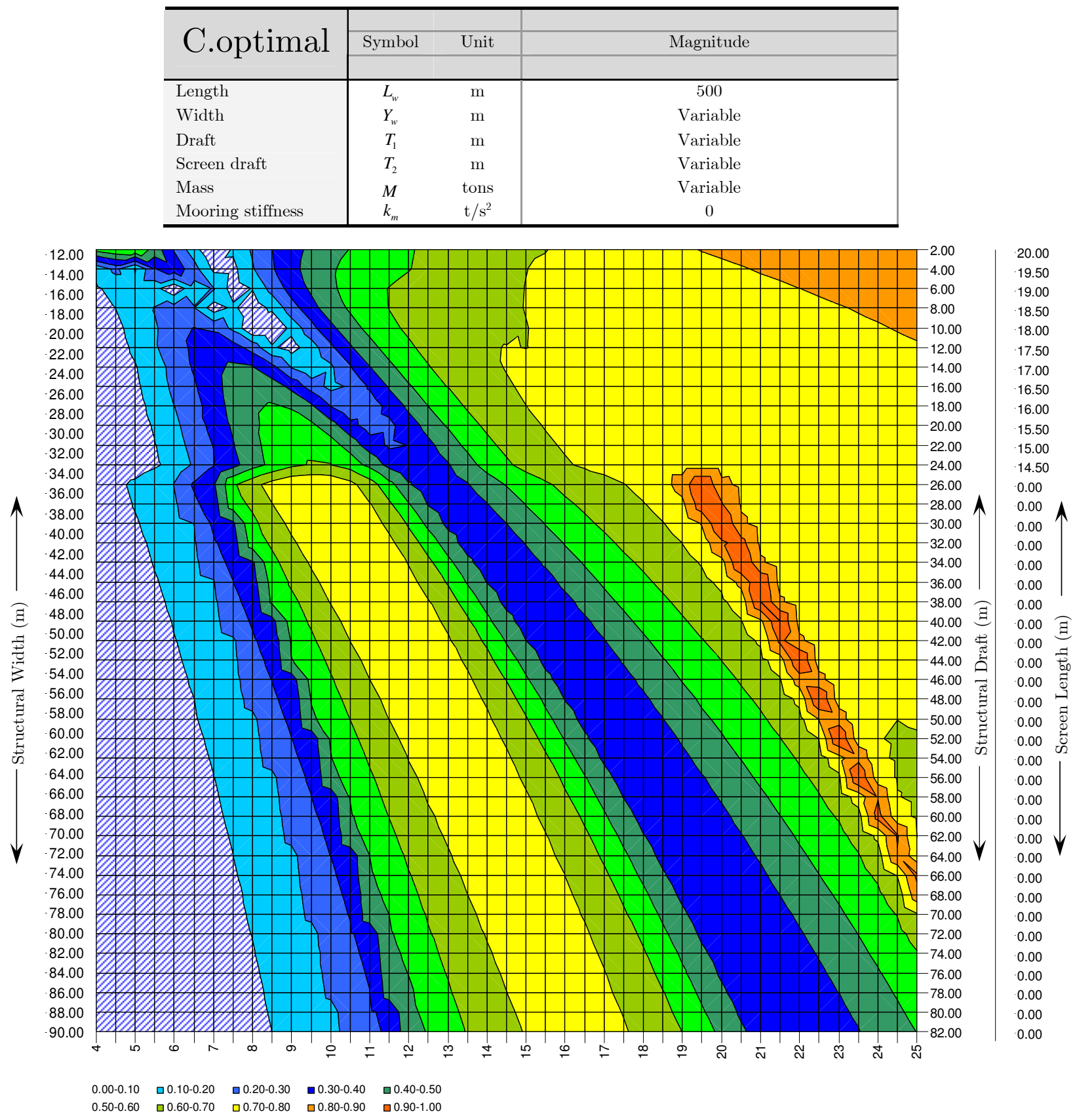

Figure C-22: Optimally performing floating breakwater.

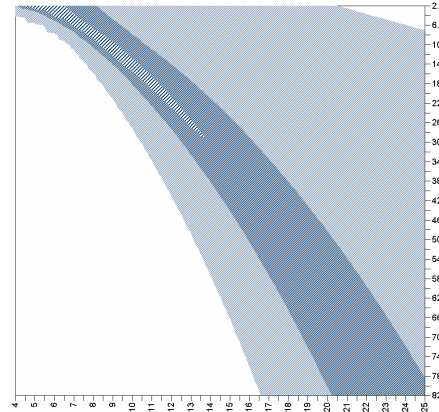

Heave

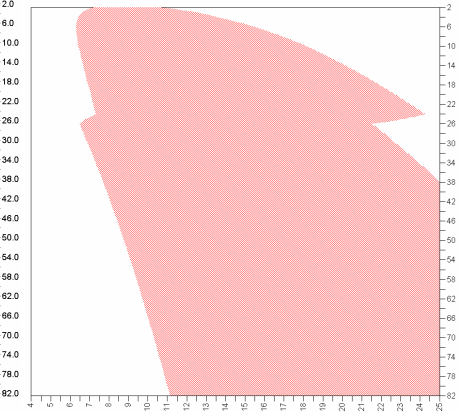

Sway

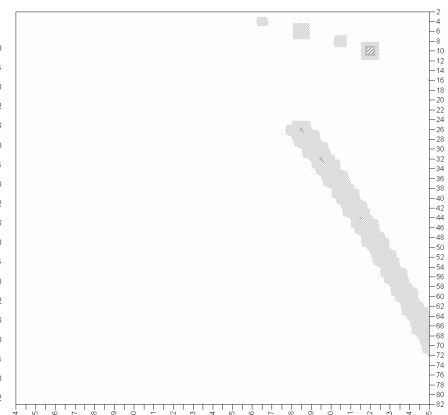

Roll

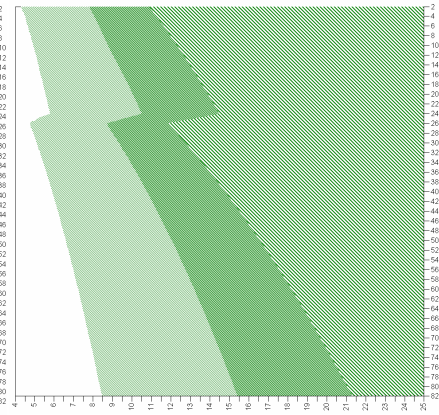

Underflow 


\section{Appendix D}

\section{Sensitivity Analysis}
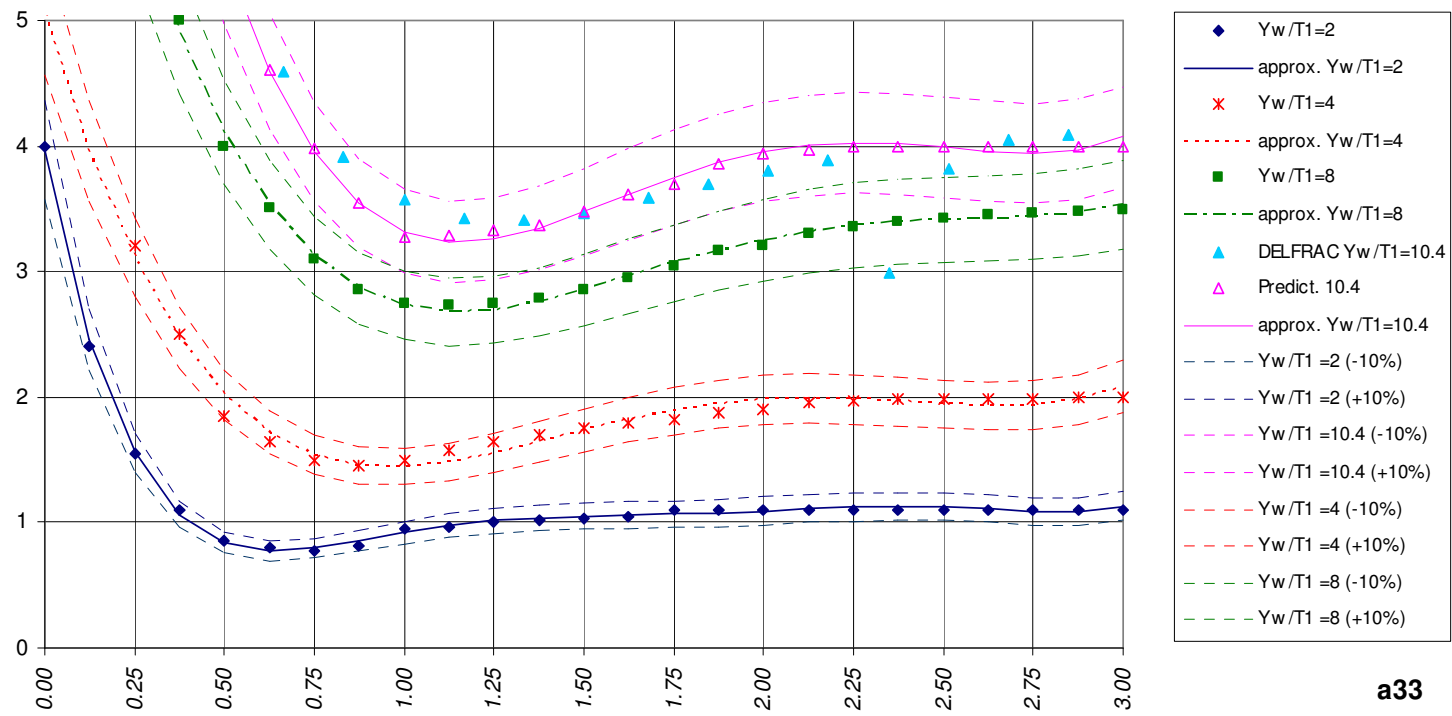

Figure D-1: Heave added mass with $\pm 10 \%$ deviation.
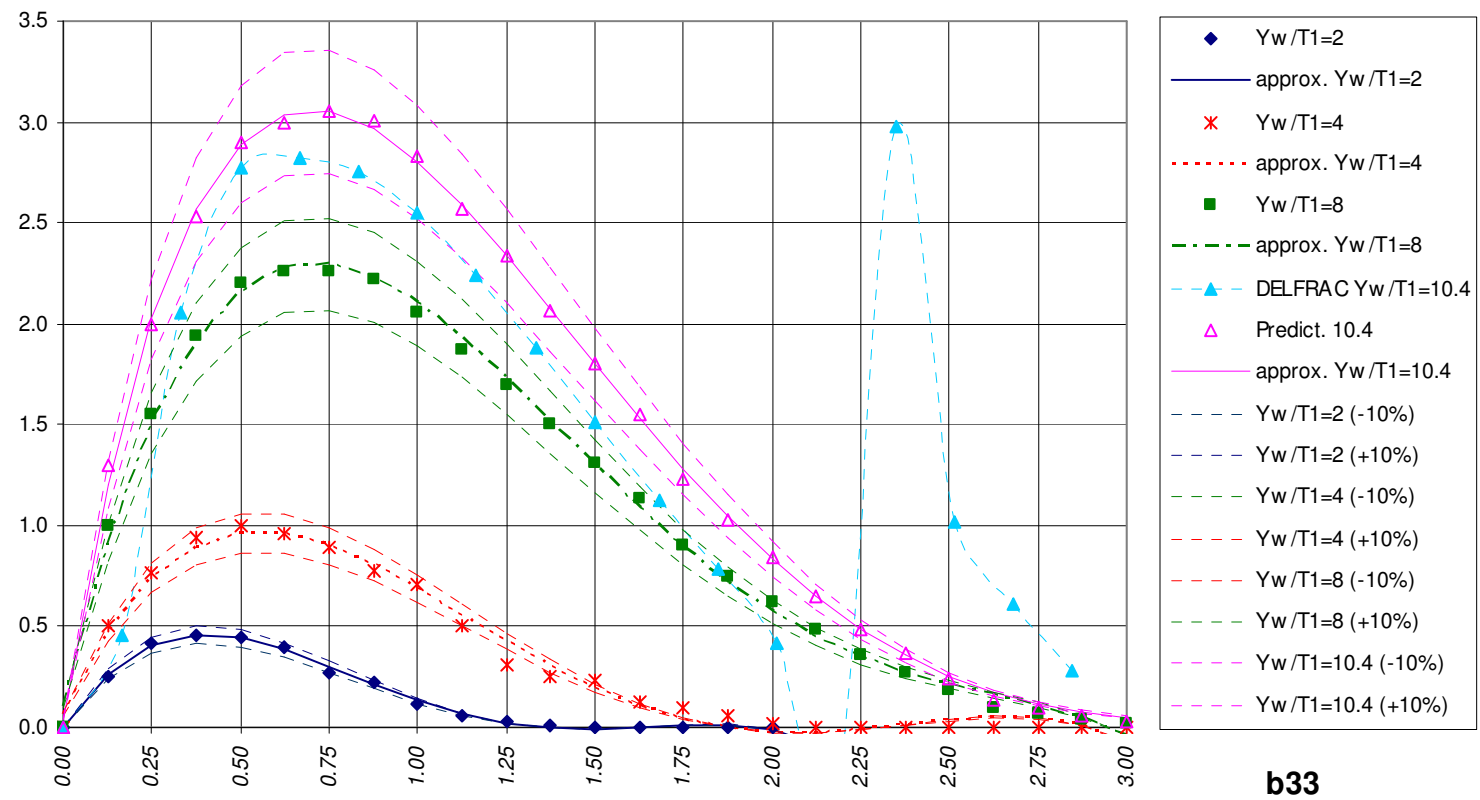

Figure D-2: Heave damping with $\pm 10 \%$ deviation. 


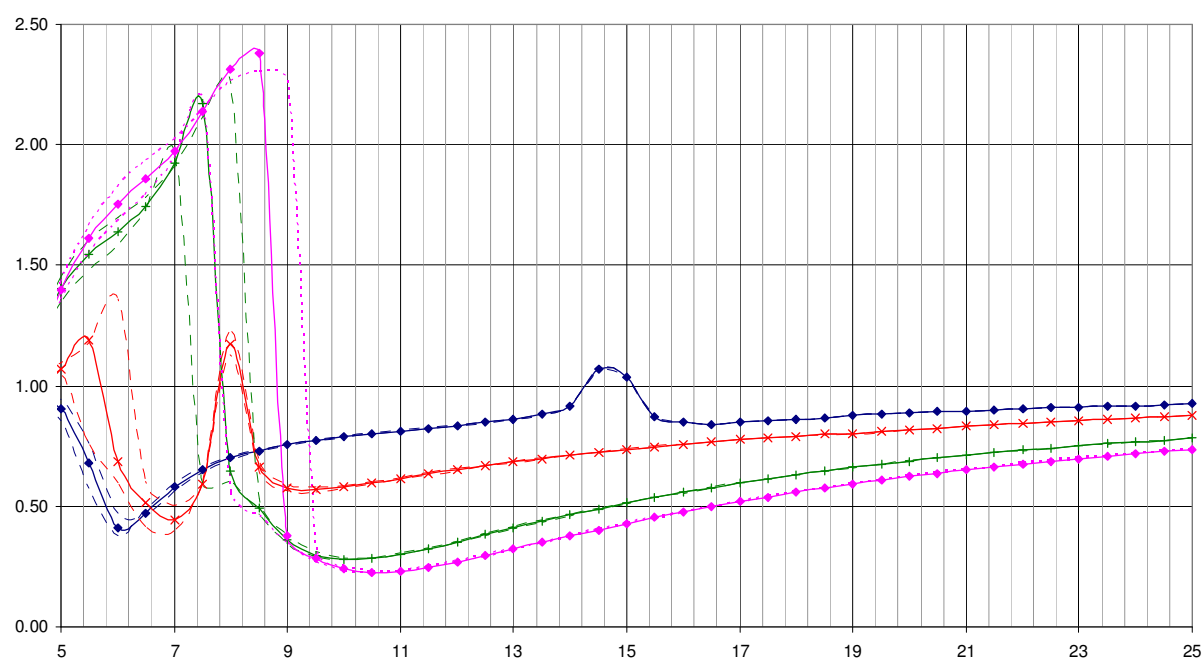

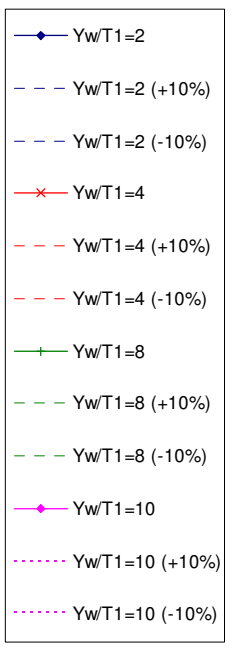

Figure D-3: Sensitivity analysis of the heave hydrodynamic added mass coefficient.
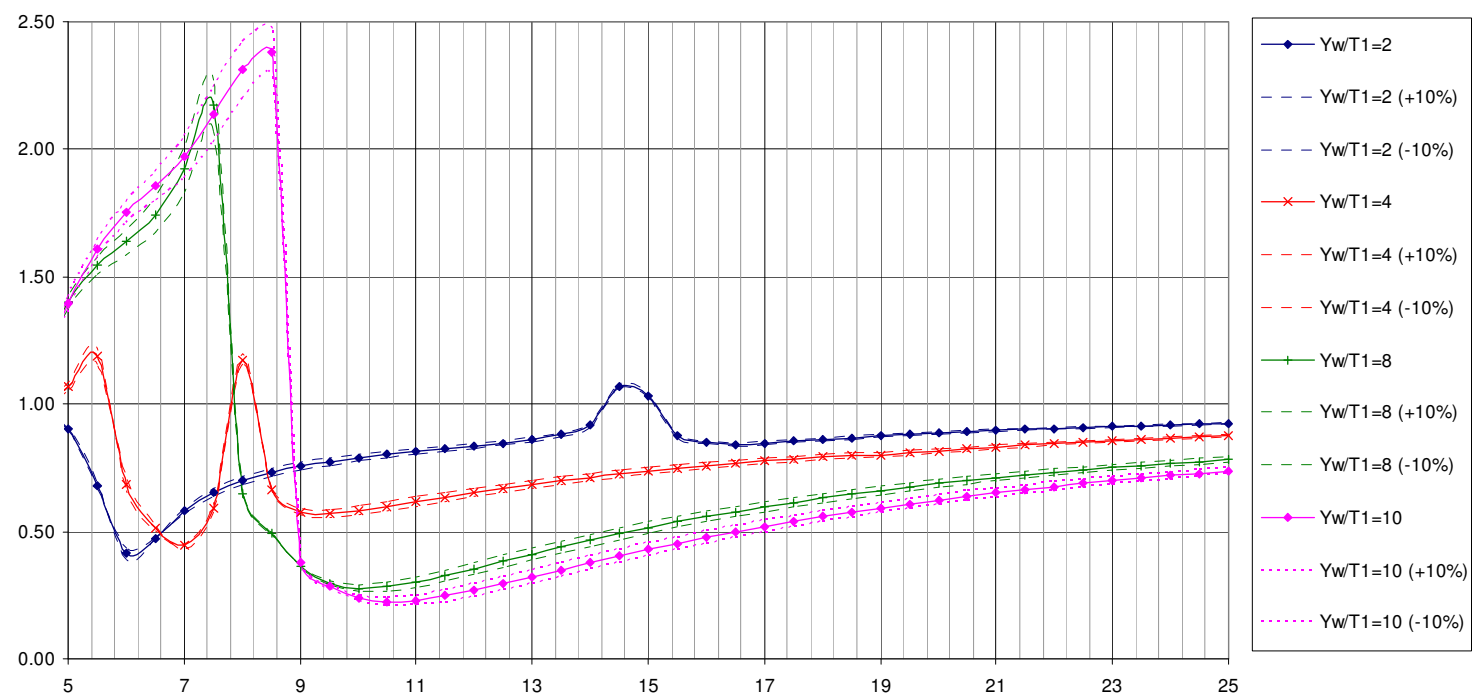

Figure D-4: Sensitivity analysis of the heave hydrodynamic damping coefficient. 


\section{Appendix E}

\section{Wave statistics Durban}

\begin{tabular}{|c|c|c|c|c|c|c|c|c|c|c|c|c|c|c|c|c|c|c|c|}
\hline & lower & 3 & 4 & 5 & 6 & 7 & 8 & 9 & 10 & 11 & 12 & 13 & 14 & 15 & 16 & 17 & 18 & & cumulative \\
\hline lower & upper & 4 & 5 & 6 & 7 & 8 & 9 & 10 & 11 & 12 & 13 & 14 & 15 & 16 & 17 & 18 & 19 & total & \\
\hline 0.0 & 1.0 & 1 & 10 & 32 & 38 & 21 & 6 & 2 & 0 & 0 & 0 & 0 & 0 & 0 & 0 & 0 & 0 & 110 & 110 \\
\hline 1.0 & 2.0 & 0 & 4 & 37 & 96 & 101 & 57 & 21 & 6 & 1 & 0 & 0 & 0 & 0 & 0 & 0 & 0 & 323 & 432 \\
\hline 2.0 & 3.0 & 0 & 1 & 12 & 51 & 84 & 71 & 36 & 13 & 4 & 1 & 0 & 0 & 0 & 0 & 0 & 0 & 273 & 705 \\
\hline 3.0 & 4.0 & 0 & 0 & 3 & 18 & 40 & 45 & 30 & 13 & 4 & 1 & 0 & 0 & 0 & 0 & 0 & 0 & 155 & 860 \\
\hline 4.0 & 5.0 & 0 & 0 & 1 & 6 & 16 & 22 & 17 & 9 & 4 & 1 & 0 & 0 & 0 & 0 & 0 & 0 & 76 & 936 \\
\hline 5.0 & 6.0 & 0 & 0 & 0 & 2 & 6 & 9 & 9 & 6 & 2 & 1 & 0 & 0 & 0 & 0 & 0 & 0 & 35 & 971 \\
\hline 6.0 & 7.0 & 0 & 0 & 0 & 1 & 2 & 4 & 4 & 3 & 2 & 0 & 0 & 0 & 0 & 0 & 0 & 0 & 15 & 986 \\
\hline 7.0 & 8.0 & 0 & 0 & 0 & 0 & 1 & 2 & 2 & 2 & 1 & 0 & 0 & 0 & 0 & 0 & 0 & 0 & 7 & 993 \\
\hline 8.0 & 9.0 & 0 & 0 & 0 & 0 & 0 & 1 & 1 & 1 & 0 & 0 & 0 & 0 & 0 & 0 & 0 & 0 & 3 & 995 \\
\hline 9.0 & 10.0 & 0 & 0 & 0 & 0 & 0 & 0 & 0 & 0 & 0 & 0 & 0 & 0 & 0 & 0 & 0 & 0 & 1 & 996 \\
\hline 10.0 & 11.0 & 0 & 0 & 0 & 0 & 0 & 0 & 0 & 0 & 0 & 0 & 0 & 0 & 0 & 0 & 0 & 0 & 0 & 997 \\
\hline 11.0 & 12.0 & 0 & 0 & 0 & 0 & 0 & 0 & 0 & 0 & 0 & 0 & 0 & 0 & 0 & 0 & 0 & 0 & 0 & 997 \\
\hline 12.0 & 13.0 & 0 & 0 & 0 & 0 & 0 & 0 & 0 & 0 & 0 & 0 & 0 & 0 & 0 & 0 & 0 & 0 & 0 & 997 \\
\hline 13.0 & 14.0 & 0 & 0 & 0 & 0 & 0 & 0 & 0 & 0 & 0 & 0 & 0 & 0 & 0 & 0 & 0 & 0 & 0 & 997 \\
\hline 14.0 & 15.0 & 0 & 0 & 0 & 0 & 0 & 0 & 0 & 0 & 0 & 0 & 0 & 0 & 0 & 0 & 0 & 0 & 0 & 997 \\
\hline 15.0 & 16.0 & 0 & 0 & 0 & 0 & 0 & 0 & 0 & 0 & 0 & 0 & 0 & 0 & 0 & 0 & 0 & 0 & 0 & 997 \\
\hline 16.0 & 17.0 & 0 & 0 & 0 & 0 & 0 & 0 & 0 & 0 & 0 & 0 & 0 & 0 & 0 & 0 & 0 & 0 & 0 & 997 \\
\hline 17.0 & 18.0 & 0 & 0 & 0 & 0 & 0 & 0 & 0 & 0 & 0 & 0 & 0 & 0 & 0 & 0 & 0 & 0 & 0 & 997 \\
\hline 18.0 & 19.0 & 0 & 0 & 0 & 0 & 0 & 0 & 0 & 0 & 0 & 0 & 0 & 0 & 0 & 0 & 0 & 0 & 0 & 997 \\
\hline 19.0 & 20.0 & 0 & 0 & 0 & 0 & 0 & 0 & 0 & 0 & 0 & 0 & 0 & 0 & 0 & 0 & 0 & 0 & 0 & 997 \\
\hline 20.0 & 21.0 & 0 & 0 & 0 & 0 & 0 & 0 & 0 & 0 & 0 & 0 & 0 & 0 & 0 & 0 & 0 & 0 & 0 & 997 \\
\hline 21.0 & 22.0 & 0 & 0 & 0 & 0 & 0 & 0 & 0 & 0 & 0 & 0 & 0 & 0 & 0 & 0 & 0 & 0 & 0 & 997 \\
\hline \multirow{2}{*}{\multicolumn{2}{|c|}{ total }} & 1 & 15 & 85 & 211 & 270 & 217 & 121 & 52 & 18 & 5 & 1 & 0 & 0 & 0 & 0 & 0 & 997 & \\
\hline & & 1 & 16 & 101 & 312 & 582 & 799 & 921 & 973 & 991 & 996 & 997 & 997 & 997 & 997 & 997 & 997 & & \\
\hline
\end{tabular}

Table E-1: Annual wave statistics for Area 90 of the Global Wave Statistics.

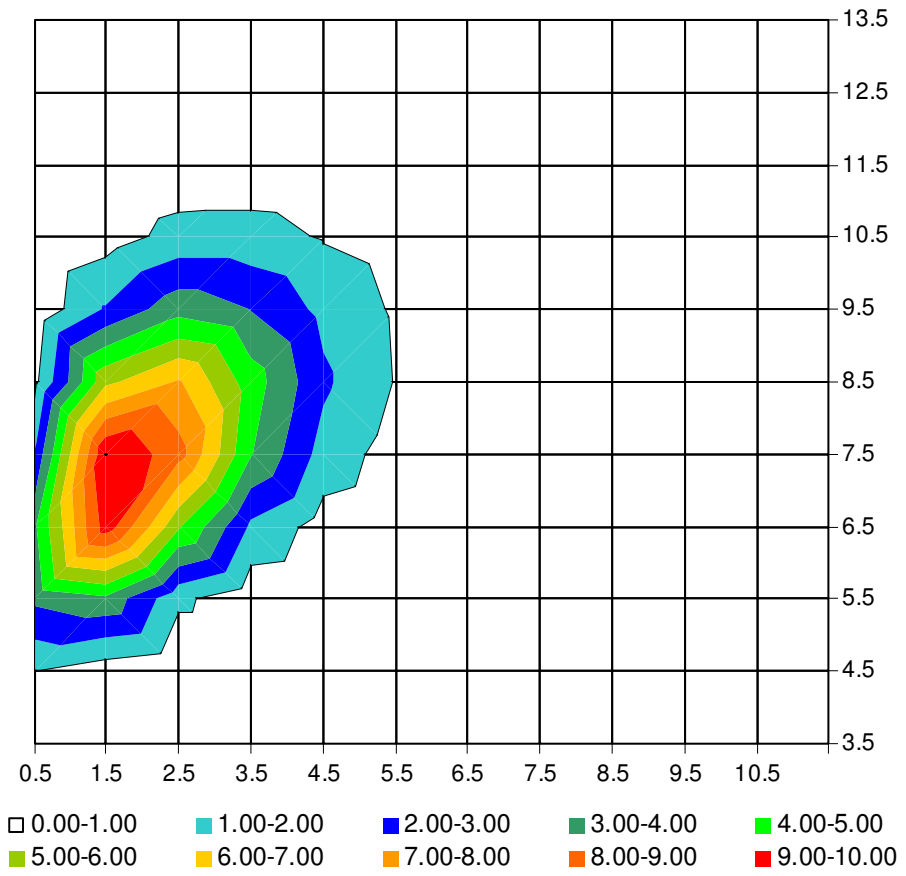

Figure E-1: Graphical representation of table E-1 [\%]. 